\title{
Goodput Modelling and Optimisation of Channel Assignment For Planning IEEE 802.11 Wireless Backhaul Networks
}

\author{
by
}

Ying Qu

\author{
A thesis \\ submitted to the Victoria University of Wellington \\ in fulfilment of the \\ requirements for the degree of \\ Doctor of Philosophy \\ in Engineering.
}

Victoria University of Wellington

2018 



\begin{abstract}
IEEE 802.11 Wireless backhaul networks (WBNs) provide scalable and costeffective solutions for interconnecting small-cell networks and backbone networks or Internet. With newer and farther reaching applications being developed in IEEE 802.11 WBNs, such as smart grids and intelligent transportation systems, users expect high goodput and better fairness. However, some performance issues in IEEE 802.11 protocols such as border effect, exposed nodes and hidden nodes are exacerbated as network densification occurs, leading to goodput degradation and severe unfairness such as flow starvation (extreme low goodput). These issues may cause an IEEE 802.11 WBN to form a bottleneck and impact the overall network performance. Therefore, in-depth study is required in order to improve the IEEE 802.11 WBN planning to achieve better goodput and fairness.

This research aims to improve IEEE 802.11 WBN planning through goodput modelling and optimising channel assignment. A novel simple goodput distribution model is proposed to predict goodput and fairness in IEEE 802.11 WBNs. Simulation results show that the proposed goodput model accurately predicts goodput with consideration of carrier sensing effect and traffic demands. Based on this goodput model, a new interference model is proposed to more realistically reflect both local and global interference in IEEE 802.11 WBNs. With the proposed interference model, two anti-starvation channel assignments have been developed to prevent flow starvation. Simulation validations show that the new anti-starvation channel assignments effectively prevent flow starvation and improve network fairness in IEEE 802.11 WBNs.
\end{abstract}

This research also optimises channel assignment to achieve desired 
fairness and goodput. A multi-objective optimisation problem is formulated and a new fitness function is designed to evaluate a channel allocation with accurate prediction of goodput and fairness. Utilising the new fitness function, two multi-objective channel assignments have been developed to achieve both fairness and goodput. Compared with existing channel assignments through simulation, the proposed multi-objective channel assignments provide a set of feasible solutions that meet desired fairness and goodput.

This research helps network planners or service providers to improve the IEEE 802.11 WBN planning from predicting network performance to optimising goodput and fairness. The proposed goodput model, interference model, and fitness function are also useful for node placement, and optimising routing and scheduling in IEEE 802.11 WBNs. 


\section{Acknowledgments}

I would like to express my very great appreciation to my supervisors, Dr Bryan Ng and A/Prof Peter Andreae, for their guidance, encouragement, and support during the course of my PhD study. I am grateful for the Victoria Doctoral Scholarship for the financial support during my PhD study.

I would like to thank Dr Xiaodan Gao for her guidance in critical thinking and writing. With her help, my English and writing has been improved a lot. I am very lucky to have her support on this tough journey.

I would like to offer my thanks to Roman Klapaukh for helping me from my PhD proposal to the final oral examination. Roman has always been patient to listen to my questions and help me improve my research skill and English in various ways. I appreciate all the help and support from Boxiong Tan during the last stage of my PhD. Boxiong's encouragement helps me find the courage to finish my thesis. In my heart, they are more than friends but my brothers as well.

Thank Alexander Deng, Alexandre Sawczuk da Silva, Arindam Bhakta, Bing Xue, David Harrison, Deepak Singh, Denise Culhane, Hang Yu, Karen Commons, Liang Yang, Michael Homer, Siyun Thompson, Timo Hönig and Wenlong $\mathrm{Fu}$ for their support and help in my research.

Thank my parents, my nephew Toby, and niece Tiffany who have always been offering their unconditional love and care.

Thank my examiners Dr Ian Welch, Dr Andreas Willig, and Dr Karina Mabell Gomez for the suggestions that improve the quality of my thesis and inspire me with new ideas for future work. 


\section{Contents}

1 Introduction 1

1.1 Research Goals . . . . . . . . . . . . . . . . . . . 3

1.2 Contributions . . . . . . . . . . . . . . 4

1.3 List of Publications . . . . . . . . . . . . . . . . . . 5

1.4 Organisation of Thesis $\ldots \ldots \ldots \ldots \ldots$

2 Related Work 11

2.1 Definitions of Key Terms . . . . . . . . . . . . . . . . . 11

2.2 IEEE 802.11 Wireless Backhaul Networks . . . . . . . . . . 13

2.2.1 Architecture and Application Scenarios . . . . . . . . 14

2.2.2 The Performance Issues and Network Planning . . . 16

2.3 Goodput Modelling For Planning IEEE 802.11 WBNs _. . . 18

2.3.1 Goodput Models Considering Structured Topology . 19

2.3.2 Goodput Models Considering Carrier Sensing Mechanism . . . . . . . . . . . . . . 20

2.3 .3 Goodput Models Considering Traffic Demand . . . . 22

2.3 .4 Summary of Goodput Models . . . . . . . . . . . 23

2.4 Channel Assignment For Planning IEEE 802.11 WBNs . . . . 24

2.4.1 Channel Assignment in IEEE 802.11 WBNs . . . . . . 25

2.4 .2 Traffic-unaware Channel Assignment . . . . . . . . . 26

2.4 .3 Traffic-aware Channel Assignment. . . . . . . . . . . 33

2.5 Summary . . . . . . . . . . . . . . . . . . . . . 39 
4.3.2 Benchmark For Traffic-aware Anti-starvation CA . . 114

4.3.3 Validation of Traffic-aware Anti-starvation CA Using Three Channels . . . . . . . . . . . . . . . 117

4.3.4 Validation of Traffic-aware Anti-starvation CA Using Twelve Channels . . . . . . . . . . . . . 123

4.3 .5 Discussion about Traffic-aware Anti-starvation CA . 126 4.4 Summary . . . . . . . . . . . . . . . . . . . . . . . . 127

5 Multi-objective optimisation of channel assignment 129

5.1 Fitness Function with CA Algorithm . . . . . . . . . . . . 132

$5.1 .1 \quad$ The Design of Fitness Function . . . . . . . . . . . 132

5.1.2 Validation of Traffic-unaware Fitness Function . . . . 138

5.1 .3 Validation of Traffic-aware Fitness Function . . . . 143

5.1 .4 Summary of Fitness Function . . . . . . . . . . . 145

5.2 A Traffic-unaware Multi-objective CA Algorithm . . . . . . . 146

5.2.1 The Design of Traffic-unaware Multi-objective CA . . 147

5.2.2 Validation of Traffic-unaware Multi-objective CA . 150

5.3 A Traffic-aware Multi-objective CA Algorithm . . . . . . . 154

5.3.1 The Design of Traffic-aware Multi-objective CA . . 155

5.3.2 Validation of Traffic-aware Multi-objective CA . . . 157

5.4 Summary . . . . . . . . . . . . . . . . 161

6 Conclusions 163

6.1 Contributions . . . . . . . . . . . . . . . . . . . . . . 164

6.2 Future Work . . . . . . . . . . . . . . . . . . . . . . . . . 165

6.2 .1 Goodput Modelling . . . . . . . . . . . . . . . . 166

6.2 .2 Interference Model . . . . . . . . . . . . . . . . . 166

6.2 .3 Channel Assignment with Fairness. . . . . . . . . . 166

6.2 .4 Fitness Function . . . . . . . . . . . . . . . . . 167

6.2.5 Channel Assignment with Fairness and Goodput . . 167 
Appendix A IEEE 802.11 WBN Goodput Patterns with Effective vs. \begin{tabular}{ll}
\hline Ineffective CSMA & 169
\end{tabular}

A.1 Simulation Configurations . . . . . . . . . . . . . 169

A.2 Disk-graph Model Accurately Characterizes Goodput . . . . 172

A.2.1 Saturated Traffic Demand . . . . . . . . . . . . . . 173

A.2.2 Unsaturated Traffic Demand . . . . . . . . . . . . . . 174

A.3 Disk-graph Model Fails to Characterize Goodput. . . . . . . 176

A.3.1 Saturated Traffic Demand . . . . . . . . . . . 176

A.3.2 Unsaturated Traffic Demand . . . . . . . . . . . . 177

A.4 Summary . . . . . . . . . . . . . . . . . . . . . . 179

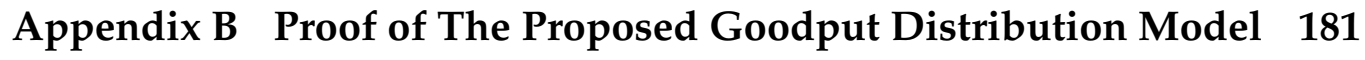

B.1 Goodput For Effective CSMA (Saturated Traffic) . . . . . . . 181

B.2 Goodput For Ineffective CSMA (Saturated Traffic) . . . . . . 182

B.3 Goodput For Effective CSMA (Unsaturated Traffic) . . . . . 187

\begin{tabular}{|lll}
\hline Appendix C Additional Validation of Goodput Model & 189
\end{tabular}

C.1 Simulation (Two-ray Model and 48 Mbps) . . . . . . . . . . . 189

C.1.1 Saturated Traffic Demand . . . . . . . . . . . . . . 191

C.1.2 Unsaturated Traffic Demand . . . . . . . . . . . . . 192

C.2 Simulation (Two-ray Shadowing Model and 48 Mbps) . . . . 195

C.2.1 Saturated Traffic Demand . . . . . . . . . . . . 195

C.2.2 Unsaturated Traffic Demand . . . . . . . . . . . . . 196

\begin{tabular}{|lll}
\hline Appendix D Investigating Fairness with Channel Assignment & 199
\end{tabular}

D.1 Comparison of Jain's Index . . . . . . . . . . . . . 200

D.2 Comparison of Starvation Link Ratio . . . . . . . . . . . . . . 201

D.3 Comparison of Highest-to-lowest Goodput Ratio . . . . . . . 202

D.4 Jain's Index vs. Starvation Link Ratio. . . . . . . . . . . . 203

D.5 Flow Starvation with Interference Set Selections . . . . . . . 204 


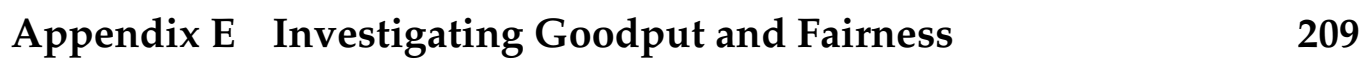

E.1 Simulation Environments . . . . . . . . . . . . . . . 209

E.2 Analysis of Fairness . . . . . . . . . . . . . . . . . . . . 210

E.3 Analysis of Goodput . . . . . . . . . . . . . . . . . . . . 211

E.4 Discussion ....................... 213

\section{Appendix F Additional Validation of Multi-objective Channel As-} signment 215

F.1 Validation of TUMOCA in Random Topology . . . . . . . . . 216

F.2 Validation of TAMOCA in Random Topology . . . . . . . . . 218

F.2.1 Validation of Traffic-aware Multi-objective Channel Assignment (heavy traffic) . . . . . . . . . . . . . 218

F.2.2 Validation of Traffic-aware Multi-objective Channe Assignment (medium traffic) . . . . . . . . . . . 219 


\section{List of Abbreviations}

$\begin{array}{ll}\text { WBN } & \text { Wireless Backhaul Network } \\ \text { WLAN } & \text { Wireless Local Area Network } \\ \text { CA } & \text { Channel Assignment } \\ \text { CSMA/CA } & \text { Carrier Sense Multiple Access Mechanism with Collision Avoidance } \\ \text { MAC layer } & \text { Medium Access Control layer } \\ \text { PHY layer } & \text { Physical layer } \\ \text { ITS } & \text { Intelligent Transportation System } \\ \text { VoIP } & \text { Voice over Internet Protocol } \\ \text { IPTV } & \text { Internet Protocol Television } \\ \text { PCS } & \text { Physical carrier sensing mechanism in IEEE 802.11 protocols } \\ \text { QoS } & \text { Quality of service } \\ \text { MCMR } & \text { Multi-channel multi-radio } \\ \text { SCSR } & \text { Single-channel single-radio } \\ \text { TUASCA } & \text { Traffic-Unaware Anti-Starvation Channel Assignment } \\ \text { TAASCA } & \text { Traffic-Aware Anti-Starvation Channel Assignment } \\ \text { TUMOCA } & \text { Traffic-Unaware Multi-Objective Channel Assignment } \\ \text { TAMOCA } & \text { Traffic-Unaware Multi-Objective Channel Assignment } \\ \text { SIR } & \text { Signal-To-Interference-Ratio } \\ \text { SINR } & \text { Signal-to-Interference-and-Noise-Ratio } \\ \text { ILP } & \text { Integer Linear Programming }\end{array}$




\section{List of Notations}

E Complete set of links in an IEEE 802.11 WBN

$N \quad$ The number of links in the set of $E$

$M \quad$ The set of available channels for $E$

$R_{c s} \quad$ Carrier sensing range

$D_{t r}^{\max } \quad$ Maximum transmission range

$D_{t r} \quad$ The distance between the sender and receiver in a link

IS (i) The independent set: the links out of $R_{c S}$ of a given link $i$

$\gamma(i) \quad$ The conflict set: the links within $R_{c S}$ of a given link $i$

$\chi(i) \quad$ The number of links in the IS $(i)$

$d_{s, s} \quad$ The distance between senders in two links

$d_{s, r} \quad$ The distance between sender and receiver in two links

$d_{l, L B} \quad$ The distance between a link $l$ and the left border link $L B$

$d_{l, R B} \quad$ The distance between a link $l$ and the right border link $R B$

$G_{C S}$ The left border link set in an IEEE 802.11 WBN

$G C S_{R B}$ The right border link set in an IEEE 802.11 WBN

$G_{C S}{ }_{L B}$ The dominant left border link set in an IEEE 802.11 WBN

$G_{C S^{\prime}}{ }_{R B}$ The dominant right border link set in an IEEE 802.11 WBN

$G_{C S}$ The middle-link set in an IEEE 802.11 WBN

$\gamma^{G M C}$ The minimum global conflict clique

$D \quad$ The distance between two border links in an IEEE 802.11 WBN

d The inter-link distance interval

$f(i) \quad$ The normalized traffic demand of a link $i$ 
$G(i) \quad$ The estimate of goodput for a link $i$ in effective CSMA

$G_{O}(i) \quad$ The optimistic estimate of goodput for a link $i$ in ineffective CSMA

$G_{P}(i) \quad$ The pessimistic estimate of goodput for a link $i$ in ineffective CSMA

GIS $_{L B} \quad$ The initial global left border set

GIS $_{R B} \quad$ The initial global right border set

GIS $_{L B} \quad$ The global left border set

GIS $_{R B} \quad$ The global right border set

GIS $_{M L} \quad$ The global middle-link set

$\gamma_{L}(i) \quad$ The local interference set of a given link $i$

$\beta \quad$ Path loss exponent factor

$\alpha \quad$ The Starvation factor for flow starvation

$R_{I} \quad$ Interference range

$S I R_{\text {threshold }}$ The threshold for Signal-To-Interference-Ratio (SIR) 


\section{Chapter 1}

\section{Introduction}

Wireless backhaul networks (WBNs) have emerged as a practical solution for bridging small-cell networks and backbone networks in lieu of wired backhaul networks [24,61,142,157] when it is difficult to install wired links. Such WBNs utilise wireless communications systems to connect an access point or end user to a major network or backbone network such as the Internet or the proprietary network of a large business, academic institution or government agency [61, 142].

The IEEE 802.11 protocols are an appealing choice for WBNs due to cost-efficient deployment, flexibility, and easy installation and maintenance, especially when an operator has a limited budget for the use of dedicated links [43, 49]. In recent years, due to low cost and easy installation, some new application scenarios have emerged in IEEE 802.11 WBNs such as smart grids and intelligent transportation systems [56, 97]. The similarity among these new applications is that they appear in built environments, such as the offices of the central business district (CBD), subways, or urban residential areas. These environments have a structured topology like a line or a grid (see Figure 1.1). These densely populated built environments bring greater needs for high Internet speed and stable transmission [26, 83, 84, 112, 131]. Therefore, IEEE 802.11 WBNs are facing unprecedented challenges to improve quality of service (QoS) in built 
environments with limited wireless resources [26, 83, 84, 98, 105, 147, 153].

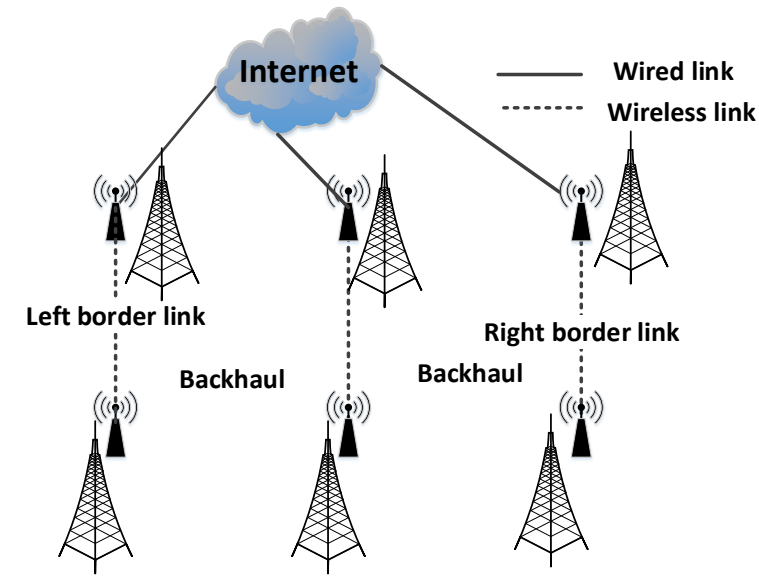

Figure 1.1: An illustration of a point-to-point wireless backhaul network in a linear layout

Ensuring QoS in a WBN using the IEEE 802.11 protocols has its unique set of challenges. The IEEE 802.11 WBN in a densely populated environment may experience path loss and shadowing, multi-path fading, which causes signal degradation and affects quality of service [6, 96]. Moreover, the unlicensed frequency bands and broadcast channels used in the IEEE 802.11 protocol suffer from interference and potential unfairness due to the placement of nodes causing flow starvation (extremely low goodput) [50, 73, 90, 132]. These performance issues lead to poor goodput and fairness in IEEE 802.11WBNs [6, 61, 89].

Some of the performance issues in IEEE 802.11 WBNs can be traced back to poor network planning [23,120]. For example, existing IEEE 802.11 WBN planning is that planning strategies mainly focus on maintaining coverage within some budget constraints [112, 146, 154]. Such planning strategies do not explicitly consider the QoS requirements and may fail to support QoS requirements for new-found uses such as smart grids and intelligent transportation systems. Another weakness of poor WBN plan- 
ning is that existing goodput models are not designed specifically for guiding the IEEE 802.11 WBN deployment in built environments.

To improve IEEE 802.11 planning, an accurate goodput model is essential by predicting the behaviour of wireless links in IEEE 802.11 WBNs [145]. A channel assignment is another key element of the planning in IEEE 802.11 WBNs that is capable of allocating channels to radios [6, 133]. A channel assignment is the fundamental part of the medium access control (MAC) layer and network layer to ensure fair sharing of channel resources among nodes in IEEE 802.11 WBNs [39,113, 115, 123], which is essential to higher layer protocols. When a channel assignment sufficiently utilises the channel resource in IEEE 802.11 WBNs, it can improve spatial reuse as well as present an optimal network topology to other communication protocols like routing and scheduling [5, 25, 112]. Such research will also help fulfil the expectation of high QoS in built environments of IEEE 802.11 WBNs.

This research extends the state of the art by designing a new goodput model and optimising channel assignment to improve network performance in IEEE 802.11 WBNs planning stage. Through goodput modelling and optimising channel assignment, this research contributes to better management of channel resources, better goodput and fairness to network users, and the ability to meet higher requirements in the future.

\subsection{Research Goals}

The goal of this research is to improve network performance (fairness and goodput) for planning IEEE 802.11 WBNs through goodput modelling and optimising channel assignment.

To achieve the research goal, this research investigates the potential to improve network performance by designing a goodput model and developing channel assignment algorithms in IEEE 802.11 WBNs. More specifically, this research helps answer the following questions: 


\section{- Goodput model:}

How can a goodput distribution model provide an accurate prediction of the behaviour of wireless links in a IEEE 802.11 WBN under various scenarios such as exposed nodes, hidden nodes and border effect?

- Channel assignment:

How can a channel assignment algorithm be designed to prevent flow starvation and improve fairness in IEEE 802.11 WBNs?

How can a channel assignment algorithm be designed to achieve predefined QoS requirements including fairness and goodput in IEEE 802.11 WBNs?

\subsection{Contributions}

This work contributes to goodput modelling and channel assignment in IEEE 802.11 WBNs. The contributions are listed as follows.

- A novel goodput distribution model providing accurate prediction of the link-level goodput in IEEE 802.11 WBNs. Such a model considers geometry topology, different carrier sensing scenarios, and traffic demands. This goodput model is the representative of IEEE 802.11 protocol and WBNs and also the fundamental basis of planning and optimising IEEE 802.11 WBNs.

- Traffic-unaware and traffic-aware channel assignment algorithms effectively preventing flow starvation and improving system fairness in IEEE 802.11 WBNs. A new traffic-aware interference model based on the new goodput model reflecting global interference (border effect) and local interference with respect to traffic load in IEEE 802.11 WBNs. 
- Traffic-unaware and traffic-aware multi-objective channel assignment algorithms using new fitness functions to help guide network designers with a set of feasible solutions. These feasible solutions guarantee to achieve predefined QoS requirements including fairness and average goodput for a given IEEE 802.11 WBN. Traffic-unaware and traffic-aware fitness functions based on the new goodput model evaluating the potential performance of the output from channel assignment algorithms in a given IEEE 802.11 WBN.

All the above contributions help predict link quality and manage channel resource to improve fairness and goodput for planning IEEE 802.11 WBNs.

\subsection{List of Publications}

The following publications relate to the discussion in Chapter 3:

- Y. Qu, B. Ng, The effect of carrier sensing mechanisms on wireless mesh network goodput, in: Proceedings of International Telecommunication Networks and Applications Conference (Sydney, Australia, 2015), ITNAC'15, IEEE, pp.106-113.

- Y. Qu, B. Ng, W. K. G. Seah, A goodput distribution model for IEEE 802.11 wireless mesh networks, in: Proceedings of the 34th International Performance Computing and Communications Conference (Nanjing, China, 2015), IPCCC'15, IEEE, pp. 1-8.

- Y. Qu, B. Ng, M. Homer, A Goodput Distribution Model for Planning IEEE 802.11 WBNs in Built Environments, Journal of Network and Computer Applications, Elsevier 99 (C) (2017) 28-46.

- Y. Qu, B. Ng, D. Singh, and L. Hao, Wireless backhaul performance with IEEE 802.11, accepted by the International Conference on Telecom- 
munications and Communication Engineering (OSAKA, Japan, 2017), ICTCE'17, ACM.

- Y. Qu, B. Ng, Goodput in wireless backhaul networks using IEEE 802.11: A MAC Irregularity perspective, Journal of Communications, Wiley-Blackwell (Under review)

Goodput in wireless backhaul networks using IEEE 802.11: A MAC Irregularity perspective The following publications are partially derived from the work presented in Chapter 4.

- Y. Qu, B. Ng, H. Yu, Anti-starvation channel assignment with global conflict set selection in IEEE 802.11 WMNs, in: Proceedings of the 12th ACM Symposium on QoS and Security for Wireless and Mobile Networks (Malta, Malta, 2016), Q2SWine'16, ACM, pp. 103-110. (Best poster award in MSWiM 2016)

- Y. Qu, B. Ng, W. Seah, A survey of routing and channel assignment in multi-channel multi-radio WMNs, Journal of Network and Computer Applications, Elsevier 65 (C) (2016) 120-130.

- Y. Qu, B. Ng, M. Homer, Optimisation of Channel Assignment For Improving Fairness in IEEE 802.11 WMNs, Journal of Computer Networks, Elsevier 129 (1) (2017) 215 - 231.

The following publication is partially derived from the work presented in Chapter 5.

- Y. Qu, B. Ng, H. Yu, P. Andreae, W. Seah, Fitness evaluation for channel assignment algorithms in IEEE 802.11 WMNs, in: Proceedings of the 14th Annual Consumer Communications \& Networking Conference (Las Vegas, USA, 2017), CCNC'17, IEEE pp.361-364.

The papers listed above have additional authors, the principal work and authorship was by the author of this thesis. 


\subsection{Organisation of Thesis}

This thesis has seven chapters and is organised as follows.

- Chapter 1: Introduction

Problem statement, research goal, research questions, thesis contributions, publications, and the thesis organisation are presented in this chapter.

- Chapter 2: Literature Review

This chapter presents a review of the literature about wireless backhaul networks, goodput modelling, and channel assignment. It highlights the main limitations and current challenges that form the motivations of the thesis.

- Chapter 3: Goodput Modelling

In this chapter, a new goodput distribution model is proposed. This model has been validated with various traffic demands and two propagation models.

- Chapter 4: Channel Assignment For Improving Fairness in IEEE 802.11 WBNs

In this chapter, a novel traffic-aware interference model is proposed. Two anti-starvation channel assignment algorithms are developed to prevent flow starvation and improve fairness in IEEE 802.11 WBNs under saturated and unsaturated traffic assumptions. The performance of two proposed channel assignment algorithms are validated through simulations.

- Chapter 5: Multi-objective Optimisation of Channel Assignment For Improving Fairness and Goodput in IEEE 802.11 WBNs 
In this chapter, two fitness functions and two new multi-objective channel assignment algorithms are developed to find a set of feasible solutions to achieve the predefined requirements including fairness and average goodput under saturated and unsaturated traffic assumptions. The performance of two proposed channel assignment algorithms is validated through simulation.

- Chapter 6: Conclusions

In this chapter, the conclusions and findings in each chapter have been presented and summarised. It also describes the main future research directions arising from the contributions of this work.

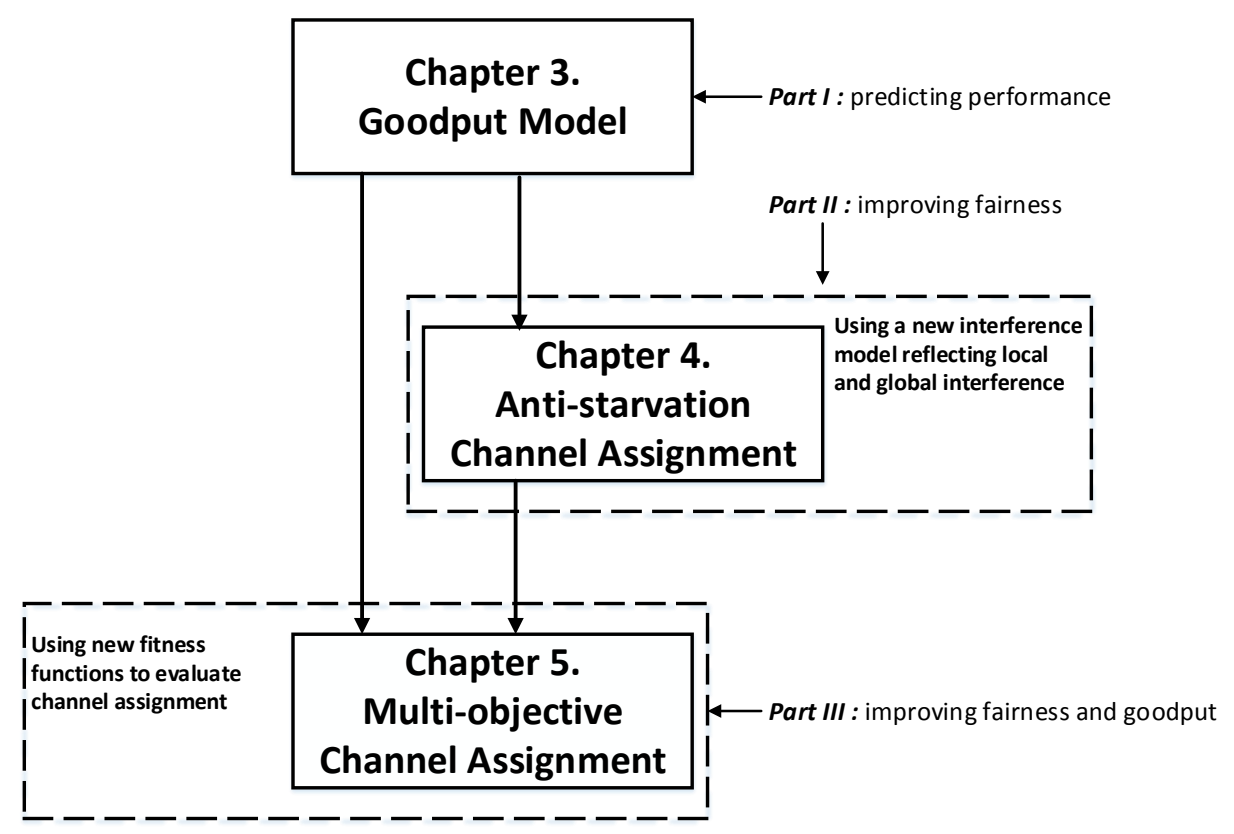

Figure 1.2: The connection between major contribution chapters in the thesis

The connection between the major contribution chapters is shown in this thesis in Figure 1.2. The goodput model in Chapter 3 is the funda- 
mental contribution that provides the prediction of performance in IEEE 802.11 WBNs. Chapter 4 investigates flow starvation with existing interference models in channel assignment algorithms and designs a new interference model based on the goodput model in Chapter 3. Using the new interference model, anti-starvation channel assignment algorithms improve fairness by preventing flow starvation. Chapter 5 investigates the trade-off between fairness and goodput with channel assignment algorithms. New fitness functions are developed based on the goodput model in Chapter 3. Using the new fitness functions, multi-objective channel assignment algorithms improve both fairness and goodput for IEEE 802.11 WBNs planning. 


\section{Chapter 2}

\section{Related Work}

This research investigates approaches to improve goodput and fairness in IEEE 802.11 wireless backhaul network (WBN) planning. This chapter starts with the definitions of key terms in this research and then introduces IEEE 802.11 WBNs including background, performance issues, and planning problems. Then a survey of goodput modelling and channel assignment (CA) for planning IEEE 802.11 WBNs is presented. This chapter summarises the issues in goodput modelling and CA that motivate this research.

\subsection{Definitions of Key Terms}

This section introduces the definitions of some key terms used through the whole thesis to facilitate the discussion.

- Quality of Service (QoS): From the perspective of a network, QoS is to provide a certain level of assurance that its traffic and service requirements can be satisfied [101]. Such QoS manages bandwidth based on the traffic demands and the configuration of network setting. Thus, the term "QoS" may include different aspects, ranging from the user's expectation to the carriers' requirements. This research studies two common aspects of QoS: goodput and fairness. 
- Goodput: In this thesis, goodput is used interchangeably with throughput. Such "goodput" refers to the actual achieved successful packets measured over the available links [7]. Goodput can be measured from the view of over a network or an end-to-end flow.

- Fairness: In the context of IEEE 802.11 WBNs, fair refers to that all nodes attain the fair media access, fairly share the bandwidth, the QoS requirements of all nodes are equally satisfied [131]. Different definitions of fairness have been used in the literature for wireless networks. According to a survey of fairness in [131], fairness can be classified as short-term and long-term fairness, and system and individual fairness. Short-term fairness refers to the resource allocation during a short time period while long-term fairness focuses on the fair resource allocation during a longer time period of the life time of a system. System fairness is observed from the perspective of a whole system regarding the overall fairness among all nodes. Individual fairness shows whether the system treats a certain node fairly according to the traffic demand of this node. This research focuses on studying long-term and system fairness in IEEE 802.11 WBNs.

- Flow starvation: In the context of IEEE 802.11 WBNs, flow starvation results from the border effect attributable to node placement. Border effect refers to the border links dominating the transmission in an IEEE 802.11 WBN [54]. Because the border links only have neighbouring links on one side, these border links experience less channel contention and are more likely to transmit more packets than the middle links in between border links. Middle links have neighbouring links on both sides and thus some middle links between the border links may experience extremely low goodput (what is commonly called flow starvation). Flow starvation significantly impacts network fairness [54].

- IEEE 802.11 carrier sensing mechanism: The IEEE 802.11 standard 
specification [2] mandates the use of a carrier sense multiple access mechanism with collision avoidance (CSMA/CA), which is also called physical carrier sensing. Physical carrier sensing detects any ongoing transmission by listening to the physical medium. If a listening node detects carrier signal strength greater than a predefined threshold, Carrier Sense Threshold, it defers its transmission and waits for some back-off time until the channel is sensed idle. Note that this research focuses on studying physical carrier sensing using Distributed Coordination Function (DCF) and does not consider virtual carrier sensing mechanism.

\subsection{IEEE 802.11 Wireless Backhaul Networks}

IEEE 802.11 WBNs are a popular backhaul technology in backhaul networks that utilise telecommunication networks to carry data between an access point and backbone networks [43]. In recent years, cellular networks dominate telecommunication networks all over the world [44, 65]. With the rapid development of data-hungry applications and increasing growth of traffic data, an efficient approach is to combine macro-cell cellular networks with small-cell cellular networks to improve QoS and provide satisfactory users' experience [4, 35, 43]. Such small-cell cellular networks provide coverage extension at some blind spots and enhance capacity to ease congested macro-cell networks with the advantage of low cost and easy installation [119].

With the increasing growth of small-cell networks, a backhaul solution consequently needs to serve a large amount of small-cell stations delivering data between small cells and backbone networks [4, 119]. Generally, these small-cell networks are deployed closer to end users since most of the new data is being generated indoors e.g. in built environments such as residential areas, subways, and offices [119,157]. Hence, it may be challenging to install wired links in these densely populated built environ- 
ments, where there are likely many obstacles such as buildings [56, 97].

IEEE 802.11 WBNs are an appealing choice for bridging small cell networks and backbone networks in lieu of wired backhaul networks in densely populated built environments [119]. Compared with dedicated solutions such as microwave links or satellite providing high capacity and reliability with high cost [142], IEEE 802.11 WBNs have many advantages and been widely applied to small-cell networks due to its low cost, ease of installation and maintenance, operation in the unlicensed band, and reasonable coverage range, especially when an operator has a limited budget for the use of dedicated links [43,49].

\subsubsection{Architecture and Application Scenarios in IEEE 802.11 WBNs}

IEEE 802.11 WBNs can be classified into two main types of architecture: (i) point-to-point IEEE $802.11 \mathrm{WBNs}$, and (ii) point-to-multipoint IEEE 802.11 WBNs [4, 157]. Point-to-point IEEE 802.11 WBN provides the link only between two nodes (see Figure 2.1). The point-to-point architecture is conventionally used for the non-line-of-sight scenarios. These scenarios usually use dedicated connections such as licensed spectrums to reduce interference [4].

Point-to-multipoint architecture is deployed for the line-of-sight scenarios [4]. In a point-to-multipoint IEEE 802.11 WBN, one node provides backhaul for several nodes, which looks like a hub connecting multiple clients (see Figure 2.2). This architecture brings more flexibility in topology than point-to-point architecture [4,157]. Licensed and unlicensed spectrum have been used in both point-to-point and point-to-multipoint architecture. Hence, IEEE 802.11 WBNs can be used in both point-to-point and point-to-multi- point architecture [4, 157].

Over the last decade, IEEE 802.11 WBNs have been rapidly developing and widely applied to small-cell networks such as enterprise networks, 


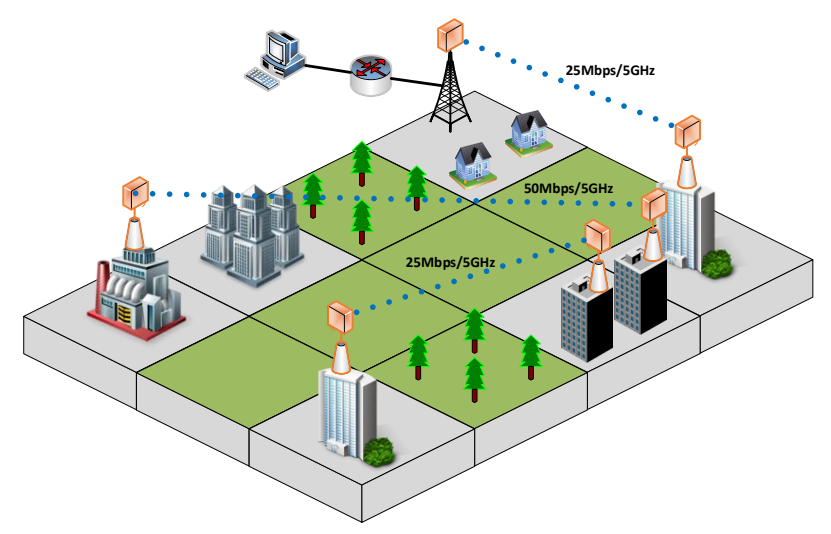

Figure 2.1: An example for a point-to-point WBN

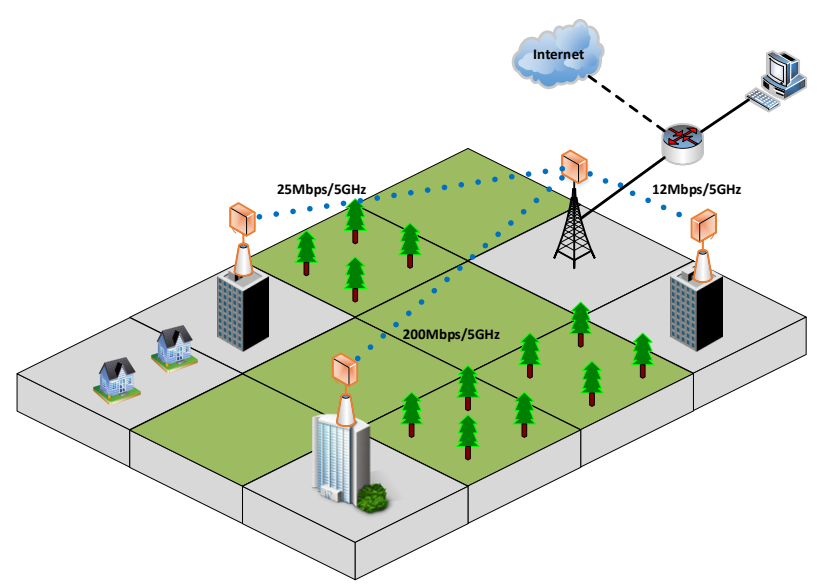

Figure 2.2: An example for a point-to-multipoint WBN

community networks, intelligent transport system networks, smart grids, and healthcare and medical system networks due to cost-efficient deployment [6, 38, 97, 142]. These applications are typically deployed in a structured topology such as a line or a grid topology [4]. The grid topology naturally occurs in built environments such as roads, railway tracks, and buildings. Some studies concluded that this natural occurrence of grid placement outperforms arbitrary placement in terms of coverage, connectivity, and fair capacity allocation [16, 129]. 
However, deployment in IEEE 802.11 WBNs still has its challenges [4, 43, 119, 151]. First, it is difficult to ensure QoS in high density environments with high population and various obstacles. Second, these applications involve various traffic types with different requirements such as voice calls, Internet Protocol television(IPTV) [69], and Voice over Internet Protocol (VoIP) [3]. For example, IPTV needs to consider the delay which impacts users experience. Therefore, the variability of traffic raises substantial challenges to IEEE 802.11 WBNs [6, 26, 83, 84].

The next sections will discuss the performance issues and network planning in IEEE $802.11 \mathrm{WBN}$.

\subsubsection{The Performance Issues and Network Planning in IEEE 802.11 WBNs}

With increasing volumes of data exchanged over networks and the need to better interconnect small-cell networks, IEEE 802.11 WBNs will play a more significant role in the coming years [119]. Serving the small-cell networks in built environments, where the traffic-growth leads to congestion, IEEE 802.11 WBNs need to not only extend coverage but also effectively manage the limited channel bandwidth among links to achieve the satisfactory level of goodput and fairness [6]. Users expect that all nodes or links achieve desired goodput that is closed to the traffic demand [84]. In addition, users expect fair sharing channel resource so that IEEE 802.11 WBNs needs to maintain a long term system fairness [6]. Moreover, the IEEE 802.11 WBN must not form a bottleneck of end-to-end connections in built environments [83, 84].

However, ensuring QoS in a WBN using the IEEE 802.11 protocol has its unique set of challenges. First, the unlicensed frequency bands and broadcast channels used in the IEEE 802.11 protocol suffer from interference, which significantly influences goodput and fairness in IEEE 802.11 WBNs [61]. These issues are well documented in [50,61]. Second, the 
exposed nodes problem brings capacity contention that leads to severe goodput degradation [152]. The hidden nodes problem causes collision at the receiver nodes that result in the packet loss and low goodput [143]. Moreover, the border effect problem causes flow starvation (extremely low goodput in some links) that leads to severe unfairness [54]. For example, in the point-to-point WBN shown in Figure 1.1 on page 2 , the border links tend to have higher goodput due to lower contention levels, while links in the middle are susceptible to extremely low goodput [61].

All these performance problems are related to the network topology of an IEEE 802.11 WBN. Network topology is the arrangement of the various elements such as nodes and links of a network, which forms the layout of IEEE 802.11 WBNs. Several studies demonstrated that the performance of an IEEE 802.11 WBN is largely determined by its topology [16, 129]. Such a topology in the IEEE 802.11 WBN is determined in the planning stage. Therefore, an investigation about IEEE 802.11 WBN planning with network performance is needed [6].

Network planning for IEEE 802.11 WBNs has received very little attention compared with other wireless networks such as $3 \mathrm{G}$ cellular network [9, 18, 106]. It borrows strategies from other types of wireless network due to cost constraints and the operation in unregulated spectrum [29. 111, 119]. However, performance issues in IEEE 802.11 WBNs show that the strategies from other networks may not be suitable for planning IEEE 802.11 WBNs due to the differences in the medium access control (MAC) protocol in use [99]. Current practice of ad hoc placement of nodes in IEEE 802.11 WBNs may be able to improve the coverage but this strategy no longer suffices for the requirements of QoS in dense environments, such as networking in smart grids and intelligent transport systems [14]. In indoor placement of wireless local area network (WLAN), introducing a new access point may improve the wireless coverage and overall goodput [31]. However, adding a new access point does not necessarily translate to better fairness because the new access point introduces additional contention 
or perhaps may cause severe unfairness like flow starvation [64]. Therefore, the strategies from other networks are not suitable for planning IEEE 802.11 WBNs and thus it needs to further improve the planning to provide better goodput and fairness.

These above issues have spawned a renewed interest in improving IEEE 802.11 WBN planning with better performance. To address these issues, a mathematical model is essential for planning and optimising IEEE 802.11 WBNs [90]. A goodput model representing IEEE 802.11 protocols will be convenient for analysing the performance problems in IEEE 802.11 WBNs and improving protocol designs [145].

Another way to address these performance issues is to research CA [6. 112, 146]. Allocating channel resources, a CA manages topology, capacity, and interference in IEEE 802.11 WBNs [115]. An efficient CA in IEEE 802.11 WBNs can improve network capacity significantly with management of spatial reuse and interference and also optimise fairness by fairly sharing channel resource among links in IEEE 802.11 WBNs [6]. Therefore, a CA is a key element for planning IEEE 802.11 WBNs with high goodput and fairness.

The next sections will review existing goodput models and CA algorithms. This review will focus on how these research improve IEEE 802.11 WBN planning and fulfil the expectation of high goodput and fairness in built environments of IEEE 802.11 WBNs.

\subsection{Goodput Modelling For Planning IEEE 802.11 WBNs}

A suitable goodput model is crucial for planning IEEE 802.11 WBNs because such a model abstracts the essence of the network such as the behaviour of wireless links and shows the interrelationships between key factors [145]. Network planners use a goodput model to improve planning by predicting network performance before deploying the network [157]. 
Since a goodput model for planning IEEE 802.11 WBNs normally involves topology, carrier sensing mechanism, and traffic demand, we will review how existing goodput models consider topology, carrier sensing scenario, and traffic demand separately.

\subsubsection{Goodput Models Considering Structured Topology}

The performance of an IEEE 802.11 WBN depends on its topology [16, 129]. Moreover, node placement is an important task determined in the planning stage such as the number of nodes and the location of these nodes in an IEEE 802.11 WBN. Hence, network topology is essential for goodput modelling and network planning in IEEE 802.11 WBNs [6].

The recent developments in IEEE 802.11 WBNs have been closely linked to the emergence of small-cell networks which are typically deployed in a structured topology such as a line or a grid topology discussed in Section 2.2.1. Hence, IEEE 802.11 WBNs may take advantage of the structured topology and a goodput model for planning IEEE 802.11 WBNs needs to consider the structured layout in built environments [119]. However, existing goodput models do not explicitly take into account the geometries of a structured topology which leads to inaccuracies in goodput prediction [114, 116].

Several studies have developed goodput models with consideration of network topology [64, 90, 104, 149]. For example, the studies in [90, 104] utilise the topology information to select the interfering links and noninterfering links of a given link that is used to calculate the goodput of the given link. However, none of these goodput models focus on planning an IEEE 802.11 WBN in a structured built environment. Therefore, developing a goodput model tailored to a structured topology with better accuracy is desirable for IEEE 802.11 WBN planning in built environments. 


\subsubsection{Goodput Models Considering IEEE 802.11 CSMA Mechanism}

For planning purposes, a goodput model needs to consider the IEEE 802.11 carrier sensing mechanism because goodput and fairness in IEEE 802.11 WBNs rely heavily on the efficiency of the carrier sensing mechanism [48, 114, 159].

The IEEE 802.11 carrier sensing mechanism is designed to protect packet transmission under effective carrier sensing condition, where all nodes are within each other's carrier sensing range and sense each other clearly [2]. In effective carrier sensing scenarios, the carrier sensing mechanism manages the medium access and affects the bandwidth allocation and potential collision at sender and receiver nodes, which is directly related to goodput and fairness [59].

However, ineffective carrier sensing scenarios with exposed nodes and hidden nodes are still common in real-world applications of IEEE 802.11 WBNs [115, 144, 161]. In ineffective carrier sensing scenarios, not all nodes are within each other's carrier sensing range. The exposed nodes, hidden nodes, and border effect affect the protection of packet transmission from the carrier sensing mechanism, which leads to goodput degradation and unfairness [64, 90, 104, 149]. Therefore, a goodput model for planning IEEE 802.11 WBNs needs to consider both effective and ineffective carrier sensing cases to cover different scenarios.

A broad class of goodput models for IEEE 802.11 protocol under the effective carrier sensing condition is based on the disk-graph model [68] that has been widely used in modelling carrier sensing mechanism for decades [37, 75]. Such a disk-graph model is a pairwise model in that the interference between two nodes is determined by the distance between the two nodes. The disk in the disk-graph model can be regarded as the interference range of a given node. When two nodes are within each other's disk, they interfere with each other while when they are out of each other's 
disk, no interference exists between them.

The disk-graph model can be traced back to Bianchi's models [27, 28] and Cali's model [34] characterising goodput under the effective carrier sensing condition. Both Bianchi's and Cali's models are based on singlehop wireless networks. They found that goodput of the IEEE 802.11 WLAN is related to the number of active nodes and minimum contention window size.

Felemban et al. [57] refined Bianchi's model by considering channel state during the back-off period. Several others [45,55,94] extended Bianchi's model using IEEE 802.11 [2]. For these goodput models under the effective carrier sensing condition, they assumed that all nodes can share the channel capacity equally as they can all sense the signal from each other clearly. The goodput depends on the number of active nodes and the configuration parameters of CSMA.

For ineffective carrier sensing scenarios, several studies have developed goodput models with consideration of exposed nodes, hidden nodes, and border effect [62, 109, 110, 134, 137, 141, 160]. Some findings under the ineffective carrier sensing condition come to a similar conclusion to those findings from models under the effective carrier sensing condition i.e. goodput depends on the number of active nodes and the configuration parameters of CSMA. Even though these goodput models are designed for ineffective carrier sensing scenarios, they did not consider the structured topology explicitly rendering these models less useful for planning the deployment of IEEE 802.11 WBNs in built environments.

Within the same area of studying ineffective carrier sensing scenarios, some studies about goodput model considered topology in their models [30, 64, 72, 73, 90, 104, 127, 149]. These models express throughput of a given link as a proportion of the probability of a successful transmission. The probability of successful transmission for a given link depends on many relevant probabilities such as the probabilities of packet arrival of its neighbouring nodes. These studies introduce many unknown probabil- 
ities related to channel state and transmission, such as collision probability, access attempt probability, leading to very high computation complexity. Hence, it is difficult to apply these models to planning IEEE 802.11 WBNs.

Even though many goodput models have been studied for IEEE 802.11 MAC protocols, none of these goodput models is suitable for planning IEEE 802.11 WBNs in built environments. First, the models based on the disk-graph model are simple and easy to use but many studies pointed out that the disk-graph model is not accurate because this model defines the carrier sensing effect as a binary function of distance [59, 162]. This apparent binary outcome of the carrier sensing mechanism overly simplifies the interaction among neighbouring nodes so this disk-graph model cannot accurately reflect the actual behaviour of wireless links. Moreover, disk-graph model cannot explain the goodput pattern in ineffective carrier sensing scenarios with exposed nodes, hidden nodes, and border effect. Hence, the disk-graph model is not suitable for planning IEEE 802.11 WBNs.

Second, those models for ineffective carrier sensing scenarios are also not suitable for network planning as they either do not consider the structured topology or made unrealistic assumptions that all detailed flow level information is known in advance. In the network planning stage, it is not realistic to collect flow level information and also difficult to apply these models to network planning.

\subsubsection{Goodput Models Considering Traffic Demand}

Traffic demand is another critical factor for a goodput model because it influences resource management and network performance in IEEE 802.11 WBNs [115]. This goodput model must be generalisable to deal with various applications in network planning stage that matches with the realworld application scenarios.

Saturated traffic demand has been used in Bianchi's models [27, 28] and Cali's model [34] to simplify the analysis. To generalise the goodput 
model, other studies extended their studies with both saturated and unsaturated traffic demands [45, 55, 94]. However, the studies about goodput model with both saturated and unsaturated traffic demands did not consider the other two factors, structured topology and carrier sensing mechanism at the same time.

Since traffic demand in real-world applications varies across different domains, it would be desirable for a goodput model to be flexible with both types of demands. Moreover, a goodput model is desirable to be flexible for both saturated and unsaturated traffic demands with other key factors.

\subsubsection{Summary of Goodput Models}

A goodput model plays an important role in IEEE 802.11 WBN planning for ensuring QoS among heterogeneous services with increasing data demands. Simplicity, usability and accuracy of a goodput model are three elements common to a successful model for network planning. The accuracy of a goodput model in IEEE 802.11 WBNs is related to the three key factors discussed before (structured topology, effective and ineffective carrier sensing, and traffic demand).

From our survey of the literature, no goodput model has been studied specifically for planning an IEEE 802.11 WBN in structured built environments. Specifically, existing studies do not link the goodput model with three key factors discussed before (structured topology, effective and ineffective carrier sensing, and traffic demand). Moreover, they do not provide insights to network wide performance - i.e. the performance is evaluated on a single link or adjacent links only.

Among the above discussed goodput models, the disk-graph model is the simplest model but it is not suitable for planning WBNs due to inaccuracy. For improving IEEE 802.11 WBN planning, a simple and accurate goodput distribution model is needed to provide network-wide performance with consideration of various application scenarios. 


\subsection{Channel Assignment For Planning IEEE 802.11 WBNs}

IEEE 802.11 WBNs started with single-channel single-radio (SCSR) system due to the limitations of radio technology at the time [6]. In SCSR IEEE 802.11 WBNs, all nodes use one radio with a common broadcast channel. Therefore, channel capacity contention and interference remained a major drawback in SCSR IEEE 802.11 WBNs.

With the development of advanced radio technologies, multi-channel multi-radio (MCMR) functionalities at each node provide effective approaches to enhance network performance in IEEE 802.11 WBNs [40, 53, 100]. In MCMR IEEE 802.11 WBNs, each node has multiple radio interfaces. Radio interfaces on nodes may transmit simultaneously because each radio has its own physical (PHY) layer and MAC layers. Compared with SCSR, MCMR technologies significantly increase network capacity, provide flexible connectivity, and reduce the interference among neighbouring links [6]. However, IEEE 802.11 WBNs may perform poorly due to inefficient utilisation of the multiple available channels and the multiple radios at its disposal [67, 70].

Therefore, the protocols in MCMR IEEE 802.11 WBNs need more sophistication to regulate spatial resources and optimise network performance. Various approaches have been studied in MCMR IEEE 802.11 WBNs to optimise network performance, such as rate control [79, 80, 124], MAC layer enhancement [19, 21, 108], or cross-layer designs between routing, CA, and scheduling [22, 58, 76, 95, 146, 150]. However, these studies have not adequately addressed goodput and fairness from the aspect of spatial utilisation and this is an increasingly prevalent problem in dense WBN scenarios under heavy traffic conditions [46, 60, 78, 112, 113]. Hence, an investigation about spatial utilisation is necessary to improve performance in IEEE 802.11 WBNs.

Among the above approaches, a CA plays a key role in managing spa- 
tial utilisation [6]. It also affects wireless connectivity and channel bandwidth, and regulates fair sharing of a channel among nodes. Therefore, a CA is essential to higher layer protocols such as routing protocol and directly determines goodput and fairness in IEEE 802.11 WBNs [7, 39, 115, 123, 133]

The main goal of CA algorithm is to allocate channels to the interfaces at each node in order to maintain network connectivity and mitigate capacity contention and interference among co-channel links [71]. It is challenging to achieve connectivity and mitigate contention and interference together because these two objectives are conflicting [115]. The problem of CA has been shown to be a NP-hard problem [126].

To achieve maximum connectivity, a CA preserves the network connectivity in the topology such that any pair of two nodes within transmission range can communicate with each other if they are allocated a common channel [133]. However, co-channel links within carrier sensing range cannot transmit at the same time and have to share the channel capacity, which contributes to high capacity contention. Concurrent transmission using the same channel will cause collision by the interference from the links within the interference range. Capacity contention and interference lead to goodput degradation or unfairness. In this sense, a CA utilises the variety of channel to reduce capacity contention and interference among neighbouring links but it may reduce the network connectivity. Therefore, maximising network connectivity and mitigating contention and interference need to be balanced in CA [133].

This section will first introduce the category of CA algorithms in IEEE 802.11 WBNs and then discuss how CA algorithms are designed to achieve goodput and fairness.

\subsubsection{Channel Assignment in IEEE 802.11 WBNs}

This research focuses on studying static CA as it is the most common type in IEEE 802.11 WBN planning stage [71]. Static CA is one of the three 
types of CA and a simple approach to allocate channels. Such a static CA allocates a channel to a node over an extended duration (in terms or weeks or months) [133].

Under the category of static CA, CA algorithms can be further categorised into traffic-unaware and traffic-aware CA. This classification is based on whether the CA algorithm considers the traffic load information such as the effective bandwidth and traffic rate on the links. Trafficaware CA algorithms consider traffic load information and try to satisfy some constraints (such as bandwidth requirement) while traffic-unaware CA does not need to consider traffic [115, 133]. Traffic-unaware CA algorithms allocate channels to the radios at each node based solely on the distance between nodes in the topology [115, 133].

Both traffic-aware and traffic-unaware CA have their own advantages [115]. The advantage of considering traffic load is that traffic-aware CA algorithms can reinforce QoS support initiated by routing algorithms leveraging traffic load information as its inputs, constraints, and objectives. The advantage of not considering traffic is that the traffic-unaware CA simplifies the problem since fewer factors are considered. Hence, IEEE 802.11 WBN planning should utilise the advantages of traffic-unaware and traffic-aware $\mathrm{CA}$ algorithms achieving better fairness and goodput.

The next sections will discuss traffic-unaware and traffic-aware CA algorithms regarding goodput and fairness in IEEE 802.11 WBNs.

\subsubsection{Traffic-unaware Channel Assignment}

Traffic-unaware CA algorithms allocate channel by using two popular approaches, graph theory (clique-based method) and network partitioning [11, 133]. These two approaches guide CA with different strategies to maintain connectivity and mitigate interference.

Graph-theory-based CA algorithms allocate channel based on theoretical concepts such as unit disk graph, topology, and conflict graph [11,133]. These theoretical concepts are used to model the relationship between 
nodes and links in the network. A unit-disk graph [42] represents the intersection of circles with an identical radius. In a unit-disk graph, the distance between connected vertices is less than the predefined transmission range. Naïvely, one can draw a unit-disk graph without considering the channel used on each link. However, the unit disk graph is not suitable for MCMR scenarios because it cannot represent the different channels used by different communication nodes, and the frequency of channels is needed to establish network topology in MCMR IEEE 802.11 WBNs [133]. Building upon the simple unit-disk concept, topology models were introduced in [102]. The extension was to simply require two nodes to share a common channel in addition to being within transmission range of each other to be deemed as a valid link. The concept of a conflict graph introduced in [77, 133] is an intuitive method to model interference in IEEE 802.11 WBNs. A conflict graph is derived from network topology and depends on the following factors: (i) two links use the common channel; (ii) two links transmit at the same time; (iii) the Euclidean distance between two links; (iv) the interference model [133].

Figures 2.3, 2.4, and 2.5 illustrate the examples of unit-disk graph, topology graph and conflict graph. The unit-disk graph in Figure 2.3 shows that five links connect four nodes A, B, C, and D according to the geometric distance and transmission range. By considering channel information, three links exist among four nodes A, B, C, and D where two channels 1 and 2 are available and only node $A$ has two radios and other three nodes have one radio (see Figure 2.4). Figure 2.5 shows three vertices representing three links $\mathrm{AB}, \mathrm{AC}$, and $\mathrm{AD}$ that are from the unit-disk graph. Since links AC and CD using the channel 2 interfere with each other, one edge connects the two corresponding vertices in the conflict graph.

Based on the above definitions and examples, unit-disk graph is independent of channel information on each nodes while topology graph is determined by the channel information with the unit-disk graph. Conflict graph is built up on the topology graph and an interference model. 


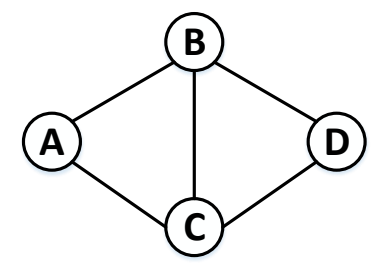

Figure 2.3: Unit-disk graph

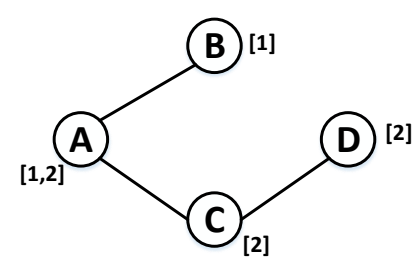

Figure 2.4: Network topology

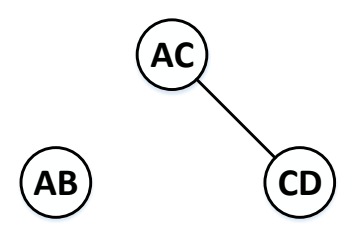

Figure 2.5: Conflict graph

All of these three graphs are the fundamental basis of graph-theory CA algorithms.

With the above three graphs, the common strategy in graph-theorybased CA algorithms is allocating channels to radios at each node to achieve a network topology that preserves every link in the unit disk graph or conflict graph, and reduces interference among the links in the conflict graph by distributing non-overlapping channels [133].

Network partitioning is another approach used in traffic-unaware CA algorithms. Partitioning is an intuitive method for solving the CA problem by dividing the network into disjoint sub-networks but this approach has its own issue [133]. The network-partition-based CA algorithms first divide the neighbouring nodes of a tagged node into $I$ groups, where $I$ is the number of radios at a given node. The second step is to allocate channels to each group. By using different channels in different sub-networks, these algorithms mitigate the interference among these sub-networks and maintain the connectivity among the links within a sub-network. Usually, each sub-network will select an edge node to maintain the connectivity between different sub-networks. However, the limitation of connectivity between different sub-networks appears to be problematic [133].

Next, traffic-unaware CA algorithms will be discussed from two aspects: design and evaluation. The design involves an objective and an interference model while the evaluation relies on the selected measurement metric. 
Table 2.1 shows the comparison of traffic-unaware CA algorithms according to the objective, interference model, and measurement metric. Four factors characterising the objective column in Table 2.1 are preserving connectivity (Con), maximising capacity (Cap), mitigating interference (Inter), and maintaining fairness (Fair). Preserving connectivity (Con) refers to the aim of providing maximum connectivity between nodes within a given transmission range subject to the constraint of limited radios and channels. The higher degree of connectivity among neighbouring nodes leads to more choices of paths available for routing. It also increases the reliability and robustness in IEEE 802.11 WBNs. The objective of maximising capacity (Cap) refers to the process of satisfying disparate traffic demands among competing nodes/flows. Basically, mitigating interference (Inter) means reducing the interference among the links in an inter-

Table 2.1: Comparison of traffic-unaware channel assignment algorithms

\begin{tabular}{|c|c|c|c|c|c|c|c|}
\hline \multirow{2}{*}{$\begin{array}{c}\text { Traffic-unaware } \\
\text { CA }\end{array}$} & \multicolumn{3}{|c|}{ Objectives } & \multirow{2}{*}{$\begin{array}{c}\text { Interference } \\
\text { Model }\end{array}$} & \multicolumn{3}{|c|}{ Evaluation } \\
\hline & Con & Cap & Inter & & G & FI & FS \\
\hline CLICA 102 & $\checkmark$ & & $\checkmark$ & Protocol & $\checkmark$ & & \\
\hline INSTC |140| & $\checkmark$ & & $\checkmark$ & Protocol & $\checkmark$ & & \\
\hline CTA [139] & $?$ & & $\checkmark$ & Protocol & $\checkmark$ & & \\
\hline BFS-CA [122] & $\checkmark$ & & $\checkmark$ & Protocol & $\checkmark$ & & \\
\hline IGSA [52] & $?$ & & $\checkmark$ & Protocol & $\checkmark$ & & \\
\hline NPS [126] & $\checkmark$ & & $\checkmark$ & Protocol & $\checkmark$ & & \\
\hline BFS-MaxNICA [138] & $?$ & & $\checkmark$ & Protocol & $\checkmark$ & & \\
\hline FBDCA 158 & $\checkmark$ & & $\checkmark$ & Protocol & $\checkmark$ & & \\
\hline SCCA [155] & $?$ & & $\checkmark$ & Protocol & $?$ & & \\
\hline TICA [36] & $?$ & & $\checkmark$ & Protocol & $\checkmark$ & $\checkmark$ & \\
\hline PCU-CA [88] & $?$ & & $\checkmark$ & Protocol & $\checkmark$ & & \\
\hline HCAP [92] & $?$ & & $\checkmark$ & Protocol & $\checkmark$ & & \\
\hline SSCA $[86$ & $?$ & & $\checkmark$ & Protocol & $\checkmark$ & & \\
\hline DGA 139] & $?$ & & $\checkmark$ & Protocol & $\checkmark$ & & \\
\hline CCCA [51] & $?$ & & $\checkmark$ & Protocol & $\checkmark$ & $\checkmark$ & \\
\hline
\end{tabular}


ference/conflict set. Maintaining fairness (Fair) refers to achieving the system fairness among the links in the whole network.

Interference model includes protocol interference model and measurementbased interference model (more discussion in Sections 2.4.2.2 and 2.4.3.2). Evaluation metrics include goodput (G), Jain's index [33] (FI), and flow starvation (FS). The metric FS refers to identifying the occurrence of flow starvation. A question mark is used to indicate that a CA does not explicitly contain the corresponding factor (but may use a surrogate factor that is comparable).

The next sections will review traffic-unaware CA algorithms from the objectives, interference models, and evaluation metrics.

\subsubsection{Objectives of Traffic-unaware Channel Assignment Algorithms}

Table 2.1 shows that traffic-unaware CA algorithms set their objectives towards preserving connectivity and mitigating interference and do not explicitly consider capacity and fairness requirement in their objectives.

Firstly, traffic-unaware CA algorithms preserve connectivity in different ways. Some algorithms aim to maximise connectivity. For example, Connected Low Interference Channel Assignment (CLICA) [102] preserves any link in the unit-disk graph and Minimum INterference Survivable Topology Control (INSTC) [140] generalises the approach in CLICA [102] with a $\mathrm{K}$-connected topology in a unit disk graph. Another way to preserve connectivity is to maintain the connectivity in the conflict graph such as Centralised Tabu-based Algorithm (CTA) [139] and Improved version of Gravitational Search Algorithm (IGSA) [52]. These two different strategies maximise the different level of connectivity but yield different performance because of the way constraints are expressed in their respective objective (i.e. optimisation over conflict graph vs. optimisation over unitdisk graph).

Among the graph-theory-based algorithms, the Breadth First Search Channel Assignment (BFS-CA) [122] is the first algorithm to preserve the 
global connectivity over the entire network by allocating a common channel at each node. Even though a common channel helps achieve global connectivity, using a common channel has the drawback that this BFSCA [122] is not flexible and strong co-channel interference exists among neighbouring links.

Different from graph-theory-based approaches, network-partition-based CA algorithms such as Neighbour Partitioning Scheme (NPS) [126] and Clique-based clustering channel assignment (CCCA) [51] preserve connectivity within a sub-network. Nodes in different sub-networks cannot communicate with each other directly. Therefore, CA algorithms based on network partitioning yield a poorer degree of connectivity in the network compared with graph-theory-based CA algorithms.

Secondly, traffic-unaware CA algorithms tend to mitigate total interference level among the links in the whole network through a three step process [115]. The first step is measuring the interference, then ranking the links based on the measured interference, and finally allocating different channels among the adjacent links in the conflict graph to reduce the interference. Most of these algorithms measure interference by using link conflict weight that is the number of the links in an interference set. The algorithms such as CLICA [102], CTA [139], and INSTC [140] compute the total number of edges with co-channels connecting the vertices in the conflict graph and work backwards to allocate channels for minimising interference. CA algorithms using this metric choose the least-used channel to reduce the total interference in the network. Some other CA algorithms select different interference metrics such as interference cost in Self-Stabilizing Channel Assignment (SSCA) [86] and Topology-controlled Interference-aware Channel Assignment (TICA) [36]. The interference cost used in SSCA [86] measures the interference level between two overlapping channels with in an interference set. The interference cost used in TICA [36] is calculated for each link as a function of distance. In all, these traffic-unaware CA algorithms reduce total interference lever among the 
links in the whole network that leads to high goodput.

Traffic-unaware CA algorithms using graph-theory (clique-based) methods and network partition are easy to understand and solve network flow problems. All surveyed traffic-unaware CA algorithms simplified the CA problem without considering traffic load.

These traffic-unaware algorithms aim to preserve connectivity and mitigate interference but did not explicitly consider maximising capacity and fairness in their objectives. Moreover, these algorithms do not take flow starvation into account. Therefore, traffic-unaware CA algorithms may not be able to achieve both goodput and fairness for IEEE 802.11 WBN planning.

Since these traffic-unaware CA algorithms mainly consider interference mitigation which is related to goodput in their objectives. The validation of these algorithms mainly focuses on average goodput or total goodput (see Table 2.1). Other similar metrics have been used to validate traffic-unaware CA algorithms such as delay, interference degree, packet delivery ratio. A few algorithms were validated with Jain's index [33]. However, none of these traffic-unaware CA algorithms identify whether flow starvation existed in the first place. Without identifying flow starvation, it is difficult to ascertain whether a CA algorithm resolves flow starvation and achieves desired fairness. Therefore, further optimisation and validation on traffic-unaware $C A$ algorithms are needed to to improve fairness and goodput for IEEE 802.11 WBN planning.

\subsubsection{The Interference Models Used in Traffic-unaware Channel As- signment}

An interference model is the key element in CA algorithms because whether the interference model is accurate or realistic directly influences the effectiveness of CA algorithms in achieving desired goodput or fairness [112]. CA algorithms use the interference model to select an interference set and estimate interference within the interference set. CA algorithms rely on the 
concept of an interference set to allocate available channels with the goal of reducing contention levels in channels and reducing interference among neighbouring link. The interference set of a link $i$ is the set of neighbouring links sharing a common channel and is within the interference range of link $i$ [115]. Generally, the interference range of a link is defined by the interference model used in CA algorithms. Therefore, the interference set and interference model are the key consideration in CA algorithms and significantly influences the channel allocation for improving the spatial reuse and the overall network performance.

Theoretical protocol interference model is widely used in traffic-unaware CA algorithms to determine the interference set [133] (see Table 2.1). Generally, theoretical protocol interference model defines the interference range of a receiver node represented as a disc (with radius $R_{i}=k \times D_{t r}$ ) around the receiver node where $D_{t r}$ is the transmission range and $k$ is a multiplicative constant. Widely documented theoretical models include protocol model, capture threshold model, and interference range model [75]. No packet transmission failures at a receiver node are assumed when its interfering nodes are out of the interference range [41]. Generally, theoretical models are not realistic because they only consider the factor of distance and ignore other factors such as transmission power level and the cumulative effect of interfering signals.

Even though the traffic-unaware CA algorithms are simple and have been widely used in IEEE 802.11 WBNs for decades, these CA algorithms still have some issues. For example, the protocol interference model may not be accurate and realistic. Hence, the traffic-unaware CA algorithms using the protocol interference model may estimate interference inaccurately and lead to poor goodput and fairness in IEEE 802.11 WBNs.

\subsubsection{Traffic-aware Channel Assignment}

Different from traffic-unaware CA algorithms, traffic-aware CA algorithms allocate channels by considering traffic information [133]. Hence, traffic 
patterns are important to traffic-aware CA algorithms. Two common traffic flow patterns, gateway pattern and peer-to-peer pattern, are used in traffic-aware CA to suit different scenarios. A gateway pattern assumes that most traffic flows are to or from gateway nodes. Unlike the gateway pattern, peer-to-peer pattern makes no assumption on the traffic flow patterns and is more widely applicable. Utilising the gateway pattern simplifies the CA algorithm but applying the CA algorithm for the gateway pattern to a scenario with the peer-to-peer pattern may not yield a solution (non-satisfiability) [133]. In contrast, the CA for the peer-to-peer pattern is more flexible. This research focuses on peer-to-peer traffic pattern that is more suitable for planning IEEE 802.11 WBNs to suit different scenarios.

Table 2.2 compares traffic-aware CA algorithms with the same table headings identical to Table 2.1 and the explanation appears in Section 2.4.2.

The next sections will review traffic-aware CA algorithms from their objectives, interference models, and evaluation metrics in the validation separately.

\subsubsection{Objectives of Traffic-aware Channel Assignment Algorithms}

Table 2.2 shows that most surveyed traffic-aware CA algorithms have their objectives set towards maximising capacity (goodput) and mitigating interference but maximising both goodput and fairness has not drawn enough attentions [7, 12, 115, 133].

First, to maximise capacity and mitigate interference without consideration of fairness, the algorithms such as Cluster-based Multipath Topology control and Channel assignment (CoMTaC) [107] and Joint Optimal Channel Assignment and Congestion Control (JOCAC) [121] attempt to maximise capacity by reducing the interference in the network. By choosing the channel with the least aggregated traffic load or least interference cost, these algorithms aim to mitigate interference and achieve the highest available channel capacity or the capacity greater than traffic demands. These algorithms only target goodput and neglect fairness. 
Table 2.2: Comparison of traffic-aware channel assignment algorithms

\begin{tabular}{|c|c|c|c|c|c|c|c|c|}
\hline \multirow{2}{*}{$\begin{array}{c}\text { Traffic-unaware } \\
\text { CA } \\
\end{array}$} & \multicolumn{4}{|c|}{ Objectives } & \multirow{2}{*}{$\begin{array}{c}\text { Interference } \\
\text { Model }\end{array}$} & \multicolumn{3}{|c|}{ Evaluation } \\
\hline & Con & Cap & Inter & Fair & & G & FI & FS \\
\hline LA-CA $\mid 126$ & $?$ & $\checkmark$ & $\checkmark$ & $?$ & Protocol & $\checkmark$ & & \\
\hline BSCA [87] & $?$ & $?$ & $?$ & $\checkmark$ & Protocol & $\checkmark$ & & \\
\hline RCL [8] & $?$ & & $?$ & $\checkmark$ & Protocol & $\checkmark$ & & \\
\hline MCI-CA 32 & $?$ & & $\checkmark$ & $?$ & Protocol & $\checkmark$ & & \\
\hline MCAR |13] & $?$ & $\checkmark$ & $\checkmark$ & $?$ & Protocol & $\checkmark$ & & \\
\hline MVCRA-R |15] & $?$ & $\checkmark$ & $\checkmark$ & $?$ & SINR & $\checkmark$ & & \\
\hline BCS [93] & $?$ & $\checkmark$ & $\checkmark$ & & Protocol & $\checkmark$ & & \\
\hline Hyacinth |125] & $?$ & & $\checkmark$ & & Protocol & $\checkmark$ & & \\
\hline DMesh 47 & $?$ & $\checkmark$ & $\checkmark$ & & Protocol & $\checkmark$ & & \\
\hline CoMTaC [107] & $?$ & $\checkmark$ & & & Protocol & $\checkmark$ & & \\
\hline ITACA $|128|$ & $?$ & & $\checkmark$ & & Protocol & $\checkmark$ & & \\
\hline G-PaMeLA [63] & $?$ & & $\checkmark$ & $\checkmark$ & Protocol & $\checkmark$ & $\checkmark$ & \\
\hline JOCAC |121| & $?$ & $\checkmark$ & $\checkmark$ & & SINR & $\checkmark$ & & \\
\hline TARICA [148] & $?$ & & $\checkmark$ & & Protocol & $\checkmark$ & & \\
\hline EXT-HMCP [85] & $?$ & & $\checkmark$ & & Protocol & $\checkmark$ & $\checkmark$ & \\
\hline MIN-INT [85] & $?$ & & $\checkmark$ & & Protocol & $\checkmark$ & $\checkmark$ & \\
\hline MAX-CP & $?$ & $\checkmark$ & $\checkmark$ & & SINR & $\checkmark$ & $\checkmark$ & \\
\hline CRACA & $?$ & $\checkmark$ & $\checkmark$ & $?$ & Protocol & $\checkmark$ & $\checkmark$ & \\
\hline TMCA 67 & $?$ & $\checkmark$ & $\checkmark$ & & Protocol & $\checkmark$ & & \\
\hline OTACA [103] & $?$ & $\checkmark$ & $\checkmark$ & $?$ & Protocol & $?$ & $\checkmark$ & \\
\hline GTA [135] & $?$ & & $\checkmark$ & $?$ & Protocol & $?$ & & \\
\hline MPFBCA [20] & $?$ & $\checkmark$ & $\checkmark$ & $?$ & Protocol & $\checkmark$ & $?$ & \\
\hline
\end{tabular}

Some algorithms target maximising capacity with fairness consideration as an auxiliary constraint [13, 15, 32, 126]. For example, Load Aware Channel Assignment (LA-CA) [126] and Maxflow-based Channel Assignment (MCAR) [13] aim to satisfy traffic rate requirement for each link with a "bandwidth conservation" principle. A defining characteristic of the fairness optimisation is distributing the bandwidth equally subject to the "bandwidth conservation" principle. For links using a common chan- 
nel within an interference set, the bandwidth conservation principle limits the sum of allocated bandwidth below the channel capacity [8, 87, 133]. The bandwidth conservation principle is derived on the assumption that all nodes share the available capacity fairly. This constraint is valid in effective carrier sensing scenarios but it neglects the case of hidden nodes in ineffective carrier sensing scenarios. Therefore, the algorithms using this fairness constraint may lead to poor goodput and fairness in ineffective carrier sensing scenarios.

Second, the closest studies to support fairness in the objectives are the studies in [8, 19, 21, 63, 87, 121]. Some channel assignment algorithms such as Generalised Partitioned Mesh network traffic and interference aware channel assignment (G-PaMeLA) [63] and Maximising Per-Flow Bandwidth Channel Assignment (MPFBCA) [19] aim to achieve Max-Min Fairness (MMF). For MMF, the rate of each node or link cannot be increased without decreasing the rate of any other node or link at the same time [19. 21. 63]. Other algorithms such as Joint Routing, Channel Assignment, and Link Scheduling (RCL) [121] and Balanced Static Channel Assignment (BSCA) [87] related to fairness set their objectives as proportional fairness (PF). The goodput performance of algorithms targeting MMF is limited by the slowest link. In terms of PF, the studies surveyed impose a fairness constraint $\lambda$ to each flow. In terms of PF, the metric is a fairness constraint $\lambda=\frac{G_{\text {actual }}}{F_{\text {demand }}}$ for every node or link, where $G_{\text {actual }}$ and $F_{\text {demand }}$ denote the actual goodput and the traffic demand. These $\mathrm{CA}$ algorithms aim to achieve proportional fairness among flows instead of maximising the overall goodput [8, 87, 121].

Third, Traffic-aware CA algorithms preserve connectivity with different purposes since connectivity is not the main goal of these traffic-aware CA algorithms. These algorithms only maintain a certain level of connectivity for the links given with a traffic demand instead of maximising the connectivity in IEEE 802.11 WBNs and therefore question marks are put in Table 2.2. Some algorithms meet the requirement of instantaneous 
routing demands by trading off maximum connectivity. For example, the LA-CA [126], BSCA [87], and RCL [8] algorithms aim to solve the problem of CA combined with routing. LA-CA [126] uses an iterative method that repeats the process of $\mathrm{CA}$ and routing to find the solution until the allocated flow rate satisfies the traffic demand. However, if traffic demand is greater than the available link capacity, the LA-CA [126] algorithm does not converge in polynomial time. MCI-CA [32] formulates the CA as a Matroid intersection problem by finding a forest topology which is a sufficient condition for a stable scheduling algorithm to achieve interferencefree link transmission between adjacent links. However, it may not be possible for MCI-CA [32] to find feasible forest topology for all different scenarios. MCAR [13] formulates the CA problem as a single commodity flow problem to calculate the maximum throughput and the required amount of flow rate on each link. Based on the required amount of flow rate on each link, MCAR [13] divides links into groups and allocates different channels to different groups. Even though the interference problem between groups is addressed, the interference reduction within a group is not addressed [133].

Traffic-aware CA algorithms solve the channel allocation more realistically than traffic-unaware CA algorithms by taking traffic information into account, which also increase the complexity of formulating the problem [133].

Most of the surveyed traffic-aware CA algorithms target capacity allocation and interference mitigation as their objectives with fairness constraints. However, these traffic-aware CA algorithms did not explicitly consider both goodput and fairness in their objectives. Therefore, trafficaware CA algorithms may not be able to achieve desired goodput and fairness for IEEE 802.11 WBN planning.

Since these traffic-aware CA algorithms mainly focus on maximising goodput instead of maximising goodput and fairness, most of them have only been validated about the performance on achieving goodput (see Ta- 
ble 2.2). A few algorithms were validated with Jain's index. However, none of these traffic-aware CA algorithms identify whether flow starvation exists in the first place. Without identifying flow starvation, it is difficult to ascertain whether a CA algorithm resolves flow starvation and is able to achieve both goodput and fairness. Therefore, it is needed to further optimise and validate traffic-aware CA algorithms to improve fairness and goodput for IEEE 802.11 WBN planning.

\subsubsection{The Interference Models Used in Traffic-aware Channel Assign- ment}

Traffic-aware CA algorithms used theoretical and measurement-based interference models to select interference sets but these two models have some shortcoming to model flow starvation in ineffective carrier sensing scenarios. The theoretical interference models have been discussed in Section 2.4.2.2. Besides theoretical interference models, some CA algorithms such as Minimum Variation channel and rate reassignment algorithm (MVCRA-R) [15] and JOCAC [121] use measurement-based interference model, SINR model, that are based on the measure of received signal [121]. Measurement-based interference models evaluate interference at a receiver node by calculating signal-to-interference-and-noise-ratio (SINR) or signal-to-noise-ratio (SNR) [37]. When SINR or SNR measured at a receiver node is below a specific threshold, this receiver node probably cannot receive a correct packet and the transmission fails. This assumption is based on massive simulations for mapping SINR to bite error rate. Note that SINR threshold is defined based on different modulation and coding mechanisms in different applications [2].

The difference between a theoretical model and a measurement-based model is whether the interference model considers the interference power or the additive effect from multiple neighbouring links or not [75]. Theoretical models assume that interference is a binary or pairwise conflict effect among concurrent transmitting links without consideration of power 
and additive effect [75]. Measurement-based models regard interference as an additive effect from all other neighbouring links transmitting simultaneously since SINR calculates the sum of noise signal power and signal power from all interfering nodes [75]. In summary, theoretical models are simple but not realistic while measurement-based models are realistic but complicated. Different interference models yield different interference sets that lead to different channel allocations and fairness in IEEE 802.11 WBNs.

In all, these two models reflect the interference among neighbouring links within an interference set. However, these two models cannot reflect flow starvation caused by hidden/exposed nodes and border effects. Flow starvation introduces severe unfairness [61]. The inaccuracy in the interference models may lead CA algorithms to poor goodput and fairness in IEEE 802.11 WBNs.

\subsection{Summary}

IEEE 802.11 WBNs are a promising technology to provide high speed and reliable connectivity to small-cell networks with low cost and efficient installation. However, using unlicensed spectrum in high density environments leads to big challenges to plan IEEE 802.11 WBNs for achieving high goodput and fairness.

The performance issues discussed in this chapter show that current goodput model and CA still fall short of the requirements from IEEE 802.11 WBN planning, providing room for further improvement [112] (more discussions in Chapters 3, 4, and 5). First, existing goodput models are not suitable for planning IEEE 802.11 WBNs due to inaccuracy or complexity. Second, most existing CA algorithms focus on maximising goodput but fairness in WBNs has not received much attention. Especially, preventing severe unfairness like flow starvation has not been explicitly considered in CA and IEEE 802.11 WBN planning. Last but not least, optimising CA 
with both goodput and fairness is a timely research topic but it has not been addressed by existing CA algorithms.

The end goal of this research is to improve goodput and fairness for planning IEEE 802.11 WBNs through goodput modelling and optimised CA algorithms.

The next sections will discuss how to improve goodput and fairness through goodput modelling and optimised CA. 


\section{Chapter 3}

\section{Goodput Modelling for Planning IEEE 802.11 WBNs}

The purpose of this chapter is to develop a goodput distribution model for planning IEEE 802.11 WBNs. Such a goodput distribution model is used to predict link-level goodput and help network planners resolve the performance issues in IEEE 802.11 WBNs planning. Some of the performance issues such as goodput degradation and flow starvation in IEEE 802.11 WBNs serving small-cell networks can be traced back to poor network planning [23]. A suitable goodput model is needed for guiding the deployment of IEEE 802.11 WBNs to improve network performance.

Among the goodput models discussed in Chapter 2, the disk-graph model is the simplest model that has been widely used in performance modelling in IEEE 802.11 WBNs [37,75]. To characterise the disk-graph model, an investigation has been conducted under effective and ineffective carrier sensing scenarios through simulation (see Appendix A). The investigation shows that the goodput patterns in effective carrier sensing scenarios match with the expectations from the disk-graph model. When all nodes are within each other's carrier sensing range, these nodes share the channel capacity fairly. When all nodes are out of each other's carrier sensing range, each node occupies the whole channel capacity. 
However, some simulation results in ineffective carrier sensing scenarios show an unexpected goodput pattern from the disk-graph model. According to the disk-graph model, links within the carrier sensing range of a link should share channel capacity equally and the sum of goodput of the links in any interference set is below 1 . However, this conclusion does not hold in the scenarios with border effect and flow starvation. Hence, the disk-graph model cannot explain the flow starvation in the middle link and the interaction between border links and middle link. Hence, due to inaccuracy, the disk-graph model is not suitable for planning WBNs in built environments.

An opportunity exists to shed light on IEEE 802.11 WBN planning through a new goodput model to improve performance. Such a goodput model plays a role in IEEE 802.11 WBN planning by representing the essence of performance problem and reflecting the behaviour of wireless links in IEEE 802.11 WBNs. Moreover, this goodput model brings convenience for performance analysis and node placement.

Simplicity, usability and accuracy of a goodput model are three elements common to a successful model for network planning. In developing a goodput model, a good approach is to begin with a very simple version and then move towards more elaborate models that more nearly reflect the complexity of the real problem. The usability of a goodput model needs to satisfy the purpose of developing the model, and in this research, the purpose is about planning IEEE 802.11 WBNs. The accuracy of a goodput model is related to the three key factors influencing the performance in IEEE 802.11 WBNs: structured topology, effective and ineffective carrier sensing, and traffic demand (more discussion in Section 2.3 on page 18 ).

The goal of this research in this chapter is to develop a simple and accurate goodput distribution model for planning IEEE 802.11 WBNs (a metric that reflects network-wide performance) with consideration of a structured topology for various application scenarios.

This chapter explains the development of a goodput model for IEEE 
802.11 WBNs through a two step process. First, a goodput distribution is derived based on the disk-graph model to cover effective and ineffective carrier sensing scenarios. Such a goodput model predicts link level goodput based on network topology and traffic demands. Finally, this model is validated through simulations under different traffic demands and different radio propagation models that reflect typical built environments.

The objectives of this chapter are to:

1. Derive a goodput distribution model to predict link level goodput based on the topology of a given IEEE 802.11 WBN and traffic demand.

2. Validate the proposed goodput distribution model through simulations.

The rest of this chapter is organised as follows. Section 3.1 explains the derivation of the proposed goodput model. Sections 3.2 and 3.3 validate the model through simulation followed by the summary in Section 3.4 .

\subsection{A Unified Goodput Distribution Model For- mulation}

In this section, a unified goodput distribution model is developed for planning IEEE 802.11 WBNs in built environments. Such a goodput distribution model conserves the disk-graph model for effective carrier sensing scenarios and also extends to the unexpected goodput pattern in ineffective carrier sensing scenarios (see Appendix A). This model will also take the structured topology in built environments into account, and it will be flexible for both saturated and unsaturated traffic demands.

Several variables are defined to facilitate the discussion. The symbols for these variables together appear with a brief explanation in Table 3.1 . 
Table 3.1: Notation: symbols and their meanings.

\begin{tabular}{|c|c|}
\hline Symbol & Explanation \\
\hline E & Complete set of links in an IEEE $802.11 \mathrm{WBN}$ \\
\hline$N$ & The number of links in the set of $E$ \\
\hline$R_{c s}$ & Carrier sensing range \\
\hline$D_{t r}^{\max }$ & Maximum transmission range \\
\hline$D_{t r}$ & The distance between the sender and receiver in a link \\
\hline$I S(i)$ & The independent set: the links out of $R_{c s}$ of a given link $i$ \\
\hline$\gamma(i)$ & The conflict set: the links within $R_{\mathcal{C S}}$ of a given link $i$ \\
\hline$\chi(i)$ & The number of links in the IS $(i)$ \\
\hline$d_{s, s}$ & The distance between senders in two links \\
\hline$d_{s, r}$ & The distance between sender and receiver in two links \\
\hline$d_{l, L B}$ & The distance between a link $l$ and the left border link $L B$ \\
\hline$d_{l, R B}$ & The distance between a link $l$ and the right border link $R B$ \\
\hline$G C S_{L B}$ & The left border link set in an IEEE $802.11 \mathrm{WBN}$ \\
\hline$G C S_{R B}$ & The right border link set in an IEEE 802.11 WBN \\
\hline$G_{C S}^{\prime}{ }_{L B}$ & The dominant left border link set in an IEEE 802.11 WBN \\
\hline$G C S^{\prime}{ }_{R B}$ & The dominant right border link set in an IEEE 802.11 WBN \\
\hline$G C S_{M L}$ & The middle-link set in an IEEE $802.11 \mathrm{WBN}$ \\
\hline$\gamma^{G M C}$ & The minimum global conflict clique \\
\hline$D$ & The distance between two border links in an IEEE 802.11 WBN \\
\hline$d$ & The inter-link distance interval \\
\hline$f(i)$ & The normalised traffic demand of a link $i$ \\
\hline$G(i)$ & The estimate of goodput for a link $i$ in effective CSMA \\
\hline$G_{O}(i)$ & The optimistic estimate of goodput for a link $i$ in ineffective CSMA \\
\hline$G_{P}(i)$ & The pessimistic estimate of goodput for a link $i$ in ineffective CSMA \\
\hline$\alpha$ & The starvation factor for flow starvation \\
\hline
\end{tabular}

The next subsections will explain the proposed goodput distribution model with saturated and unsaturated traffic demands separately. To help understand the new goodput model, examples will be provided to explain how to use this model to calculate goodput distribution with a given topology and traffic demand. 


\subsubsection{A Goodput Distribution Model For Saturated Traffic Demand}

The formulation for goodput model with saturated traffic is based on the disk-graph model and our previous work [114].

First, the assumptions for the model with saturated traffic demand are listed as follows:

Assumption 1. All nodes are configured with identical parameters and saturated traffic is assumed.

Assumption 2. A single-channel single-radio IEEE 802.11 WBN is configured with a linear uniform and symmetric topology.

Assumption 3. If the distance between two border links is less than the carrier sensing range, this scenario is regarded as the effective carrier sensing scenario. All links share the channel capacity equally in this effective carrier sensing scenario.

Assumption 4. If the distance between two border links exceeds the carrier sensing range, this scenario is regarded as the ineffective carrier sensing scenario and border effect exists.

Assumption 5. Starvation occurs to a link when the link is within carrier sensing range of two border links. In this research, a starvation link is defined as that

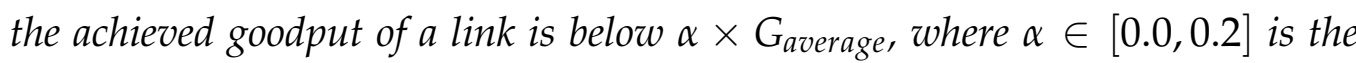
starvation factor and $G_{\text {average }}$ is the average goodput in $E$.

Assumption 6. The propagation delay between neighbouring nodes is zero.

Assumption 7. Capture effect and packet losses caused by collision are ignored.

Assumption 8. Acknowledgements are obtained instantaneously.

Assumptions 1 and 2 simplify an IEEE 802.11 WBN as a single-channel single-radio system with a structured topology and an identical configuration for all nodes. Assumptions 3, 4, and 5 are the conclusions that have 
been validated through well-known studies such as [27, 28, 61, 64, 90, 114]. Assumptions 6, 7, and 8 simplify the analysis by neglecting the propagation delay, capture effect and interference caused by collision.

The next section defines the goodput formulations for saturated traffic demand in effective and ineffective carrier sensing scenarios. Finally, an example illustrates the applications of this model.

\subsubsection{Goodput Distribution Model For Saturated Traffic Demand in Effective Carrier Sensing Scenarios}

Simulation results show that the disk-graph model can predict goodput pattern accurately in effective carrier sensing scenarios with saturated traffic demand (see Appendix A). Hence, Def. 1 establishes the goodput defined by typical disk-graph models in effective carrier sensing scenarios where all nodes are within each other's carrier sensing range.

Definition 1. The goodput in effective carrier sensing scenarios with saturated traffic: Assume that the channel capacity shared among the links in E has normalised as capacity 1 with respect to the maximum net bandwidth. The goodput $G(i)$ of a tagged link $i$ is defined as the ratio between goodput and maximum net bandwidth.

$$
G(i)=\frac{1}{N} \quad, \quad \text { when } \quad D \leq R_{c S}
$$

whereby $R_{c s}$ is the carrier sensing range, $D$ is the Cartesian distance between two border links, and $N$ is the number of links in the $E$.

The proof is given in Appendix B.1 and is immediate by Def. 1 and induction on the number of links in an IEEE 802.11 WBN.

\subsubsection{Goodput Distribution Model For Saturated Traffic Demand in Ineffective Carrier Sensing Scenarios}

For ineffective carrier sensing scenarios where not all nodes are within each other's carrier sensing range, the theorem is defined relating good- 
put with the notions of independent set and conflict set of a given link $i$. The theorem 1 expresses the goodput for each link $i$ as a function of independent set and conflict set. The theorem 1 is based on the observation in Appendix A.3.

Theorem 1. Goodput of a tagged link $i$ in ineffective carrier sensing scenarios $\left(D>R_{C S}\right)$ is a function of IS $(i)$ and $\gamma(i)$.

Definition 2. Independent set: Let $E$ denote the complete set of links in an IEEE 802.11 WBN. For a tagged link $i$ in $E$, the independent set,

$$
I S(i)=\left\{l \in E \backslash\{i\} \mid d_{s, s}>R_{c s} \text { and } d_{s, r}>R_{c s}\right\} .
$$

whereby $d_{s, s}$ is the distance between two senders in link $l$ and link $i, d_{s, r}$ is the distance between the sender in link $l$ and the receiver in link $i, R_{c s}$ is the carrier sensing range.

Definition 3. Conflict set: The conflict set of a tagged link $i$,

$$
\gamma(i)=\left\{l \in E \backslash\{i\} \mid d_{s, s} \leq R_{c s} \text { or } d_{s, s}>R_{c s}, d_{s, r} \leq R_{c s}\right\} .
$$

whereby $d_{s, s}$ is the distance between two senders in link $l$ and link $i, d_{s, r}$ is the distance between the sender in link $l$ and the receiver in link $i, R_{c s}$ is the carrier sensing range and it is clear that IS $(i) \cup \gamma(i) \cup i=E$.

To derive the goodput model, the border-link sets $\left(G C S_{L B}\right.$ and $\left.G C S_{R B}\right)$, the dominant border-link sets $G C S^{\prime}{ }_{L B}$ and $G C S^{\prime}{ }_{R B}$, and the middle-link set $\left(G C S_{M L}\right)$ are defined as follows based on our previous studies [116, 117].

Definition 4. Border-link sets $G C S_{L B}$ and $G C S_{R B}$ :

Let $E$ denote the complete set of links in an IEEE 802.11 WBN. For E, the left border-link set $G C S_{L B}$ and right border-link set $G C S_{R B}$,

$$
\begin{aligned}
& G_{C S} S_{L B}=\left\{l \in E \mid d_{l, R B}>R_{c s}\right\}, \\
& G_{C S} S_{R B}=\left\{l \in E \mid d_{l, L B}>R_{c S}\right\},
\end{aligned}
$$


whereby $d_{l, L B}$ is the distance between a link $l$ and the left border link, $d_{l, R B}$ is the distance between a link $l$ and the right border link, and $R_{c s}$ is the carrier sensing range. Note that the assumed topology will be a linear uniform and symmetric topology. The left and right border links are selected corresponding to the largest dimensions of the bounding box of all nodes in a given topology.

Definition 5. Middle-link set $G C S_{M L}$ : Let E denote the complete set of links in an IEEE 802.11 WBN. For E, the middle link set $G C S_{M L}$,

$$
G C S_{M L}=\left\{l \in E \backslash l \in G C S_{L B} \text { or } G C S_{R B}\right\},
$$

Definition 6. Dominant Border-link sets $G C S^{\prime}{ }_{L B}$ and $G C S^{\prime}{ }_{R B}$

Let $E$ denote the complete set of links in an IEEE 802.11 WBN. For E, the left dominant border-link set $\mathrm{GCS}{ }_{L B}$ and right dominant border-link set $\mathrm{GCS}^{\prime}{ }_{\mathrm{RB}}$,

$$
\begin{aligned}
& G_{C S}{ }_{L B}=\left\{l \in G C S_{L B} \mid d_{l, i}>R_{c S}\left\{i \in G C S_{R B}\right\}\right\}, \\
& G_{C S}^{\prime}{ }_{R B}=\left\{l \in G C S_{R B} \mid d_{l, i}>R_{C S}\left\{i \in G C S_{L B}\right\}\right\} \text {, }
\end{aligned}
$$

whereby $d_{l, i}$ is the distance between sender nodes in link $l$ and link $i$, and $R_{c s}$ is the carrier sensing range.

The above global border-link sets and middle-link set are used to identify the occurrence of flow starvation in a given IEEE 802.11 WBN. In an ineffective carrier sensing scenario, the links in the border-link sets have fewer conflicting links than those links in the middle-link set. Therefore, border effect brings potential risk of flow starvation to the links in the middle-link set. Some links in the border-link sets have the priority to occupy the spatial resource that influences the occurrence of border effect, which are selected to be the dominant border-link sets.

The topology shown in Figure 3.1 illustrates how to select these borderlink sets and middle-link set. Based on the Def. 4.4, Def 3.5, and Def. 3.6, the border-link sets and middle-link set are selected and shown in Figure 3.1. Two red circles represent the carrier sensing range from two border links $L 1$ and $L 9$. First, the left border-link set is selected as $G C S_{L B}=$ 


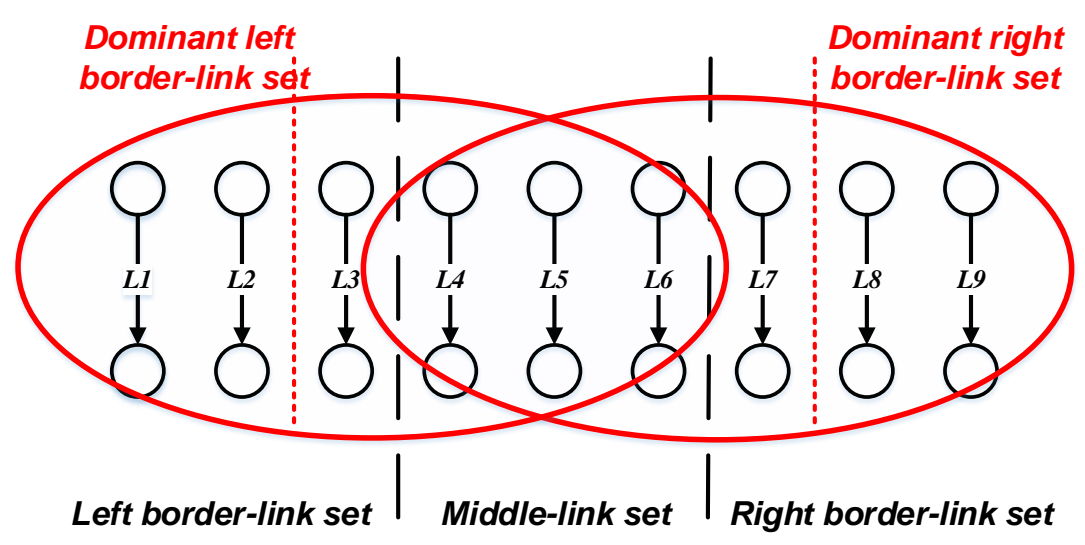

Figure 3.1: An example of border-link sets, dominant border-link sets, and middle-link set

$\{L 1, L 2, L 3\}$ and the right border-link set is selected as $G C S_{R B}=\{L 7, L 8, L 9\}$. Middle-link set is $G C S_{M L}=\{L 4, L 5, L 6\}$. The distance between link $L 3$ and $L 7$ is less than the carrier sensing range. Then, the dominant borderlink sets are selected as $G_{C S^{\prime}}{ }_{L B}=\{L 1, L 2\}$ and $G C S^{\prime}{ }_{R B}=\{L 8, L 9\}$.

Next, the minimum global conflict clique $\gamma^{G M C}$ is defined to calculate the spatial capacity in an IEEE 802.11 WBN. Different from the effective carrier sensing scenario without spatial reuse, spatial reuse exists in an ineffective carrier sensing scenario. Because the border links are out of each other's carrier sensing range and cannot sense each other, these links can transmit at the same time using an identical channel. Therefore, from the perspective of the whole network, the spatial capacity may exceed 1 . The minimum global conflict clique $\gamma^{G M C}$ is used to estimate the spatial capacity from the global view of a network.

For a graph $E$, a minimum global conflict clique is defined as a set of conflict sets $\gamma(i)$ that contains all the links in $E$ following the constraint in Def.7.

Definition 7. Minimum global conflict clique $\gamma^{G M C}$ : For a network E,

$$
\gamma^{G M C}:\{i, i \in E, j \in E: I S(i) \cap I S(j)=\varnothing\}
$$


whereby IS $(i)$ is the independent set of link $i$.

In the topolopgy in Figure 3.1, a minimum global conflict clique is $\{L 1, L 9\}$. The conflict sets of links $L 1$ and $L 9$ are $\gamma(L 1) \cup \gamma(L 9)=E$ and the corresponding independent sets are $I S(L 1) \cap I S(L 9)=\varnothing$. Then in this topology, the maximum spatial capacity will be $\left|\gamma^{G M C}\right|=|\{L 1, L 9\}|=2$.

The goodput distribution model is proposed with a pessimistic goodput $G_{P}(i)$ and an optimistic goodput $G_{O}(i)$ in ineffective carrier sensing scenarios. This idea arises from two distinct situations. The first situation is that the conflicting links of a given link are within each other's $R_{c s}$ (e.g. all conflicting links of $L 1$ are within each other's $R_{C S}$ in Figure 3.1). In this case, the sum of goodput of a tagged link $i$ and all conflicting links will be $1, G(i)+\sum_{j \in \gamma(i)} G(j)=1$.

The second situation is that the conflicting links of a given link are not all within each other's $R_{c s}$ (e.g. $L 5$ 's conflict set is $\{1,2,3,4,6,7,8,9\}$ but $L 1$ and $L 9$ are out of each other's $R_{C S}$ ). Unlike the previous case, the sum of goodput of $L 5$ and its conflicting links $L 1-L 9$ may exceed the total channel capacity 1 , i.e. $1<G(i)+\sum_{j \in \gamma(i)} G(j) \leq\left|\gamma^{G M C}\right|$.

To address the two distinct situations, the pessimistic and optimistic estimate of the goodput are proposed for a given link $i$. For the pessimistic estimate of goodput for a tagged link, the lower bound of the achievable goodput is restricted such that $G_{P}(i)+\sum_{j \in \gamma(i)} G_{P}(j)=1$. This goodput is regarded as the pessimistic goodput estimate.

In terms of the optimistic estimate of the goodput, the goodput is calculated by using the conflict set of the border link for the links within the conflict set of the border link. The border link attains the highest goodput and has the fewest number of links in its conflict set. Hence, using the conflict set with the least number of links, the upper bound of the achievable goodput is restricted such that $G_{O}(i)+\sum_{j \in \gamma(B)} G_{O}(j)=1$. This goodput is regarded as the optimistic estimate of the goodput.

Next, the pessimistic and optimistic goodput $G_{P}(i)$ and $G_{O}(i)$ of a link $i$ in the ineffective carrier sensing scenarios are defined as follows. 
Definition 8. Pessimistic goodput for ineffective carrier sensing scenarios with saturated traffic $G_{P}(i)$ : The pessimistic goodput is defined as the ratio between goodput and maximum net bandwidth,

$$
G_{P}(i)=\left\{\begin{array}{cc}
0 & \chi(i) \times\left(1-\left|G C S_{M L}\right| \times \alpha \times \frac{\left|\gamma^{G M C}\right|}{N}\right) \\
\frac{\chi(i)+\sum_{j \in \gamma(i)} \chi(j)}{N}, & \text { otherwise }
\end{array}\right.
$$

subject to $G_{P}(i)+\sum_{j \in \gamma(i)} G_{P}(j)=1$, where $\chi(i)$ is the number of links in a given IS $(i), \gamma(i)$ is the conflict set of link $i,\left|G C S_{M L}\right|$ is the cardinality of the global middle link set $G C S_{M L},\left|\gamma^{G M C}\right|$ is the cardinality of the minimum global clique $\gamma^{G M C}, N$ is the number of links in $E$ and $\alpha$ is the starvation factor.

Definition 9. Optimistic goodput for ineffective carrier sensing scenarios with saturated traffic $G_{O}(i)$ : The optimistic goodput is defined as the ratio between goodput and maximum net bandwidth,

$$
G_{O}(i)=\left\{\begin{array}{cc}
\alpha \times \frac{\left|\gamma^{G M C}\right|}{N} & , \quad i \in G C S_{M L}, \\
\frac{\chi(i)}{\chi(i)+\sum_{j \in \gamma(B)} \chi(j)} \quad, & \text { otherwise, }
\end{array}\right.
$$

subject to $G_{O}(B)+\sum_{j \in \gamma(B)} G_{O}(j)=1$, where $\gamma(B)$ is the conflict set of the border link $B$ in which the link $i$ is and $\chi(i)$ is the number of links in a given $I S(i),\left|\gamma^{G M C}\right|$ is the cardinality of the minimum global clique $\gamma^{G M C}, N$ is the number of links in $E$ and $\alpha$ is the starvation factor.

Proofs of the pessimistic and optimistic goodput are by definition and induction on the number of links in an IEEE 802.11 WBN and is given in Appendix B.2. Note the starvation factor $\alpha$ ranging from 0.0 to 0.2 in the pessimistic and optimistic goodput equations is the empirical value from simulation results. 


\subsubsection{Example: Using the Mmodel with Saturated Traffic Demand}

For saturated traffic demand, an example demonstrates the utility of the proposed goodput model shown in Figure 3.2. Figure 3.2 shows a linear, uniformly spaced and symmetric topology that the network size $D$ is $800 \mathrm{~m}$ and the inter-link interval $d$ is $100 \mathrm{~m}$. The network $E$ for this scenario is $E=\{L 1, L 2, \ldots, L 9\}$. The transmitter-receiver separation of all links $D_{t r}$ is $50 \mathrm{~m}$. The propagation model is the two-ray propagation model and carrier sensing range $R_{c S}$ is $515 \mathrm{~m}$. The traffic demand of each link is configured as $24 \mathrm{Mbps}$ (for saturating the wireless link).

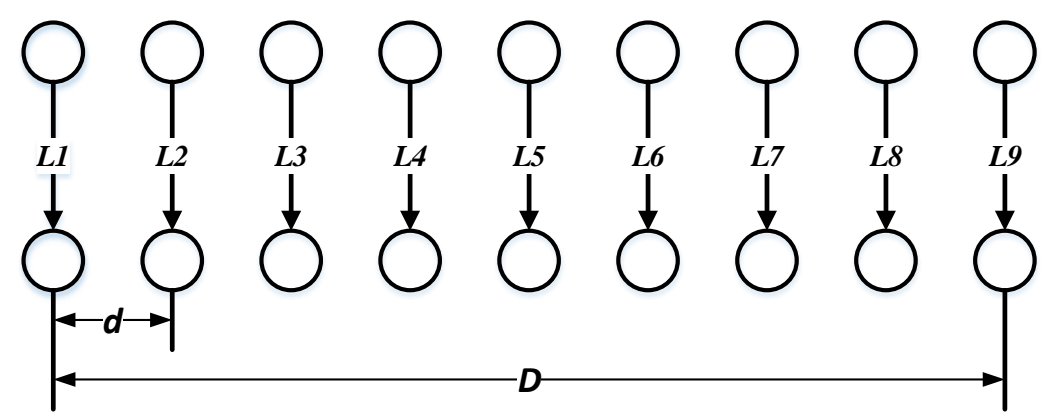

Figure 3.2: Nine Links deployed in a $d=100 m, 800 \times 50 m^{2}$ IEEE 802.11 WBN

First, the independent set $I S(i)$ and conflict set $\gamma(i)$ are calculated for all links based on the topology information and the given $R_{c S}$ (see Table 3.2). According to the results tabulated in Table 3.2. links $L 4$ to $L 6$ are within the $R_{c S}$ of both border links $L 1$ and $L 9$ and these middle links have no independent links as $\{\varnothing\}$. Links 4 to 6 are predicted to achieve "zero" goodput for pessimistic goodput (and hence are called starving links). We calculate $\gamma^{G M C}=\left\{L_{1}, L 9\right\}$ and $\left|\gamma^{G M C}\right|=2$. For starving links (assume $\alpha=0.2)$, their optimistic goodput is calculated as $\alpha \times \frac{\left|\gamma^{G M C}\right|}{N}=0.044$.

For the non-starving links, the weight is assigned to each link based on definition of $\chi(i)$. For example, link $L 3$ 's independent set has only one link $L 9$, thus link L3's weight is assigned as 1 , and same for link $L 2$ with 
Table 3.2: Independent sets, conflict sets, and goodput of individual links

\begin{tabular}{cccccc}
\hline Link $i$ & $I S(i)$ & $\gamma(i)$ & $\chi(i)$ & $G_{P}(i)$ & $G_{O}(i)$ \\
\hline 1 & $\{7,8,9\}$ & $\{2,3,4,5,6\}$ & 3 & 0.433 & 0.500 \\
2 & $\{8,9\}$ & $\{1,3,4,5,6,7\}$ & 2 & 0.248 & 0.333 \\
3 & $\{9\}$ & $\{1,2,4,5,6,7,8\}$ & 1 & 0.096 & 0.167 \\
4 & $\{\varnothing\}$ & $\{1,2,3,5,6,7,8,9\}$ & 0 & 0.000 & 0.044 \\
5 & $\{\varnothing\}$ & $\{1,2,3,4,6,7,8,9\}$ & 0 & 0.000 & 0.044 \\
6 & $\{\varnothing\}$ & $\{1,2,3,4,5,7,8,9\}$ & 0 & 0.000 & 0.044 \\
7 & $\{1\}$ & $\{2,3,4,5,6,8,9\}$ & 1 & 0.096 & 0.167 \\
8 & $\{1,2\}$ & $\{3,4,5,6,7,9\}$ & 2 & 0.248 & 0.333 \\
9 & $\{1,2,3\}$ & $\{3,4,5,6,7,8\}$ & 3 & 0.433 & 0.500 \\
\hline
\end{tabular}

weight 2. Using Def. 8 and Def. 9, the pessimistic and optimistic goodput $G_{P}(i)$ and $G_{O}(i)$ are calculated for this example listed in Table 3.2 .

\subsubsection{A Goodput Distribution Model For Unsaturated Traf- fic Demand}

This subsection derives the goodput model with unsaturated traffic demand. With unsaturated traffic demands, whether starvation would happen depends on the traffic demands among border links (see the discussion in Appendix A). When unsaturated traffic demands of border links do not exceed channel capacity, middle links may not get starved. The assumptions for ineffective carrier sensing scenarios with unsaturated traffic demand are the same as those in Section 3.1.1 except the Assumption 4. For unsaturated traffic demand, border effect may not exist even if the distance between two border links exceeds the carrier sensing range. The occurrence of the border effect depends on the traffic demand from the border link sets.

The next subsections define the goodput model for effective and ineffective carrier sensing scenarios followed by an example. 


\subsubsection{Goodput Distribution Model For Unsaturated Traffic Demand in Effective Carrier Sensing Scenarios}

Simulation results show that the disk-graph model can predict the goodput pattern accurately in effective carrier sensing scenarios with unsaturated traffic demand (see Appendix A). Hence, Def. 10 establishes the goodput defined by typical disk-graph models in effective carrier sensing scenarios where all nodes are within each other's carrier sensing range.

Definition 10. Goodput distribution for effective carrier sensing scenarios with unsaturated traffic: The goodput $G(i)$ of a tagged link $i$ is defined as the ratio between goodput and maximum net bandwidth.

$$
G(i)=\left\{\begin{array}{cll}
\frac{1}{N} & \text {, when } & \sum_{i \in E} f(i)>1 \\
f(i) & \text {, when } & \sum_{i \in E} f(i) \leq 1
\end{array}\right.
$$

whereby $N$ is the number of links in the $E$, and $f(i)$ denotes the normalised traffic demand of a link $i$ with respect to the maximum net capacity. Note that the maximum net capacity in this thesis refers to goodput defined in Section 2.1.

The proof for the goodput Def. 10 is given in Appendix B.3.

\subsubsection{Goodput Distribution Model For Unsaturated Traffic Demand in Ineffective Carrier Sensing Scenarios}

The expected goodput pattern from the disk-graph model is observed differently from simulation results in ineffective sensing scenarios with unsaturated traffic demand (see Appendix A. Complex interactions between border links and middle links exist in ineffective carrier sensing scenario. Therefore, correction factors need to be introduced to this scenario with a new criterion.

For ineffective carrier sensing scenario, two criteria, A and B, are defined to refine the possible combinations of unsaturated traffic demands. 
First, criterion A is the sum of traffic demands of the links in the dominant border-link sets $G C S^{\prime}{ }_{L B}$ and $G C S^{\prime}{ }_{R B}$, and this is expressed as:

$$
\sum_{j \in G C S^{\prime}{ }_{L B} \cap G C S^{\prime}{ }_{R B}} f(j) \geq 1
$$

Criterion A is to evaluate whether border effect occurs. If the criterion A holds, border effect potentially exists and the border links will occupy the channel and middle links will have very little chances to transmit packets that lead to flow starvation. If not, border effect and flow starvation will not exist.

Criterion $B$ is the sum of traffic demands of the links in the left or right border links with the links in its conflict set $\gamma(B)$, and this is expressed as:

$$
\sum_{j \in B \cap \gamma(B)} f(j) \geq 1
$$

Criterion B is to evaluate whether the channel capacity is sufficient for the border link and the links in its conflict set. If criterion B holds, the channel capacity is not sufficient and the border link will have priority to occupy the channel over its conflicting links. If not, the border link and its conflicting links will share the channel capacity fairly.

We list four possible combinations of unsaturated traffic demands with two criteria A and B in Table 3.3. For each condition, different equations are used to calculate goodput distribution. For example, if the traffic demands among all links satisfy condition \#2, Def. 11 and Def. 12 will be used to calculate the goodput distribution.

Definition 11. Pessimistic goodput for conditions \#1 and \#2 with unsaturated traffic $G_{P}(i)$ : The pessimistic goodput of a tagged link $i$ is defined as the ratio between goodput and maximum net bandwidth.

$$
G_{P}(i)=\left\{\begin{array}{cc}
0 & , i \in G C S_{M L}, \\
\min \left(f(i), \frac{\chi(i) \times\left(1-\alpha \times\left|G C S_{M L}\right| \times \frac{\left|\gamma^{G M C}\right|}{N}\right)}{\chi(i)+\sum_{j \in \gamma(i)} \chi(j)}\right) & , \text { otherwise }
\end{array}\right.
$$


Table 3.3: Four unsaturated traffic demands in ineffective carrier sensing scenarios

\begin{tabular}{l|c|c|c|c|}
\hline Condition & Criterion A & Criterion B & \multicolumn{2}{|c}{ Predicted goodput } \\
\hline Condition \#1 & True & True & Def. 11 & and Def. 12 \\
\hline Condition \#2 & True & False & Def. 11 & and Def. 12 \\
\hline Condition \#3 & False & True & Def. 13 & and Def. 14 \\
\hline Condition \#4 & False & False & \multicolumn{2}{|c|}{ Def. 15 } \\
\hline
\end{tabular}

whereby $\left|G C S_{M L}\right|$ is the cardinality of the middle-link set $G C S_{M L}, f(i)$ is the normalised traffic demand of a link $i$ with respect to the maximum net capacity, $\chi(i)$ denotes the number of links in a given IS $(i)$ and $\gamma(i)$ is the conflict set of a tagged link $i,\left|\gamma^{G M C}\right|$ is the cardinality of the minimum global clique $\gamma^{G M C}, \alpha$ is the starvation factor and $N$ is the number of links in $E$.

Definition 12. Optimistic goodput for conditions \#1 and \#2 with unsaturated traffic $G_{O}(i)$ : The optimistic goodput of a tagged link $i$ is defined as the ratio between goodput and maximum net bandwidth.

$$
G_{O}(i)=\left\{\begin{array}{cc}
\alpha \times \frac{\left|\gamma^{G M C}\right|}{N} & , i \in \operatorname{GCS}_{M L} \\
\min \left(f(i), \frac{\chi(i)}{\chi(i)+\sum_{j \in \gamma(B)} \chi(j)}\right) & , \text { otherwise }
\end{array}\right.
$$

whereby GCS $_{M L}$ is middle-link set, $\chi(i)$ denotes the number of links in a given IS $(i)$ and $\gamma(B)$ is the conflict set of the border link LB or $R B, f(i)$ is the normalised traffic demand of a link $i$ with respect to the maximum net capacity, $\left|\gamma^{G M C}\right|$ is the cardinality of the minimum global clique $\gamma^{G M C}, \alpha$ is the starvation factor and $N$ is the number of links in $E$.

Definition 13. Pessimistic goodput for condition \#3 with unsaturated traffic $G_{P}(i)$ : The pessimistic goodput of a tagged link $i$ is defined as the ratio between goodput and maximum net bandwidth. 
$G_{P}(i)=\left\{\begin{array}{cc}0.9 \times \min \left(f(i), \frac{1-\sum_{j \in \gamma(B)} f(j)}{\left|G S B_{M L}\right|}\right) & , i \in G C S_{M L} \\ 0.9 \times f(i) & , i \in G S^{\prime}{ }_{L B} \text { or } G_{C S^{\prime}}{ }_{R B}, \\ 0.9 \times \min \left(f(i), \frac{\chi(i) \times\left(1-\sum_{j \in G C S_{B}{ }_{B}} f(j)\right)}{\chi(i)+\sum_{l \in \gamma(i) a n d \notin G C S_{B}{ }_{B}} \chi(j)}\right) & , \text { otherwise, }\end{array}\right.$

whereby $G_{C S}{ }_{L B}$ and $G_{C S}{ }_{R B}$ are border-link sets, $\chi(i)$ denotes the number of links in a given IS $(i), \gamma(i)$ is the conflict set of a link $i,\left|G S B_{M L}\right|$ is the cardinality of the set $G C S_{M L}$, and $f(i)$ denotes the traffic demand of a link $i$ with respect to the maximum net capacity. Note the factor " 0.9 " in the pessimistic goodput equations is the empirical value from simulation results.

Definition 14. Optimistic goodput for condition \#3 with unsaturated traffic $G_{O}(i)$ : The optimistic goodput of a tagged link $i$ is defined as the ratio between goodput and maximum net bandwidth.

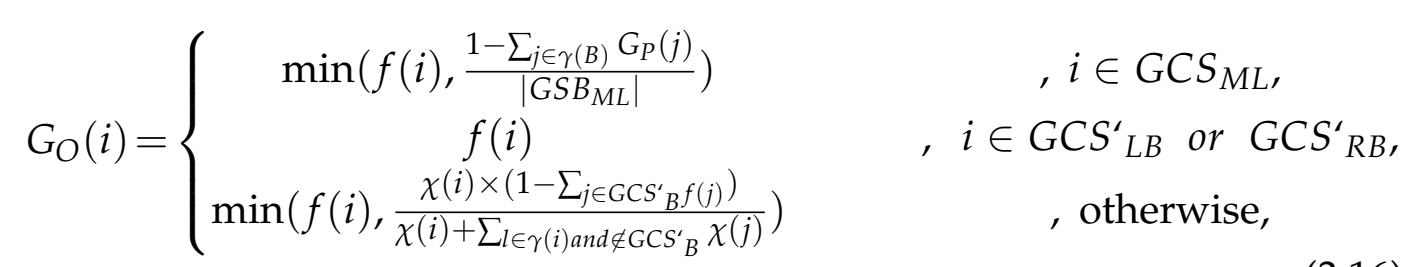

whereby $\left|G S B_{M L}\right|$ is the cardinality of the middle-link set $G C S_{M L}, \chi(i)$ is the number of links in $I S(i), f(i)$ denotes the traffic demand of a link $i$ with respect to the maximum net capacity, and $\gamma(B)$ is the conflict set of the left or right border link.

Definition 15. Pessimistic and optimistic goodput for condition \#4 with unsaturated traffic: The goodput of a tagged link $i$ is defined as the ratio between goodput and maximum net bandwidth.

$$
G_{P}(i)=G_{O}(i)=f(i),
$$

whereby $f(i)$ denotes the traffic demand of a link $i$ with respect to the maximum net capacity. 


\subsubsection{Example: Using the Model with Unsaturated Traffic Demand}

Here, an example is used to explain how to calculate goodput distribution with unsaturated traffic demand with the topology in Figure 3.2. Figure 3.2 shows a linear, uniformly spaced and symmetric topology that the network size $D$ is $800 \mathrm{~m}$ and the inter-link interval $d$ is $100 \mathrm{~m}$. The network $E$ for this scenario is $E=\{L 1, L 2, \ldots, L 9\}$.

Table 3.4: Goodput estimation of individual links

\begin{tabular}{c|ccc|ccc}
\hline Link $i$ & $f(i)$ & $G_{P}(i)$ & $G_{O}(i)$ & $f(i)$ & $G_{P}(i)$ & $G_{O}(i)$ \\
\hline 1 & 0.8 & 0.433 & 0.500 & 0.1 & 0.100 & 0.100 \\
2 & 0.8 & 0.248 & 0.333 & 0.1 & 0.100 & 0.100 \\
3 & 0.8 & 0.096 & 0.167 & 0.1 & 0.100 & 0.100 \\
4 & 0.8 & 0.000 & 0.044 & 0.1 & 0.100 & 0.100 \\
5 & 0.8 & 0.000 & 0.044 & 0.1 & 0.100 & 0.100 \\
6 & 0.8 & 0.000 & 0.044 & 0.1 & 0.100 & 0.100 \\
7 & 0.8 & 0.096 & 0.167 & 0.1 & 0.100 & 0.100 \\
8 & 0.8 & 0.248 & 0.333 & 0.1 & 0.100 & 0.100 \\
9 & 0.8 & 0.433 & 0.500 & 0.1 & 0.100 & 0.100 \\
\hline
\end{tabular}

In Table 3.4, two traffic demands $f(i)$ for all links are selected as 0.8 and 0.1 (normalised traffic demand with respect to the maximum net capacity). For $f(i)=0.8$, the traffic demands of all links satisfy the condition \#1 in Table 3.3. The border effect exists and the channel capacity is not sufficient to satisfy the traffic demand for all the links. Hence, Def. 11 and Def. 12 are selected to calculate $G_{P}(i)$ and $G_{O}(i)$ where $\alpha$ is assumed as 0.2.

For $f(i)=0.1$, the traffic demands of all links satisfy the condition \#4 in Table 3.3. The border effect does not exist and the channel capacity is sufficient to satisfy the traffic demand for all the links. Def. 15 is selected to calculate $G_{P}(i)$ and $G_{O}(i)$. 


\subsubsection{Summary}

This section introduces the derivation of a unified goodput distribution model covering effective and ineffective carrier sensing scenarios. This unified goodput model provides link-level goodput distribution with the given inputs: (i) topology, (ii) carrier sensing range, (iii) traffic demand. The next sections will discuss the validation of the proposed goodput model through simulations using different propagation models.

\subsection{Simulation Validation with Two-ray Propa- gation Model}

This section validates the accuracy of the proposed goodput model through simulation with the two-ray ground model [66]. All the simulations are conducted in QualNet 5.2. Table 3.5 lists the main configuration parameters. Note that in this thesis, only physical carrier sensing (PCS) is used without using virtual carrier sensing.

The theoretical maximum transmission range $D_{t r}^{\max }$ in this simulation is approximately $207 \mathrm{~m}$. This value is calculated by QualNet's radio range utility with the simulation scenario as input. Moreover, physical carrier sensing range $R_{c s}$ is defined by a triplet consisting of (i) the minimum receiver sensitivity of $-78 \mathrm{dBm}$, (ii) maximum transmission power of $18 \mathrm{dBm}$ (based on Alcatel Lucent WaveLAN card), and (iii) the two-ray propagation model, which yields the distance of $515 \mathrm{~m}$.

To match the assumptions in Section 3.1, the simulation was conducted following: (i) all nodes are configured with identical parameters and choose constant bit rate (CBR) unicast transmission as the application with saturated and unsaturated traffic demands(see Table 3.5); (ii) all nodes are configured with one radio interface and the same channel. Node placement is based on a linear topology (see Figure 3.3); (iii) transmitter-receiver separation is chosen as $50 \mathrm{~m}$ to guarantee collision-free transmissions (based 
on the findings from [156]).

Table 3.5: Simulation configuration parameters

\begin{tabular}{l|l}
\hline Parameter Name & Value \\
\hline Transmission Power & $18 \mathrm{dBm}$ \\
\hline Receiver Sensitivity & $-78 \mathrm{dBm}$ \\
\hline Path Loss Model & Two-Ray \\
\hline Shadowing and Fading Model & None \\
\hline Physical Layer & IEEE $802.11 \mathrm{a}$ \\
\hline Data Rate & $24 \mathrm{Mbps}$ \\
\hline MAC Layer & PCS \\
\hline Routing & Static Routing \\
\hline Transportation Layer & UDP \\
\hline Packet Size & 1500 Bytes \\
\hline Inter-packet Interval for Saturated Traffic & $0.5 \mathrm{~ms}$ \\
\hline Inter-packet Interval for Unsaturated Traffic & $0.5-3.45 \mathrm{~ms}$ \\
\hline
\end{tabular}

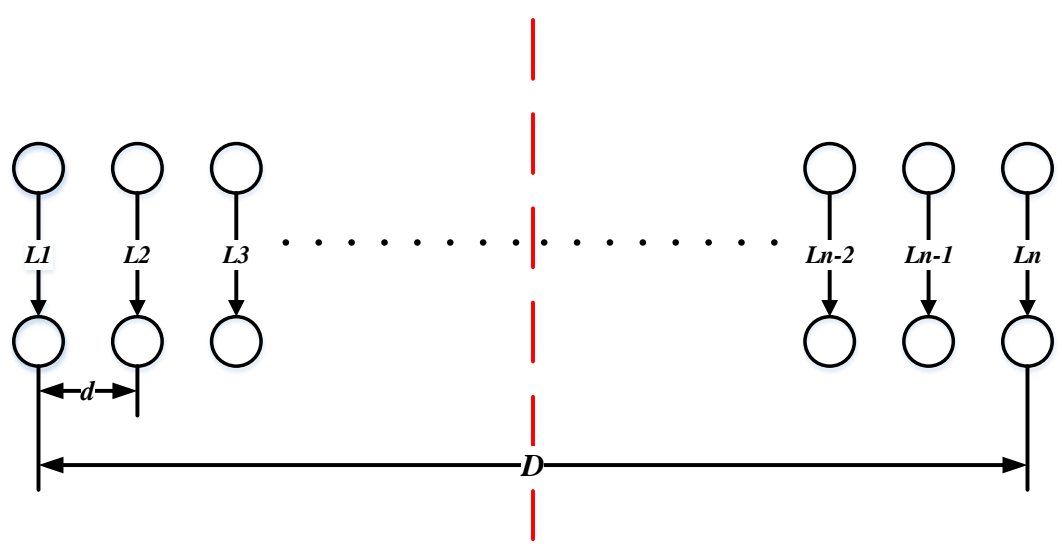

Figure 3.3: The topology used in simulations

The topologies will cover effective and ineffective carrier sensing scenarios ranging from $200 \times 50 \mathrm{~m}^{2}$ to $800 \times 50 \mathrm{~m}^{2}$ (see Figure 3.3). When the border distance $D$ is less than the carrier sensing range, the scenario 
is regarded as an effective carrier sensing scenario, while when the border distance $D$ is greater than the carrier sensing range, the scenario represents the ineffective carrier sensing scenario.

To validate the accuracy of our analytical model, the goodput model will be used to predict goodput distribution and compared with the simulation mean value. If the mean value of simulation result falls between the prediction of the proposed model, the goodput model predicts the goodput accurately. If not, the error ratio ER is calculated as follows.

Definition 16. Error Ratio ER

Let $E$ denote the set of links in an IEEE 802.11 WBN,

$$
E R=\left|\frac{\left(G_{s}(i)-G_{\text {Mclose }}(i)\right)}{G_{s}}\right|,
$$

where $G_{s}(i)$ is the mean goodput of a link i from simulation, $G_{M c l o s e}(i)$ is the closest goodput prediction from the goodput model.

Part of the results in the following subsections appears in our previous work [114, 116]. Simulation results shown in this section are averages from 100 randomly seeded simulation runs. All averages of simulation results shown are reported with confidence interval of $95 \%$ with the range of $[2.746,34.359] \mathrm{kbps}$ under the assumption that the averages are normally distributed. Note that the above range of confidence interval is calculated among all the simulation results that include the examples listed in this chapter.

The next subsections categorise the simulation results into two parts, saturated and unsaturated traffic demands.

\subsubsection{Saturated Traffic Demand}

For saturated traffic demand, the sender of each link in the simulation attempts to transmit packets at the maximal data rate $24 \mathrm{Mbps}$. Due to space, two simulation results are listed as examples for effective and ineffective carrier sensing scenarios. For the effective carrier sensing scenario, 
the topology in Figure 3.3 is selected with the border distance $D$ as $200 \mathrm{~m}$, where all nodes are within each other's $R_{c s}$. This example is selected based on the wind turbines connected to the smart grid in Brooklyn, Wellington, New Zealand. Each wind turbine is installed with a wireless router to exchange data, such as generated energy and error logs (see Figure 3.4).

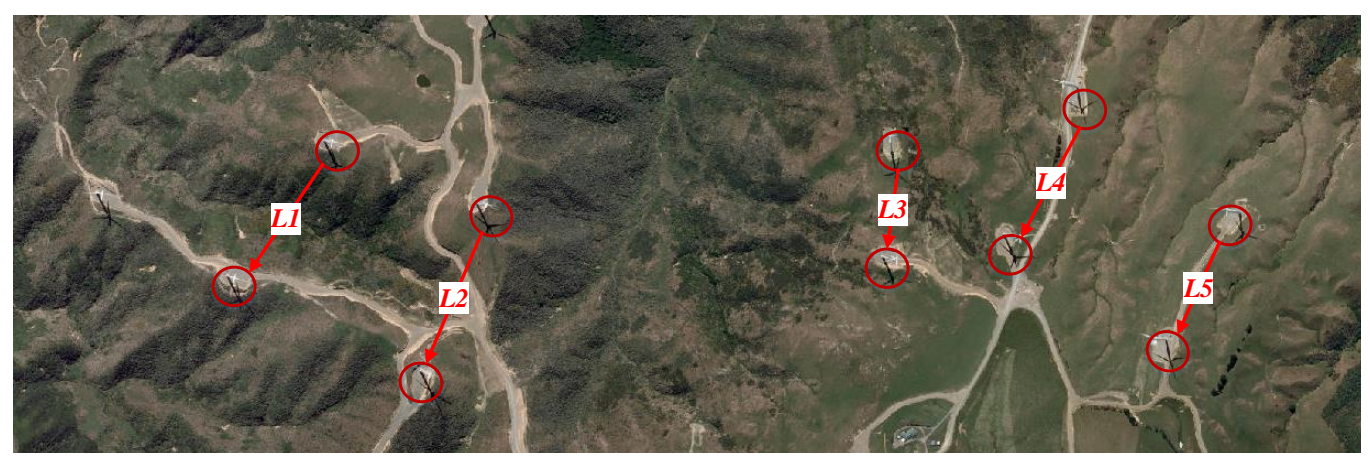

Figure 3.4: An example of the IEEE 802.11 WBN in a smart grid system representing two-ray ground propagation model

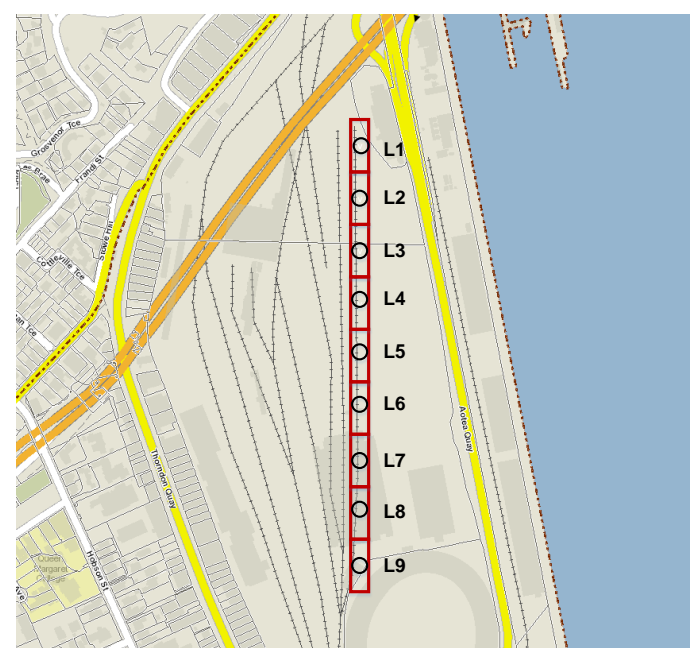

Figure 3.5: An example of the IEEE 802.11 WBN in an intelligent transportation system representing two-ray ground propagation model 
For the ineffective carrier sensing scenarios, the topology in Figure 3.3 is selected with the border distance $D$ as $800 \mathrm{~m}$, where two border links are out of each other's carrier sensing range. This example is to model the intelligent transportation system (ITS) along the rail track between Wellington and Petone in New Zealand (see Figure 3.5). The ITS collects data on train load and train delays, and feeds it into a data portal for storage and analysis.

In Tables 3.6 to 3.7, the first column refers to the link index in the topology (see Figure 3.3). Goodput $G_{S}(i)$ refers to the simulation mean value of goodput. Goodput $G(i)$, pessimistic goodput $G_{P}(i)$ and optimistic goodput $G_{O}(i)$ are the predictions from the proposed goodput model. ER denotes the difference between $G_{S}(i)$ and the closest goodput prediction from the new goodput model (see Def. 16).

Table 3.6: Goodput of five links in a $200 \times 50 \mathrm{~m}^{2}$ topology with two-ray propagation model using saturated traffic

\begin{tabular}{cccc}
\hline Link $i$ & $G_{S}(i)$ & $G(i)$ & $E R$ \\
\hline 1 & $0.230^{*}$ & 0.200 & 0.130 \\
2 & 0.161 & 0.200 & - \\
3 & 0.152 & 0.200 & - \\
4 & 0.161 & 0.200 & - \\
5 & $0.231^{*}$ & 0.200 & 0.134 \\
\hline
\end{tabular}

In effective carrier sensing scenario with saturated traffic demand, the results in Table 3.6 show that different links achieve different goodput. We mark the simulation results that slightly exceed the prediction from the new model with $\left(^{*}\right)$. In the new goodput model, we assume that all links share the channel capacity, which matches with the expectation from disk-graph model. However, in practice there is a small variation in goodput among all the links. In this case, border links achieve higher goodput than the middle links. It implies that in effective carrier sensing scenarios, 
the randomised binary exponential back-off scheme used in IEEE 802.11 carrier sensing may result in a slight difference among the goodput of neighbouring links rather than achieving extreme equal goodput for each link [10]. The overall difference ratio is below $13.5 \%$.

For the ineffective scenario, the topology is chosen with a fixed border distance, $D=800 \mathrm{~m}$ to ensure that the two border links are always out of each other's $R_{c s}$ and border effect should occur. Table 3.7 displays the comparison between the results from simulations and those from the new goodput model. The grey cells in these two tables denote the links identified as starving links.

Table 3.7: Goodput of nine links in a $800 \times 50 \mathrm{~m}^{2}$ topology with two-ray propagation model using saturated traffic

\begin{tabular}{ccccc}
\hline Link $i$ & $G_{S}(i)$ & $G_{P}(i)$ & $G_{O}(i)$ & $E R$ \\
\hline 1 & $0.502^{*}$ & 0.433 & 0.500 & 0.004 \\
2 & 0.309 & 0.248 & 0.333 & - \\
3 & 0.146 & 0.096 & 0.167 & - \\
4 & 0.009 & 0.000 & 0.040 & - \\
5 & 0.008 & 0.000 & 0.040 & - \\
6 & 0.009 & 0.000 & 0.040 & - \\
7 & 0.146 & 0.096 & 0.167 & - \\
8 & 0.309 & 0.248 & 0.333 & - \\
9 & $0.501^{*}$ & 0.433 & 0.500 & 0.002 \\
\hline
\end{tabular}

The data in Table 3.7 shows the new model predicts starving links correctly (the starvation factor $\alpha$ is selected as 0.2 ). For the non-starving links, all the simulation mean values fall between the range of the predictions from the new model. In ineffective carrier sensing scenarios with saturated traffic demands, the proposed goodput model provides accurate prediction of starvation and goodput. 


\subsubsection{Unsaturated Traffic Demand}

To validate the new model with unsaturated traffic demand, four unsaturated traffic demands are selected by using different inter-packet intervals. The traffic demands $f(i)$ at the application layer can be normalised as 0.8 , $0.6,0.4$, and 0.1 with respect to the maximum net capacity by using the configuration parameters in Table 3.5. Similar to the above subsection, two topologies are chosen to represent effective and ineffective carrier sensing scenarios.

Table 3.8: Goodput of five links in a $200 \times 50 \mathrm{~m}^{2}$ topology with two-ray propagation model using unsaturated traffic

\begin{tabular}{c|cccc|cccc}
\hline Link $i$ & $f(i)$ & $G_{S}(i)$ & $G(i)$ & $E R$ & $f(i)$ & $G_{S}(i)$ & $G(i)$ & $E R$ \\
\hline 1 & 0.8 & $0.231^{*}$ & 0.200 & 0.134 & 0.6 & $0.230^{*}$ & 0.200 & 0.130 \\
2 & 0.8 & 0.160 & 0.200 & - & 0.6 & 0.161 & 0.200 & - \\
3 & 0.8 & 0.153 & 0.200 & - & 0.6 & 0.152 & 0.200 & - \\
4 & 0.8 & 0.161 & 0.200 & - & 0.6 & 0.161 & 0.200 & - \\
5 & 0.8 & $0.230^{*}$ & 0.200 & 0.130 & 0.6 & $0.230^{*}$ & 0.200 & 0.130 \\
\hline
\end{tabular}

Table 3.9: Goodput of five links in a $200 \times 50 \mathrm{~m}^{2}$ topology with two-ray propagation model using unsaturated traffic-continued

\begin{tabular}{c|cccc|cccc}
\hline Link $i$ & $f(i)$ & $G_{S}(i)$ & $G(i)$ & $E R$ & $f(i)$ & $G_{S}(i)$ & $G(i)$ & $E R$ \\
\hline 1 & 0.4 & $0.230^{*}$ & 0.200 & 0.130 & 0.1 & 0.100 & 0.100 & - \\
2 & 0.4 & 0.161 & 0.200 & - & 0.1 & 0.100 & 0.100 & - \\
3 & 0.4 & 0.153 & 0.200 & - & 0.1 & 0.100 & 0.100 & - \\
4 & 0.4 & 0.160 & 0.200 & - & 0.1 & 0.100 & 0.100 & - \\
5 & 0.4 & $0.231^{*}$ & 0.200 & 0.134 & 0.1 & 0.100 & 0.100 & - \\
\hline
\end{tabular}

The results of the effective carrier sensing scenario $(D=200 \mathrm{~m})$ are listed in Tables 3.8 and 3.9 . The function $f(i)$ refers to the normalised 
traffic demand with respect to the maximum net capacity while goodput $G_{S}(i)$ and $G_{P}(i) / G_{O}(i)$ denote the simulation goodput mean values and the predicted goodput from the new model.

The results show the same goodput pattern as that in saturated traffic demand. Only in the case $f(i)=0.1$, all links achieve the same goodput. For other traffic demands, there is a small variation in goodput among all the links. Along the same lines of explanation in Section 3.2.1, in effective carrier sensing scenarios, the randomised binary exponential back-off scheme used in IEEE 802.11 carrier sensing may result in a slight difference among the goodput of neighbouring links rather than achieving extreme equal goodput for each link [10]. The overall error is below $13.5 \%$.

Table 3.10: Goodput of nine links in a $800 \times 50 \mathrm{~m}^{2}$ topology with two-ray propagation model using unsaturated traffic

\begin{tabular}{c|cccc|cccc}
\hline & \multicolumn{5}{|c|}{$f(i)=0.8$} & \multicolumn{4}{c}{$f(i)=0.6$} \\
Link $i$ & $G_{S}(i)$ & $G_{O}(i)$ & $G_{P}(i)$ & $E R$ & $G_{S}(i)$ & $G_{O}(i)$ & $G_{P}(i)$ & $E R$ \\
\hline 1 & 0.502 & 0.433 & 0.500 & 0.004 & 0.501 & 0.433 & 0.500 & 0.002 \\
2 & 0.301 & 0.248 & 0.333 & - & 0.310 & 0.248 & 0.333 & - \\
3 & 0.146 & 0.096 & 0.167 & - & 0.146 & 0.096 & 0.167 & - \\
4 & 0.009 & 0.00 & 0.044 & - & 0.009 & 0.00 & 0.044 & - \\
5 & 0.008 & 0.00 & 0.044 & - & 0.008 & 0.00 & 0.044 & - \\
6 & 0.009 & 0.00 & 0.044 & - & 0.009 & 0.00 & 0.044 & - \\
7 & 0.146 & 0.096 & 0.167 & - & 0.146 & 0.096 & 0.167 & - \\
8 & 0.309 & 0.248 & 0.333 & - & 0.309 & 0.248 & 0.333 & - \\
9 & 0.501 & 0.433 & 0.500 & 0.002 & 0.502 & 0.433 & 0.500 & 0.004 \\
\hline
\end{tabular}

Tables 3.10 and 3.11 list the results with unsaturated traffic demands in the ineffective carrier sensing scenarios where $D$ is selected as $800 \mathrm{~m}$ and $d$ as $100 \mathrm{~m}$. Results in Tables 3.10 and 3.11 show that most of the simulation mean values fall between the prediction range, proving the proposed model predicts starvation and goodput distribution accurately. The overall error is below $6 \%$. Overall, the proposed goodput model performs 
Table 3.11: Goodput of nine links in a $800 \times 50 \mathrm{~m}^{2}$ topology with two-ray propagation model using unsaturated traffic-continued

\begin{tabular}{c|cccc|cccc}
\hline & \multicolumn{5}{|c|}{$f(i)=0.4$} & \multicolumn{4}{c}{$f(i)=0.1$} \\
Link $i$ & $G_{S}(i)$ & $G_{P}(i)$ & $G_{O}(i)$ & $E R$ & $G_{S}(i)$ & $G_{P}(i)$ & $G_{O}(i)$ & $E R$ \\
\hline 1 & 0.395 & 0.360 & 0.400 & - & 0.100 & 0.100 & 0.100 & - \\
2 & $0.352^{*}$ & 0.248 & 0.333 & 0.054 & 0.100 & 0.100 & 0.100 & - \\
3 & 0.143 & 0.096 & 0.167 & - & 0.10 & 0.100 & 0.100 & - \\
4 & 0.026 & 0.00 & 0.044 & - & 0.100 & 0.100 & 0.100 & - \\
5 & 0.025 & 0.00 & 0.044 & - & 0.100 & 0.100 & 0.100 & - \\
6 & 0.026 & 0.00 & 0.044 & - & 0.100 & 0.100 & 0.100 & - \\
7 & 0.143 & 0.096 & 0.167 & - & 0.100 & 0.100 & 0.100 & - \\
8 & $0.352^{*}$ & 0.248 & 0.333 & 0.054 & 0.100 & 0.100 & 0.100 & - \\
9 & 0.395 & 0.360 & 0.400 & - & 0.100 & 0.100 & 0.100 & - \\
\hline
\end{tabular}

well in the effective and ineffective carrier sensing scenarios using tworay ground propagation model with saturated and unsaturated traffic demands.

\subsection{Simulation Validation with Two-ray Shadow- ing Propagation Model}

This section uses the two-ray shadowing propagation model [66] in the simulations to demonstrate the generalizability of the new model. The simulation results are presented in two parts: saturated and unsaturated traffic demands.

Two application scenarios are selected from the applications in the indoor built environments. The topology in Figure 3.6 is based on the scenario of the WiFi network at a building in the Kelburn campus of Victoria University of Wellington, Wellington, New Zealand. All the routers are placed within each other's carrier sensing range. Another applica- 
tion scenario in Figure 3.7 is based on the smart grid in a residential area in Wellington, New Zealand. The whole area exceeds the carrier sensing range.

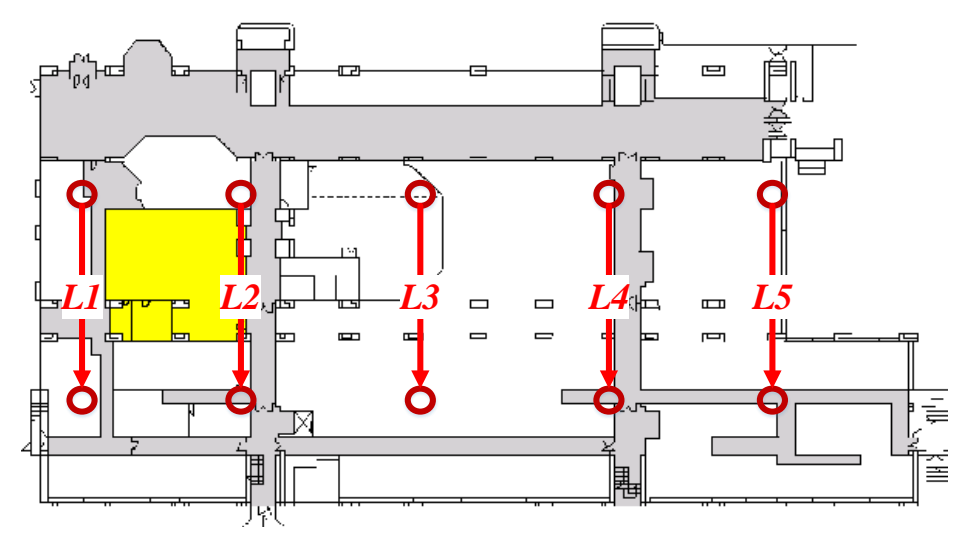

Figure 3.6: An example of the IEEE 802.11 WBN in a building representing two-ray shadowing ground propagation model

For two-ray ground shadowing model, three shadowing attenuations $\delta$ are selected as 2,4 , and 6 . The greater number of shadowing attenuation, the more obstacles exist in the propagation path. The higher the value of shadowing attenuation $\delta$ is, the greater the number of obstructions along the propagation path is assumed to be. The carrier sensing range $R_{C S}$ for $\delta$ as 2,4 ,and 6 is $430 \mathrm{~m}, 340 \mathrm{~m}$, and $270 \mathrm{~m}$ respectively.

\subsubsection{Saturated Traffic Demand}

For saturated traffic demand, two topologies are selected representing effective and ineffective carrier sensing scenarios. Table 3.12 shows the results of an effective carrier sensing scenario $(D=200 \mathrm{~m})$. The results show that adding a shadowing factor in the propagation model does not change the goodput pattern observed in two-ray propagation model. We found that the links in the effective carrier sensing scenario do not share the channel capacity equally. There is a small variation in goodput among 


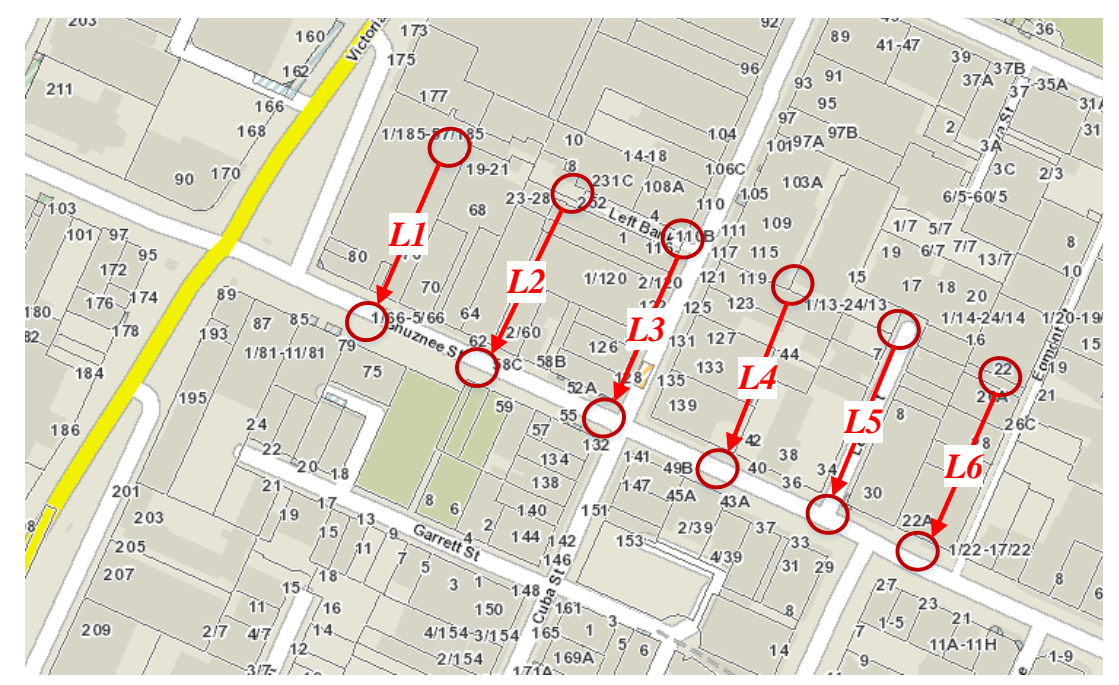

Figure 3.7: An example of the IEEE 802.11 WBN in central business district of Wellington representing two-ray shadowing ground propagation model

all the links. Along the same lines of explanation in Section 3.2.1, in effective carrier sensing scenarios, the randomised binary exponential back-off scheme used in IEEE 802.11 carrier sensing may result in a slight difference among the goodput of neighbouring links rather than achieving extreme

Table 3.12: Goodput of five links in a $200 \times 50 \mathrm{~m}^{2}$ topology with two-ray shadowing propagation model using saturated traffic

\begin{tabular}{c|c|cc|cc|cc}
\hline \multirow{2}{*}{ Link $i$} & \multirow{2}{*}{$G(i)$} & \multicolumn{2}{|c|}{ Shadowing $(\delta=2)$} & \multicolumn{2}{|c|}{ Shadowing $(\delta=4)$} & \multicolumn{2}{|c}{ Shadowing $(\delta=6)$} \\
& $G_{S}(i)$ & $E R$ & $G_{S}(i)$ & $E R$ & $G_{S}(i)$ & $E R$ \\
\hline 1 & 0.200 & $0.229^{*}$ & 0.127 & $0.203^{*}$ & 0.015 & 0.185 & - \\
2 & 0.200 & 0.158 & - & 0.174 & - & 0.180 & - \\
3 & 0.200 & 0.156 & - & 0.176 & - & 0.199 & - \\
4 & 0.200 & 0.159 & - & 0.174 & - & 0.181 & - \\
5 & 0.200 & $0.229^{*}$ & 0.127 & $0.203^{*}$ & 0.015 & 0.185 & - \\
\hline
\end{tabular}


Table 3.13: Goodput of six links in a $500 \times 50 \mathrm{~m}^{2}$ topology with two-ray shadowing propagation model using saturated traffic

\begin{tabular}{c|cccc|cccc|cccc}
\hline \multirow{2}{*}{ Link $i$} & \multicolumn{5}{|c|}{ Shadowing $(\delta=2)$} & \multicolumn{5}{c|}{ Shadowing $(\delta=4)$} & \multicolumn{5}{c}{ Shadowing $(\delta=6)$} \\
\hline 1 & $G_{S}(i)$ & $G_{P}(i)$ & $G_{O}(i)$ & $E R$ & $G_{S}(i)$ & $G_{P}(i)$ & $G_{O}(i)$ & $E R$ & $G_{S}(i)$ & $G_{P}(i)$ & $G_{O}(i)$ & $E R$ \\
\hline 2 & 0.837 & 0.733 & 1.000 & - & 0.628 & 0.578 & 0.667 & - & $0.571^{*}$ & 0.450 & 0.500 & 0.124 \\
3 & 0.044 & 0.000 & 0.067 & - & 0.277 & 0.217 & 0.333 & - & $0.217^{*}$ & 0.286 & 0.333 & 0.318 \\
4 & 0.039 & 0.000 & 0.067 & - & 0.026 & 0.00 & 0.067 & - & $0.168^{*}$ & 0.111 & 0.167 & 0.006 \\
5 & 0.039 & 0.000 & 0.067 & - & 0.026 & 0.00 & 0.067 & - & $0.168^{*}$ & 0.111 & 0.167 & 0.006 \\
6 & 0.044 & 0.000 & 0.067 & - & 0.275 & 0.217 & 0.333 & - & $0.218^{*}$ & 0.286 & 0.333 & 0.312 \\
& 0.837 & 0.733 & 1.000 & - & 0.629 & 0.578 & 0.667 & - & $0.570^{*}$ & 0.450 & 0.500 & 0.124 \\
\hline
\end{tabular}

equal goodput for each link [10]. In Table 3.12, the overall error is below $13 \%$.

For ineffective scenarios, the topology is chosen with the fixed border distance, $500 \mathrm{~m}$ and $d$ as $100 \mathrm{~m}$. Table 3.13 shows that the proposed model predicts starvation and goodput distribution accurately except the case $\delta=6$ that has the average error ratio of $16 \%$. It implies that the high shadowing factor brings more interaction between border links and middle links.

Table 3.14: Goodput of five links in a $200 \times 50 \mathrm{~m}^{2}$ topology with two-ray shadowing propagation model using saturated $\operatorname{traffic}(\delta=4)$

\begin{tabular}{c|cccc|cccc}
\hline Link $i$ & $f(i)$ & $G_{S}(i)$ & $G(i)$ & $E R$ & $f(i)$ & $G_{S}(i)$ & $G(i)$ & $E R$ \\
\hline 1 & 0.8 & $0.203^{*}$ & 0.200 & 0.013 & 0.6 & $0.204^{*}$ & 0.200 & 0.020 \\
2 & 0.8 & 0.174 & 0.200 & - & 0.6 & 0.173 & 0.200 & - \\
3 & 0.8 & 0.174 & 0.200 & - & 0.6 & 0.175 & 0.200 & - \\
4 & 0.8 & 0.174 & 0.200 & - & 0.6 & 0.174 & 0.200 & - \\
5 & 0.8 & $0.204^{*}$ & 0.200 & 0.020 & 0.6 & $0.204^{*}$ & 0.200 & 0.020 \\
\hline
\end{tabular}


Table 3.15: Goodput of five links in a $200 \times 50 \mathrm{~m}^{2}$ topology with two-ray shadowing propagation model using saturated traffic $(\delta=4)$-continued

\begin{tabular}{c|cccc|cccc}
\hline Link $i$ & $f(i)$ & $G_{S}(i)$ & $G(i)$ & $E R$ & $f(i)$ & $G_{S}(i)$ & $G(i)$ & $E R$ \\
\hline 1 & 0.4 & $0.203^{*}$ & 0.200 & 0.015 & 0.1 & 0.100 & 0.100 & - \\
2 & 0.4 & 0.174 & 0.200 & - & 0.1 & 0.100 & 0.100 & - \\
3 & 0.4 & 0.175 & 0.200 & - & 0.1 & 0.100 & 0.100 & - \\
4 & 0.4 & 0.174 & 0.200 & - & 0.1 & 0.100 & 0.100 & - \\
5 & 0.4 & $0.203^{*}$ & 0.200 & 0.015 & 0.1 & 0.100 & 0.100 & - \\
\hline
\end{tabular}

\subsubsection{Unsaturated Traffic Demand}

For unsaturated traffic demand, only the results with shadowing attenuation $\delta$ as 4 is listed due to space. Shadowing attenuation $\delta=4$ is the default value in QualNet 5.2 that refers to the common indoor environments with four walls.

Table 3.16: Goodput of six links in a $500 \times 50 \mathrm{~m}^{2}$ topology with two-ray shadowing propagation model $(\delta=4)$

\begin{tabular}{c|cccc|cccc}
\hline & \multicolumn{5}{|c|}{$f(i)=0.8$} & \multicolumn{4}{c}{$f(i)=0.6$} \\
Link $i$ & $G_{S}(i)$ & $G_{O}(i)$ & $G_{P}(i)$ & $E R$ & $G_{S}(i)$ & $G_{O}(i)$ & $G_{P}(i)$ & $E R$ \\
\hline 1 & 0.629 & 0.578 & 0.667 & - & 0.592 & 0.540 & 0.600 & - \\
2 & 0.276 & 0.217 & 0.333 & - & 0.276 & 0.217 & 0.333 & - \\
3 & 0.026 & 0.000 & 0.067 & - & 0.035 & 0.000 & 0.067 & - \\
4 & 0.026 & 0.000 & 0.067 & - & 0.034 & 0.000 & 0.067 & - \\
5 & 0.275 & 0.250 & 0.333 & - & 0.276 & 0.217 & 0.333 & - \\
6 & 0.629 & 0.578 & 0.667 & - & 0.592 & 0.540 & 0.600 & - \\
\hline
\end{tabular}

Tables 3.14 to 3.15 list the results from the effective carrier sensing scenario $(D=200 \mathrm{~m})$. When the sum of traffic demands of all links exceed the channel capacity, the difference of goodput between middle links and border links exists. The overall error is below $2 \%$. 
Table 3.17: Goodput of six links in a $500 \times 50 \mathrm{~m}^{2}$ topology with two-ray shadowing propagation model $(\delta=4)$-continued

\begin{tabular}{c|cccc|cccc}
\hline & \multicolumn{5}{|c|}{$f(i)=0.4$} & \multicolumn{4}{c}{$f(i)=0.1$} \\
Link $i$ & $G_{S}(i)$ & $G_{P}(i)$ & $G_{O}(i)$ & $E R$ & $G_{S}(i)$ & $G_{P}(i)$ & $G_{O}(i)$ & $E R$ \\
\hline 1 & 0.395 & 0.360 & 0.400 & - & 0.1 & 0.100 & 0.100 & - \\
2 & 0.311 & 0.217 & 0.333 & - & 0.1 & 0.100 & 0.100 & - \\
3 & 0.099 & 0.090 & 0.100 & - & 0.1 & 0.100 & 0.100 & - \\
4 & 0.099 & 0.090 & 0.100 & - & 0.1 & 0.100 & 0.100 & - \\
5 & 0.311 & 0.217 & 0.333 & - & 0.1 & 0.100 & 0.100 & - \\
6 & 0.395 & 0.360 & 0.400 & - & 0.1 & 0.100 & 0.100 & - \\
\hline
\end{tabular}

For the ineffective carrier sensing scenario $(D=500 \mathrm{~m}$ and $d=100 \mathrm{~m})$, the proposed model can predict starving links and goodput distribution correctly in Tables 3.16 to 3.17 .

Overall, the proposed goodput model performs well in the effective and ineffective carrier sensing scenarios using two-ray shadowing ground propagation model with saturated and unsaturated traffic demands. The prediction of starvation is accurate. Some errors exists in the prediction of goodput in effective carrier sensing scenarios. It implies that even in effective carrier sensing scenarios, the carrier sensing scheme cannot control the media access among neighbouring links fairly.

\subsection{Summary}

This chapter aims to answer the first research question: "How can a goodput distribution model provide an accurate prediction of the behaviour of wireless links in an IEEE 802.11 WBN under various scenarios such as exposed nodes, hidden nodes and border effect?".

To improve IEEE 802.11 WBN planning, a new goodput distribution model is developed with consideration of topology, both effective and in- 
effective carrier sensing conditions, and saturated and unsaturated traffic demands. The proposed goodput model has been validated in different scenarios through simulation (see more validations using different data rate in Appendix $\mathrm{C}$ and more validations using random topologies in [118]). The results from simulations show that the new goodput model can predict correctly the dominating border links and the extent of starvations as well with different propagation models and network sizes.

Such a goodput model is useful for network planning in IEEE 802.1 WBNs from different aspects. First, the proposed model is simple and accurate to predict potential performance such as goodput and fairness in an IEEE 802.11 WBN. Moreover, this model can be easily integrated into a network design tool. Second, this model helps guide node placement to prevent flow starvation in the planning stage. Finally, this model is useful for optimising the IEEE 802.11 protocols such as channel assignment and routing with an accurate prediction of link quality.

The next sections will apply this goodput model to optimise channel assignment for improving fairness and goodput. 
74 CHAPTER 3. GOODPUT MODELLING FOR PLANNING WBNS 


\section{Chapter 4}

\section{Channel Assignment For Improving Fairness in IEEE 802.11 WBNs}

Numerous scenarios about flow starvation have been studied extensively in Chapter 3 and our previous work [117]. Such flow starvation influences network fairness and users' experience. With an increasing number of intelligent devices and the increased volume of transmitted data in IEEE 802.11 wireless backhaul networks (WBNs), users expect to gain a fair level of quality of service (QoS) such as bandwidth [112, 131]. Hence, fairness becomes an important issue to be addressed in IEEE 802.11 WBNs.

The purpose of this chapter is to resolve flow starvation and improve fairness via optimised channel assignment (CA) algorithms in IEEE 802.11 WBNs.

Fairness in IEEE 802.11 WBNs depends on how wireless resources are allocated among the nodes in the networks since the relevant resources, such as wireless spectrum, are scarce [71]. If the wireless resources are allocated unfairly among the nodes in IEEE 802.11 WBNs, resource starvation can happen and leads to severe QoS degradation [131]. For example, flow starvation caused by border effect in IEEE 802.11 WBNs leads to un- 
fair sharing of a channel among nodes [54, 90, 116]. Hence, it is necessary to resolve unfairness problems like flow starvation for improving fairness. Note that in this research, fairness in IEEE 802.11 WBNs is defined as the long term fairness measured from the system perspective.

Investigating flow starvation with $\mathrm{CA}$ algorithms is closely linked to the interference models. An interference model is embedded within CA algorithms to determine the level of interference between nodes or links. The estimation of interference is used for allocating channels to the nodes in IEEE 802.11 WBNs. An accurate and realistic interference model directly contributes towards the effectiveness of CA algorithms in estimating interference, eliminating border effect and flow starvation, and achieving desired QoS. Hence, it is necessary to examine interference models used by existing $\mathrm{CA}$ algorithms.

To better understand the root cause of flow starvation, an investigation has been conducted to study flow starvation with three different interference models used in CA algorithms (see Appendix D). This investigation aims to identify the shortcoming of existing interference models to guide CA algorithms to prevent flow starvation. The analysis in Appendix D shows that flow starvation leads to severe unfairness, which is caused by global interference between border links and middle links. CA algorithms cannot prevent flow starvation in ineffective carrier sensing scenarios because the interference models used in CA algorithms only consider local interference between a link and its neighbouring links. Hence, CA algorithms need a new interference model reflecting global interference to prevent flow starvation and improve fairness.

The objectives of this chapter are to:

1. Design a new traffic-aware interference model that extends existing interference models to reflect global interference (border effect) with consideration of traffic information.

2. Design a traffic-unaware anti-starvation channel assignment (TU- 
ASCA) algorithm using the new interference model under saturated traffic assumption and validate the TUASCA algorithm.

3. Design a traffic-aware anti-starvation channel assignment (TAASCA) algorithm based on the TUASCA algorithm under unsaturated traffic assumption and validate the TAASCA algorithm.

The rest of this chapter is organised as follows. Section 4.1 introduces a new traffic-aware interference model. Sections 4.2 and 4.3 describe the proposed TUASCA and TAASCA algorithms followed by the summary in Section 4.4 .

\subsection{A New Traffic-aware Interference Model}

This section introduces the design of a new traffic-aware interference model. Different from existing interference models that only reflect "local" interference among neighbouring links (see the discussion in Appendix D.5), the new traffic-aware interference model leverages the concept of global and local interference and forms the basis for optimised CA algorithm to prevent flow starvation and improve fairness. The reason for taking traffic information into account is that a wireless network may or may not experience global interference that depends on the traffic information (more details in Section 3.1.2 on page 53).

Next subsection introduces the assumptions for the new interference model and the definitions of local and global interference embedded in the new model with an example. In the end, a discussion demonstrates the effect of local and global interference on fairness in IEEE 802.11 WBNs.

\subsubsection{Assumptions}

For mathematical tractability of the new interference model, several assumptions are necessary to facilitate the discussion. The symbols for a few variables together appear with a brief explanation in Table. 4.1. 
Table 4.1: Notation: Symbols and their meanings.

\begin{tabular}{ll}
\hline Symbol & Explanation \\
\hline$E$ & The complete set of links in an IEEE 802.11 WBN \\
$N$ & The number of links in $E$ \\
$M$ & The set of available channels for $E$ \\
$P$ & The topology information of links in $E$ \\
$E_{\text {order }}$ & The set of links in $E$ ordered by a method \\
$R_{c s}$ & Carrier sensing range \\
$R_{I}$ & Interference range \\
$S I R_{t h r e s h o l d}$ & The threshold for Signal-To-Interference Ratio (SIR) \\
$D_{t r}$ & The Cartesian distance between a sender and a receiver in the same link \\
$d_{L, B}$ & The Cartesian distance between a link and the left border link based on the senders' location \\
$d_{R, B}$ & The Cartesian distance between a link and the right border link based on the senders' location \\
$d_{S, r}$ & The Cartesian distance between a sender and a receiver from two different links \\
$d_{S, S}$ & The Cartesian distance between two senders from two different links \\
$d$ & The inter-link distance interval \\
$D$ & The Cartesian distance between two border links based on the senders' location \\
$G I S^{\prime}{ }_{L B}$ & The initial global left border set \\
$G I S^{\prime}{ }_{R B}$ & The initial global right border set \\
$G I S_{L B}$ & The global left border set \\
$G I S_{R B}$ & The global right border set \\
$G I S_{M L}$ & The global middle-link set \\
$\gamma_{L}(i)$ & The local interference set of a given link $i$ \\
$\beta$ & Path loss exponent factor \\
$f(i)$ & The traffic demand of link $i$ \\
\hline
\end{tabular}

Assumption 9. A single-radio single-channel IEEE 802.11 WBN is assumed.

Assumption 10. All links share the channel capacity equally in the effective scenario with saturated traffic demand.

Assumption 11. In ineffective carrier sensing scenarios, border effect may exist that depends on the traffic demands.

Assumption 12. Capture effect and packet losses caused by collision are ignored.

Assumption 9 simplifies the multi-channel multi-radio (MCMR) problem as a single-channel single-radio (SCSR) system. Hence, a MCMR system can be regarded as the combination of multiple SCSR systems using 
different non-overlapped channels. Generally, no interference exists between non-overlapped channels and thus the new interference model does not need to consider the influence among different SCSR systems using different channels. Assumptions 10 and 11 are the conclusions that have been validated through well-known studies such as [27,28, 114]. Assumption 12 simplifies the problem by neglecting the interference caused by collision.

\subsubsection{Defining Local Interference}

In this research, the local interference generalises the geometric interference models discussed in Chapter 2. Such local interference refers to the interference between a link and its neighbouring links. Note that this research mainly focuses on the interference caused by capacity contention and neglect the interference caused by collision in Assumption 12. A link and its neighbouring links constitute an interference set and contend for channel capacity. Hence, local interference is defined by an interference set as in Def. 17 and Eq. (4.1).

Definition 17. Local interference set $\gamma_{L}$

For a given link $i$ in E of an IEEE 802.11 WBN, the local interference set,

$$
\gamma_{L}(i)=\left\{l \in E \backslash\{i\} \mid d_{s, s} \leq R_{\mathcal{C S}} \text { or } d_{s, s}>R_{c S}, d_{s, r} \leq R_{c S}\right\}
$$

where $d_{s, s}$ is the Cartesian distance between the sender nodes of link $l$ and link $i$, $d_{s, r}$ is the Cartesian distance between the sender of link $l$ and the receiver of link $i$, and $R_{c s}$ is the carrier sensing range.

\subsubsection{Defining Global Interference}

Different from the local interference, global interference is observed from the perspective of the whole network and reflects the potential interference between border links and middle links in an IEEE 802.11 WBN in ineffective carrier sensing scenarios. 
In the proposed interference model, global interference is a function of both geometric distance and traffic demand. There are two necessary and sufficient conditions for global interference to occur. First, when the distance between border links is greater than carrier sensing range, a subset of nodes are not within each other's carrier sensing range and hidden nodes exist.

$$
D>R_{C S}
$$

where $D$ is the border distance and $R_{c s}$ is the carrier sensing range in the given IEEE 802.11 WBN.

Second, if the sum of normalised traffic demands of the links in borderlink sets (i.e. $G I S_{L B}$ and $G I S_{R B}$ ) exceeds the normalised channel capacity 1. Note that the normalised traffic demand is calculated with respect to the maximum net capacity. Note that the maximum net capacity in this thesis refers to goodput defined in Section 2.1. This implies that border links will occupy the whole channel capacity and middle links may not get any opportunity to access the media.

The second condition is expressed as:

$$
\sum_{j \in\left\{G I S_{L B} \cap G I S_{R B}\right\}} f(j) \geq 1,
$$

where $G I S_{L B}$ and $G I S_{R B}$ are the global left and right border sets in the given IEEE 802.11 WBN. Note that normalised channel capacity and normalised traffic demand with respect to the maximum net capacity are used in Eq.(4.3) here. The global left and right border sets are defined in Def. 19 . More details about $G I S_{L B}$ and $G I S_{R B}$ can be found in [117].

Initial global border sets $\left(G I S^{\prime}{ }_{L B}\right.$ and $\left.G I S^{\prime}{ }_{R B}\right)$, global left and right border sets $\left(G I S_{L B}\right.$ and $\left.G I S_{R B}\right)$ and global middle-link set $G I S_{M L}$ are defined as follows:

Definition 18. Initial Global Border sets $G I S^{\prime}{ }_{L B}$ and $G I S^{\prime}{ }_{R B}$ :

Let $E$ denote the complete set of links in an IEEE 802.11 WBN. For E, the left 
initial border set $G I S^{\prime}{ }_{L B}$ and right initial border set $G I S^{\prime}{ }_{R B}$,

$$
\begin{aligned}
& G^{\prime} S^{\prime}{ }_{L B}=\left\{l \in E \mid d_{l, R B}>R_{c S}\right\}, \\
& \text { GIS' }_{R B}=\left\{l \in E \mid d_{l, L B}>R_{C S}\right\} \text {, }
\end{aligned}
$$

whereby $d_{l, L B}$ is the distance between a link $l$ and the left border link, $d_{l, R B}$ is the distance between a link $l$ and the right border link, and $R_{c S}$ is the carrier sensing range.

Global left and right border sets $\left(G I S_{L B}\right.$ and $\left.G I S_{R B}\right)$ are defined based on the initial global border sets $\left(G I S^{\prime}{ }_{L B}\right.$ and $\left.G S^{\prime}{ }_{R B}\right)$ as follows.

Definition 19. Global Border sets $G I S_{L B}$ and $G I S_{R B}$

Let $E$ denote the complete set of links in an IEEE 802.11 WBN. For E, the left global border set $G I S_{L B}$ and the right global border set GIS $S_{R B}$,

$$
\begin{aligned}
& G_{I S_{L B}}=\left\{l \in E \backslash\left\{i \in G I S^{\prime}{ }_{R B}\right\} \mid d_{l, i}>R_{C S}\right\}, \\
& G I S_{R B}=\left\{l \in E \backslash\left\{i \in G I S^{\prime}{ }_{L B}\right\} \mid d_{l, i}>R_{C S}\right\} .
\end{aligned}
$$

whereby $d_{l, i}$ is the Cartesian distance between sender nodes in link $l$ and link $i$ that belong to the left border set and right border set, $R_{c s}$ is the carrier sensing range.

Definition 20. Global Middle-link set $G I S_{M L}$ : Let E denote the complete set of links in an IEEE 802.11 WBN. For $E$, the middle link set $G I S_{M L}$,

$$
G I S_{M L}=\left\{l \in E \backslash\left\{i \in G I S_{L B} \text { or } \in G I S_{R B}\right\}\right\} .
$$

Note that the above global border sets and middle-link set are inherited from the border-link sets, the dominant border-link sets, and the middlelink set (see definitions in Section 3.1.1.2 on page 46). To help understand global interference sets, an example illustrates the selection of the three global interference sets in the topology shown in Figure 4.1. In this scenario, border distance $D$ is $1000 \mathrm{~m}$ and the link interval $d$ is $100 \mathrm{~m}$. The carrier sensing range $R_{C S}$ is $710 \mathrm{~m}$. Based on Def. 18, GIS $_{L B}=\{L 1, L 2, L 3\}$, 


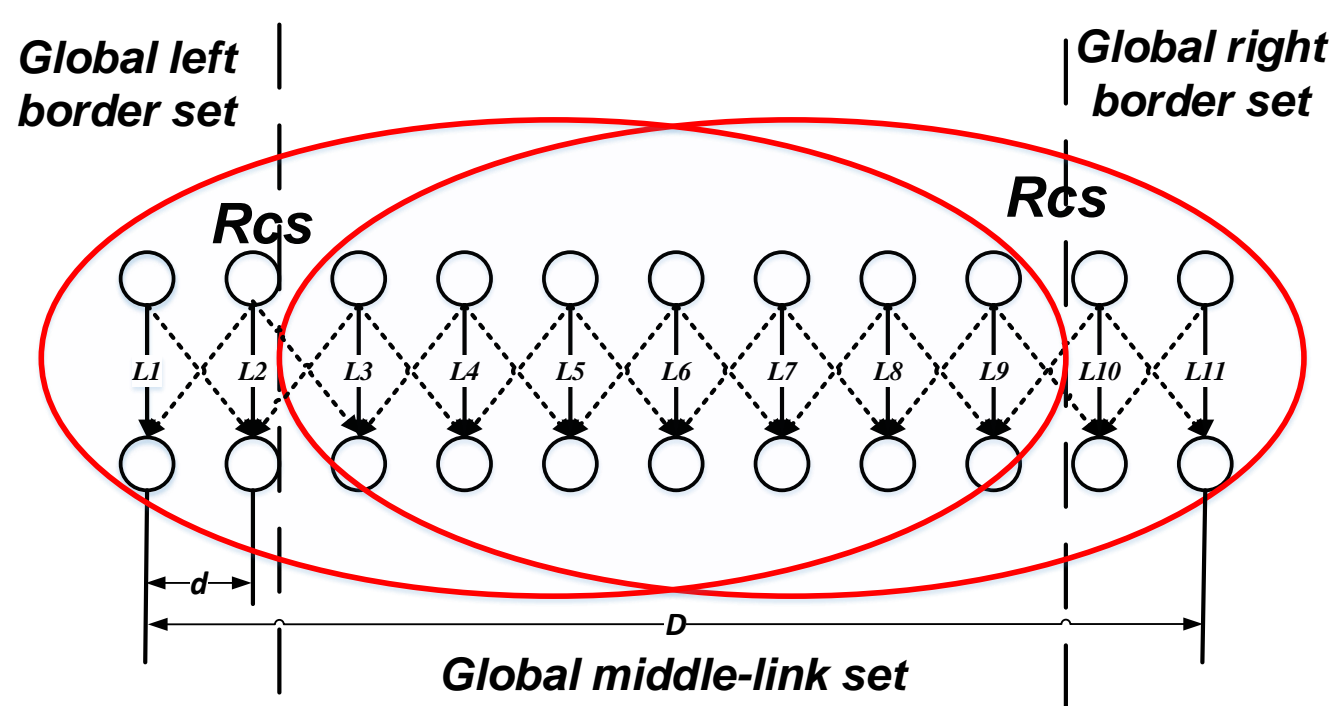

Figure 4.1: Selecting global interference sets

and GIS $_{R B}=\{L 9, L 10, L 11\}$. Based on Def. 19 and Def. 20, GIS $S_{L B}=$ $\{L 1, L 2\}, G I S_{R B}=\{L 10, L 11\}$, and $G I S_{M L}=\{L 3-L 9\}$. In Figure 4.1. two red circles denote the carrier sensing range of $G I S_{L B}$ and $G I S_{R B}$. The set of middle links $G I S_{M L}$ is defined to be within the carrier sensing ranges of both $G I S_{L B}$ and GIS $R B$. GIS $S_{L B}$ and GIS $R B$ are out of each other's the carrier sensing range. The global interference sets $G_{L B}, S_{L I} S_{R B}$, and GIS $S_{M L}$ will be used in the proposed CA algorithm to improve fairness. This scenario satisfies the first condition $D>R_{C S}$ in Eq. 4.2. If the sum of the traffic demands among the links in the global border sets satisfies Eq.( (4.3)), both local and global interference exist. If Eq.( (4.3) does not hold, only local interference exists and global interference does not exist. 


\subsubsection{Local and Global Interference with Fairness and Chan- nel Assignment}

Different from existing interference models, the proposed interference model reflects both local and global interference (border effect) with consideration of traffic demand. This subsection discusses the effect of local interference and global interference on fairness and how CA algorithms need to deal with local and global interference.

In effective carrier sensing scenarios, all nodes can sense each other so only local interference exists. Without global interference, border effect does not exist and flow starvation would not happen. The local interference between a link and its interfering links normally causes the variation of goodput among these links rather than severe fairness degradation. Hence, CA algorithms only need to mitigate local interference by utilising the diversity of channels.

For ineffective carrier sensing scenarios where not all nodes are within each other's carrier sensing range, if the sum of the traffic demands among the links in the global border sets satisfies $\sum_{j \in\left\{G I S_{L B} \cap G I S_{R B}\right\}} f(j) \geq 1$ in Eq. (4.3), both local and global interference exist. The global interference may cause flow starvation that can lead to significant fairness degradation. Therefore, global interference is the main cause of unfairness in IEEE 802.11 WBNs compared with local interference. To achieve better fairness in IEEE 802.11 WBNs, CA algorithms need to first resolve flow starvation caused by global interference and then alleviate local interference. If $\sum_{j \in\left\{G I S_{L B} \cap G I S_{R B}\right\}} f(j) \geq 1$ in Eq. (4.3) does not hold, CA algorithms only need to mitigate local interference as global interference does not exist.

The proposed interference model is based on the goodput model defined in Chapter 3. The validation of the goodput model shows that this goodput model can accurately identify flow starvation. This validation can be used to prove that this interference model can accurately model global interference (flow starvation). 
Next sections will apply the new traffic-aware interference model to design new CA algorithms to prevent flow starvation and improve fairness in IEEE 802.11 WBNs.

\subsection{A Traffic-unaware Anti-starvation Channel As- signment Algorithm For Improving Fairness}

In this section, a new traffic-unaware Anti-Starvation Channel Assignment (TUASCA) algorithm is designed to resolve flow starvation and improve fairness among the links under saturated traffic assumption in an IEEE 802.11 WBN. The reason to select saturated traffic demand is to simplify the problem that all links are equally configured with such saturated traffic. Saturated traffic demand can also be regarded as the extremely heavy traffic scenario. With saturated traffic demands, border effect is very likely to exist in ineffective carrier sensing scenarios (more details in Section 4.1.3).

Next subsections first introduce the design of this TUASCA algorithm and then validate TUASCA algorithm through simulation. The pros and cons of TUASCA algorithm will be discussed in the end.

\subsubsection{The Design of Traffic-unaware Anti-starvation Chan- nel Assignment Algorithm}

The TUASCA algorithm is designed as a static CA algorithm to prevent flow starvation and improve fairness for optimising IEEE 802.11 WBN planning. The inputs of the TUASCA algorithm are: (i) available channel set $C$, (ii) network link set $E$, and (iii) node position $P$ with fixed $R_{c s}$. The output is the channel allocation of all links in $E$.

Figure 4.2 shows the logic flow of the TUASCA algorithm. Firstly, the TUASCA algorithm checks whether the border distance $D$ of a given IEEE 802.11 WBN is greater than carrier sensing range $R_{C S}$. If this $\mathrm{WBN}$ is an 
effective carrier sensing scenario $\left(D \leq R_{C S}\right)$, the TUASCA algorithm alleviates local interference without spatial reuse by using a partition CA algorithm. The purpose of using a partition CA algorithm is to alleviate interference and achieve the fairness among subgroups using different channels.

If the given IEEE 802.11 WBN is an ineffective carrier sensing scenario $\left(D>R_{C S}\right)$, the TUASCA algorithm needs to check whether global interference exists or not. Under saturated traffic assumption, global interference exists in ineffective carrier sensing scenarios (see Section 3.1.1 on page 45). Next, the TUASCA checks whether the capacity condition is satisfied or not. The capacity condition is expressed as:

$$
|\gamma(B)|+1 \leq m,
$$

where $\gamma(B)$ is the local interference set of a border link $B$ and $m$ refers to the normalised capacity of all available channels. If $|\gamma(B)|+1 \leq m$ in Eq.( 4.7$)$ holds, it means the available channels are sufficient to directly address the potential global interference. Then the TUASCA algorithm solves global and local interference with spatial reuse.

If $|\gamma(B)|+1 \leq m$ in Eq. (4.7) does not hold, the available channel capacity is not sufficient to directly address the potential global interference. Next, the TUASCA algorithm starts to solve for both global and local interference with partial spatial reuse. According to the discussion in Section 4.1. CA algorithm needs to first resolve flow starvation caused by global interference and then alleviate local interference.

To solve the global interference in ineffective carrier sensing scenarios, the TUASCA algorithm first selects the global interference sets and formulates the objective function to achieve the fairness among global border sets and middle-link set as an Integer Linear Programming (ILP) problem in Def. 21. The objective is defined to achieve the maximum fairness by minimising the difference of average goodput between the global border sets and middle-link set. 


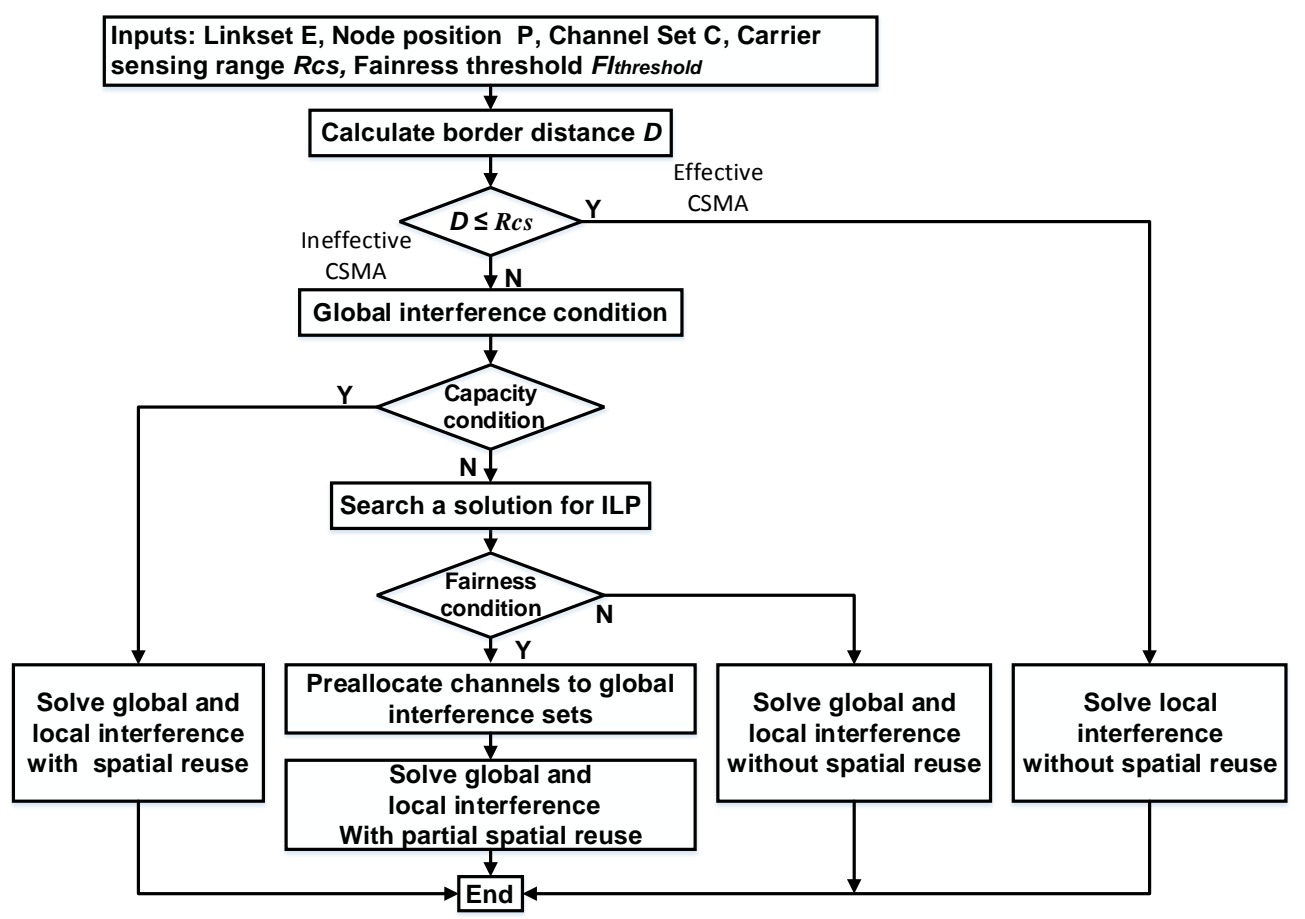

Figure 4.2: The logic flow of the TUASCA algorithm

In Def.21, $y$ is the variable that refers to the number of channels for the border set. $M,\left|G I S_{B}\right|$, and $N$ are three constant values from CA inputs denoting the number of available channels, the number of links in the global border set and the number of links in the whole network respectively.

Definition 21. Objective function: Let E denote the complete set of links in an IEEE 802.11 WBN. The objective is to:

maximise Fairness in $E$ via minimising the expression in 4.9 ,

$$
\begin{array}{rr}
\min \left(\frac{\mathrm{y}}{\left|G I S_{B}\right|}-\frac{M-\mathrm{y}}{N-2 \times\left|G I S_{B}\right|}\right), \\
\text { s.t. } \\
y \in Z^{+}, \\
0<y \leq M-1, \\
0<y \leq\left|G I S_{B}\right| .
\end{array}
$$


whereby $M$ is the available channel number, $y$ is the channel number for border sets, GIS $_{B}$ is the link number of one global border set, and $N$ is the link number in $E$.

To achieve fairness among three global interference sets, the TUASCA algorithm finds a $y$ representing the channel number allocated to the global border sets, which can minimise the difference of average goodput between global border sets and middle-link set in Eq.(4.9) by satisfying the constraint that $y$ is no more than the available channel number $M$ and the number of links in the global border set $\left|G S_{B}\right|$.

The formulation of the objective function in Def. 21 is essentially an Integer Linear Programming (ILP) problem seeking a $y$ that maximises fairness. Because the objective function has a single variable and is bounded by $M-1$, therefore the complexity of the ILP formulation is $O(M-1)$ where $M$ is the number of available channels [91].

Note that for a special case that the border set has very few links and the middle link set has a great amount of links, the solution from the ILP may lead to a low fairness. For example, $M=3,\left|G I S_{B}\right|=1, N=13$, in this case, the solution is $y=1$. The Jain's index could be around 0.5.

To address this extreme case, the TUASCA algorithm adds a fairness condition to check whether the Jain's index from ILP's solution will meet a satisfactory level of fairness $\left(F I_{\text {threshold }}\right)$. This fairness condition is expressed as:

$$
F I_{p}=\frac{(M+y)^{2}}{N \times\left(2 \times \frac{\mathrm{y}^{2}}{\left|G I S_{B}\right|}+\frac{(M-\mathrm{y})^{2}}{N-2 \times\left|G I S_{B}\right|}\right)} \geq F I_{\text {threshold }}
$$

whereby $F I_{p}$ is the estimate of potential Jain's index, $M$ is the available channel number, $y$ is the channel number for border sets from ILP, GIS $S_{B}$ is the link number of one global border set, $F I_{\text {threshold }}$ is to guarantee the TUASCA with a Jain's index above the pre-defined threshold, and $N$ is the link number in $E$.

If Eq. (4.10) does not hold, the potential fairness is not satisfactory so that the TUASCA algorithm will use the partition CA algorithm to pre- 
vent flow starvation and achieve better fairness. If Eq. (4.10) holds, the TUASCA algorithm use the solution from the ILP function to preallocates the channels to the global border sets and middle link set. The strategy to solve flow starvation in the TUASCA algorithm is to allocate distinct channels to global border sets and middle-link set. After that, the TUASCA algorithm selects a partition method to allocate preallocated channels to the links within each global interference set. The purpose of using a partition CA algorithm is to achieve the fairness among each links in each global interference set.

The design of the TUASCA algorithm is listed in Algorithm 1. First, the TUASCA algorithm orders with all links within $E$ based on Def. 19 with an increasing order (lines 3 of Algorithm 1). The local interference set of each link is selected based on Def. 17. In line 6 of Algorithm 1, the TUASCA algorithm calculates the network size $D$. If border distance $D \leq R_{C S}$ (an effective carrier sensing scenario), the TUASCA algorithm uses a partition method in Algorithm 2 to divide link set $E$ into subgroups based on the allocated channel number (see line 8 of Algorithm 1).

If border distance $D>R_{C S}$ (an ineffective carrier sensing scenario), the TUASCA algorithm first checks the capacity condition (see line 10 of Algorithm 1). If the capacity condition holds, the TUASCA algorithm reuses the spatial resource to address global interference and reduce local interference by selecting the least used channel in the local interference set to each link (see lines $11-13$ of Algorithm 1). If the capacity condition does not hold, the TUASCA algorithm selects the global interference sets (see lines 15 of Algorithm 1). The logic for constructing the global interference sets is listed in Algorithm 3 that first selects two global border sets in $E$ based on Def. 19 and then adds the remaining links to GIS $S_{M L}$. Subsequently, the TUASCA algorithm searches the optimal channel number $y$ to satisfy the ILP objective function in line 16 of Algorithm 1 . Then the TUASCA algorithm checks the fairness condition (see lines 17 of Algorithm 11. If the fairness condition holds, the TUASCA algorithm preallo- 


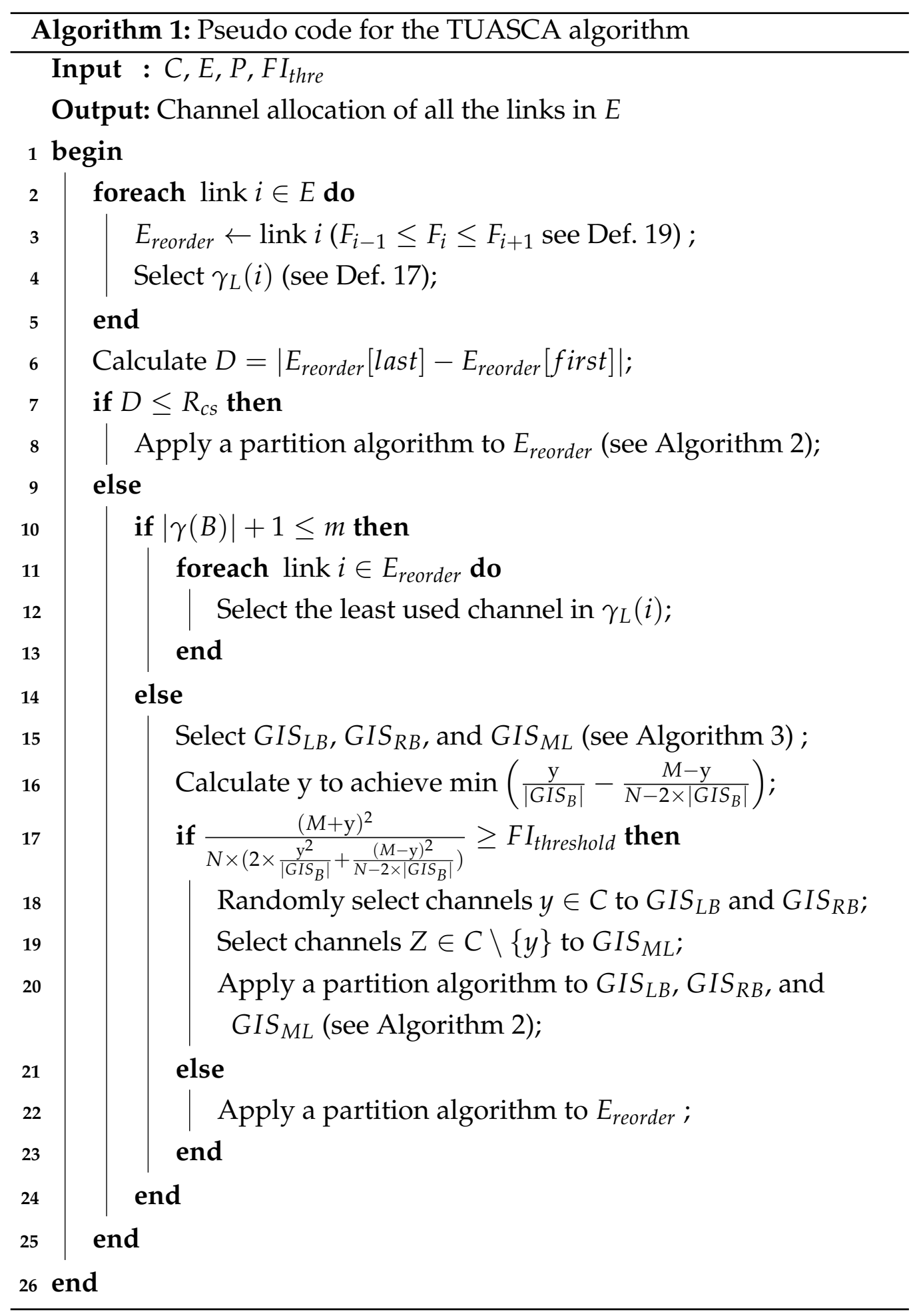




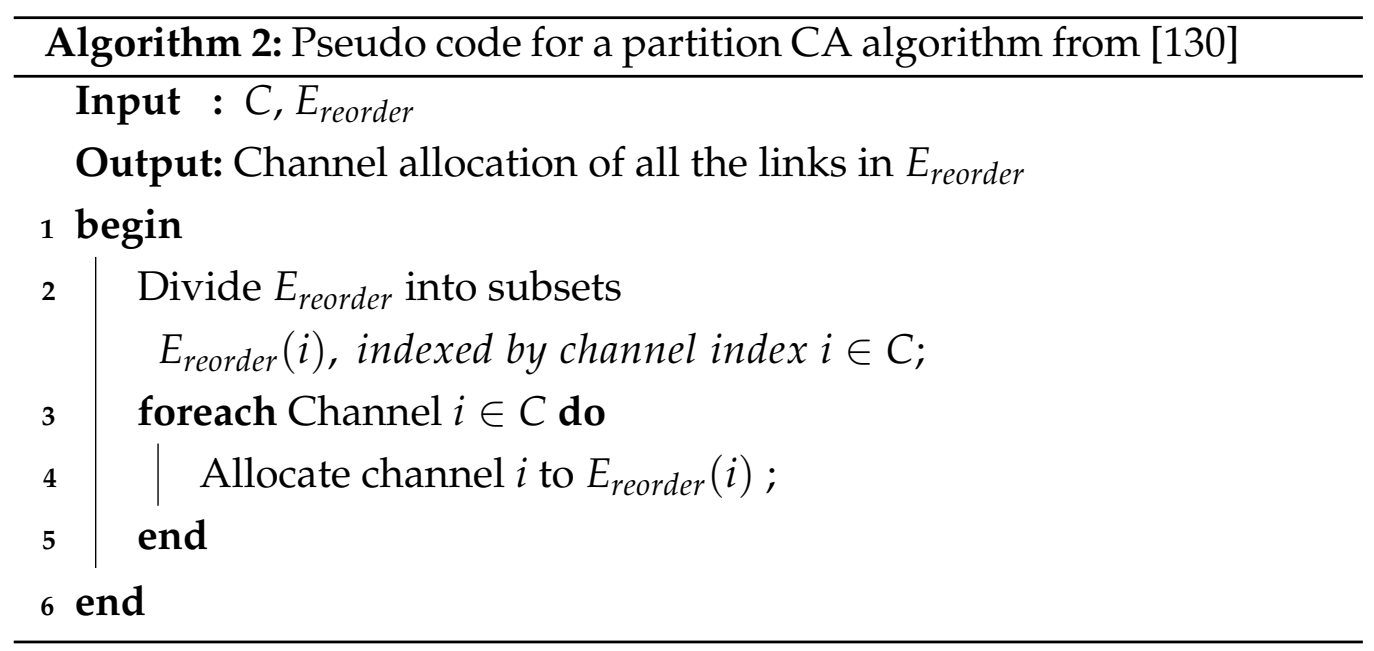

cates distinct channels to global border sets and middle-link set (see lines $18-19$ of Algorithm 1). Within each global interference sets, the TUASCA algorithm uses the partition method (see Algorithm 2 from [130]) to divide each global interference set into subgroups based on the allocated channel number (see line 20 of Algorithm 1). If the fairness condition does not hold, the TUASCA algorithm uses the partition method to allocate channels to the links in $E_{\text {reorder }}$ (see line 22 of Algorithm 1 ).

For ineffective carrier sensing scenarios with global interference and insufficient channel capacity, in the first phase, the TUASCA algorithm solves global interference by partitioning the global border sets and middlelink set with different channels and allocates identical channel to GIS $_{L B}$ and $G I S_{R B}$ to improve spatial reuse while in the second phase, the TUASCA algorithm minimises local interference within each global interference set.

The TUASCA algorithm utilises a simple partition method (c.f. Algorithm 2) to reduce the local interference. In Algorithm 2, the inputs are the link set $E_{\text {reorder }}$ and the available channel set $C$ and output is the channel allocation of the links in $E_{\text {reorder }}$. This partition CA algorithm divides the link set $E_{\text {reorder }}$ into groups where the number of groups is equal to the number of available channels (line 2 of Algorithm 2) and allocate each 


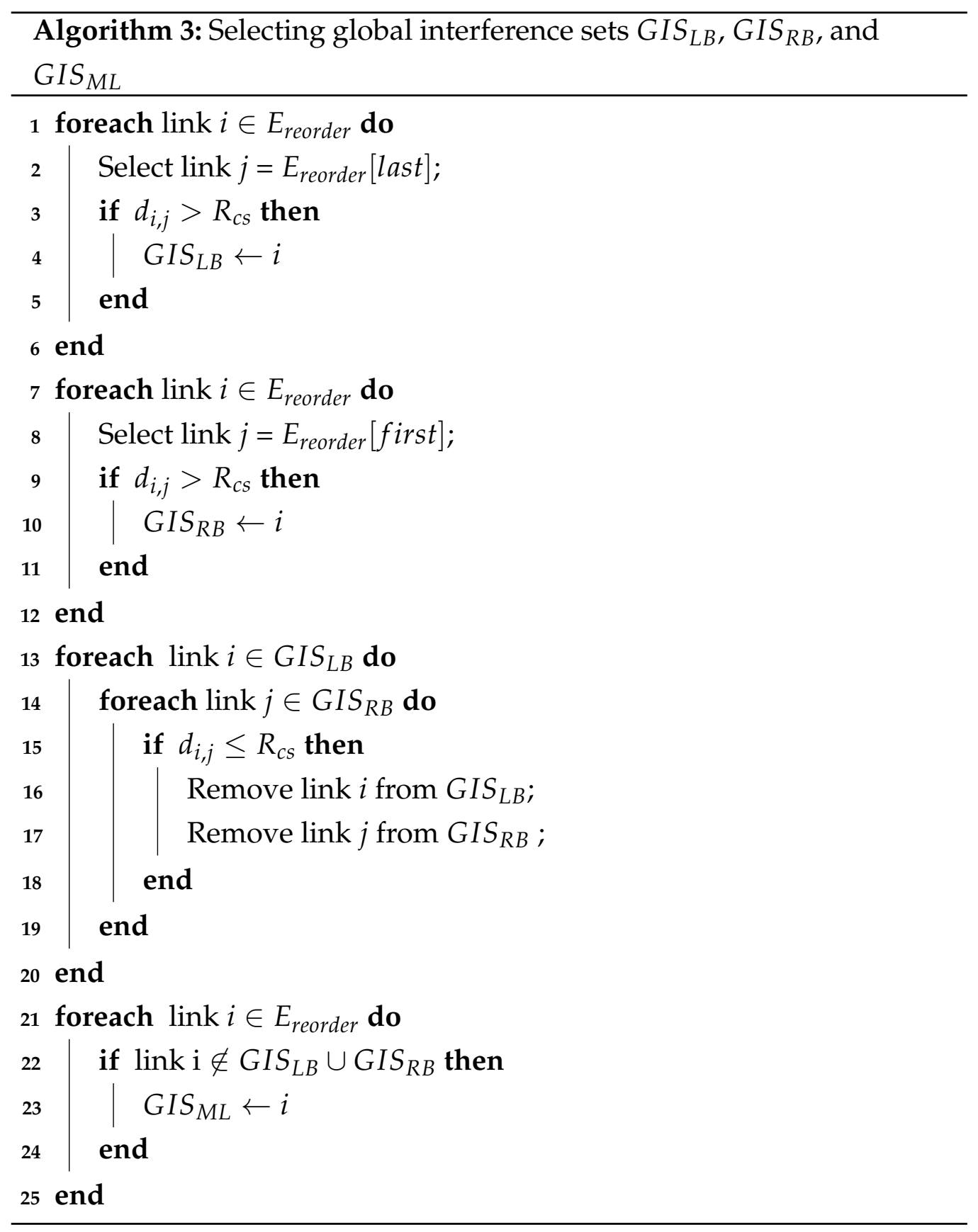


group with different channels (lines 3 - 5 of Algorithm 2). By using different channels in different groups, the partition CA algorithm mitigates the channel contention among different groups.

The next sections discuss the validation of TUASCA algorithm through simulation.

\subsubsection{Benchmark For Traffic-unaware Anti-starvation Chan- nel Assignment Algorithm}

A standard clique-based CA algorithm INSTC [140] discussed in Chapter 2 is selected as the benchmark for the TUASCA algorithm. The INSTC algorithm integrates three interference set selections based on three common interference models. The reason to select the clique-based CA algorithm is that clique-based CA algorithm is a common method among traffic-unaware CA algorithms discussed in Section 2.4 .2 on page 26. In this thesis, we only borrow the strategy of channel assignment in [140] for minimising interference without considering the maintenance of topology to simplify the problem.

Here, three interference set selections are defined based on three distinct interference models. Two categories of interference set selection strategies we select are: (i) carrier sensing oriented and (ii) packet reception oriented strategies. The carrier sensing oriented strategy focuses on the capacity contention between transmitter nodes while the packet reception oriented strategy pays particular attention to the interference at a receiver node from neighbouring nodes during the packet reception. With the carrier sensing oriented strategy, $\gamma_{A}$ is defined as carrier sensing oriented interference set. For the packet reception oriented strategy, two distinct interference sets, $\gamma_{B}$ and $\gamma_{C}$ are defined as follows. The definitions of these interference sets are listed below: 


\subsubsection{Carrier Sensing Oriented Strategy}

Definition 22. Interference set $\gamma_{A}$

Let $E$ denote the complete set of links in an IEEE 802.11 WBN. For a tagged link $i$ in $E$, the interference set,

$$
\gamma_{A}(i)=\left\{l \in E \backslash\{i\} \mid d_{s, s} \leq R_{c S} \text { or } d_{s, r} \leq R_{c S}\right\}
$$

whereby $d_{s, s}$ is the distance between the sender nodes of link $l$ and link $i, d_{s, r}$ is the distance between the sender of link $l$ and the receiver of link $i, R_{c S}$ is the carrier sensing range.

\subsubsection{Packet Reception Oriented Strategies}

\section{Definition 23. Interference set $\gamma_{B}$}

Let $E$ denote the complete set of links in an IEEE 802.11 WBN. For a tagged link $i$ in $E$, the interference set,

$$
\gamma_{B}(i)=\left\{l \in E \backslash\{i\} \mid d_{s, r} \leq R_{I}\right\},
$$

whereby $d_{s, r}$ is the distance between the sender of link $l$ and the receiver of link $i$, $R_{I}$ is the theoretical interference range $\left(R_{I}=k \times D_{t r}, k>0\right)$.

\section{Definition 24. Interference set $\gamma_{C}$}

Let $E$ denote the complete set of links in an IEEE 802.11 WBN. For a tagged link $i$ in $E$, the interference set,

$$
\gamma_{C}(i)=\left\{l \in E \backslash\{i\} \mid\left(\frac{d_{s, r}}{D_{t r}}\right)^{\beta} \leq S I R_{\text {thre }}\right\},
$$

whereby $d_{s, r}$ is the distance between the sender of link $l$ and the receiver of link $i, D_{t r}$ is the transmitter-receiver separation of link $i, \beta$ is the path loss exponent factor, $S I R_{\text {thre }}$ is the SIR threshold for a successful transmission subject to the used modulation and coding scheme. 


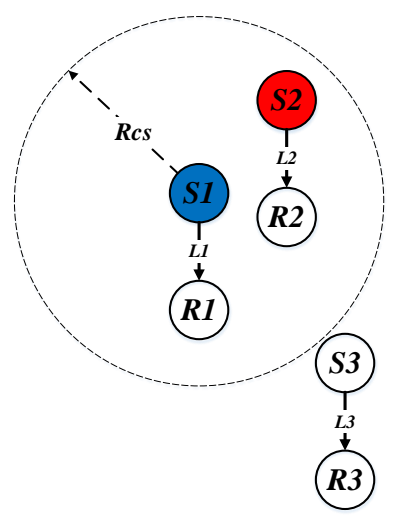

Figure 4.3: Visualising interference set $\gamma_{A}$

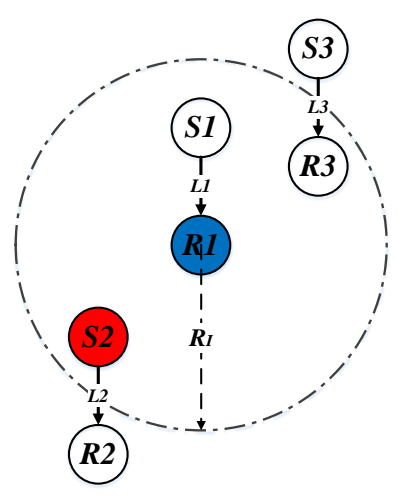

Figure 4.4: Visualising interference set $\gamma_{B}$

\subsubsection{Example: Selecting Interference Sets}

To help understand the difference between three different interference set selection strategies, Figures 4.3 and 4.4 demonstrate the selections of these interference sets. The node highlighted in red is in conflict with the node in blue assuming both nodes use the same channel.

Carrier sensing oriented interference set $\gamma_{A}$ mainly focuses on the capacity contention between sender nodes when they attempt to transmit packets concurrently. In Figure 4.3, according to Def. 4.11 of interference set $\gamma_{A}, \gamma_{A}(L 1)=\{L 2\}$ because sender $S 2$ of link $L 2$ is within the carrier sensing range of sender $S 1$ in link $L 1$.

The packet reception oriented interference sets $\gamma_{B}$ and $\gamma_{C}$ focus on the potential interference at the receiver node during the packet reception from its sender. The interference sets given by $\gamma_{B}$ is based on a theoretical interference model while the interference set $\gamma_{C}$ is based on the measurement-based interference model. In Figure 4.4, $\gamma_{B}(L 1)=\{L 2\}$ because sender $S 2$ of link $L 2$ is within the interference range $R_{i}$ of receiver $R 1$ of link $L 1$.

Figure 4.4 can also explain the selection of the interference set $\gamma_{C}$. Assume that a scenario matches with the two-ray ground propagation model [66] 
and IEEE 802.11b 2Mbps is used for applications in which $\beta=4$ and $S I R_{\text {thre }}=10$. According to Def. 4.13, $d_{s, r} \leq 1.78 \times D_{t r}$. In Figure 4.4 . $\gamma_{C}(L 1)=\{L 2\}$ if sender $S 2$ of link $L 2$ is within the interference range $R_{I}=1.78 \times D_{t r}(L 2)$ of receiver $R 1$ of link $L 1$.

\subsubsection{A Clique-based Channel Assignment algorithm}

The inputs of the clique-based INSTC CA algorithm are: (i) available channel set $C$, (ii) network link set $E$, and (iii) node position $P$ with fixed $R_{c s}$, $R_{I}$, and $S I R_{\text {thre }}$. The output is the channel allocation of all links in $E$.

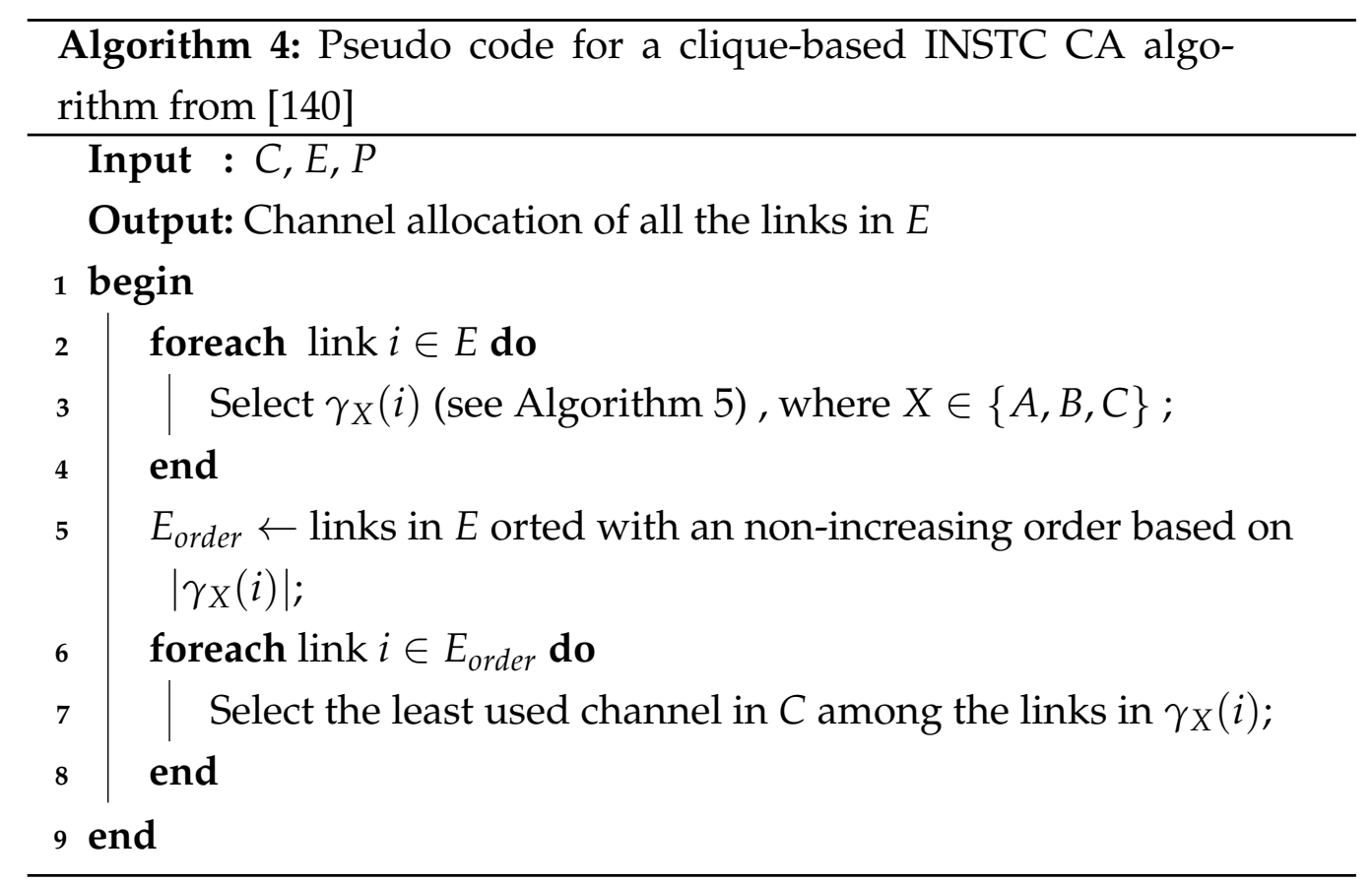

The INSTC CA algorithm has three main steps: (i) selecting the interference set for each link in $E$, (ii) ordering links, and (iii) allocating channels to links in $E_{\text {order }}$. In the first step (in lines $2-4$ of Algorithm 4), the CA algorithm selects the interference set of each link $i$ as $\gamma_{X}$ where $X \in\{A, B, C\}$ denotes the different strategies listed in Algorithm 5 In the second step, the link set $E_{\text {order }}$ is sorted in a non-increasing order of 


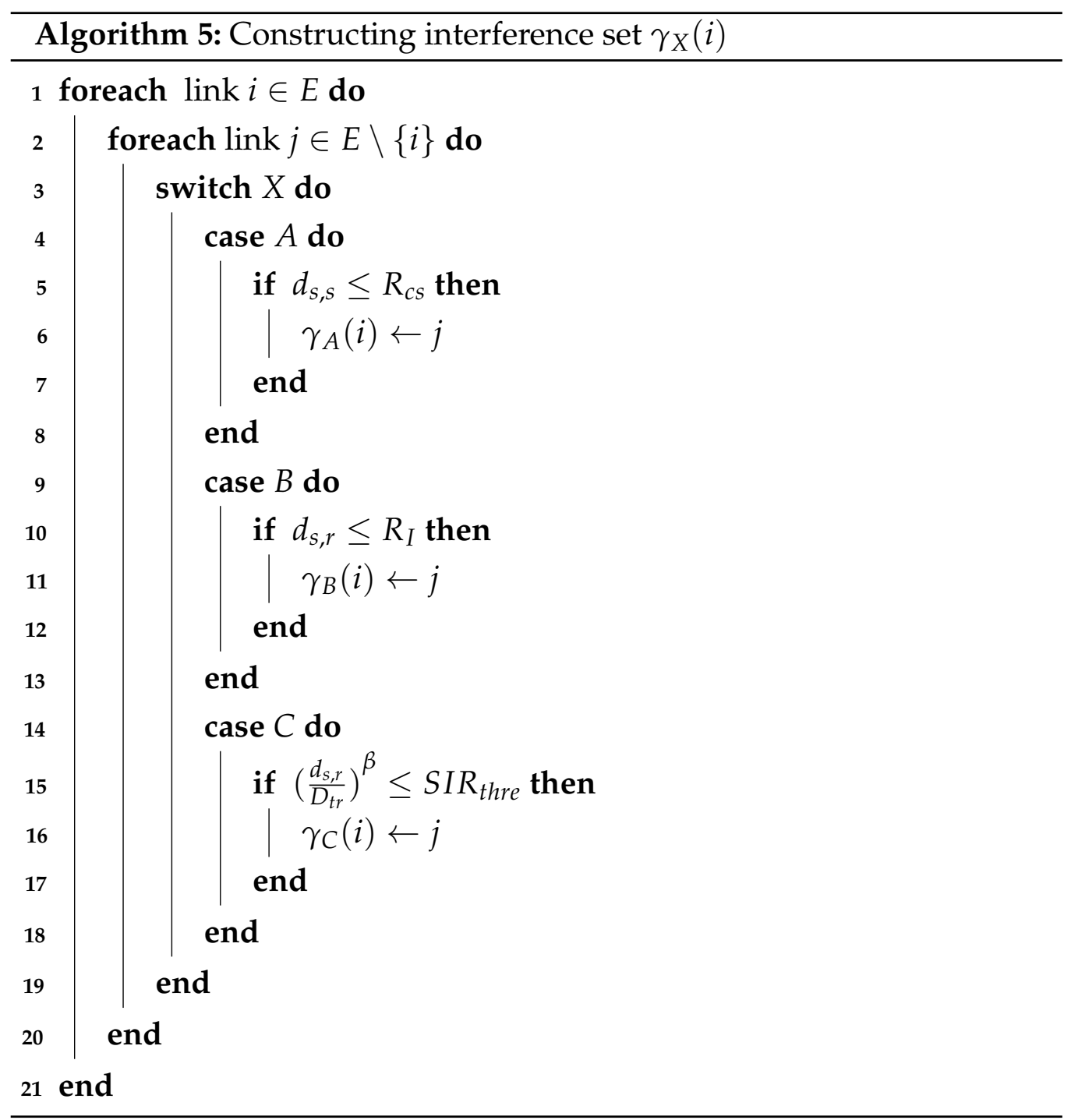

the Link Potential Interference (LPI) that is defined as the number of links within the interference set (line 5 of Algorithm 4). A simple bubble sorting algorithm is used to order the links in $E_{\text {order }}$ because our focus is the influence of the interference set selection on network fairness instead of the ordering method. Next, the CA algorithm allocates channels to the links in the ordered $E_{\text {order }}$ (lines 6-8 of Algorithm 4 ). The channel allocation strategy is such that the least frequently used channel within the interference 
set of a link $i$ is allocated to this link. Such a channel allocation strategy has been widely used in CA algorithms to reduce the interference among the links within an interference set.

Next subsections discuss the validation of the TUASCA algorithm with the above benchmark through simulation.

\subsubsection{Validation of Traffic-unaware Anti-starvation Chan- nel Assignment Algorithm with Three Channels}

This subsection shows the validation of the TUASCA algorithm with the benchmarks through simulation using three channels. The benchmarks are the clique-based algorithm using three different interference set selections $\gamma_{A}, \gamma_{B}$, and $\gamma_{C}$ (see Section 4.2.2).

\subsubsection{Simulation Configuration and Measurement Metrics}

The inputs of the TUASCA algorithm and the INSTC CA algorithm include available channel set, link set, and network topology. First, the available channels are set as $C=\{1,2,3\}$ to show how the TUASCA effectively prevent flow starvation and improve fairness with limited channels.

Second, grid topologies and random topologies are used ranging from small border distance $D=200 \mathrm{~m}$ to large border distance $D=800 \mathrm{~m}$. The link set ranges from 5 single-hop links to 17 single-hop links. The grid topologies used in this section deploy links with a constant interval $d$ of $50 \mathrm{~m}$ (see Figure 4.5). Random topologies we use are generated by a $\mathrm{R}$ script. With a given area and given link number, the node position and link length are generated randomly according to uniform distribution.

The simulation parameters are in Table 4.2. With the parameters in Table 4.2, $R_{c s}$ is calculated as $515 \mathrm{~m}, R_{I}$ is defined as $2 \times D_{t r}^{\max }\left(D_{t r}^{\max }\right.$ is $207 \mathrm{~m}), \beta$ as 4 , and $S I R_{\text {thre }}$ as $13 \mathrm{~dB}[1]$. Transmitter-receiver separation for all the links in simulation is selected as $50 \mathrm{~m}$ to guarantee collision-free transmissions (based on the findings from [156]) whereby it was found 
that carrier sensing mechanism can protect packet transmission against collision when the transmitter-receiver separation is less than $0.56 \times D_{t r}^{\max }$ ( $D_{t r}^{\max }$ denotes maximum transmission range). To simplify the analysis, all nodes are identically configured with saturated traffic generators. The threshold of Jain's index for the TUASCA algorithm is set as 0.8 that we assume it is a satisfactory level of fairness.

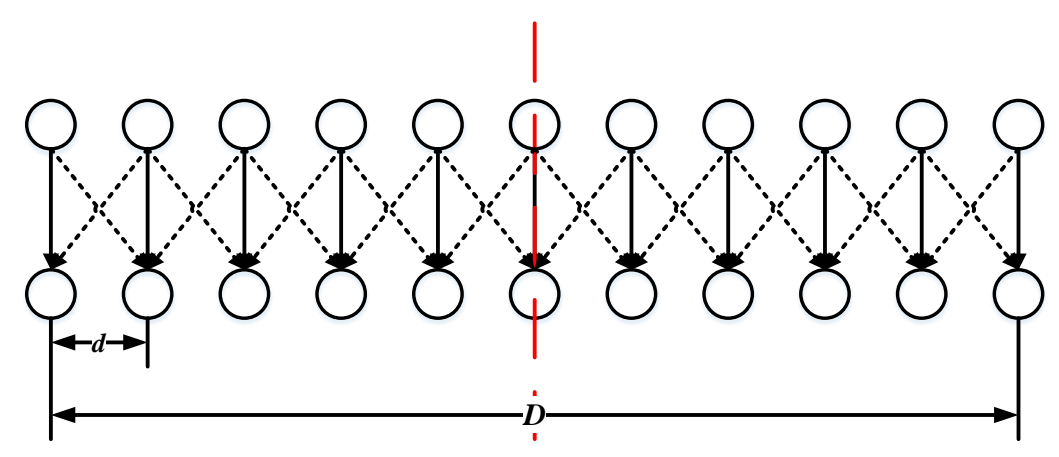

Figure 4.5: The grid topology used in the example

With the above inputs and the parameters in Table 4.2 , the TUASCA algorithm and its benchmark yield different channel allocations. Those channel allocations are implemented into the simulation tool, Qualnet 5.2. The average goodput of each links is calculated from 100 randomly seeded simulation runs. All averages of simulation results shown are reported with confidence interval of $95 \%$ with the range from $[5.2,15.66] \mathrm{kbps}$ under the assumption that the averages are normally distributed.

In order to evaluate the performance of the TUASCA algorithm, the validation considers four metrics:

- Jain's index [33]:

Definition 25. Jain's index FI 
Table 4.2: Simulation Parameters

\begin{tabular}{l|l}
\hline Parameter Name & Value \\
\hline Transmission Power & $18 \mathrm{dBm}$ \\
\hline Receiver Sensitivity & $-78 \mathrm{dBm}$ \\
\hline Path Loss Model & Two-Ray \\
\hline Shadowing \& Fading Model & None \\
\hline MAC Layer & PCS \\
\hline Routing & Static Routing \\
\hline Transportation Layer & UDP \\
\hline Physical Layer & IEEE802.11a \\
\hline Data Rate & $24 \mathrm{Mbps}$ \\
\hline Packet Size & $1500 \mathrm{Bytes}$ \\
\hline Interpacket Interval & $0.5 \mathrm{~ms}$ \\
\hline
\end{tabular}

Let $E$ denote the set of links in an IEEE 802.11 WBN,

$$
F I=\frac{\left(\sum_{i \in E} G_{S}(i)\right)^{2}}{N \times \sum_{i \in E}\left(G_{S}(i)\right)^{2}},
$$

where $G_{s}(i)$ is the normalised goodput of a link $i$ with respect to the maximum net capacity from simulation, $N$ is the number of links in $E$.

Jain's index FI provides an indication of the overall system fairness. The range of Jain's index is between 0 and 1 . The system is fairer when the Jain's index is closer to 1.

- Starvation link ratio $S R$ : The starvation link ratio $S R$ that is the ratio between the number of starvation links and the number of all links in an IEEE 802.11 WBN. Starvation link ratio reflects the percentage of starvation links among all links. In this research, a starvation link is defined as that the achieved goodput of a link is below $\alpha \times G_{\text {average, }}$ where $\alpha \in[0.0,0.2]$ is the starvation factor and $G_{\text {average }}$ is the average goodput in $E$. 
Definition 26. Starvation link ratio $S R$

Let $E$ denote the set of links in an IEEE 802.11 WBN,

$$
S R=\frac{\left|E_{\text {starvation }}\right|}{N}
$$

where $\left|E_{\text {starvation }}\right|$ is the number of links that are predicted to have flow starvation, $N$ is the number of links in $E$.

- Highest-to-lowest goodput ratio HLG [131]: Highest-to-lowest goodput ratio is the ratio between the highest achieved goodput and the lowest achieved goodput among all the links in an IEEE 802.11 WBN.

Definition 27. Highest-to-lowest goodput ratio HLG

Let $E$ denote the set of links in an IEEE 802.11 WBN,

$$
H L G=\frac{G_{\max }}{G_{\min }},
$$

where $G_{\max }$ and $G_{\min }$ are the maximum and minimum goodput values among all links in $E$ respectively.

- Normalised average goodput $A G$ : the normalised average goodput is a common metric to evaluate CA algorithms (see Section 2.4).

Definition 28. Normalised average goodput $A G$

For a given E of an IEEE 802.11 WBN,

$$
A G=\frac{\sum_{i \in E} G_{s}(i)}{N}
$$

where $G_{s}(i)$ is the normalised goodput of a link $i$ with respect to the maximum net capacity from simulation, $N$ is the number of links in $E$.

The next subsections will validate the TUASCA algorithm with the benchmark from the view of different fairness measures. 


\subsubsection{Comparison of Jain's Index}

Figures 4.6 and 4.7 show the comparison of Jain's index with corresponding 95\% confidence interval among the clique-based CA algorithm using interference set selections $\gamma_{A}, \gamma_{B}$, and $\gamma_{C}$, and TUASCA algorithm in grid and random topologies respectively. In Figures 4.6 and 4.7 , the $X$ axis denotes the border distance in Figure 4.5 from $200 \mathrm{~m}$ to $800 \mathrm{~m}$ while the $Y$ axis refers to the achieved Jain's index of the channel allocations from different algorithms. The 95\% confidence interval is also plotted in Figures 4.6 and 4.7 but the value of confidence interval is very small.

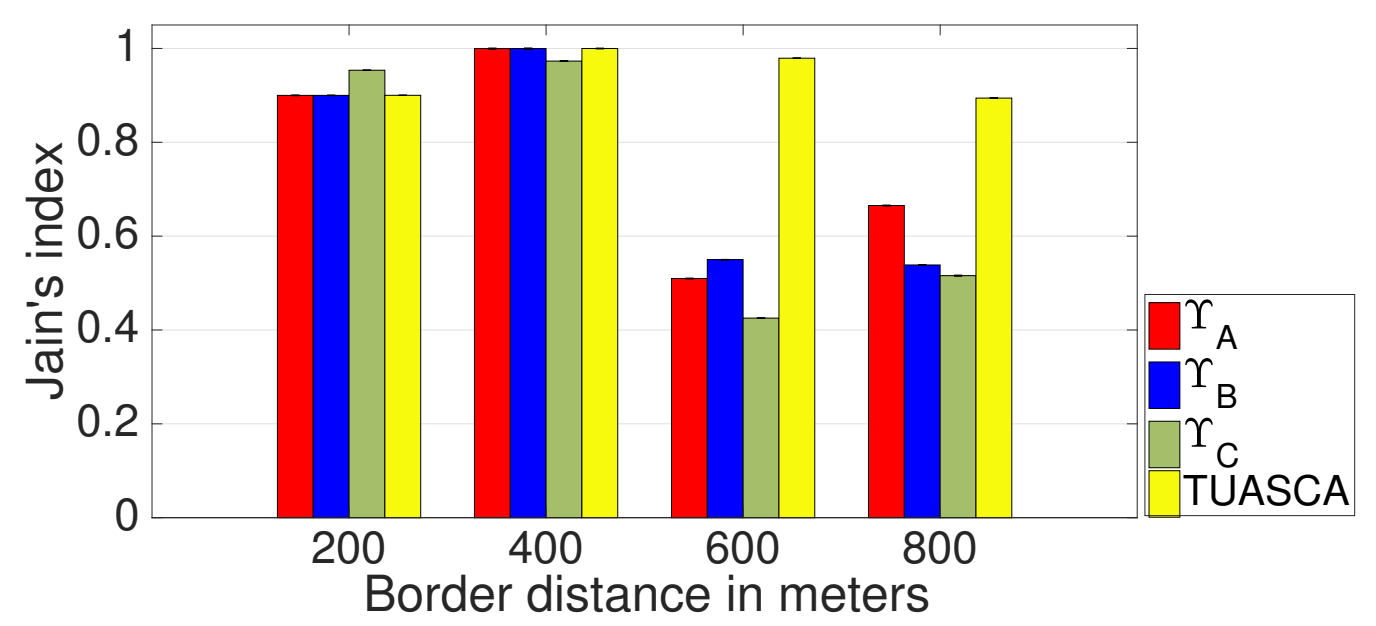

Figure 4.6: The comparison of Jain's index using three channels in grid topologies

In Figure 4.6, when the border distance is less than $R_{c s}$ (i.e. $200 \mathrm{~m}$ and $400 \mathrm{~m}$, the effective carrier sensing scenarios), all four CA algorithms achieve similar Jain's index. When the border distance is greater than $R_{c s}$ (i.e. between $600 \mathrm{~m}$ and $800 \mathrm{~m}$, the ineffective carrier sensing scenarios), the TUASCA algorithm achieves better Jain's index than other three CA algorithms.

Figure 4.7 shows a similar trend as that in Figure 4.6 . In effective car- 


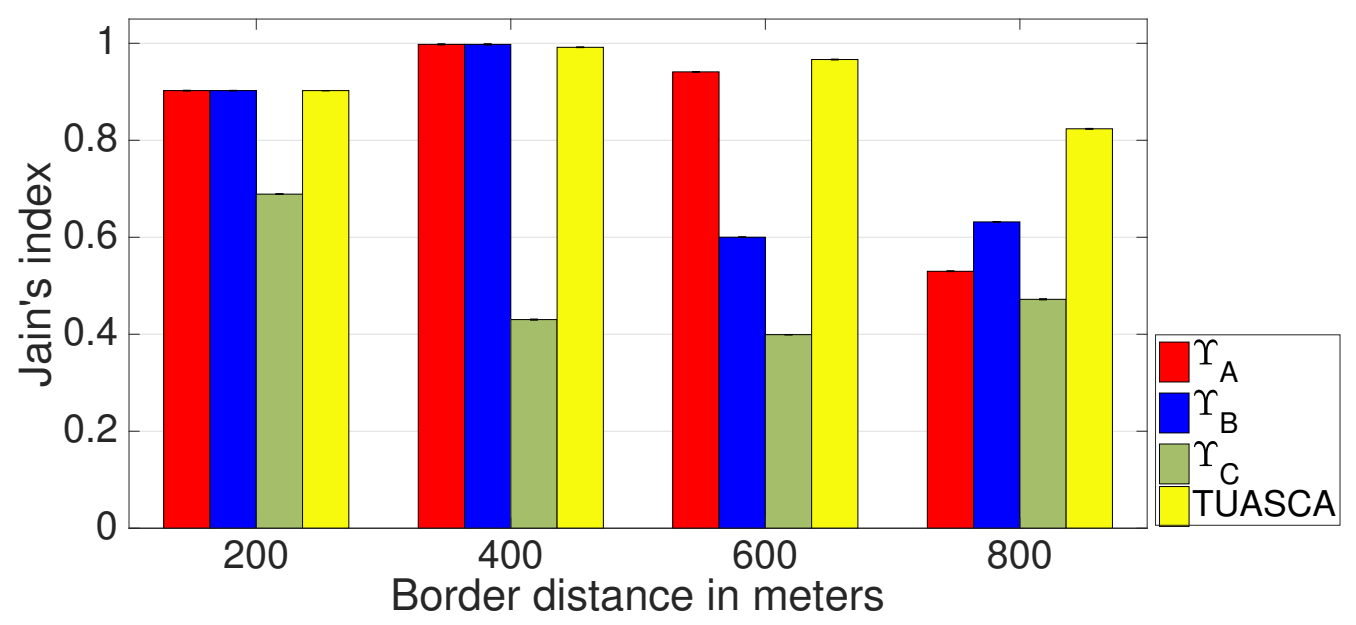

Figure 4.7: The comparison of Jain's index using three channels in random topologies

rier sensing scenarios, the clique-based CA using the interference sets $\gamma_{A}$ and $\gamma_{B}$ achieve the same Jain's index as the TUASCA, while the cliquebased CA using the interference set selection $\gamma_{C}$ achieves a lower Jain's index. In ineffective carrier sensing scenarios, TUASCA algorithm achieves the highest Jain's index among all CA algorithms. In the case $D$ as $600 \mathrm{~m}$ in Figure 4.7, we find one exception that the channel allocation from the clique-based CA using the interference set selection $\gamma_{A}$ achieves 0.94 .

Overall, in ineffective carrier sensing scenarios, the TUASCA algorithm achieves $34-92 \%$ better fairness compared with the selection strategy $\gamma_{A}$ (the overall highest among three interference sets) in terms of Jain's index.

\subsubsection{Comparison of Starvation Link Ratio}

Figures 4.8 and 4.9 show the comparison of starvation link ratio with corresponding 95\% confidence interval among the clique-based CA algorithm using interference set selections $\gamma_{A}, \gamma_{B}$, and $\gamma_{C}$, and TUASCA algorithm in grid and random topologies respectively. In Figures 4.8 and 4.9 , the $X$ 
axis denotes the border distance in Figure 4.5 while the $Y$ axis refers to the starvation link ratio of different CA algorithms.

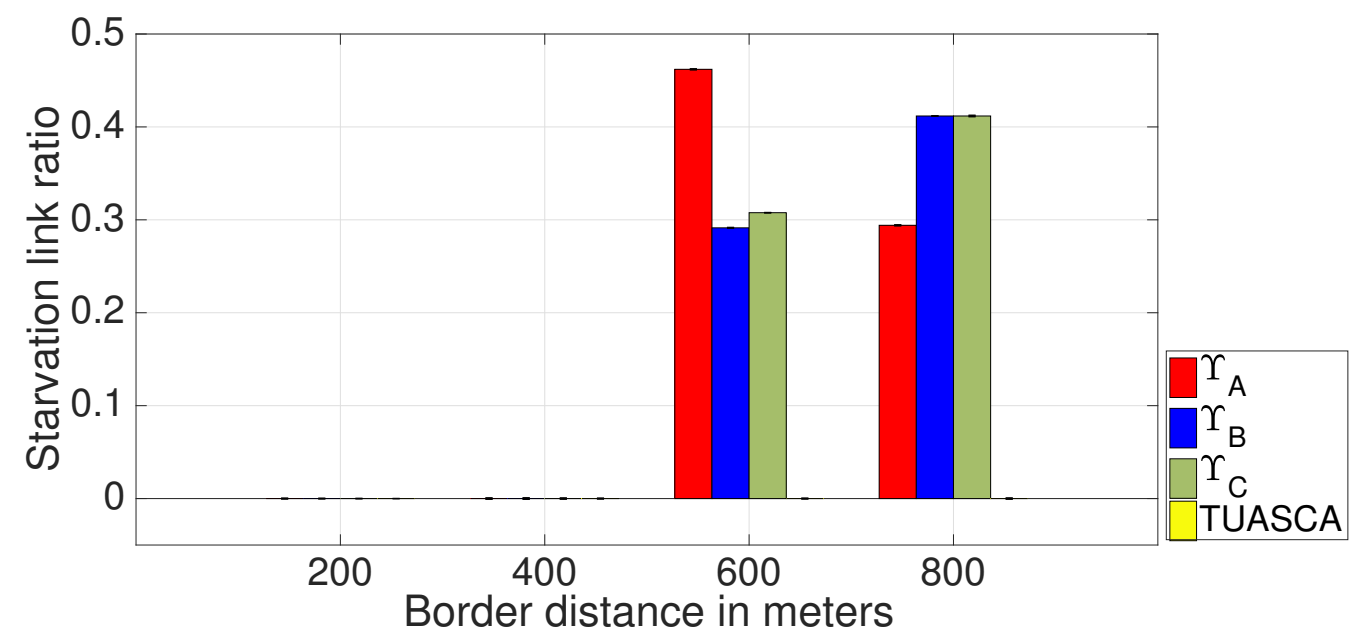

Figure 4.8: The comparison of starvation link ratio using three channels in grid topologies

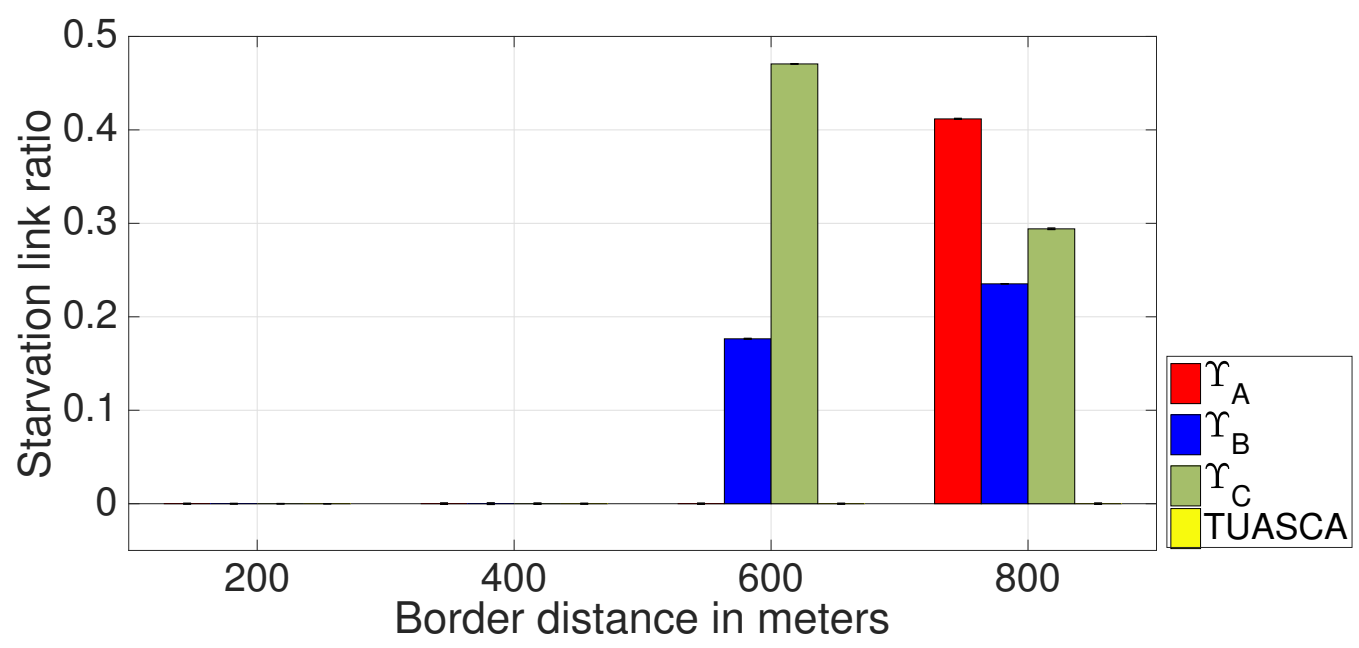

Figure 4.9: The comparison of starvation link ratio using three channels in random topologies 
In Figures 4.8 and 4.9 , when the border distance is less than $R_{c s}$ (i.e. $200 \mathrm{~m}$ and $400 \mathrm{~m}$, the effective carrier sensing scenarios), the starvation link ratios of all CA algorithms are zero, which means starvation does not exist. When the border distance is greater than $R_{c S}$ (i.e. between $600 \mathrm{~m}$ and $800 \mathrm{~m}$, the ineffective carrier sensing scenarios), only the TUASCA algorithm remains the starvation link ratio as zero while flow starvation exists in the channel allocation of other CA algorithms. In the case that random network size is $600 \mathrm{~m}$ in Figure 4.9 , the channel allocation of the clique-based CA algorithm using interference set selection $\gamma_{A}$ experiences no starvation. It explains why the fairness index of the clique-based CA algorithm using interference set selection $\gamma_{A}$ is higher than the clique-based CA algorithm using interference sets $\gamma_{B}$ and $\gamma_{C}$ in $600 \mathrm{~m}$ (see Figure 4.7).

The zero starvation link ratio of TUASCA algorithm explains the corresponding high Jain's index in Figures 4.6 and 4.7. It also shows that preventing flow starvation contributes towards better fairness.

\subsubsection{Comparison of Highest-to-lowest Goodput Ratio}

The results in Figure 4.10 and 4.11 show the comparison of highest-tolowest goodput ratio with corresponding 95\% confidence interval among the clique-based CA algorithm using interference set selections $\gamma_{A}, \gamma_{B}$, and $\gamma_{C}$, and TUASCA algorithm in grid and random topologies respectively. In Figure 4.10 and 4.11 , the $X$ axis denotes the border distance in Figure 4.5 while the $\mathrm{Y}$ axis refers to the highest-to-lowest goodput ratio of different $C A$ algorithms.

In Figure 4.10 and 4.11 , when the border distance is less than $R_{c s}$ (i.e. $200 \mathrm{~m}$ and $400 \mathrm{~m}$, the effective carrier sensing scenarios), the highest-tolowest goodput ratios of all CA algorithms are very small. When the border distance is greater than $R_{c S}$ (i.e. between $600 \mathrm{~m}$ and $800 \mathrm{~m}$, the ineffective carrier sensing scenarios), the highest-to-lowest goodput ratio of the TUASCA algorithm remains very small compared with the other three algorithms. The small highest-to-lowest goodput ratio explains the high 


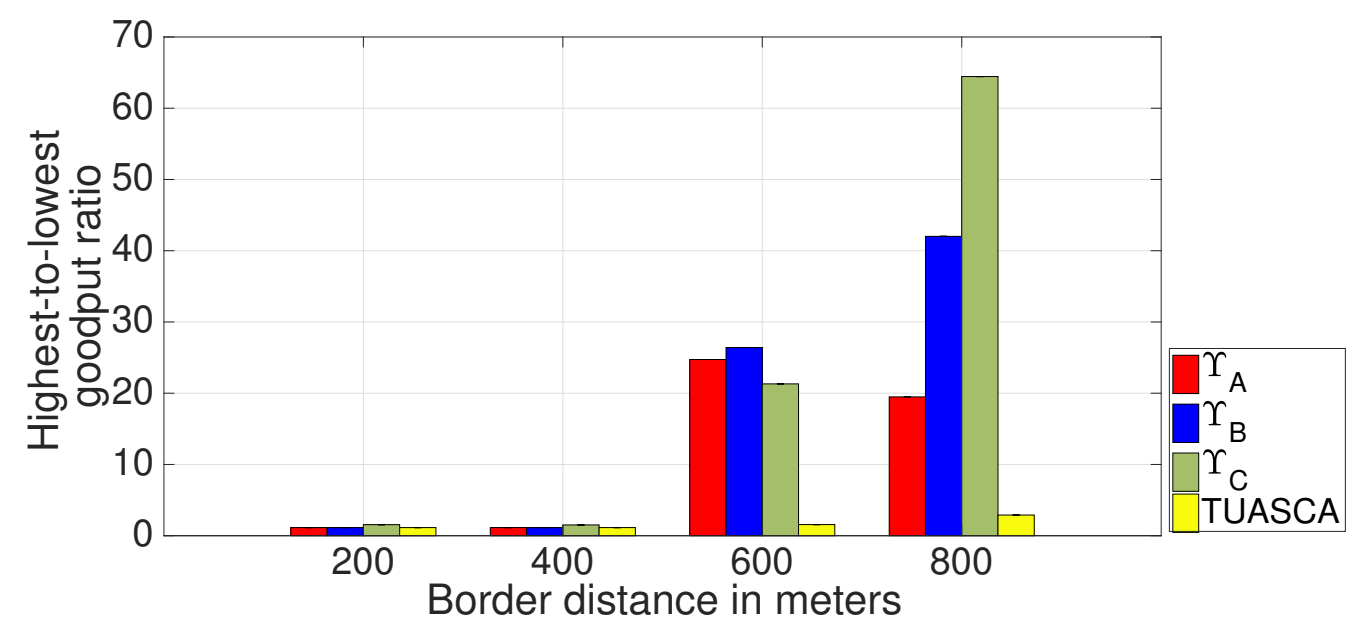

Figure 4.10: The comparison of highest-to-lowest goodput ratio using three channels in grid topologies

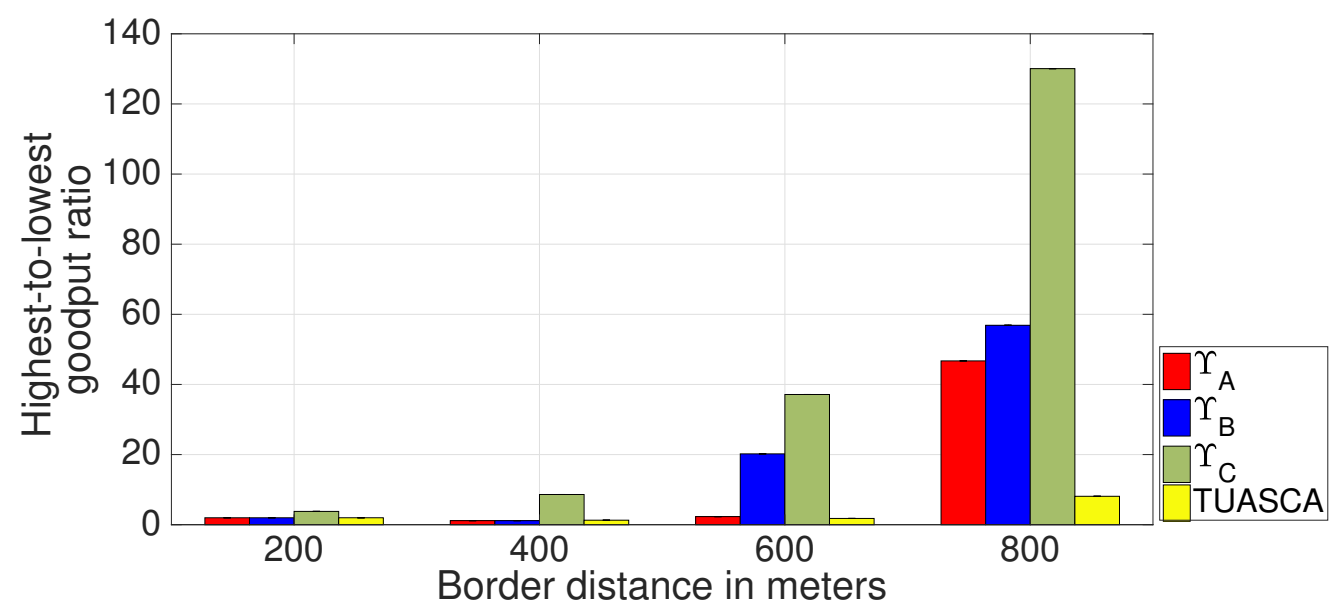

Figure 4.11: The comparison of highest-to-lowest goodput ratio using three channels in random topologies

Jain's index in Figures 4.6 and 4.7 


\subsubsection{Comparison of Average Goodput}

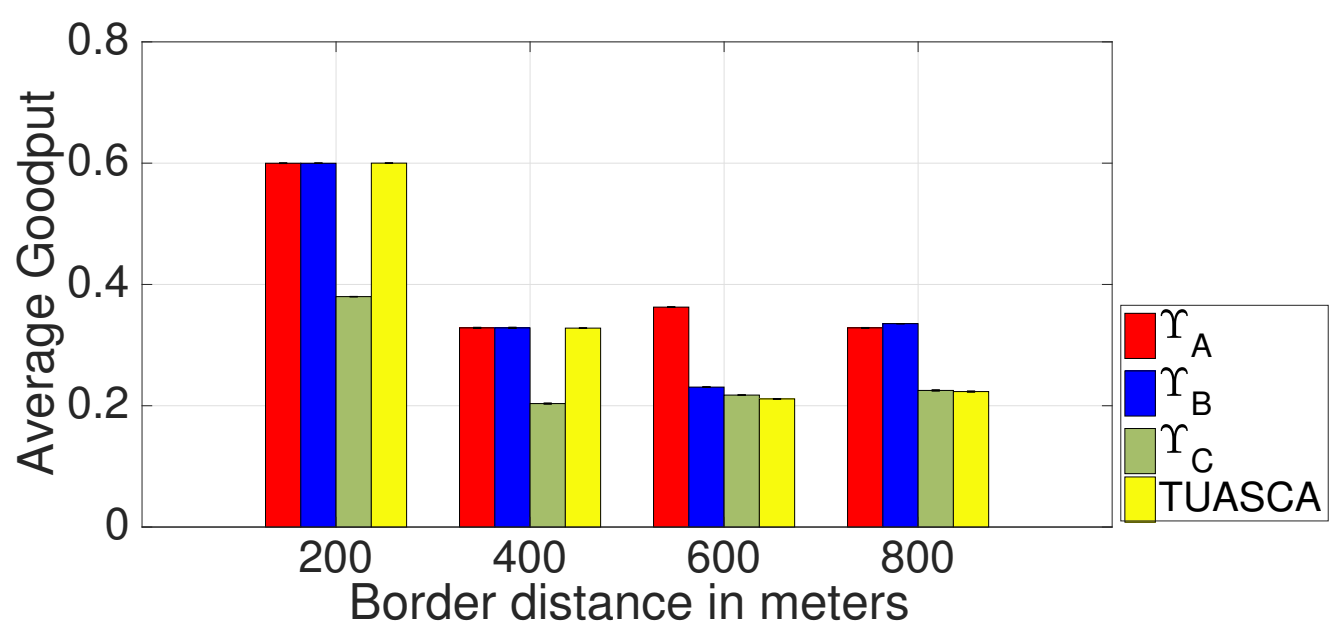

Figure 4.12: The comparison of average goodput using three channels in grid topologies

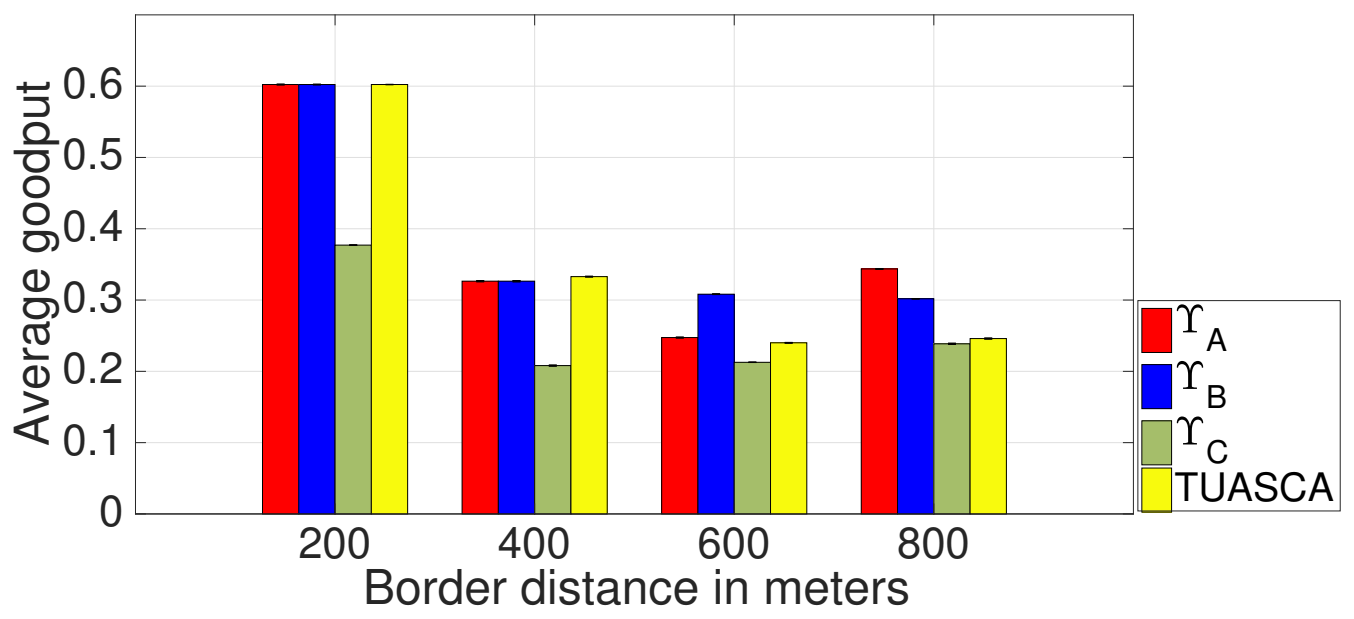

Figure 4.13: The comparison of average goodput using three channels in random topologies

To further evaluate the performance of the TUASCA algorithm, the 
TUASCA algorithm is compared with the other three CA algorithms in terms of average goodput. Figures 4.12 and 4.13 are the results of average goodput with corresponding $95 \%$ confidence interval from the cliquebased algorithm using interference set selections $\gamma_{A}, \gamma_{B}$, and $\gamma_{C}$, and TUASCA algorithm in grid and random topologies. In Figures 4.12 and 4.13 . the $\mathrm{X}$ axis denotes the border distance in Figure 4.5 while the $\mathrm{Y}$ axis refers to the average goodput of different CA algorithms.

In Figures 4.12 and 4.13 , when the border distance is less than $R_{C S}$ (i.e. $200 \mathrm{~m}$ and $400 \mathrm{~m}$, the effective carrier sensing scenarios), the TUASCA algorithm achieves the same average goodput as that of the clique-based CA algorithm using interference sets $\gamma_{A}$ and $\gamma_{B}$. When the border distance is greater than $R_{C S}$ (i.e. between $600 \mathrm{~m}$ and $800 \mathrm{~m}$, so called ineffective carrier sensing scenarios), the TUASCA algorithm decreases $3-42 \%$ in average goodput compared with the best result from the clique-based CA algorithm using interference set selection $\gamma_{A}$. The reason for the cliquebased CA algorithm using interference set selections $\gamma_{A}$ and $\gamma_{B}$ achieving higher goodput than TUASCA is that the clique-based algorithm using interference set selections $\gamma_{A}$ and $\gamma_{B}$ reuse all the channels in the ineffective carrier sensing scenarios. But the TUASCA algorithm partially reuses the channels to eliminate border effect and flow starvation that leads to a lower average goodput.

\subsubsection{Validation of Traffic-unaware Anti-starvation Chan- nel Assignment Algorithm with Twelve Channels}

In this subsection, the TUASCA algorithm is validated with the benchmarks using twelve channels through simulation. The example using twelve channel shows that flow starvation still exists in some scenarios even though the available channel is increased to 12 .

The grid topologies used in this subsection deploy links with $D=$ $1000 \mathrm{~m}, D_{t r}=50 \mathrm{~m}$ and constant interval $d$ of $30 \mathrm{~m}$ (see Figure 4.5). The 
simulation configurations is in Table 4.3. The number of available channels is twelve and the carrier sensing range is $675 \mathrm{~m}$ according to the configuration in Table 4.3. All averages of simulation results shown are reported with confidence interval of $95 \%$ with the range from $[3.6,53.1] \mathrm{kbps}$ under the assumption that the averages are normally distributed.

The TUASCA algorithm is validated from four aspects, overall Jain's index, starvation link ratio, highest-to-lowest goodput ratio and average goodput.

Table 4.3: Simulation Parameters

\begin{tabular}{l|l}
\hline Parameter Name & Value \\
\hline Transmission Power & $20 \mathrm{dBm}$ \\
\hline Receiver Sensitivity & $-85 \mathrm{dBm}$ \\
\hline Path Loss Model & Two-Ray \\
\hline Shadowing and Fading Model & None \\
\hline MAC Layer & PCS \\
\hline Routing & Static Routing \\
\hline Transportation Layer & UDP \\
\hline Physical Layer & IEEE $802.11 \mathrm{a}$ \\
\hline Data Rate & $6 \mathrm{Mbps}$ \\
\hline Packet Size & $1500 \mathrm{Bytes}$ \\
\hline Inter-packet Interval & $2 \mathrm{~ms}$ \\
\hline
\end{tabular}

Figures 4.14 to 4.17 show the results of Jain's index, starvation link ratio, highest-to-lowest goodput ratio, average goodput with corresponding 95\% confidence interval in the grid topology ( $D=1000 \mathrm{~m}$ in Figure 4.5).

In Figure 4.14, the TUASCA algorithm achieves the highest Jain's index among all CA algorithms. Even though using twelve channels in such a dense topology, the clique-based CA algorithm using interference sets $\gamma_{A}$, $\gamma_{B}$, and $\gamma_{C}$ still experience flow starvation but TUASCA algorithm effectively prevents flow starvation (see Figure 4.15). This shows that existing 


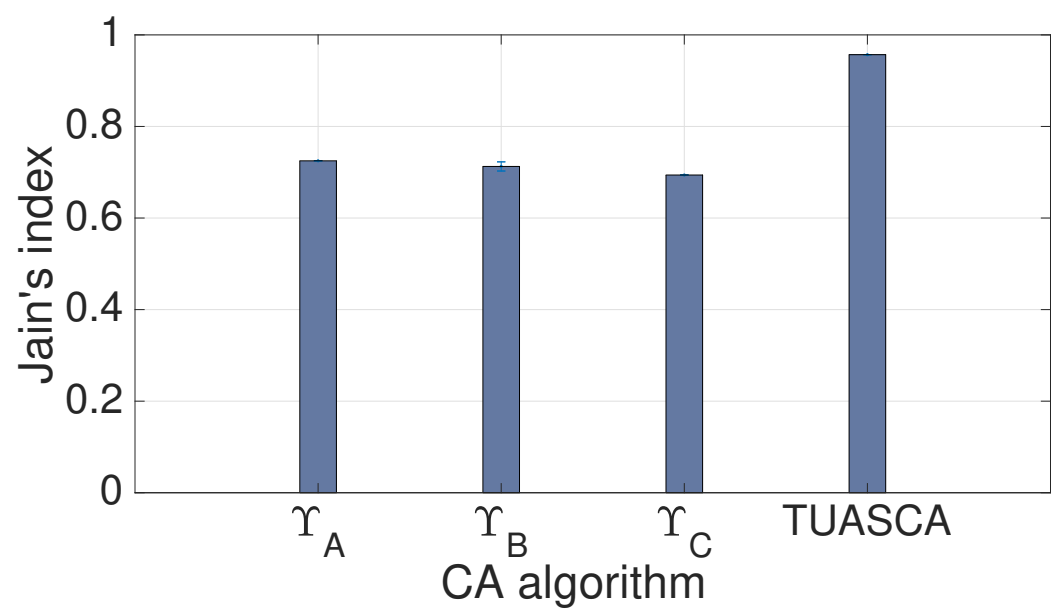

Figure 4.14: The comparison of Jain's index using twelve channels in a grid topology

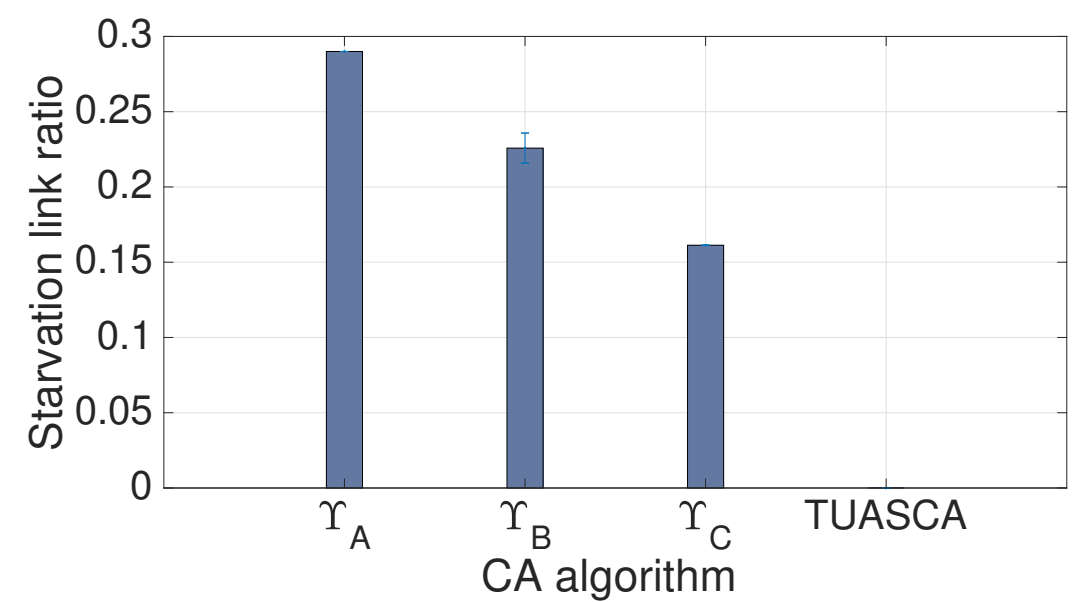

Figure 4.15: The comparison of starvation link ratio using twelve channels in a grid topology

interference models still drive CA algorithm to flow starvation and poor fairness with more channels available because they only consider local interference. 


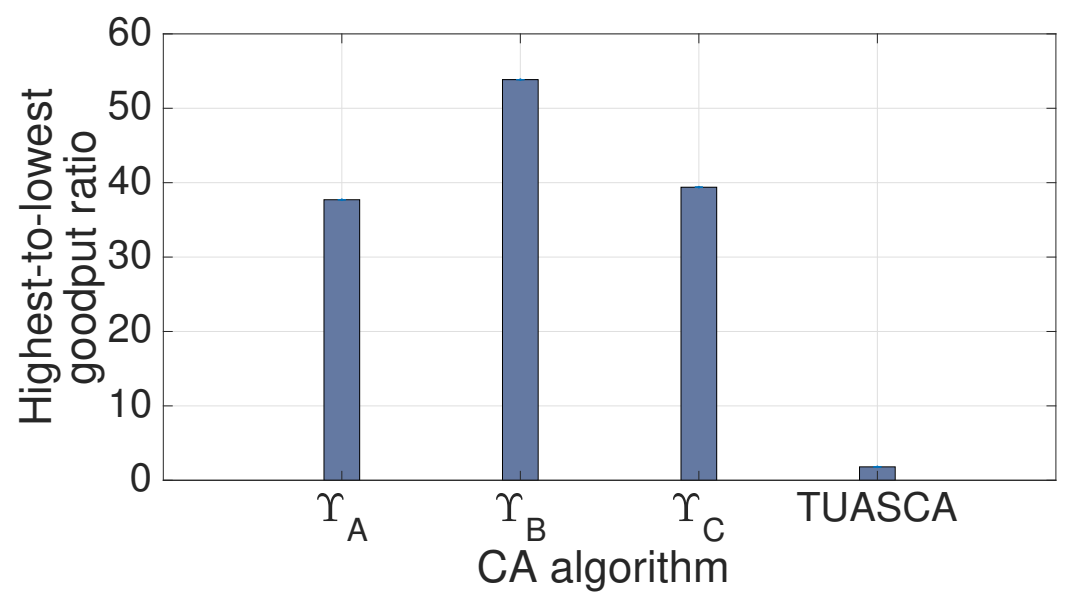

Figure 4.16: The comparison of highest-to-lowest goodput ratio using twelve channels in a grid topology

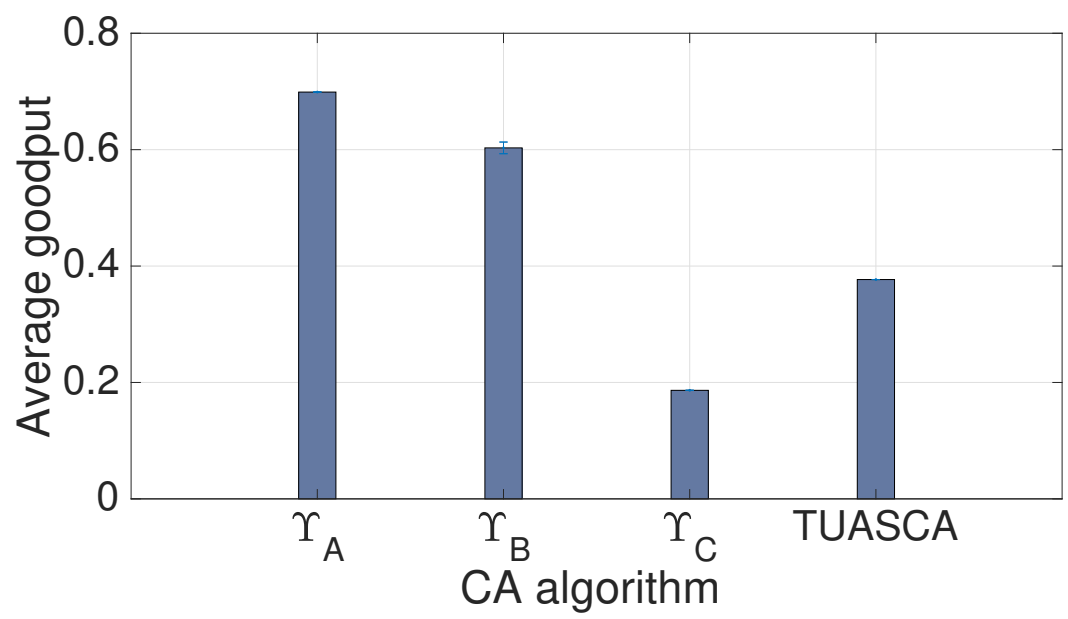

Figure 4.17: The comparison of average goodput using twelve channels in a grid topology

Figure 4.16 shows that the TUASCA algorithm outperforms the other CA algorithms in terms of highest-to-lowest goodput ratio. This matches with the trend in Jain's index in Figure 4.14. For average goodput, the 
TUASCA algorithm achieves more than the clique-based algorithm using interference set selection $\gamma_{C}$ but the clique-based algorithm using interference sets $\gamma_{A}$ and $\gamma_{B}$ achieves higher goodput than TUASCA. Along the same lines of explanation in Section 4.2.3.5 the clique-based algorithm using interference set selections $\gamma_{A}$ and $\gamma_{B}$ fully reuse the twelve available channels for the links in the topology but the TUASCA algorithm partially reuse these channels to separate border sets and middle sets with different channels that sacrifices average goodput. The interference set selection $\gamma_{C}$ utilises fewer channels due to its selection strategy.

\subsubsection{Discussion About Traffic-unaware Anti-starvation Chan- nel Assignment Algorithm}

Through simulation validation, the TUASCA algorithm achieves overall best fairness compared with the clique-based CA algorithm using three interference set selections. In both effective and ineffective carrier sensing scenarios, the TUASCA algorithm yields high Jain's index, "zero" starvation link ratio, and low highest-to-lowest goodput ratio with 3 and 12 available channels. Even with 12 available channels, the channel allocation from the clique-based CA algorithm still leads to flow starvation and poor fairness. It further proves the conclusion in Section 4.1 that the interference model used in CA algorithm needs to consider global interference for preventing flow starvation and improving fairness.

However, the TUASCA algorithm attains lower average goodput compared with the clique-based CA algorithm using interference set selections $\gamma_{A}$ and $\gamma_{B}$. To prevent flow starvation and improve fairness, the TUASCA algorithm partitions the border sets and middle-link set with different channel to improve fairness, sacrificing spatial reuse and yields lower average goodput.

Next section will explain how to extend the TUASCA algorithm to resolve flow starvation and improve fairness with unsaturated traffic de- 
mand.

\subsection{A Traffic-aware Anti-starvation Channel As- signment Algorithm for Improving Fairness}

This section explains the development of a Traffic-Aware Anti-Starvation Channel Assignment (TAASCA) algorithm to resolve flow starvation and improve the fairness among the links with consideration of traffic demand. The TAASCA algorithm is improving fairness by explicitly considering traffic demand in IEEE 802.11 WBN planning. The proposed traffic-aware anti-starvation CA (TAASCA) algorithm builds upon the TUASCA algorithm in Section 4.2 .

The next sections introduce the design of this TAASCA algorithm and validate TAASCA through simulation.

\subsubsection{The Design of Traffic-aware Anti-starvation Channel Assignment Algorithm}

The TAASCA algorithm considers traffic demand and the inputs are link set $E$, node position $P$, available channel number $m$, and traffic demand $f$. In addition, fixed carrier sensing range $R_{C S}$ is given. The output of TAASCA is the channel allocation of all links.

The TAASCA algorithm follows the same logic as that in the TUASCA algorithm to prevent flow starvation and improve fairness (see Figure 4.18). However, with a new input of traffic demand, the TAASCA algorithm has two main changes on the TUASCA algorithm. One is the global interference condition. Another change in the TAASCA algorithm is the capacity condition.

Firstly, the TAASCA algorithm calculates the border distance $D$ and checks whether $D$ is greater than the carrier sensing range $R_{C S}$. For an effective carrier sensing scenario $\left(D \leq R_{C S}\right)$, the TAASCA algorithm al- 


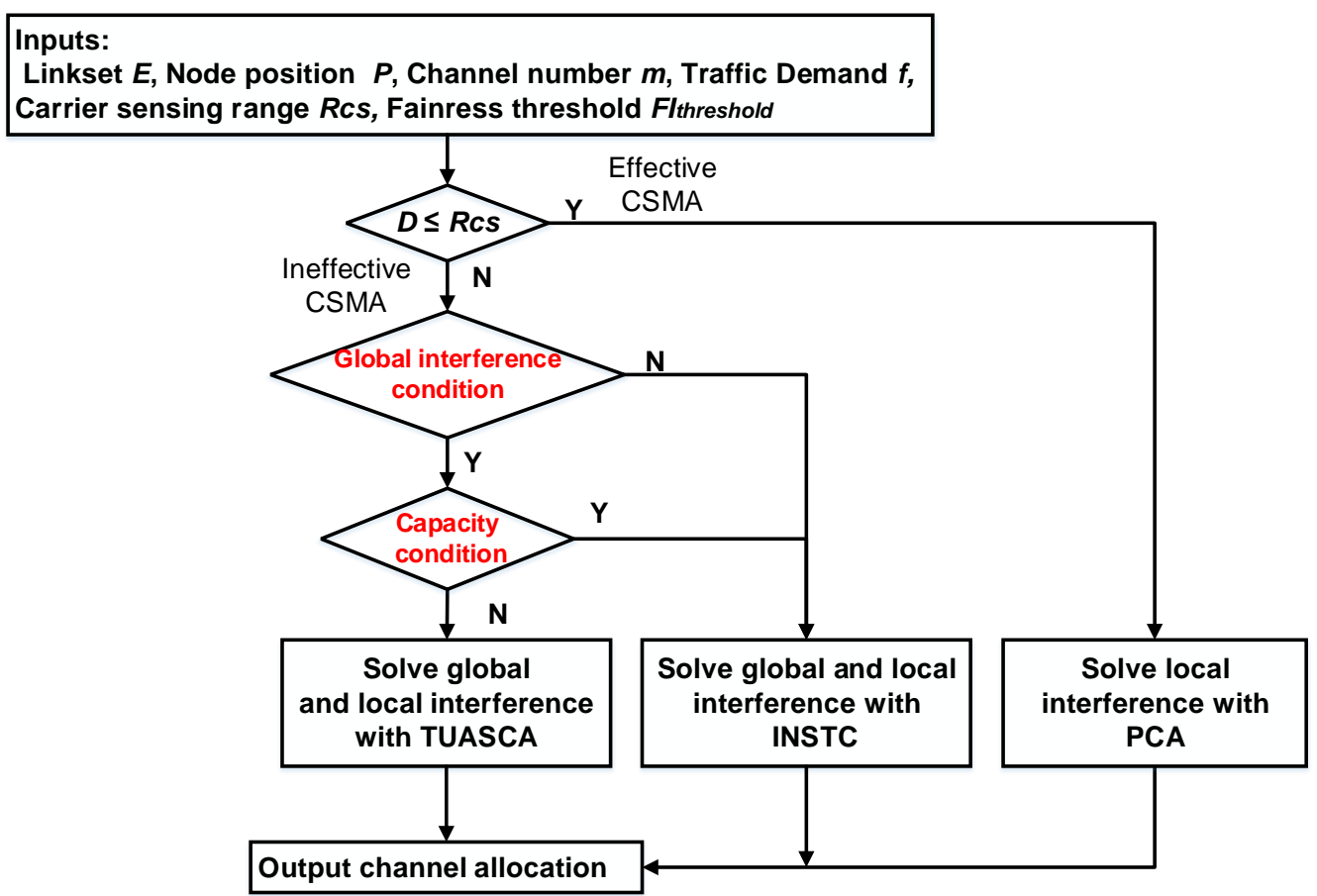

Figure 4.18: The logic flow of the TAASCA algorithm

leviates local interference without spatial reuse by using a partition CA algorithm. The purpose of using a partition CA algorithm is to alleviate interference and achieve the fairness among subgroups using different channels.

For an ineffective carrier sensing scenario $\left(D>R_{C S}\right)$, the TAASCA algorithm will first check the global interference condition to identify the potential existence of global interference (border effect). Under unsaturated traffic assumption, the existence of global interference depends on the global interference condition (see Section 4.1). The global interference condition is defined as: $\sum_{j \in\left\{G I S_{L B} \cap G I S_{R B}\right\}} f(j) \geq 1$, where $G I S_{L B}$ and $G I S_{R B}$ are the global left border set and right border set in the given IEEE 802.11 WBN, $f(j)$ is the normalised traffic demand of link $j$ with respect to the maximum net capacity. 
If the constraint $\sum_{j \in\left\{G I S_{L B} \cap G I S_{R B}\right\}} f(j) \geq 1$ holds, global interference potentially exists. The TAASCA algorithm checks whether the capacity condition is sufficient to prevent flow starvation with spatial reuse. Different from using the link number in the TUASCA algorithm, the TAASCA algorithm expresses the capacity condition as: $\sum_{j \in\{B \cap \gamma(B)\}} f(j) \leq m$, where $\gamma(B)$ is the local interference set of a border link $B$ and $m$ refers to the normalised capacity of all available channels, $f(j)$ is the normalised traffic demand of link $j$ with respect of the maximum net capacity.

If the constraint $\sum_{j \in\{B \cap \gamma(B)\}} f(j) \leq m$ holds, the available channels are sufficient to directly address the potential global interference with spatial reuse. The TAASCA algorithm solves global and local interference with spatial reuse. If $\sum_{j \in\{B \cap \gamma(B)\}} f(j) \leq m$ does not hold, the available channel capacity is not sufficient to resolve global interference with spatial reuse. The TAASCA algorithm invokes the TUASCA algorithm to solve for both global and local interference. The TUASCA algorithm solves global interference by partitioning the global border sets and middle-link set with different channels and allocates identical channel to $G I S_{L B}$ and $G I S_{R B}$ to improve spatial reuse.

The outline of TAASCA is listed in Algorithm 6. The structure of TAASCA is the same as that of TUASCA. The difference between them is that the TAASCA algorithm change the condition expressions in line 10 and line 16 according to new global interference and capacity conditions.

\subsubsection{Benchmark For Traffic-aware Anti-starvation Chan- nel Assignment Algorithm}

The benchmark algorithm for the TAASCA algorithm is selected with the balanced static CA (BSCA) algorithm [87] discussed in Section 2.4.3 on page 33 (see Algorithm 7 ) with three different interference set selections $\gamma_{A}, \gamma_{B}$, and $\gamma_{C}$ (see Section 4.2.2). The BSCA algorithm represents the traffic-aware channel assignment with consideration of fairness that is sim- 


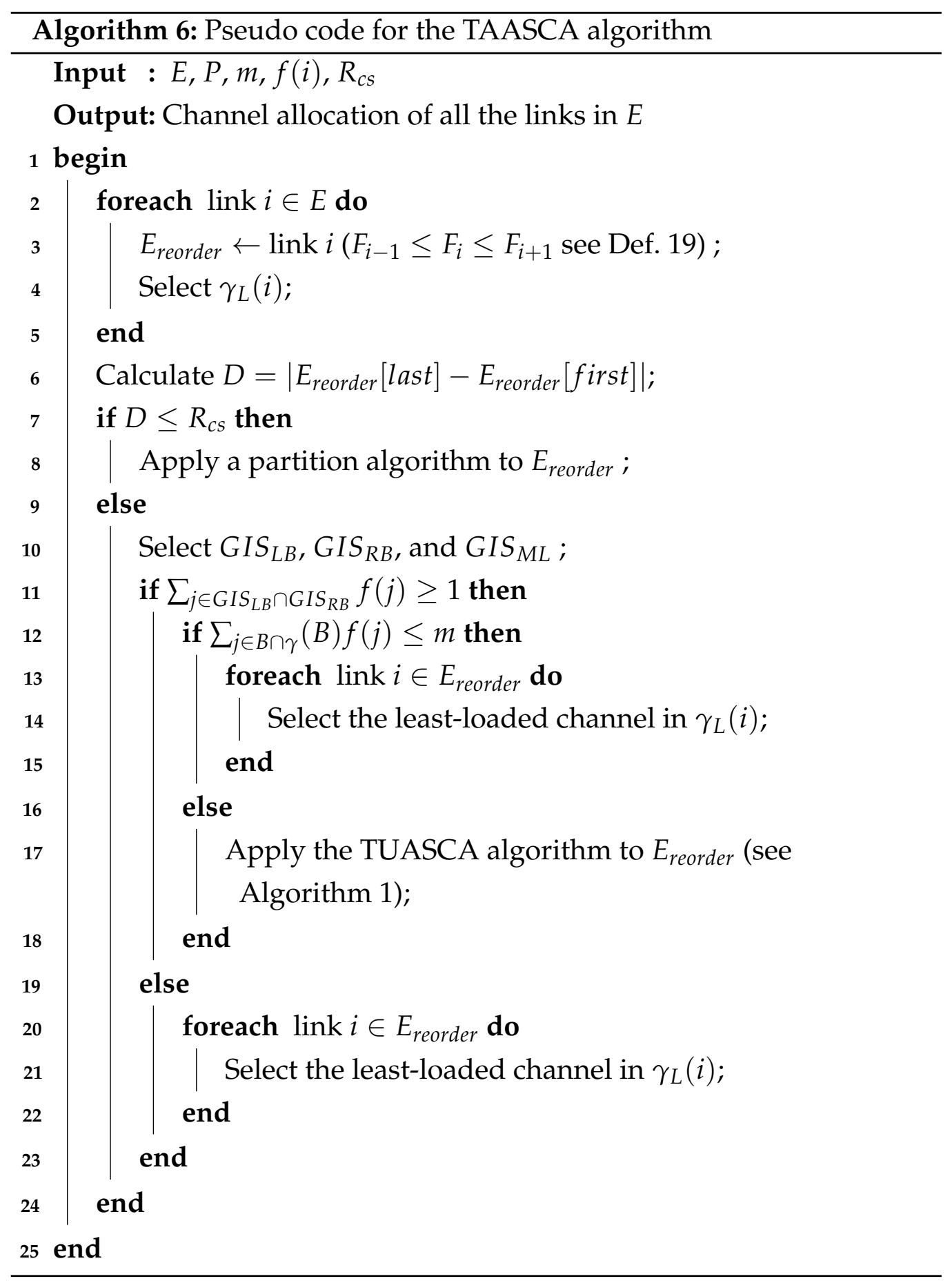


ilar to RCL [121] (more details in Section 2.4.3 on page 33). In this thesis, we only borrow the basic strategy of channel assignment in [87] for minimising interference to simplify the problem.

The BSCA algorithm used in this thesis has the same inputs and outputs as TAASCA. The logic of BSCA algorithm is listed in Algorithm 7. The difference between the BSCA and the clique-based INSTC CA algorithms in Section 4.2.2 is that they use different allocation strategy. The clique-based INSTC CA algorithm allocates the least used channel to each link that is based on calculating the total number of interfering links in the interference sets. The BSCA algorithm allocates the least traffic-load channel to each link that is based on calculating the sum of the traffic demands of interfering links in the interference sets.

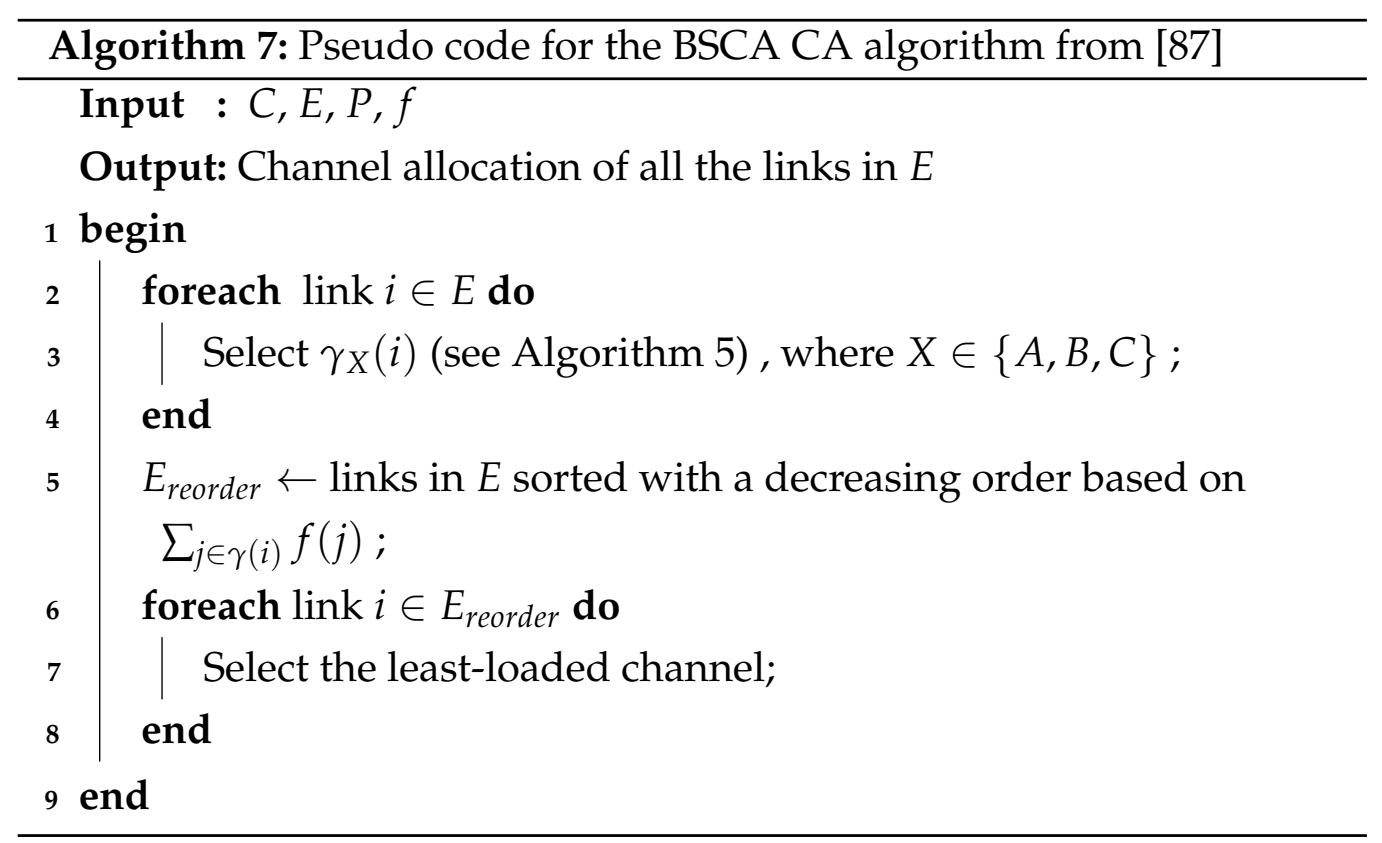




\subsubsection{Validation of Traffic-aware Anti-starvation Channel Assignment Algorithm Using Three Channels}

The inputs of the TAASCA and BSCA algorithms and simulation configurations are the same as those in Section 4.2.3.1. Four traffic demands are selected as 0.4 and 0.6 representing medium traffic, and 0.8 and 1.0 representing heavy traffic. These four traffic demands are the normalised traffic demands with respect to the maximum net capacity.

All averages of simulation results shown are reported with confidence interval of $95 \%$ with the range from $[5.2,15.66] \mathrm{kbps}$ under the assumption that the averages are normally distributed.

Since flow starvation is not present in the effective carrier sensing scenarios, the results are listed from an ineffective carrier sensing scenario ( $D=800 \mathrm{~m}, d=50 \mathrm{~m}, D_{t r}=50 \mathrm{~m}$ in Figure 4.5) using three channels as the examples. The TAASCA algorithm is validated from four aspects, overall Jain's index, starvation link ratio, highest-to-lowest goodput ratio and average goodput (see the definitions in Section 4.2.3.1).

\subsubsection{Comparison of Jain's Index}

Figures 4.19 and 4.20 show the comparison of Jain's index with corresponding 95\% confidence interval using three channels among different CA algorithms in grid and random topologies respectively. In Figures 4.19 and 4.20, the $\mathrm{X}$ axis denotes the normalised traffic demand with respect to the maximum net capacity while the $Y$ axis refers to the achieved Jain's index of the channel allocations from different algorithms.

In Figures 4.19 and 4.20 , the TAASCA algorithm achieves the highest Jain's index with four traffic demands. The Jain's index of the BSCA algorithm using interference sets $\gamma_{A}$ and $\gamma_{B}$ increases when the traffic demand decreases. It is because the global interference reduces when the traffic demands of the border links decrease. However, the BSCA algorithm using interference $\gamma_{C}$ yields low Jain's index for heavy and medium traffic 


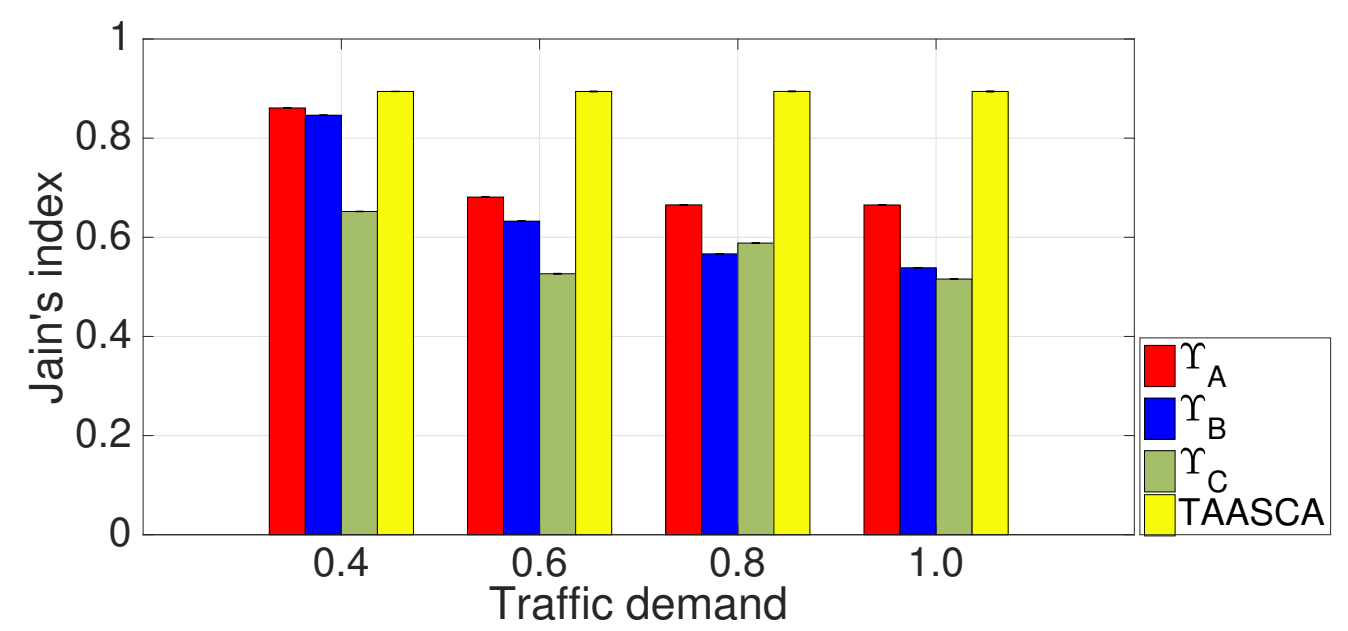

Figure 4.19: The comparison of Jain's index using three channels in a grid topology

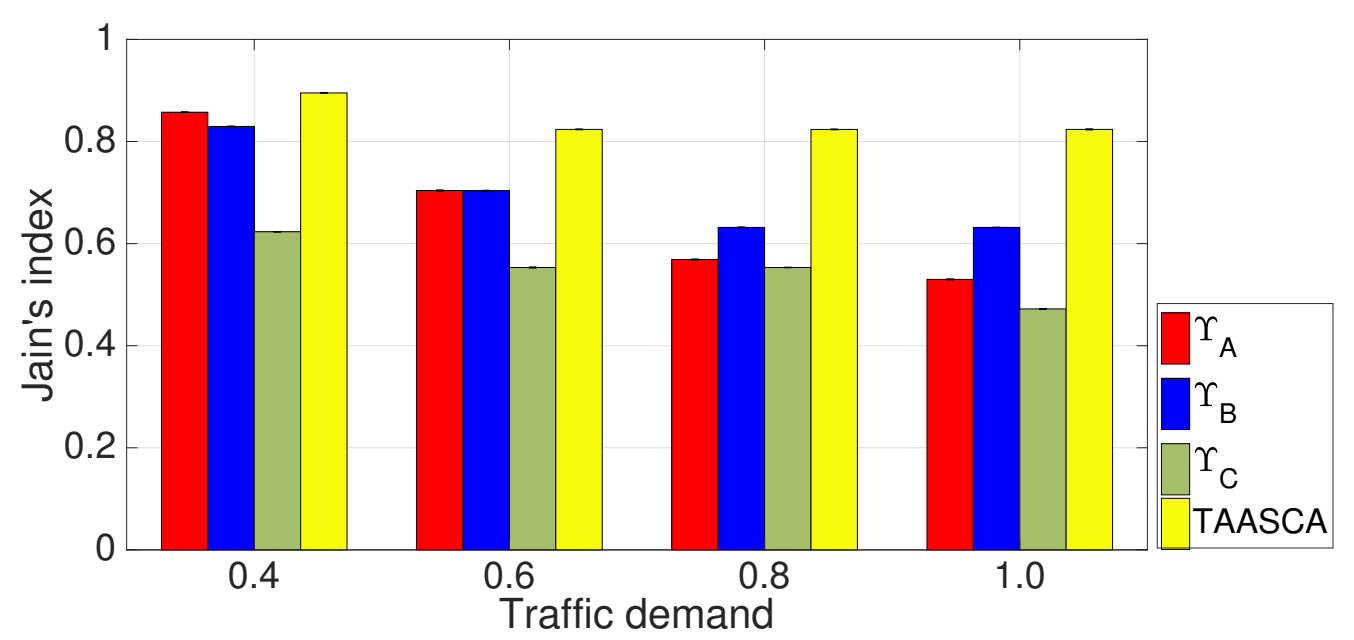

Figure 4.20: The comparison of Jain's index using three channels in a random topology

demands because they did not fully utilise the three available channels. 


\subsubsection{Comparison of Starvation Link Ratio}

Figures 4.21 and 4.22 show the results of starvation link ratio with corresponding 95\% confidence interval among four CA algorithms using 3 channels in grid and random topologies respectively. In Figures 4.21 and 4.22 , the $\mathrm{X}$ axis denotes the normalised traffic demand while the $\mathrm{Y}$ axis refers to the starvation link ratio of different $C A$ algorithms.

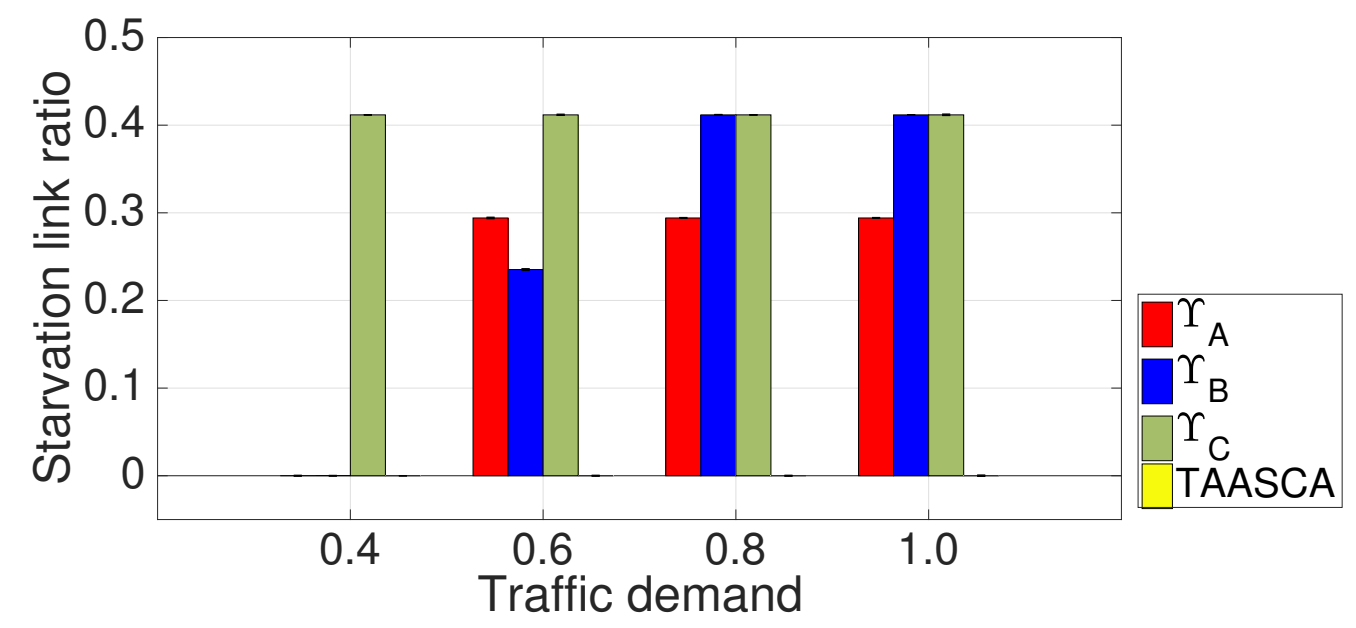

Figure 4.21: The comparison of starvation link ratio using three channels in a grid topology

In Figures 4.21 and 4.22 , the TAASCA algorithm achieves "zero" starvation link ratio with four traffic demands. As the TAASCA algorithm resolves the global interference, flow starvation does not exist that explains the high Jain's index in Figures 4.19 and 4.20 . The flow starvation ratio of the the BSCA algorithm using interference sets $\gamma_{A}$ and $\gamma_{B}$ has a decreasing pattern with the decrease of the traffic demand. That is why their Jain's index increases with the decrease of the traffic demand. The BSCA algorithm using interference set selection $\gamma_{C}$ remains a constant starvation link ratio with four traffic demands. It implies that flow starvation does not reduce in these two channel allocations when the traffic demands decrease. 


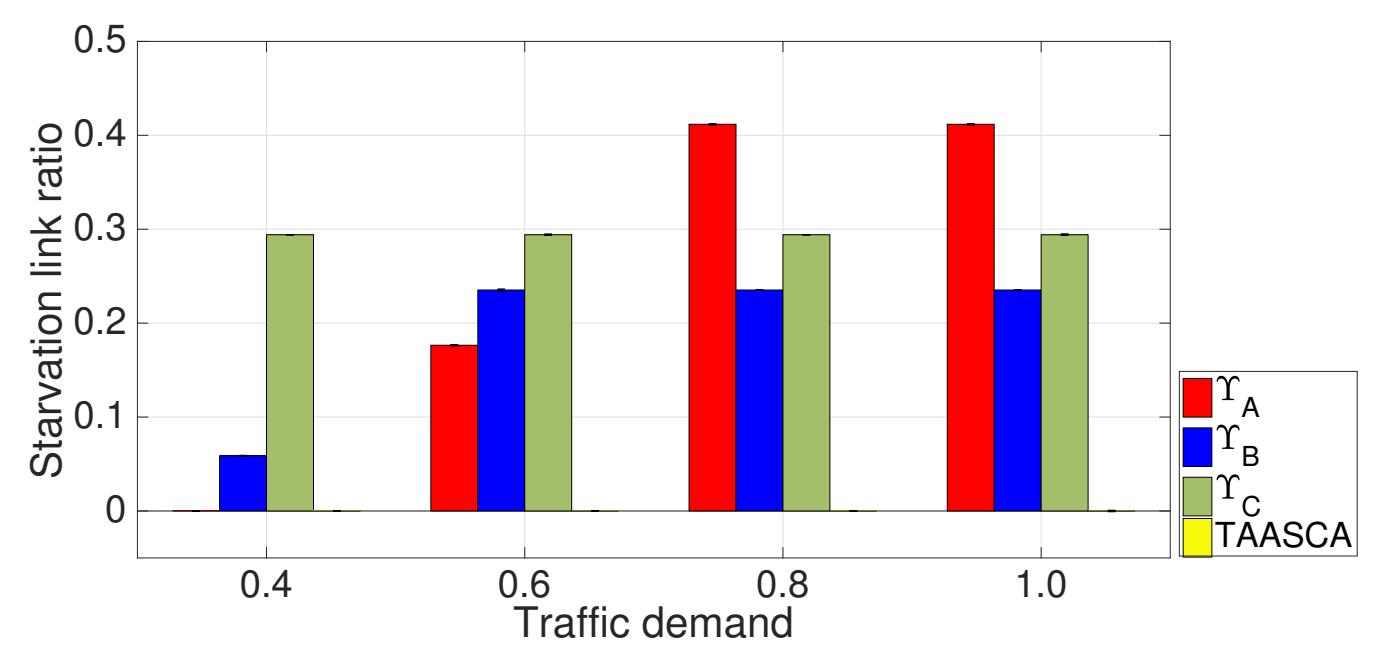

Figure 4.22: The comparison of starvation link ratio using three channels in a random topology

\subsubsection{Comparison of Highest-to-lowest Goodput Ratio}

Figures 4.23 and 4.24 show the comparison of highest-to-lowest goodput ratio with corresponding 95\% confidence interval among four CA algorithms using three channels in grid and random topologies respectively. In Figures 4.23 and 4.24 , the $X$ axis denotes the normalised traffic demand while the $Y$ axis refers to the highest-to-lowest goodput ratio of different CA algorithms.

In Figures 4.23 and 4.24 , the TAASCA algorithm remain low highestto-lowest goodput ratios with four traffic demands. That matches with the high Jain's index in Figures 4.19 and 4.20. The highest-to-lowest goodput ratios of the BSCA algorithm using interference sets $\gamma_{A}, \gamma_{B}$, and $\gamma_{C}$ have a decreasing pattern with the decrease of the traffic demand. That also matches with their Jain's index patterns. 


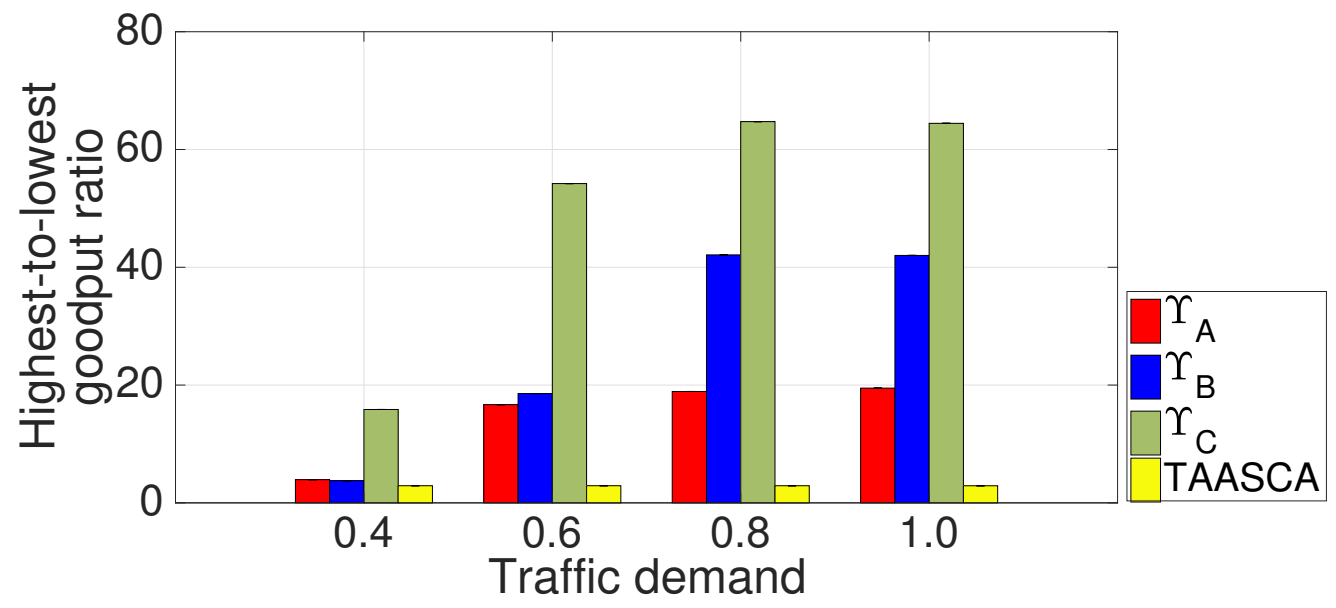

Figure 4.23: The comparison of highest-to-lowest goodput ratio using three channels in a grid topology

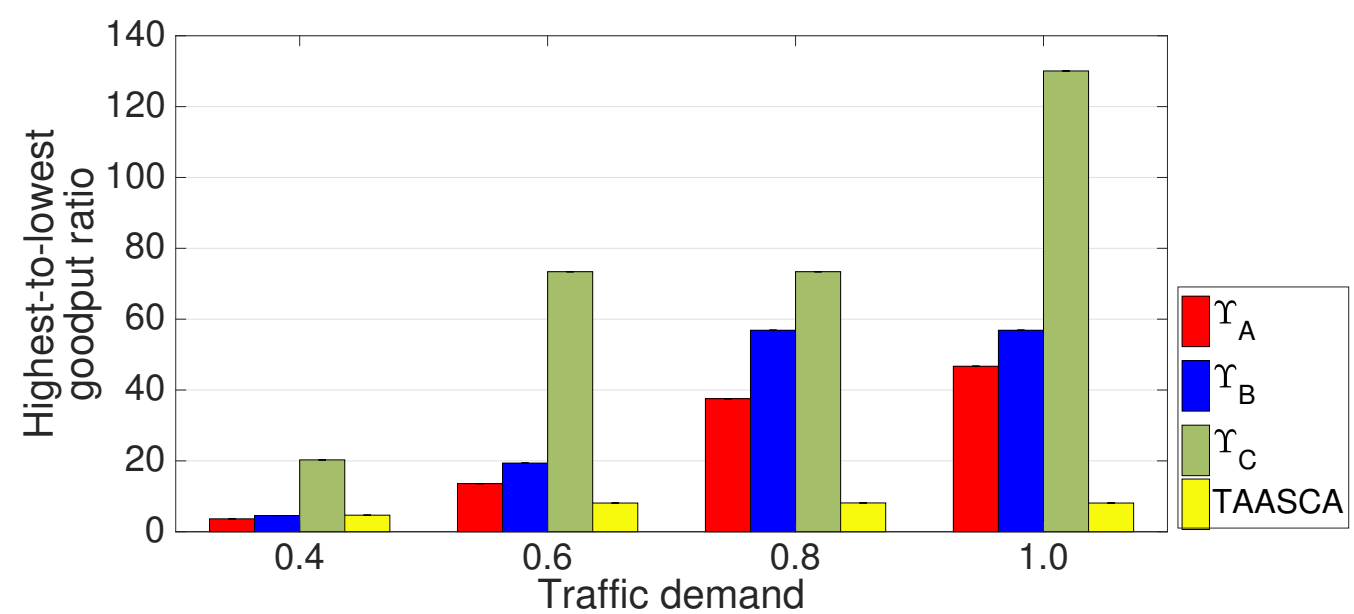

Figure 4.24: The comparison of highest-to-lowest goodput ratio using three channels in a random topology

\subsubsection{Comparison of Average Goodput}

Figures 4.25 and 4.26 show the average goodput with corresponding 95\% confidence interval from four CA algorithms using three channels in grid 
and random topologies respectively. In Figures 4.25 and 4.26 , the $X$ axis denotes the normalised traffic demand while the $Y$ axis refers to the normalised average goodput from different $C A$ algorithms with respect to the maximum net capacity.

In Figures 4.25 and 4.26 , the average goodput of the TAASCA algorithm is less than those of the BSCA algorithm with $\gamma_{A}$ and $\gamma_{B}$. The same as the TUASCA algorithm, the TAASCA algorithm resolves the global interference by partitioning the border sets and middle link set with different channels that sacrifices a part of spatial reuse. The BSCA algorithm with $\gamma_{A}$ and $\gamma_{B}$ fully reuse the spatial resource. The BSCA algorithm with $\gamma_{C}$ does not utilise the whole available channels and yields the lowest average goodput.

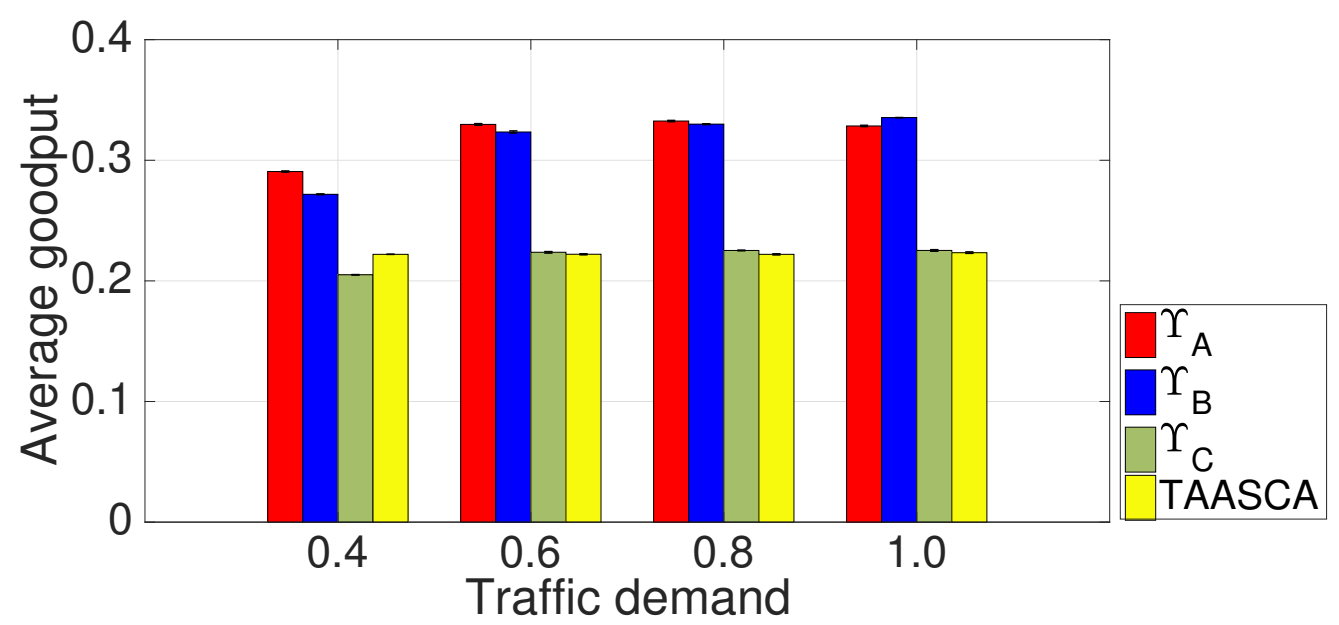

Figure 4.25: The comparison of average goodput using three channels in a grid topology 


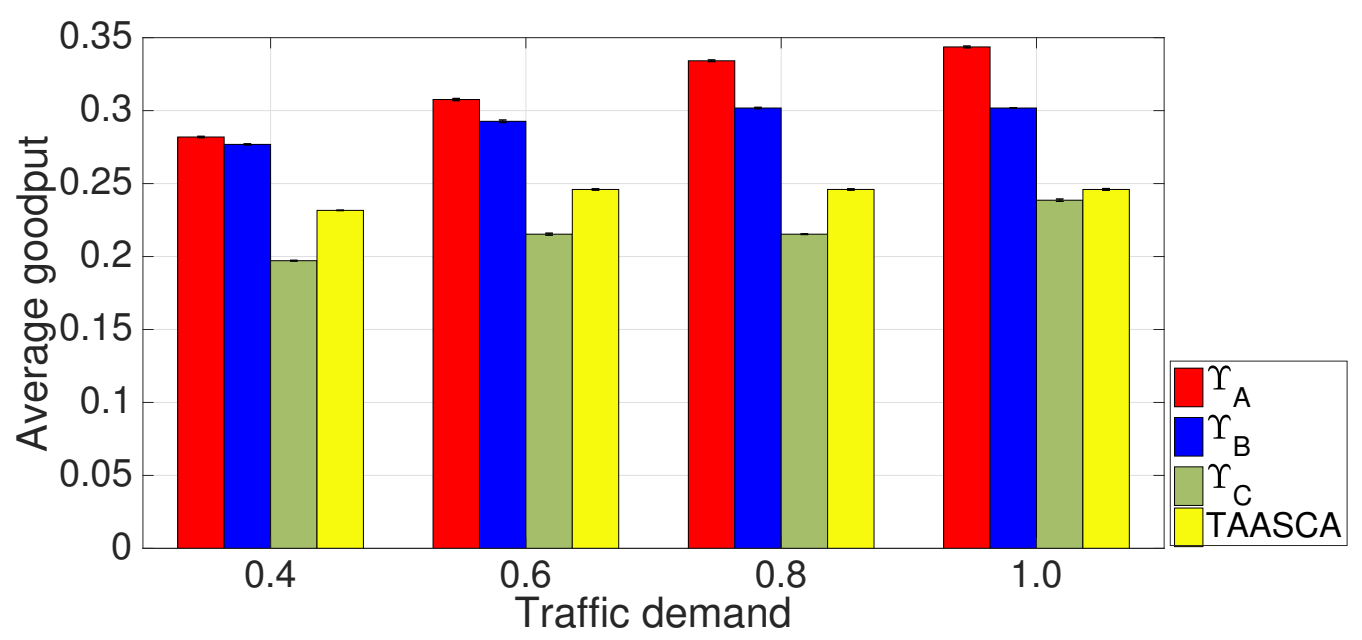

Figure 4.26: The comparison of average goodput using three channels in a random topology

\subsubsection{Validation of Traffic-aware Anti-starvation Channel Assignment Algorithm Using Twelve Channels}

In this subsection, the TAASCA algorithm is validated with the benchmark using twelve channels through simulation. The inputs and simulation configurations are the same as those in section 4.2.4. With more available channels, four CA algorithms are expected to be more effective on preventing flow starvation and fairness than using less channels. The grid topology used in this subsection deploy links with $D=1000 \mathrm{~m}, D_{t r}=50 \mathrm{~m}$ and constant interval $d$ of $30 \mathrm{~m}$ (see Figure 4.5).

The simulation parameters are listed in Table 4.3 . The number of available channels is twelve and the carrier sensing range is $675 \mathrm{~m}$ according to the configuration in Table 4.3. All averages of simulation results shown are reported with confidence interval of $95 \%$ with the range from $[3.6,53.1] \mathrm{kbps}$ under the assumption that the averages are normally distributed.

The TAASCA algorithm is validated from four aspects, overall Jain's 
index, starvation link ratio, highest-to-lowest goodput ratio and average goodput.

Figures 4.27 to 4.30 show the results of Jain's index, starvation link ratio, highest-to-lowest goodput ratio, average goodput with corresponding 95\% confidence interval in the grid topology ( $D=1000 \mathrm{~m}$ in Figure 4.5).

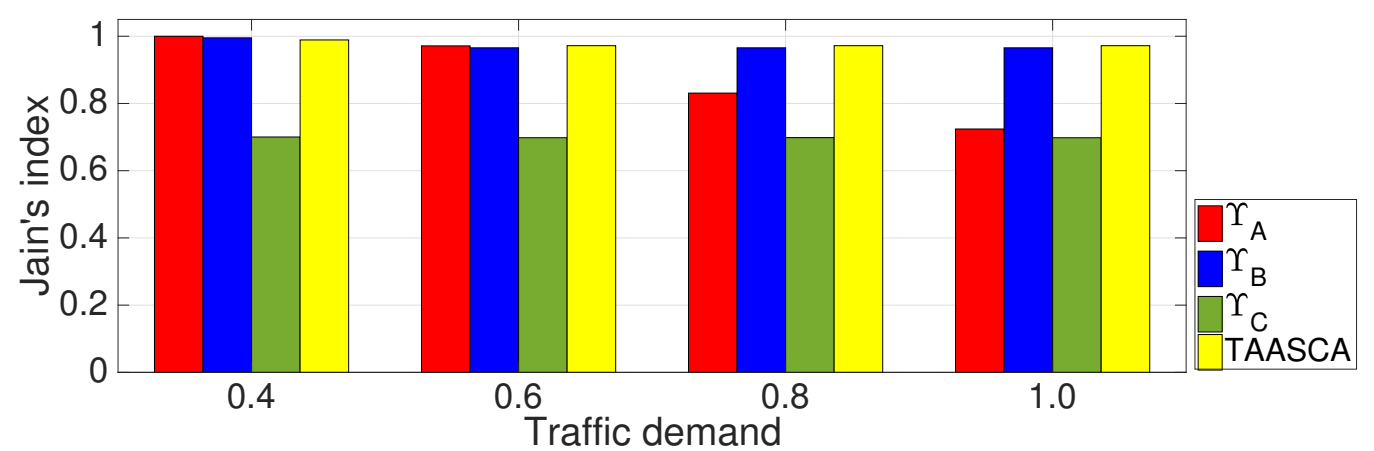

Figure 4.27: The comparison of fairness index using twelve channels in a grid topology

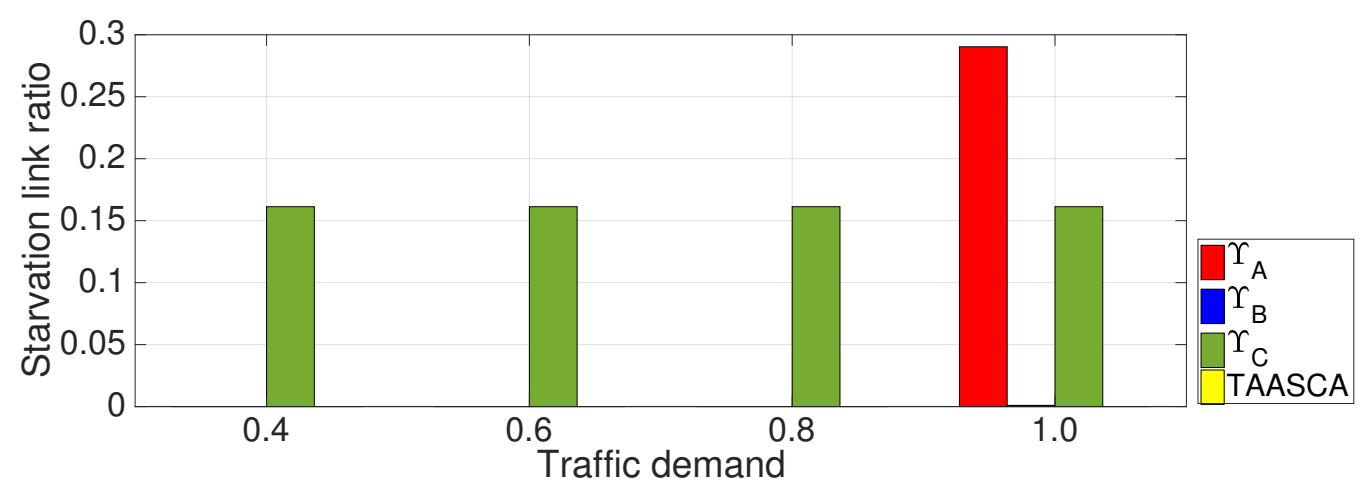

Figure 4.28: The comparison of starvation ratio using twelve channels in a grid topology

In Figure 4.27, the TAASCA algorithm maintains a high Jain's index for four different traffic demands. Even though using twelve channels, 
the BSCA algorithm using interference set selections $\gamma_{A}$ and $\gamma_{C}$ still lead to flow starvation in such a dense topology. The TAASCA and BSCA algorithms using interference set selection $\gamma_{B}$ effectively prevent flow starvation (see Figure 4.28). This shows that existing interference interference set selections $\gamma_{A}$ and $\gamma_{C}$ still drive CA algorithm to flow starvation and poor fairness with more channels available because they only consider local interference.

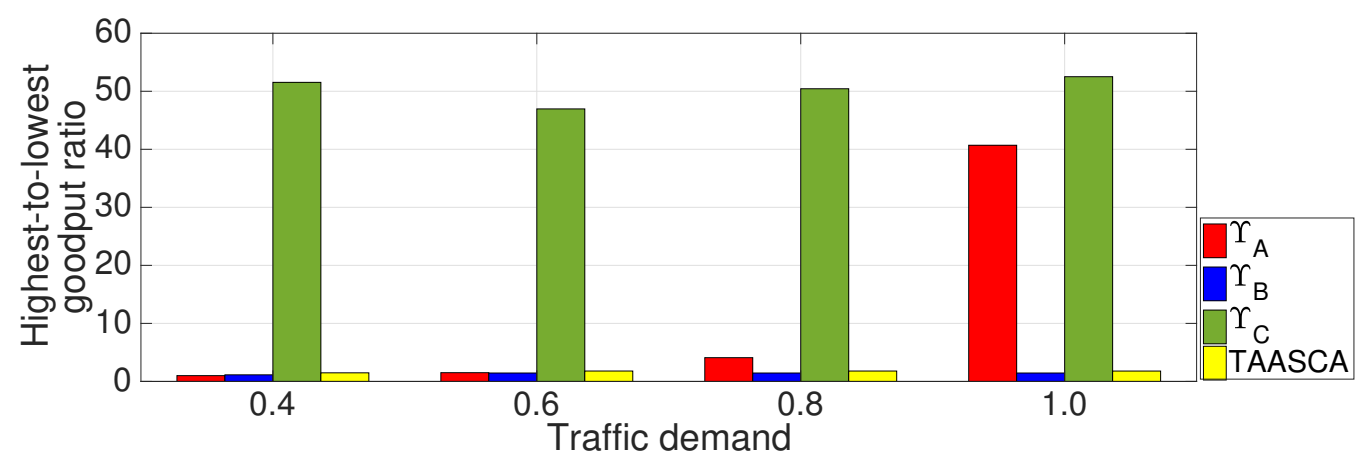

Figure 4.29: The comparison of high-to-low goodput ratio using twelve channels in a grid topology

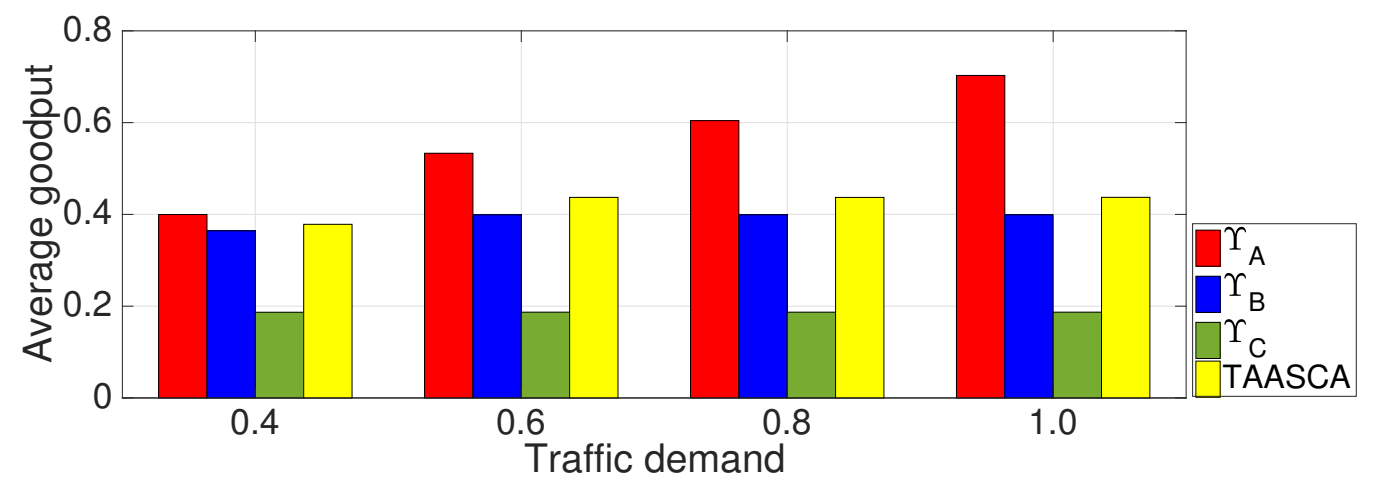

Figure 4.30: The comparison of average goodput using twelve channels in a grid topology 
In Figure 4.29, the result shows that the TAASCA algorithm and the BSCA algorithm using interference set selection $\gamma_{B}$ outperform the other CA algorithms in terms of highest-to-lowest goodput ratio. This matches with the trend in Jain's index in Figure 4.27. For average goodput, the BSCA algorithm using interference set selection $\gamma_{A}$ achieves higher goodput than TAASCA. Along the same lines of explanation in Section 4.2.3.5. the BSCA algorithm using interference set selection $\gamma_{A}$ fully reuse the twelve available channels for the links in the topology but the TAASCA algorithm partially reuses these channels to separate the global border sets and middle-link set with different channels that sacrifices average goodput. The BSCA algorithm using interference set selection $\gamma_{B}$ achieves less average goodput than the TAASCA algorithm. The BSCA algorithm using interference set selection $\gamma_{C}$ selects fewer channels and does not fully utilise the available channel capacity.

\subsubsection{Discussion about Traffic-aware Anti-starvation Chan- nel Assignment Algorithm}

Simulation validation shows that the TAASCA algorithm effectively resolves flow starvation with different traffic demands and achieves overall best fairness compared with the BSCA CA algorithm using three interference set selections. In both effective and ineffective carrier sensing scenarios, the TUASCA algorithm yields high Jain's index, "zero" starvation link ratio, and low highest-to-lowest goodput ratio with 3 and 12 available channels. Even using 12 available channels, the channel allocation from the BSCA CA algorithm still leads to flow starvation and poor fairness. It further validates the conclusion in Section 4.1 that the interference model used in CA algorithm needs to consider global interference for preventing flow starvation and improving fairness.

However, the TAASCA algorithm has the same weakness as TUASCA that it attains lower average goodput compared with the BSCA CA algo- 
rithm using interference set selection $\gamma_{A}$. The reason is that the TAASCA algorithm partitions the border sets and middle-link set with different channels to prevent flow starvation. But this strategy sacrifices the utilisation of spatial resource and yields lower average goodput. Overall, the TAASCA algorithm achieves better performance in fairness and goodput.

\subsection{Summary}

This chapter aims to answer the second research question: "how can a channel assignment algorithm be designed to prevent flow starvation and improve fairness in IEEE 802.11 WBNs? ".

First, existing interference models used in CA algorithms is the cause of flow starvation and poor network fairness in WBNs. These interference models only reflect the local conflict among adjacent links and lack the consideration of global conflict between border links and middle links. Hence, CA algorithms using these interference models fail to prevent starvation and yield poor fairness.

Addressing the shortcomings of existing interference models, a novel interference model is proposed to account for both global and local interference. Using the new interference model, the newly proposed TUASCA and TAASCA algorithms are designed to prevent flow starvation and improve fairness under saturated and unsaturated traffic assumptions respectively. Simulation results show that the TUAASCA and TAASCA algorithms effectively prevent flow starvation and achieve better fairness compared to the benchmark CA algorithms.

The weakness of TUASCA and TAASCA algorithms is that they focus on improving fairness in ineffective carrier sensing scenarios that sacrifice a part of channel reuse and lead to lower goodput compared to the benchmark CA algorithm. The next chapter will optimise CA algorithms to achieve desired fairness and goodput together. 
128 CHAPTER 4. CHANNEL ASSIGNMENT IMPROVING FAIRNESS 


\section{Chapter 5}

\section{Multi-objective optimisation of channel assignment for improving fairness and goodput in IEEE 802.11 WBNs}

The purpose of the research presented in this chapter is to improve both fairness and goodput via optimised channel assignment (CA) algorithms. This chapter builds on the Chapter 4 which improves fairness by preventing flow starvation.

Fairness and goodput are two essential factors of quality of service (QoS) in IEEE 802.11 WBNs because users not only expect high Internet speed (goodput) but to fairly share the bandwidth (fairness) as well [4. 35. 43]. Hence, fairness and goodput is equally important in IEEE 802.11 WBN planning. However, in high density environments, it is challenging to maximise both fairness and goodput with limited channel resources [26. 83, 84].

To better understand fairness and goodput with limited channel resource, an investigation has been conducted to identify the trade-off between fairness and goodput (see Appendix E). The analysis shows that 
high goodput is an outcome of channel reuse while fairness is governed by the presence of flow starvation. The analysis herein indicates that high goodput and high fairness are a dichotomy with two seemingly opposite goals. Besides the trade-off between goodput and fairness, the analysis in Appendix Ealso shows that different CA algorithms have different focuses on achieving either goodput or fairness. However, existing CA algorithms only target a single objective and do not explicitly target multi-objective optimisation. To achieve both goodput and fairness, the CA should be formulated as a multi-objective optimisation problem.

In a multi-objective optimisation problem, a fitness function is a key element. Such a function is used to measure how close a solution is to the set goal. CA algorithms can iteratively search the solutions that yield desired goodput and fairness based on the evaluation of the fitness function. The fitness function can also be a form of feedback in a closed-loop design [17, 136]. However, existing CA algorithms lack a fitness function to evaluate the output of channel allocation with the set goal and therefore they cannot justify whether the channel output can meet the desired requirement or not before deployment.

Hence, this chapter explains how to evaluate CA algorithms by introducing a new fitness function. Such a function expresses the fairness and goodput from a channel allocation that is representative of the IEEE 802.11 protocol and WBNs. A suitable fitness function provides accurate prediction of performance to evaluate CA algorithms.

With the new fitness function, a multi-objective CA framework is designed to search a set of feasible solutions to meet pre-defined requirements including fairness and goodput. Such a framework allows the integration of different algorithms to utilise their strengths in achieving fairness and goodput. To guarantee the solutions meet the requirements, this framework utilises the fitness function to evaluate the performance of the channel allocation and select the feasible solutions.

One advantage of this multi-objective framework is that the solution 
includes the channel allocation and the prediction of potential goodput, fairness and starvation that have not been explored previously. Another advantage is that the solution set may have multiple feasible solutions instead of one feasible solution, which brings more flexibility to network designers to make the deployment plan. Last but not least, this framework is easy and flexible to integrate new CA algorithms in CA component without influencing the existing components in the framework.

Finally, this framework is applied to develop traffic-unaware and trafficaware MOCA algorithms combined with the fitness function to improve both fairness and goodput for planning IEEE 802.11 WBNs.

The objectives of this chapter are to:

1. Design fitness functions for evaluating the performance of trafficunaware and a traffic-aware CA algorithms.

2. Apply fitness function to design a traffic-unaware multi-objective channel assignment (TUMOCA) to improve both fairness and goodput under the assumption of saturated traffic and validate the TUMOCA algorithm through simulation.

3. Apply fitness function to design a traffic-aware multi-objective channel assignment (TAMOCA) to improve both fairness and goodput under the assumption of unsaturated traffic and validate the TAMOCA algorithm through simulation.

The rest of this chapter is organised as follows. Section 5.1 introduces new QoS fitness functions for evaluating CA algorithms. Sections 5.2 and 5.3 describe the proposed TUMOCA and TAMOCA algorithms followed by the summary in Section 5.4 


\subsection{Fitness Function with Channel Assignment Algorithms}

This section introduces new fitness functions for evaluating the performance of CA algorithms. Such new fitness functions are the fundamental basis of multi-objective optimisation of CA. The next sections first explain the design of the proposed fitness functions and the validation through simulation. A discussion in Section 5.1.4 explains how to utilise the fitness function for evaluating and optimising CA algorithms.

\subsubsection{The Design of Fitness Function}

To evaluate CA algorithms, a fitness function uses the channel allocation from a CA algorithm as an input and calculates the potential performance indicators such as fairness and goodput of the channel allocation before deployment (see Figure 5.1). This fitness function is a cost-effective method for network designers to save time and effort on doing the evaluation through simulations or site survey.

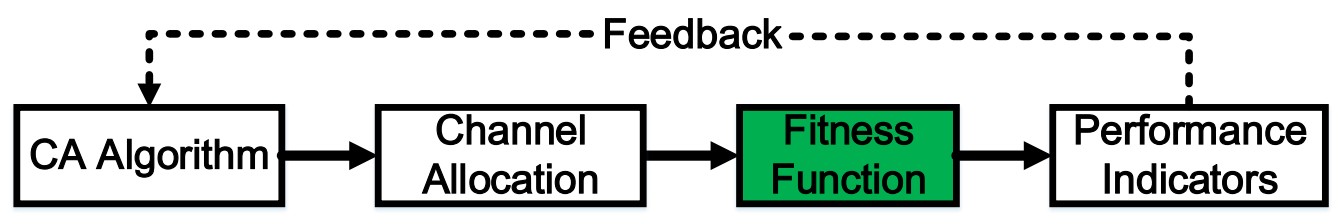

Figure 5.1: evaluating a CA Algorithm with a fitness function

The new fitness function is designed based on the goodput model defined in Chapter 3 and the work in [118]. The similarity between the proposed goodput model and the new designed fitness function is that both of them calculate goodput distribution to evaluate network performance. The difference between them is that the goodput model assumes only one channel is used and the fitness function is flexible for multiple channels. 
The next section starts with a traffic-unaware fitness function with saturated traffic assumption to simplify the calculation of performance and then a traffic-aware fitness function is developed as an extension with consideration of unsaturated traffic demands.

\subsubsection{Traffic-unaware Fitness Function}

The traffic-unaware fitness function does not take traffic demand into account. This function is designed based on the goodput model with saturated traffic assumption in our previous study [116, 118] and Chapter 3 This traffic-unaware fitness function inherits the assumptions from the goodput model with saturated traffic assumption in Section 3.1.1 on page 45 . This traffic-unaware fitness function focuses on capacity contention and ignores capture effect and packet losses caused by collision. All links are assumed to share channels fairly in effective carrier sensing scenarios and border effect exists in ineffective carrier sensing scenarios under saturated traffic assumption.

The inputs of traffic-unaware fitness function are: (i) network link set $E$, (ii) node position $P$, (iii) channel allocation $C A_{\text {output }}$, (iv)available channel set $C$, and $(\mathrm{v})$ the channel sensing range $\left(R_{c S}\right)$ determined by the given physical layer parameters and selected propagation model. The outputs are: (i) goodput distribution for all the links in $E$, (ii) average goodput range $A G_{F E A}$, (iii) Jain's index range $F I_{F E A}$, and (iv) starvation link ratio $S R_{F E A}$.

Definition 29. Normalised average goodput range $A G_{F E A}$

For a given E of an IEEE 802.11 WBN,

$$
A G_{F E A}=\left[\frac{\sum_{i \in E} G_{P}(i)}{N}, \frac{\sum_{i \in E} G_{O}(i)}{N}\right],
$$

where $G_{P}(i)$ and $G_{O}(i)$ are the normalised pessimistic and optimistic goodput predictions of a link $i$ with respect to the maximum net capacity in ineffective carrier sensing scenarios. For effective carrier sensing scenarios, $G_{P}(i)$ and $G_{O}(i)$ are the same, $N$ is the number of links in $E$. 
Definition 30. Jain's index range $F I_{F E A}$

For a given E of an IEEE 802.11 WBN,

$$
F I_{F E A}=\left[\frac{\left(\sum_{i \in E} G_{P}(i)\right)^{2}}{N \times \sum_{i \in E} G_{P}(i)^{2}}, \frac{\left(\sum_{i \in E} G_{O}(i)\right)^{2}}{N \times \sum_{i \in E} G_{O}(i)^{2}}\right],
$$

where $G_{P}(i)$ and $G_{O}(i)$ are the normalised pessimistic and optimistic goodput predictions of a link $i$ with respect to the maximum net capacity in ineffective carrier sensing scenarios, For effective carrier sensing scenarios, $G_{P}(i)$ and $G_{O}(i)$ are the same, $N$ is the number of links in $E$.

Definition 31. Starvation link ratio $S R_{F E A}$

For a given E of an IEEE 802.11 WBN,

$$
S R_{F E A}=\frac{\left|E_{\text {starvation }}\right|}{N}
$$

where $\left|E_{\text {starvation }}\right|$ is the number of links that are predicted to have flow starvation and $N$ is the number of links in $E$. A starvation link is defined as that the achieved goodput of a link is below $\alpha \times G_{\text {average }}$ where $\alpha \in[0.0,0.2]$ is the starvation factor and $G_{\text {average }}$ is the average goodput in $E$.

The logic flow and the pseudo code of the traffic-unaware fitness function are shown in Figure 5.2 and Algorithm 8 . First, the fitness function groups the links according to the allocated channel (see line 3). Second, the fitness function calculates the goodput distribution in each group separately. For the links in each group using an identical channel, the fitness function calculates the border distance $D$ (see lines 4 ).

Third, comparing border distance $D$ with the carrier sensing range $R_{c s}$, the fitness function checks whether the topology of the current channel group belongs to effective or ineffective carrier sensing scenarios (see line 5). For an effective carrier sensing scenario, the fitness function will calculate goodput distribution using the goodput model defined in Eq. 3.1 in Section 3.1.1.1 on page 46 (see line 5). For an ineffective carrier sensing scenario, the fitness function will calculate goodput distribution using 


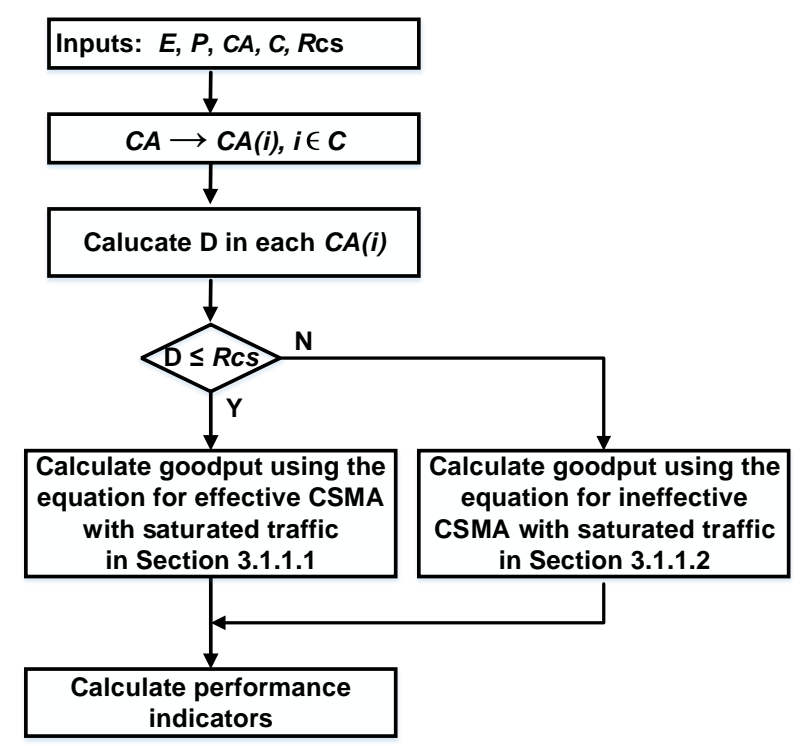

Figure 5.2: The logic flow of traffic-unaware fitness function

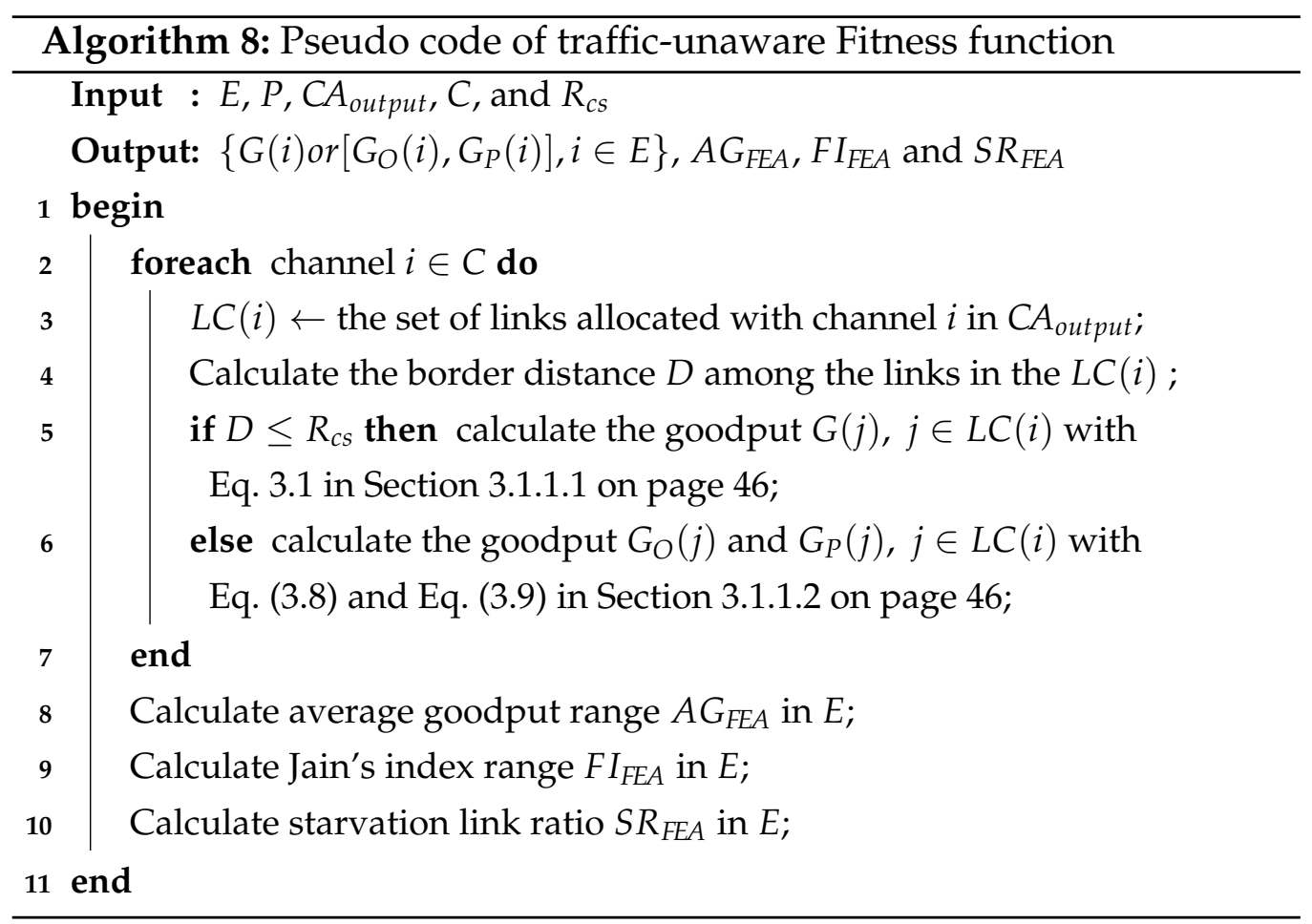


the goodput model defined in Eq. (3.8) and Eq. (3.9) in Section 3.1.1.2 on page 46 (see line 6). In the end, the fitness function calculates the performance indicators $A G_{F E A}, F I_{F E A}$ and $S R_{F E A}$ based on the goodput distribution information (see lines 8-10).

\subsubsection{Traffic-aware Fitness Function}

Different from the above traffic-unaware fitness function, the traffic-aware fitness function will consider traffic demand to calculate the performance indicators. The traffic-aware fitness function is designed based on the goodput model with unsaturated traffic assumptions that the occurrence of the border effect depends on the traffic demand from the border link sets (see Section 3.1 .2 on page 53).

The inputs of traffic-aware fitness function are: (i) network link set $E$, (ii) node position $P$, (iii) channel allocation $C A_{\text {output }}$ (iv) traffic demand $f$, and (v) available channel set $C$, and (vi) fixed $R_{c s}$. The outputs are: (i) goodput distribution for all links in $E$, (ii) average goodput range $A G_{F E A}$, and (iii) fairness index range $F I_{F E A}$, and (iv) starvation link ratio $S R_{F E A}$.

The logic flow and the pseudo code of the traffic-aware fitness function are shown in the Figure 5.3 and Algorithm 9. The main difference between the traffic-aware fitness function and traffic-unaware fitness function is that the traffic-aware fitness function calculates goodput distribution with consideration of traffic demand in both effective and ineffective carrier sensing scenarios.

First, the fitness function groups the links according to the allocated channel (see line 3). Second, the fitness function calculates the goodput distribution in each group separately. For the links in each group using an identical channel, the fitness function calculates the border distance $D$ (see lines 4). Third, comparing border distance $D$ with the carrier sensing range $R_{c s}$, the fitness function checks whether the topology of the current channel group belongs to effective or ineffective carrier sensing scenarios (see line 5). 


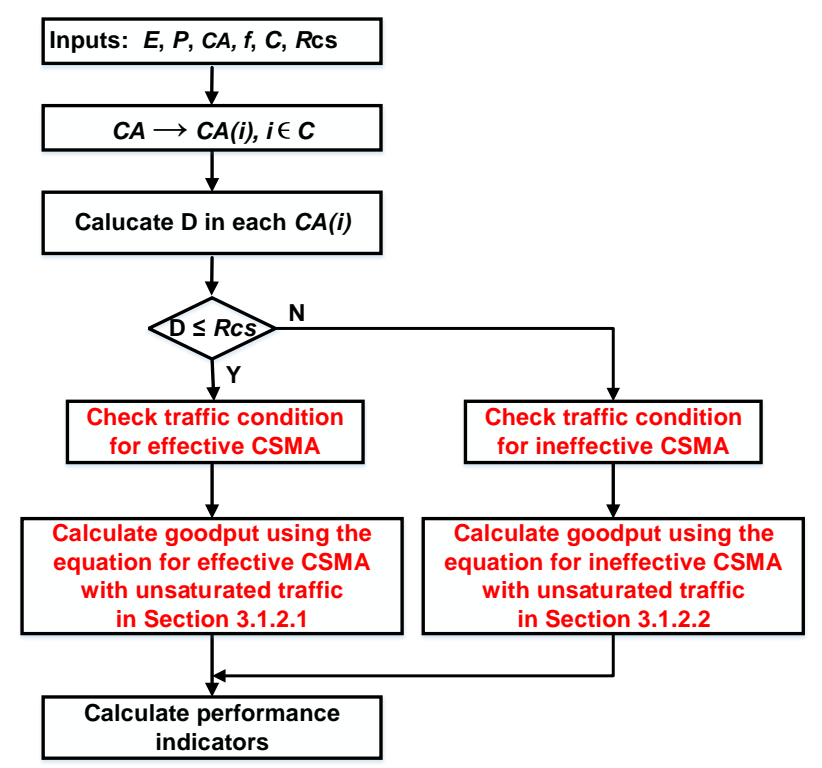

Figure 5.3: The logic flow of traffic-aware fitness function

For an effective carrier sensing scenario, the traffic-aware fitness function will check the traffic condition $\left(\sum_{j \in L C(i)} f(j)>1\right.$ where $L C(i)$ is a group of links using an identical channel $i$ and $f(j)$ is the traffic demand). This condition checks whether the channel capacity is sufficient for the traffic demands among those links using channel $i$. Then the fitness function calculates goodput $G(j)$ of a link $j$ by using corresponding Eq. 3.10 with the outcome of the traffic condition in Section 3.1.2.1 on page 54 (see line 5).

For an ineffective carrier sensing scenario, two criteria, A and B, are defined to refine the possible combinations of unsaturated traffic demands. First, criterion A is to evaluate whether border effect occurs. It is defined as the sum of traffic demands of the links in the dominant border-link sets $G I S_{L B}$ and $G I S_{R B}$, and expressed as: $\sum_{j \in G I S_{L B} \cap G I S_{R B}} f(j) \geq 1$. Criterion $B$ is the sum of traffic demands of the links in the global left or right border links with the links in its conflict set $\gamma(B)$, and this is expressed as: $\sum_{j \in B \cap \gamma(B)} f(j) \geq 1$. The criterion B is to evaluate whether the channel ca- 


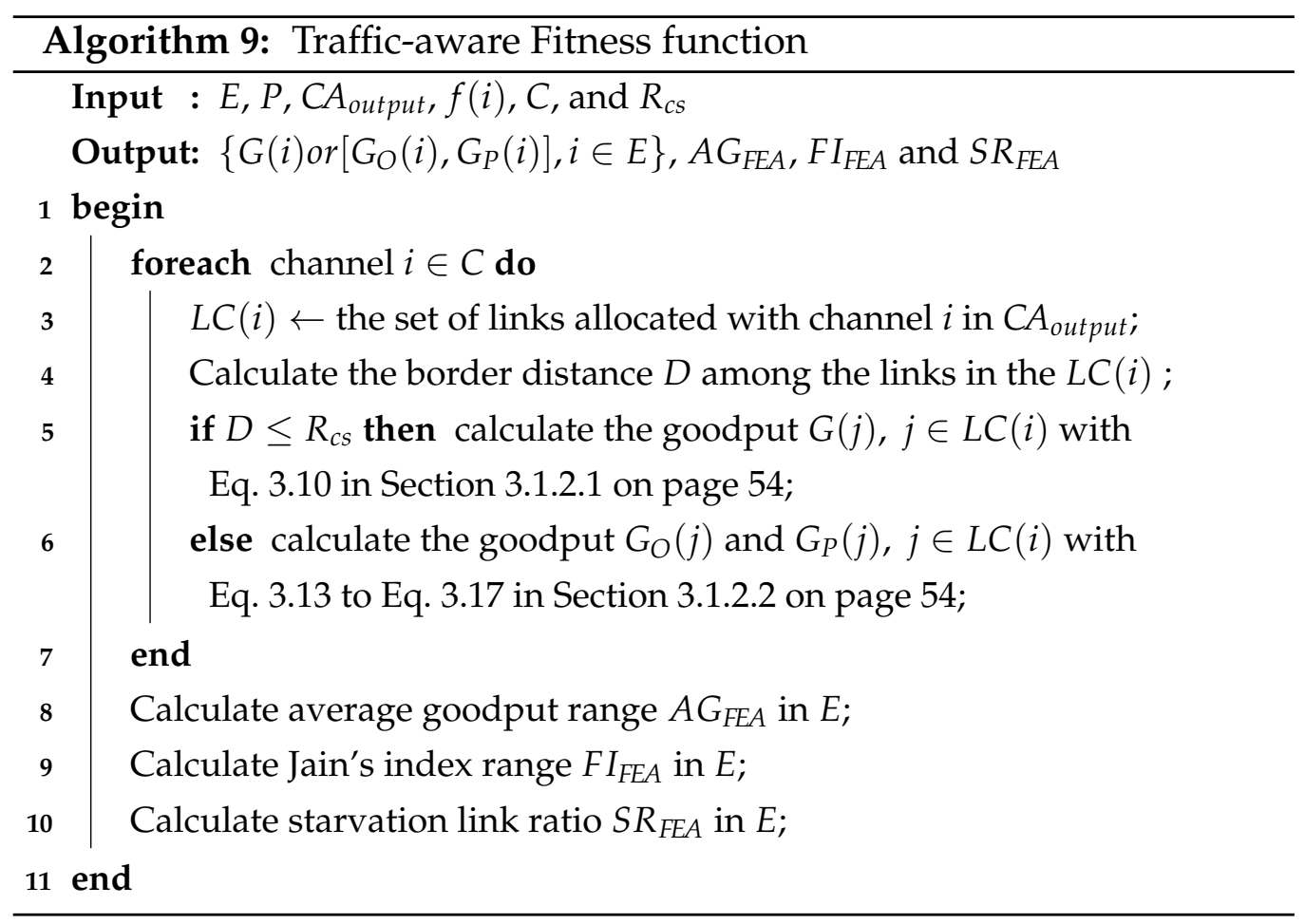

pacity is sufficient for the border link and the links in its conflict set. More details can be found in Section 3.1.2.2 on page 54 .

According to the above two criteria, the fitness function calculates the goodput distribution with Eq. 3.13 to Eq. 3.17 in Table 3.3 in Section 3.1.2.2 (see line 6).

In the end, the fitness function calculates the performance indicators $A G_{F E A}, F I_{F E A}$ and $S R_{F E A}$ based on the goodput distribution information (see lines 8-10).

\subsubsection{Validation of Traffic-unaware Fitness Function}

This subsection validates the proposed traffic-unaware fitness function through simulation from two aspects. First, we validate the accuracy of the evaluation of the traffic-unaware fitness function in the scenarios using different topologies compared with simulation results. Second, we 
Table 5.1: Simulation Parameters

\begin{tabular}{l|l}
\hline Parameter Name & Value \\
\hline Transmission Power & $18 \mathrm{dBm}$ \\
\hline Receiver Sensitivity & $-78 \mathrm{dBm}$ \\
\hline Path Loss Model & Two-Ray \\
\hline Shadowing \& Fading Model & None \\
\hline MAC Layer & PCS \\
\hline Routing & Static Routing \\
\hline Transportation Layer & UDP \\
\hline Physical Layer & IEEE802.11a \\
\hline Data Rate & $24 \mathrm{Mbps}$ \\
\hline Packet Size & $1500 \mathrm{Bytes}$ \\
\hline Interpacket Interval & $0.5 \mathrm{~ms}$ \\
\hline
\end{tabular}

compare the traffic-unaware fitness function with two evaluation metrics TID [81, 102, 140] and CDAL cost [82] through all simulations. These two evaluation metrics have been commonly used to evaluate the performance of CA algorithms [81].

The channel allocations used in the validation are from a standard clique-based CA algorithm INSTC [140] (see Section 4.2.2 on page 92).

The validation is conducted in QualNet 5.2 simulator. The corresponding values for the input parameters are given in Table 5.1. With the simulation configuration, $R_{c s}$ is calculated as $515 \mathrm{~m}$ and $D_{t r}^{\max }$ is calculated as $207 \mathrm{~m}$. The interference range $R_{I}$ is defined as $2 \times D_{t r}^{\max }\left(D_{t r}^{\max }\right.$ is $\left.207 \mathrm{~m}\right)$. The channel indices $C$ as $\{1,2,3\}$. All links are configured as unicast constant-bit rate transmission with saturated traffic demand (24 Mbps).

All averages of simulation results shown are reported with confidence interval of $95 \%$ with the range from $[2.716,34.359]$ kbps under the assumption that the averages are normally distributed. 


\subsubsection{Different Network Sizes}

The grid topologies used in this subsection range from $D=200 \mathrm{~m}$ to $800 \mathrm{~m}$ with a constant interval $d$ of $50 \mathrm{~m}$ (Fig. 5.4).

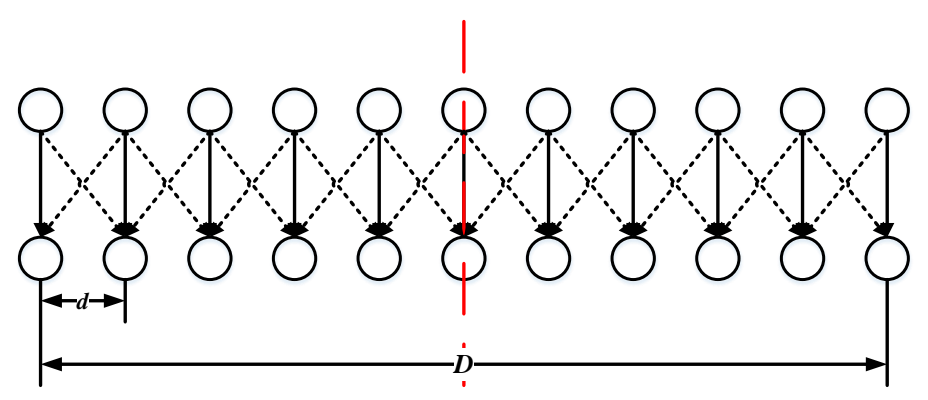

Figure 5.4: A topology representing a point-to-point IEEE 802.11 WBN

In Tables 5.2 and 5.3 , performance indicators calculated by the trafficunaware fitness function are compared with simulation results. Performance indicators include $A G_{F E A}, S R_{F E A}$, and $F I_{F E A}$ while $A G_{S}, S R_{S}$, and $F I_{S}$ represent corresponding simulation results (see definitions in 4.2.3.1 on page 97). We mark the cases that the simulation result does not match with the prediction from the fitness function with *. The error ratio is calculated based on Def. 16 on page 61 .

The proposed fitness function performs well in the grid topologies with accurate prediction of average goodput, and starvation ratio. All simulation results of average goodput and starvation link ratio match with the prediction from the fitness function. In terms of Jain's index, in ineffective carrier sensing scenarios $(600 \mathrm{~m}$ and $800 \mathrm{~m})$, the simulation results are slightly higher than the prediction from the fitness function and the overall error of the prediction is below $7.5 \%$. The reason for the error in the $600 \mathrm{~m}$ case is that carrier sensing range is not a binary function and the irregularity of carrier sensing exists. However, in the proposed goodput model, the carrier sensing range is fixed without considering the irregularity. 
Table 5.2: Validation of average goodput in grid topologies with the INSTC CA

\begin{tabular}{ccccc}
\hline Network Size & $A G_{s}$ & $A G_{\text {FEA }}$ & $T I D$ & $C D A L_{\text {cost }}$ \\
\hline $200 \mathrm{~m}$ & 0.5998 & 0.600 & 5 & 0.577 \\
$400 \mathrm{~m}$ & 0.333 & 0.333 & 9 & 0.000 \\
$600 \mathrm{~m}$ & 0.291 & {$[0.285,0.308]$} & 25 & 0.577 \\
$800 \mathrm{~m}$ & 0.335 & {$[0.322,0.353]$} & 66 & 0.577 \\
\hline
\end{tabular}

Table 5.3: Validation of fairness in grid topologies with the INSTC CA

\begin{tabular}{ccccccc}
\hline Network Size & $S R_{S}$ & $S R_{\text {FEA }}$ & $F I_{S}$ & $F I_{\text {FEA }}$ & $T I D$ & $C D A L_{\text {cost }}$ \\
\hline $200 \mathrm{~m}$ & 0.000 & 0.000 & 0.900 & 0.900 & 5 & 0.577 \\
$400 \mathrm{~m}$ & 0.000 & 0.000 & 1.000 & 1.0000 & 9 & 0.000 \\
$600 \mathrm{~m}$ & 0.231 & 0.231 & $0.548^{*}$ & {$[0.492,0.508]$} & 25 & 0.577 \\
$800 \mathrm{~m}$ & 0.412 & 0.412 & $0.539^{*}$ & {$[0.482,0.502]$} & 66 & 0.577 \\
\hline
\end{tabular}

\subsubsection{Different Evaluation Metrics}

This subsection compares the proposed fitness function with another two CA evaluation metrics, $T I D$ and $C D A L_{\text {cost }}$. The calculation of TID metric is defined based on Algorithm 10 according to the definition in [81]. The metric TID calculates the total sum of the number of interfering links for all links in an IEEE 802.11 WBN. In this thesis, the interfering link is defined based on the $R_{I}$ (see Def. 32). The $C D A L_{\text {cost }}$ metric was proposed in [82] as part of a larger algorithm for channel distribution across links. The last part of Algorithm 1 in [82] is used to calculate the $C D A L_{\text {cost }}$ metric. The metric $C D A L_{\text {cost }}$ calculates the number of links allocated to the same channel and then derives the standard deviation among the available channels.

Definition 32. Interfering set $\gamma_{B}$

Let $E$ denote the complete set of links in an IEEE 802.11 WBN. For a tagged link 
$i$ in $E$, the conflict set,

$$
\gamma_{B}(i)=\left\{l \in E \backslash\{i\} \mid d_{s, r} \leq R_{I}\right\},
$$

whereby $d_{s, r}$ is the distance between sender and receiver in link $l$ and link $i$ respectively, is the theoretical interference range $\left(R_{I}=k \times D_{t r}^{\max }, k=2\right)$.

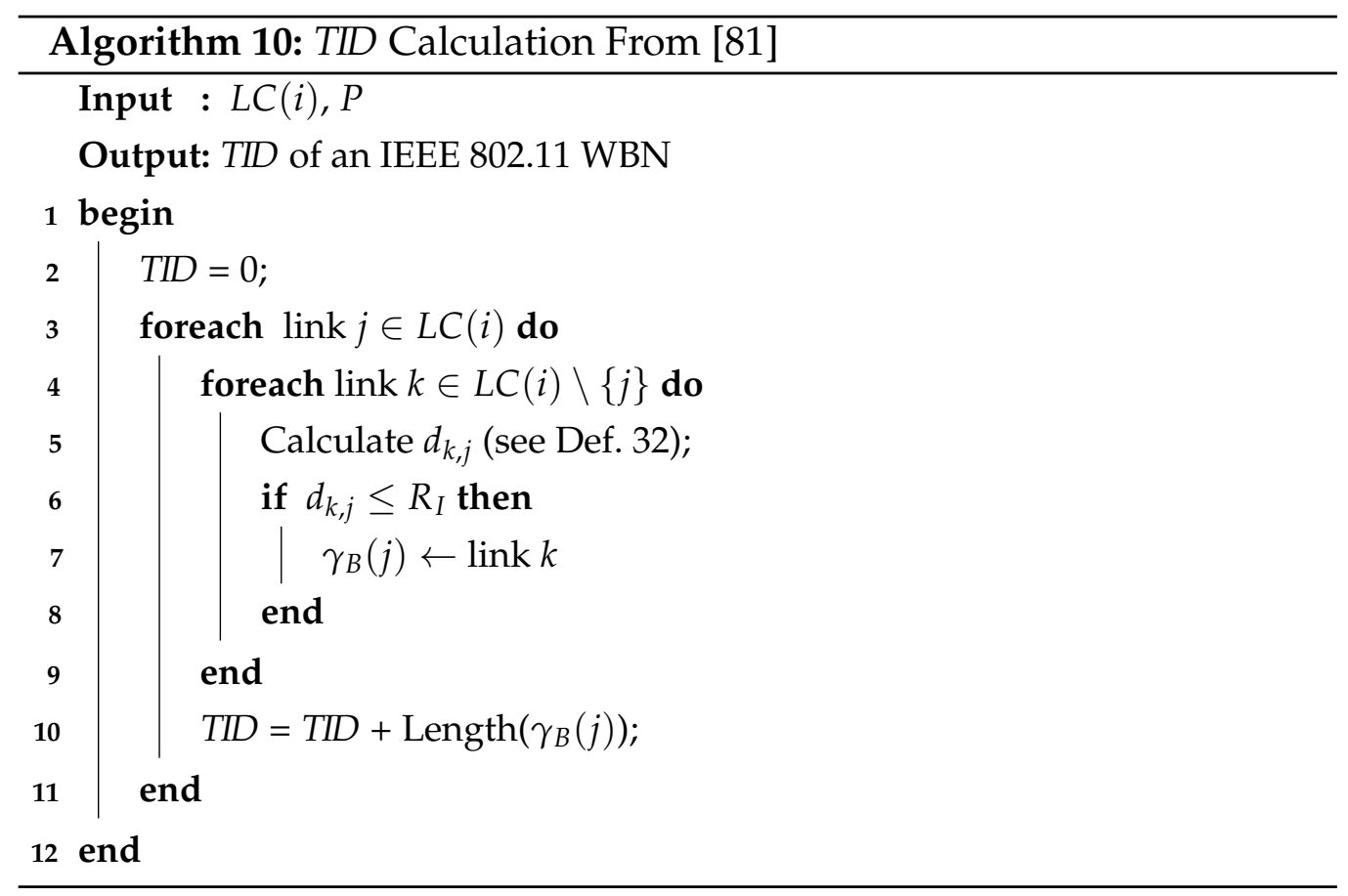

The metrics TID and CDAL $L_{\text {cost }}$ compare with goodput and fairness indicators for all above scenarios in Tables 5.2 and 5.3. Both TID and CDAL $L_{\text {cost }}$ cannot reflect the trends of average goodput, starvation link ratio, and fairness index with different network sizes. For example, in the $400 \mathrm{~m}$ and $800 \mathrm{~m}$ scenarios, goodput values from simulations are similar but TID and $C D A L_{\text {cost }}$ have different values. In the $200 \mathrm{~m}$ and $600 \mathrm{~m}$ scenarios, $C D A L_{\text {cost }}$ values are the same but the goodput, starvation link ratio, and Jain's index are much different. These two metrics are not sensitive to the trend of average goodput, starvation link ratio, and Jain's index. Hence, these two metrics are not suitable for evaluating CA algorithms. 
Overall, the proposed traffic-unaware fitness function provides accurate prediction of the performance of channel allocation and outperforms the other two evaluation metrics.

\subsubsection{Validation of Traffic-aware Fitness Function}

This subsection validates the proposed traffic-aware fitness function through simulation from the same two aspects as those in the above Section. The simulation tool, configuration, topologies, and benchmark performance metrics are defined as same as those in Section 5.1.2. A balanced static CA (BSCA) algorithm [87] is selected to generate channel allocations for simulation (see Algorithm 7 in Section 4.3.2 on page 114) to generate the channel allocations. Five normalised traffic demands with respect to the maximum net bandwidth are selected as 1.0, 0.8, 0.6, 0.4, and 0.1 representing different level of traffic conditions. All the traffic demands are calculated at application layer. All averages of simulation results shown are reported with confidence interval of $95 \%$ with the range from [2.746,34.359] $\mathrm{kbps}$ under the assumption that the averages are normally distributed.

\subsubsection{Different Network Sizes}

Table 5.4: Validation of average goodput in grid topology $D=400 \mathrm{~m}$ with the BSCA CA

\begin{tabular}{ccccc}
\hline Traffic Demand & $A G_{s}$ & $A G_{F E A}$ & $T I D$ & $C D A L_{\text {cost }}$ \\
\hline 1.0 & 0.333 & 0.333 & 9 & 0.000 \\
0.8 & 0.333 & 0.333 & 9 & 0.000 \\
0.6 & 0.333 & 0.333 & 9 & 0.000 \\
0.4 & 0.333 & 0.333 & 9 & 0.000 \\
0.2 & 0.200 & 0.200 & 9 & 0.000 \\
\hline
\end{tabular}


Table 5.5: Validation of fairness in grid topology $D=400 \mathrm{~m}$ with the BSCA CA

\begin{tabular}{ccccccc}
\hline Traffic Demand & $S R_{S}$ & $S R_{\text {FEA }}$ & $F I_{S}$ & $F I_{\text {FEA }}$ & $T I D$ & $C D A L_{\text {cost }}$ \\
\hline 1.0 & 0.000 & 0.000 & 0.996 & 0.999 & 9 & 0.000 \\
0.8 & 0.000 & 0.000 & 0.996 & 0.999 & 9 & 0.000 \\
0.6 & 0.000 & 0.000 & 0.996 & 0.999 & 9 & 0.000 \\
0.4 & 0.000 & 0.000 & 0.996 & 0.999 & 9 & 0.000 \\
0.2 & 0.000 & 0.000 & 0.999 & 0.999 & 9 & 0.000 \\
\hline
\end{tabular}

Here, two network sizes are used as examples to show the validation of the traffic-aware fitness function. One network size is $400 \mathrm{~m}$ belonging to the effective carrier sensing scenario and another one is $800 \mathrm{~m}$ belonging to the ineffective carrier sensing scenario.

Tables 5.4 to 5.7 show that the evaluation from the traffic-aware fitness function is very close to the simulation results. It proves that with different traffic demands, the traffic-aware fitness function can provide accurate performance evaluation of CA algorithms.

Table 5.6: Validation of average goodput in grid topology $D=800 \mathrm{~m}$ with the BSCA CA

\begin{tabular}{ccccc}
\hline Traffic Demand & $A G_{s}$ & $A G_{\text {FEA }}$ & $T I D$ & $C D A L_{\text {cost }}$ \\
\hline 1.0 & 0.333 & {$[0.324,0.353]$} & 66 & 0.577 \\
0.8 & 0.333 & {$[0.324,0.353]$} & 66 & 0.577 \\
0.6 & 0.330 & {$[0.258,0.330]$} & 66 & 0.577 \\
0.4 & 0.291 & {$[0.254,0.318]$} & 66 & 0.577 \\
0.2 & 0.200 & {$[0.200,0.200]$} & 66 & 0.577 \\
\hline
\end{tabular}


Table 5.7: Validation of fairness in Grid Topology $D=800 \mathrm{~m}$ with the BSCA CA

\begin{tabular}{ccccccc}
\hline Traffic Demand & $S R_{S}$ & $S R_{\text {FEA }}$ & $F I_{S}$ & $F I_{\text {FEA }}$ & $T I D$ & $C D A L_{\text {cost }}$ \\
\hline 1.0 & 0.294 & 0.294 & 0.665 & {$[0.585,0.635]$} & 66 & 0.577 \\
0.8 & 0.294 & 0.294 & 0.665 & {$[0.585,0.635]$} & 66 & 0.577 \\
0.6 & 0.294 & 0.294 & 0.681 & {$[0.642,0.653]$} & 66 & 0.577 \\
0.4 & 0.000 & 0.000 & 0.861 & {$[0.858,0.858]$} & 66 & 0.577 \\
0.2 & 0.000 & 0.000 & 0.999 & {$[0.999,1.000]$} & 66 & 0.577 \\
\hline
\end{tabular}

\subsubsection{Different Evaluation Metrics}

In Tables 5.4 to 5.7, two metrics TID and $C D A L_{\text {cost }}$ keep the constant value for the channel allocation with different traffic demands. It implies that $T I D$ and $C D A L_{\text {cost }}$ are not sensitive to the change of traffic demand. The proposed traffic-aware fitness function outperforms TID and CDAL $L_{\text {cost }}$ in predicting three performance indicators.

\subsubsection{Summary of Fitness Function}

This section introduces two fitness functions to evaluate the performance of CA algorithms under saturated and unsaturated traffic conditions. Simulation results show that two fitness functions provide accurate prediction of performance indicators for evaluating CA algorithms. Moreover, the new fitness functions are compared with other two evaluation metrics in terms of average goodput, starvation link ratio, and fairness index. The proposed fitness functions outperforms these two evaluation metrics for all performance indicators.

Besides accuracy, simplicity is another strength of the proposed fitness functions. The computational complexity of the proposed fitness function is $\mathrm{O}(\mathrm{N})$, where $\mathrm{N}$ is the number of links in the given IEEE 802.11 WBN. With low computational complexity, the calculation of the proposed fit- 
ness function will be efficient.

With advantages of simplicity and accuracy, these new fitness functions are useful to evaluate $\mathrm{CA}$ algorithms in the planning stage. Besides using the fitness function to evaluate $\mathrm{CA}$ algorithms, the performance indicators from the fitness function can be fed back to $C A$ algorithms and $C A$ algorithms can adjust the computation with this feedback (see Figure 5.1).

The next section will use the new fitness functions to design multiobjective CA algorithms to improve fairness and goodput in IEEE 802.11 WBNs.

\subsection{A Traffic-unaware Multi-objective Channel As- signment Algorithm For Improving Fairness and goodput}

This section aims to develop a traffic-unaware multi-objective CA (TUMOCA) algorithm to improve both fairness and goodput. Different from other CA algorithms discussed in Chapter 2, the proposed TUMOCA algorithm aims to address both fairness and goodput. This TUMOCA algorithm takes pre-defined requirements into account and aims to find a set of feasible solutions of channel allocation to meet the predefined requirements for a given IEEE 802.11 WBN. The predefined QoS requirement includes starvation link ratio and Jain's index as fairness requirements, and average goodput as goodput requirements.

The next subsections introduce the design of the TUMOCA algorithm and validate it through simulation. 


\subsubsection{The Design of Traffic-unaware Multi-objective Chan- nel Assignment Algorithm}

The reason for ignoring the traffic pattern is to simplify the problem, and also that the traffic pattern is unknown at the planning stage. Therefore, saturated traffic assumption is used representing the heaviest traffic condition in this section.

The discussion in Section 5.1.4 stated that developing a CA algorithm with the evaluation from a fitness function can help improve the performance of CA algorithms. Hence, the new TUMOCA algorithm is designed based on a joint framework (see Figures 5.1 and 5.5).

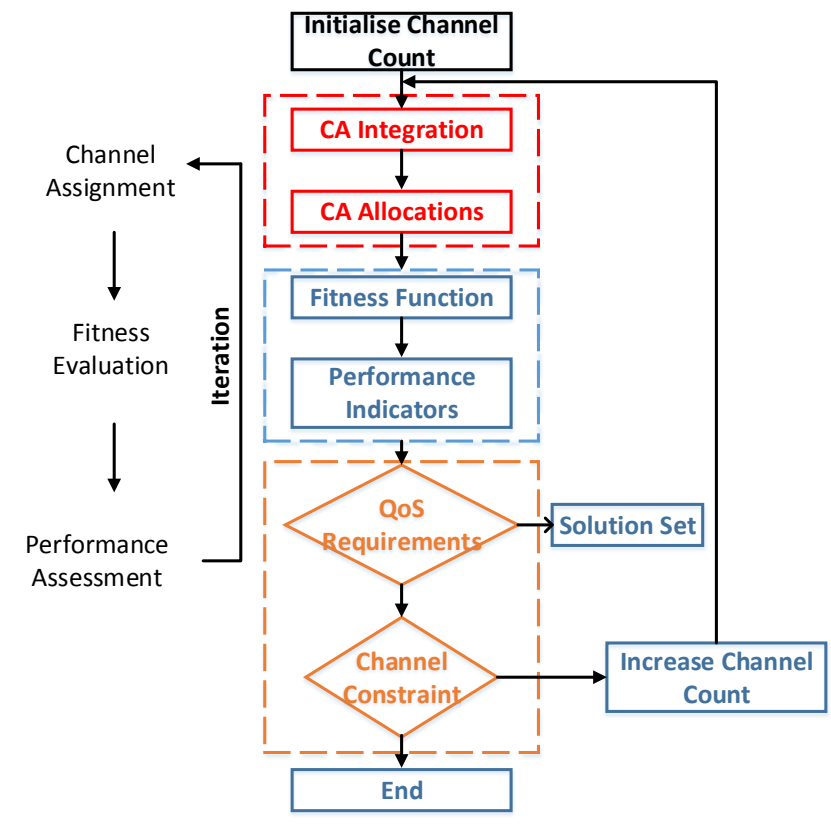

Figure 5.5: The joint framework

The new joint framework has three main components: CA component, fitness evaluation component, and performance assessment component. The CA component embeds different CA algorithms to utilise 
different strengths. The fitness evaluation component uses fitness function to calculate the performance indicators of the channel allocation generated from the CA component. The performance assessment component assesses whether the potential performance of a channel allocation meets pre-defined performance requirements. If the channel allocation does meet the requirements, the allocation is put into a solution set. If not, the system will iterate increasing the channel count until the channel count reaches the maximum number of available channels. In the worse case, the TUMOCA yields an empty solution set without any feasible solution. In this case, to find feasible solutions, the user of TUMOCA algorithm needs either to relax the pre-defined performance requirement or increase the number of available channels.

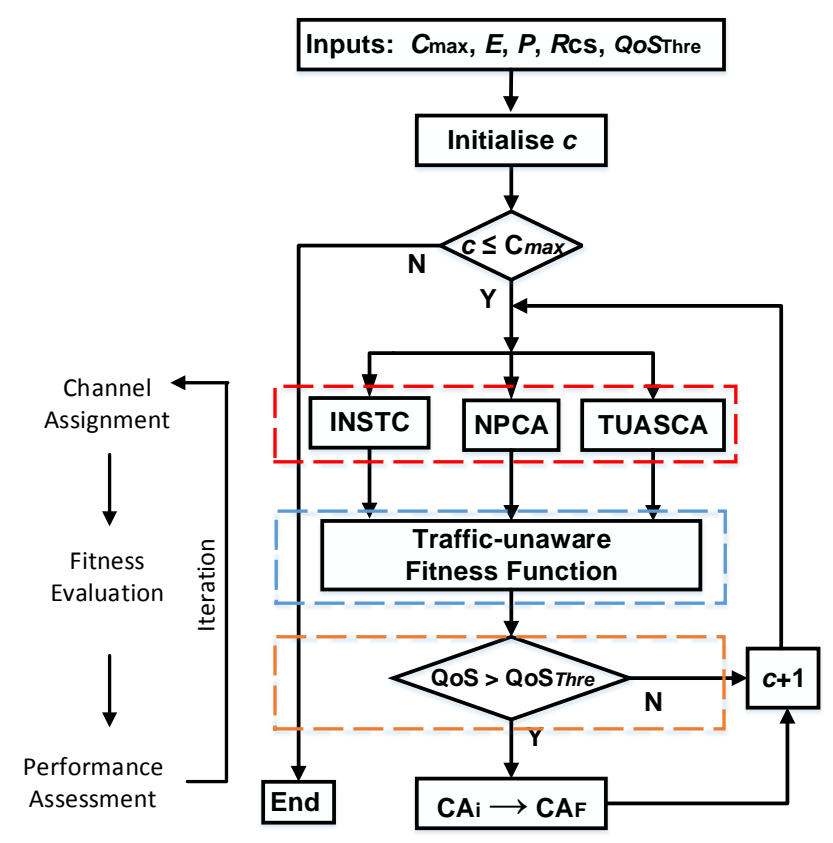

Figure 5.6: The logic flow of TUMOCA algorithm

Next, this joint design structure is applied to the TUMOCA algorithm. In the TUMOCA (see Figure 5.6), the CA component embeds three CA al- 
gorithms, INSTC [140] (see Section 4.2.2.4 on page 95), NPCA [130] (see Section 4.2.1 on page 84, and TUASCA (see Section 4.2.1 on page 84). Simulation analyses show that these three CA algorithms have different strengths in achieving higher goodput, better fairness or preventing flow starvation (see Appendix E.4). In effective carrier sensing scenarios, all three CA algorithms achieve identical goodput and fairness because carrier sensing mechanism manages the medium access without exposed nodes, hidden nodes, and border effect. However, in ineffective carrier sensing scenarios, INSTC algorithm achieves the highest goodput but poor fairness. NPCA algorithm achieves highest fairness but poor goodput while TUASCA algorithm maintains high fairness and medium level of goodput between INSTC and NPCA.

The fitness function in the TUMOCA algorithm is using the trafficunaware fitness function defined in Section 5.1. The inputs of TUMOCA algorithm are link set $E$, node position $P$, maximum channel number $C_{\max }$, and predefined QoS threshold $Q o S_{\text {Thre }}$ including $A G_{\text {Thre }}, F I_{\text {Thre }}$, and $S R_{\text {Thre }}$. The output of TUMOCA algorithm is a set of channel allocations that meet the $Q o S_{\text {Thre }}$ and the performance indicators of each channel allocation.

The pseudo code of the TUMOCA algorithm is shown in Algorithm 11 At the beginning, TUMOCA initialises channel number $c$ (see line 2) and then starts the iteration of $c$. For each iteration, TUMOCA has two steps. First, TUMOCA algorithm runs three $C A$ algorithms with the current value of $c$ and yields three channel allocations that form a set of temporary solution $C A_{\text {temp }}$ in lines $4-7$. In the second step, TUMOCA runs the trafficunaware fitness function to calculate the performance indicators $Q O S_{F E A}$ line 9. In lines $10-12$, TUMOCA algorithm compares the lower bound of $Q O S_{F E A}$ with the pre-defined QoS threshold QoS $S_{\text {Thre }}$. If the $Q O S_{F E A}$ is greater than $Q o S_{\text {Thre }}$, TUMOCA accepts the channel allocation as a feasible solution. If not, TUMOCA algorithm increases $c$ by 1 in line 13 and goes to the next iteration. The TUMOCA stops and outputs the solution set $C A_{F}$ when $c$ is greater than the maximum channel number $C_{\max }$. 


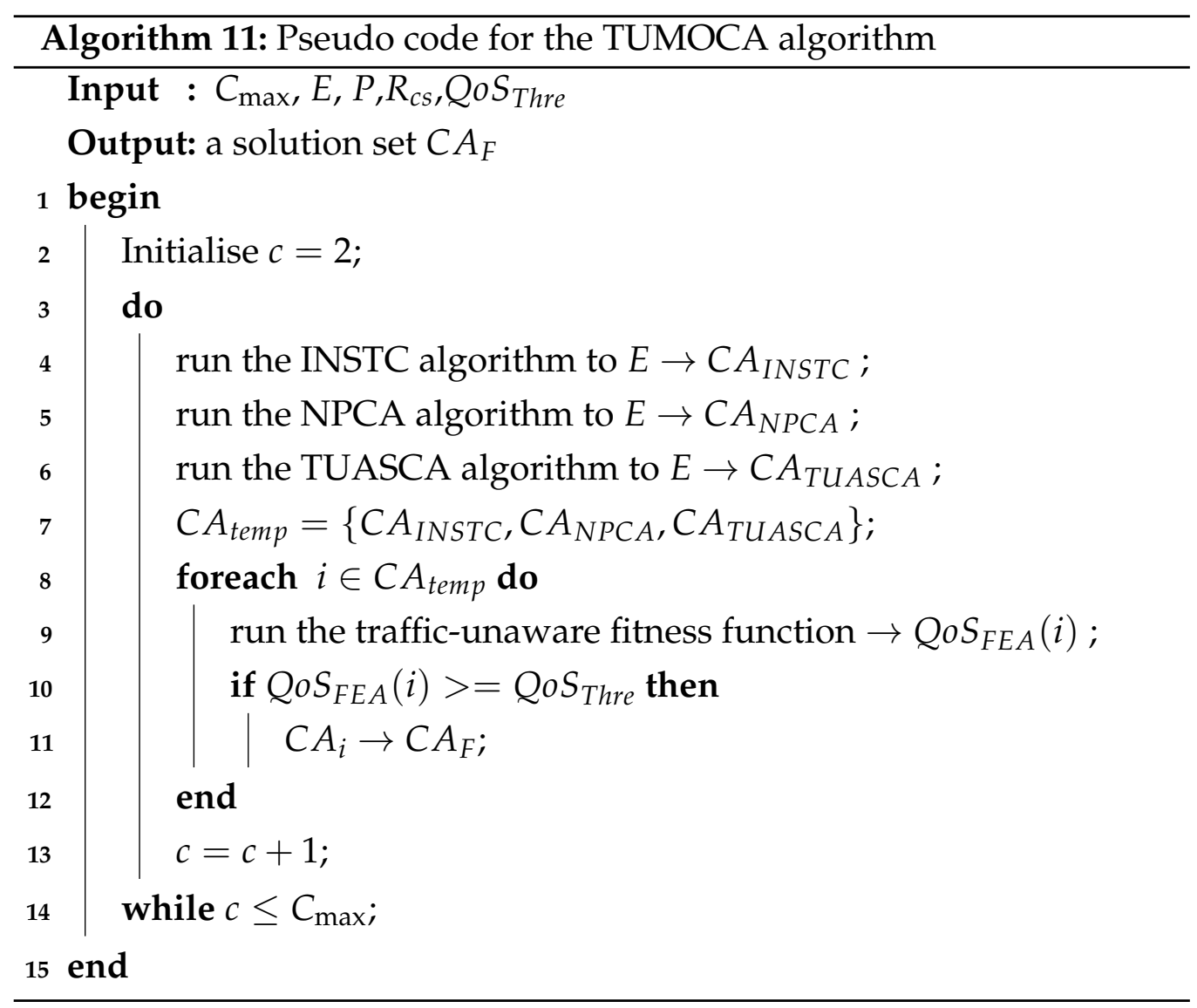

\subsubsection{Validation of Traffic-unaware Multi-objective Chan- nel Assignment Algorithm}

This subsection validates the TUMOCA algorithm through simulation. As goodput and fairness are satisfactory in effective carrier sensing scenarios (more details see Appendix E), the examples are listed in this section mainly to show the validation in ineffective carrier sensing scenarios. The topology used as an example is a $D=800 \mathrm{~m}, d=50 \mathrm{~m}$ and $D_{t r}=50 \mathrm{~m}$ in Figure 5.4. The maximum channel number is selected as $3,4,6$.

The simulation runs with Qualnet 5.2 by using IEEE 802.11 a parameters in Table 5.8. All links are configured to unicast transmission with constant bit rate carrying maximum traffic (i.e saturated link assumption). 
With the above configuration, the TUMOCA algorithm is validated by using different channel numbers and different performance requirements. The method of validation is to calculate the performance of the channel allocations from the TUMOCA in simulations. If the simulation results meet the performance requirement, the TUMOCA algorithm meets the expectation. In addition, the TUMOCA algorithm will be compared with three $\mathrm{CA}$ algorithms in Appendix E to show the difference between them.

All averages of simulation results shown are reported with confidence interval of $95 \%$ with the range from $[3.6,16.962]$ kbps under the assumption that the averages are normally distributed.

Table 5.8: Simulation Parameters for validating TUMOCA

\begin{tabular}{l|l}
\hline Parameter Name & Value \\
\hline Transmission Power & $18 \mathrm{dBm}$ \\
\hline Receiver Sensitivity & $-78 \mathrm{dBm}$ \\
\hline Path Loss Model & Two-Ray \\
\hline Shadowing \& Fading Model & None \\
\hline MAC Layer & PCS \\
\hline Routing & Static Routing \\
\hline Transportation Layer & UDP \\
\hline Physical Layer & IEEE802.11a \\
\hline Data Rate & $24 \mathrm{Mbps}$ \\
\hline Packet Size & $1500 \mathrm{Bytes}$ \\
\hline Interpacket Interval & $0.5 \mathrm{~ms}$ \\
\hline Transmission Range & $50 \mathrm{~m}$ \\
\hline
\end{tabular}

First, the predefined QoS requirement is set as $S R_{\text {Thre }}=0.0, F I_{\text {Thre }}=$ 0.7 , and $A G_{\text {Thre }}=0.2$. The results from the TUMOCA algorithm using 3 and 4 channels are listed in Tables 5.9 to 5.12. These four tables list the solution set from the TUMOCA algorithm with goodput and fairness requirements respectively. The $C h_{\text {need }}$ column represents the channel count 
needed in the corresponding allocation. The "Validation" column shows whether the solution in the row meets the corresponding requirement.

Table 5.9: The solution set for a $D=800 \mathrm{~m}$ grid topology with three available channels and goodput requirement

\begin{tabular}{c|c|cccc}
\hline Index & $C h_{\text {need }}$ & $A G_{\text {FEA }}$ & $A G_{S}$ & $A G_{\text {Thre }}$ & Validation \\
\hline TUMOCA\#1 & 3 & {$[0.229,0.235]$} & 0.223 & 0.2 & $\checkmark$ \\
\hline
\end{tabular}

Table 5.10: The solution set for a $D=800 \mathrm{~m}$ grid topology with three available channels and fairness requirements

\begin{tabular}{c|c|cccc|cccc}
\hline Index & $C h_{\text {need }}$ & $S R_{F E A}$ & $S R_{S}$ & $S R_{\text {Thre }}$ & Validation & $F I_{\text {FEA }}$ & $F I_{S}$ & $F I_{\text {Thre }}$ & Validation \\
\hline TUMOCA\#1 & 3 & 0.000 & 0.000 & 0.0 & $\checkmark$ & {$[0.897,0.911]$} & 0.895 & 0.7 & $\checkmark$ \\
\hline
\end{tabular}

Table 5.11: The solution set for a $D=800 \mathrm{~m}$ grid topology with four available channels and goodput requirements

\begin{tabular}{c|c|cccc}
\hline Index & $C h_{\text {need }}$ & $A G_{\text {FEA }}$ & $A G_{s}$ & $A G_{\text {Thre }}$ & Validation \\
\hline TUMOCA\#1 & 3 & {$[0.229,0.235]$} & 0.223 & 0.2 & $\checkmark$ \\
TUMOCA\#2 & 4 & {$[0.224,0.235]$} & 0.218 & 0.2 & $\checkmark$ \\
TUMOCA\#3 & 4 & {$[0.285,0.294]$} & 0.276 & 0.2 & $\checkmark$ \\
\hline
\end{tabular}

For this scenario $(D=800 \mathrm{~m})$ with three channels, the TUMOCA algorithm yields one solution that the channel count needed is 3 . When the count of available channels is increased from three to four with the same QoS requirement and this time TUMOCA yields three channel allocations in Tables 5.11 and 5.12 .

Simulation results show that all the solutions from the TUMOCA algorithm meet the pre-defined QoS requirements. When four channels are 
Table 5.12: The solution set for a $D=800 \mathrm{~m}$ grid topology with four available channels and fairness requirements

\begin{tabular}{c|c|cccc|cccc}
\hline Index & $C h_{\text {need }}$ & $S R_{\text {FEA }}$ & $S R_{s}$ & $S R_{\text {Thre }}$ & Validation & $F I_{\text {FEA }}$ & $F I_{s}$ & $F I_{\text {Thre }}$ & Validation \\
\hline TUMOCA\#1 & 3 & 0.000 & 0.000 & 0.0 & $\checkmark$ & {$[0.897,0.911]$} & 0.895 & 0.7 & $\checkmark$ \\
TUMOCA\#2 & 4 & 0.000 & 0.000 & 0.0 & $\checkmark$ & {$[0.991,0.991]$} & 0.983 & 0.7 & $\checkmark$ \\
TUMOCA\#3 & 4 & 0.000 & 0.000 & 0.0 & $\checkmark$ & {$[0.975,0.980]$} & 0.977 & 0.7 & $\checkmark$ \\
\hline
\end{tabular}

available to the algorithm, the TUMOCA algorithm also includes the solution using three channels into the new solution set. The TUMOCA algorithm finds a set of feasible solutions instead of one feasible solution. Third, the prediction of goodput and fairness from the fitness function embedded in the TUMOCA algorithm is very close to the simulation results.

Table 5.13: The solution set for a $D=800 \mathrm{~m}$ grid topology with six available channels and goodput requirements

\begin{tabular}{c|c|cccc}
\hline Index & $C h_{\text {need }}$ & $A G_{\text {FEA }}$ & $A G_{s}$ & $A G_{\text {Thre }}$ & Validation \\
\hline TUMOCA\#1 & 5 & {$[0.341,0.353]$} & 0.336 & 0.3 & $\checkmark$ \\
TUMOCA\#2 & 6 & {$[0.335,0.353]$} & 0.355 & 0.3 & $\checkmark$ \\
TUMOCA\#3 & 6 & {$[0.397,0.412]$} & 0.410 & 0.3 & $\checkmark$ \\
\hline
\end{tabular}

Table 5.14: The solution set for a $D=800 \mathrm{~m}$ grid topology with six available channels and fairness requirements

\begin{tabular}{c|c|cccc|cccc}
\hline Index & $C h_{\text {need }}$ & $S R_{\text {FEA }}$ & $S R_{S}$ & $S R_{\text {Thre }}$ & Validation & $F I_{\text {FEA }}$ & $F I_{S}$ & $F I_{\text {Thre }}$ & Validation \\
\hline TUMOCA\#1 & 5 & 0.000 & 0.000 & 0.0 & $\checkmark$ & {$[0.977,0.979]$} & 0.973 & 0.8 & $\checkmark$ \\
TUMOCA\#2 & 6 & 0.000 & 0.000 & 0.0 & $\checkmark$ & {$[0.974,0.977]$} & 0.977 & 0.8 & $\checkmark$ \\
TUMOCA\#3 & 6 & 0.000 & 0.000 & 0.0 & $\checkmark$ & {$[0.961,0.967]$} & 0.957 & 0.8 & $\checkmark$ \\
\hline
\end{tabular}


In the next set of results, a higher QoS requirements is set as $S R_{\text {Thre }}=$ $0.0, F I_{\text {Thre }}=0.8$, and $A G_{\text {Thre }}=0.3$ by using six channels. Tables 5.13 to 5.14 list the solution set from the TUMOCA algorithm with six channels. First, the TUMOCA algorithm starts to find new solutions to meet the higher QoS requirement. The simulation results show that all solutions meet the predefined QoS requirement and the prediction from the fitness function is accurate. In addition, the main difference from existing CA algorithms is that the TUMOCA algorithm meets the desired outcome of finding multiple feasible solutions.

Besides the examples listed here, the TUMOCA algorithm has been validated with different sizes of grid topologies ranging from $200 \mathrm{~m}$ to $900 \mathrm{~m}$ and the maximum channel number ranging from three to twelve non-overlapped channels with different QoS requirements. The simulation results in Appendix F.1 show that TUMOCA algorithm performs well in random topologies. Overall, all the channel allocations from TUMOCA are feasible to meet the predefined QoS requirements.

\subsection{A Traffic-aware Multi-objective Channel As- signment Algorithm For Optimising Fairness and Goodput}

This section develops a traffic-aware multi-objective CA (TAMOCA) algorithm based on the TUMOCA algorithm in Section 5.2. The TAMOCA algorithm aims to improve fairness and goodput considering traffic load.

Similar to the TUMOCA algorithm, the goal of this TAMOCA algorithm is to find a set of feasible solutions of channel allocation to meet a predefined QoS requirements for a given IEEE 802.11 WBN. The predefined performance requirements include fairness requirements as starvation link ratio and Jain's Index, and goodput requirement as average goodput. 
The next sections introduce the design of the TAMOCA algorithm and then validate it through simulation.

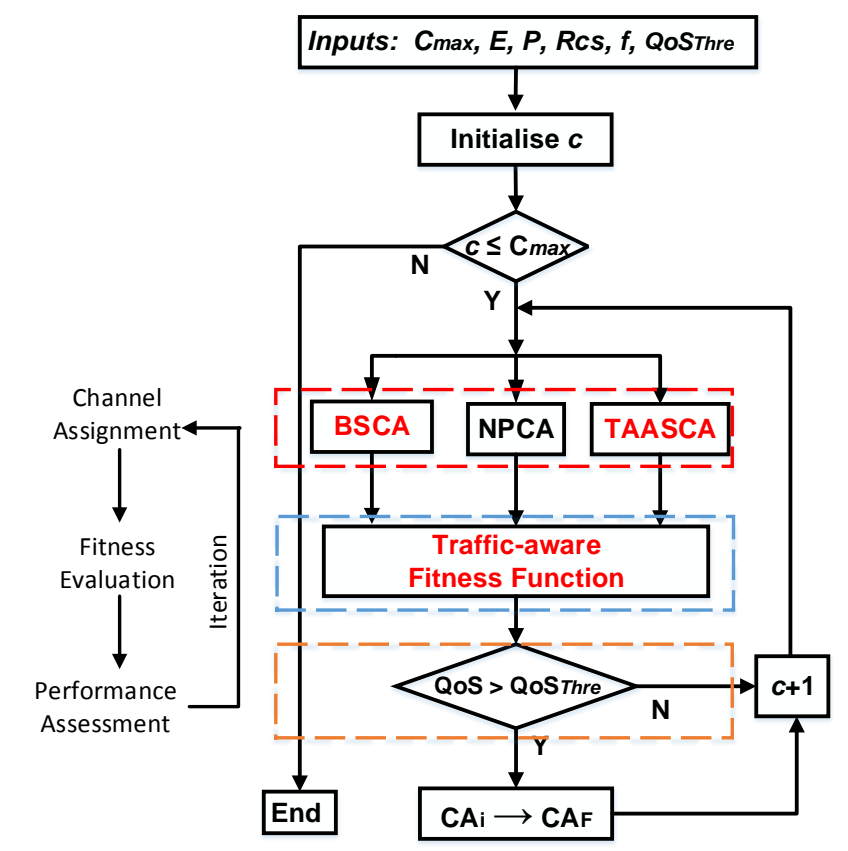

Figure 5.7: The logic flow of the TAMOCA algorithm

\subsubsection{The Design of Traffic-aware Multi-objective Channel Assignment Algorithm}

The TAMOCA algorithm follows the same idea of the multi-objective CA frame with three main components in Figure 5.5. The inputs of the TAMOCA Algorithm are link set $E$, node position $P$, maximum channel number $C_{\text {max }}$, traffic demand $f(i)$, and predefined QoS threshold $Q o S_{\text {Thre }}$. The output of the TAMOCA Algorithm is a set of channel allocations that meet the $Q o S_{\text {Thre }}$ and the performance indicators of each channel allocation.

The logic flow and the pseudo code of the TAMOCA algorithm are shown in Figure 5.7 and Algorithm 12. The structure of TAMOCA is simi- 
lar to that of TUMOCA, that is, to find the feasible solutions by using iteration. At the beginning, TAMOCA initialises channel number $c$ (see line 2) and then starts the iteration of $c$. For each iteration, TAMOCA runs three CA algorithms with the current value of $c$ and yields three channel allocations that form a set of temporary solution $C A_{\text {temp }}$ in lines $4-7$. Next, TAMOCA runs the traffic-aware fitness function to calculate the performance indicators $Q O S_{F E A}$ line 9. In lines $10-12$, TAMOCA compares $Q o S_{F E A}$ with the pre-defined QoS threshold QoS Thre. If the QoS $S_{F E A}$ is greater than $Q o S_{\text {Thre }}$, TAMOCA accepts the channel allocation as a feasible solution. If not, TAMOCA increases $c$ by 1 in line 13 and goes to the next iteration. TAMOCA stops and output the solution set $C A_{F}$ when $c$ is greater than the maximum channel number $C_{\max }$.

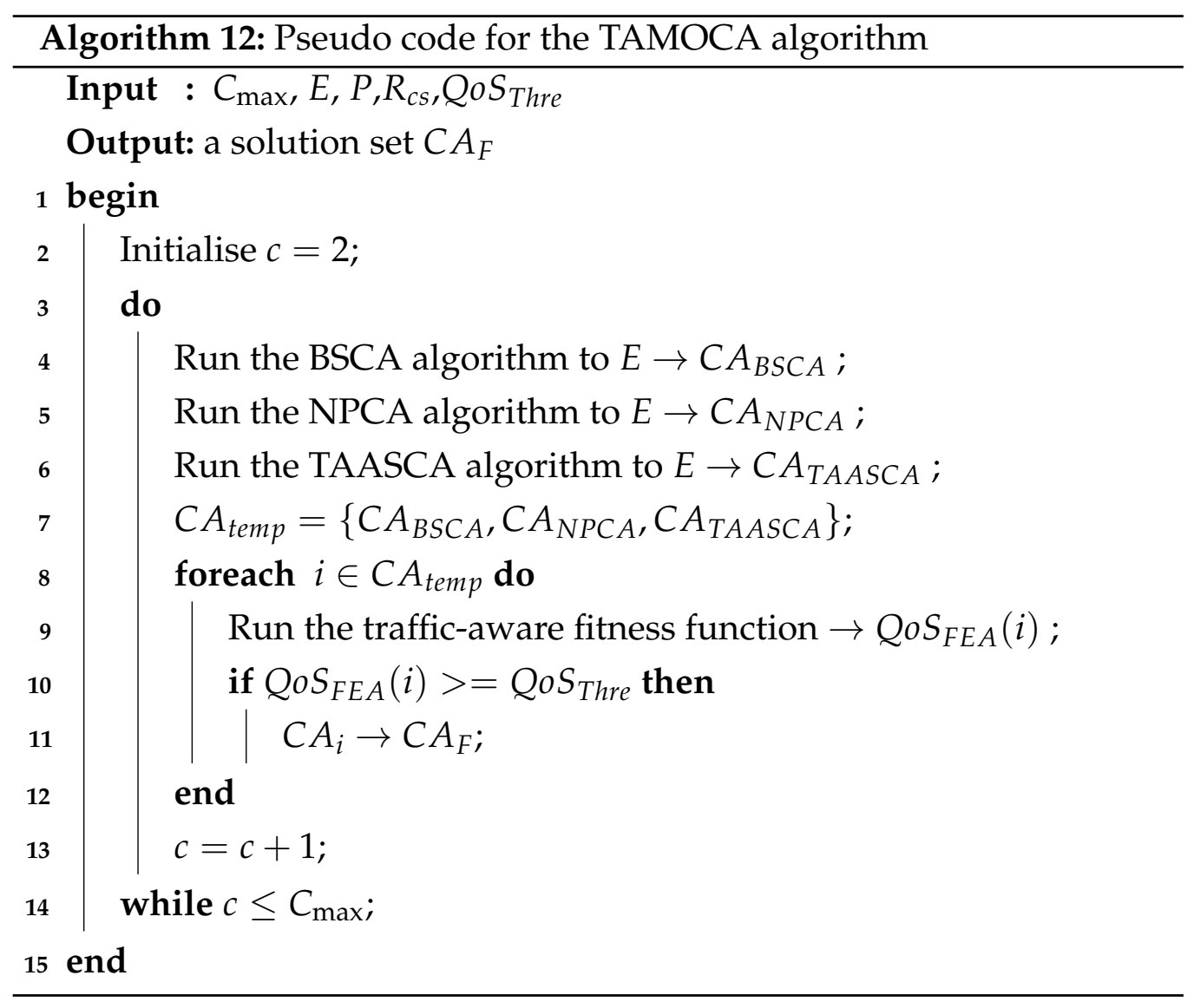


The differences between TUMOCA and TAMOCA are: (i) TAMOCA considers traffic demand $f$, (ii) the integrated CA component in TAMOCA contains traffic-aware CA algorithms BSCA [87] (see Section 4.3.2 on page 114), TAASCA (see Section 4.3.1 on page 112), and NPCA [130] (see Section 4.2.1] on page 84), and (iii) TAMOCA uses the traffic-aware fitness function instead of the traffic-unaware fitness function in TUMOCA.

\subsubsection{Validation of Traffic-aware Multi-objective Channel Assignment Algorithm}

This subsection validates the TAMOCA algorithm through simulation. Similar to the validation of the TUMOCA algorithm, the examples of the TAMOCA algorithm only show the validation in ineffective carrier sensing scenarios. The topology used as an example is a $D=800 \mathrm{~m}, d=50 \mathrm{~m}$, and $D_{t r}=50 \mathrm{~m}$ in Figure 5.4. The simulation runs with Qualnet 5.2 by using the parameters in Table 5.8. The maximum channel number is selected as 4 and 6.

All averages of simulation results shown are reported with confidence interval of $95 \%$ with the range from $[3.6,16.962]$ kbps under the assumption that the averages are normally distributed.

As TAMOCA algorithm is a traffic-aware $\mathrm{CA}$ algorithm, the validation will be conducted in heavy traffic condition $(f(i)=0.8)$ and medium traffic condition $(f(i)=0.4)$ separately.

\subsubsection{Validation of Traffic-aware Multi-objective Channel Assignment in Heavy Traffic Condition $(f(i)=0.8)$}

This subsection validates the TAMOCA algorithm by selecting the traffic demand as 0.8 that is the normalised traffic demand with respect to maximum net bandwidth.

First, the predefined QoS requirements is set as $S R_{\text {Thre }}=0.0, F I_{\text {Thre }}=$ 0.7 , and $A G_{\text {Thre }}=0.2$. Tables 5.15 to 5.16 provides the list of the solu- 
tion set from the TAMOCA algorithm with goodput and fairness requirements using four channels respectively. The $C h_{\text {need }}$ column represents the channel count needed in the corresponding allocation. The "Validation" column shows whether the solution in the row meets the corresponding requirement.

Table 5.15: The solution set for a $D=800 \mathrm{~m}$ grid topology with four available channels and goodput requirements $(f(i)=0.8)$

\begin{tabular}{c|c|cccc}
\hline Index & $C h_{\text {need }}$ & $A G_{F E A}$ & $A G_{s}$ & $A G_{\text {Thre }}$ & validation \\
\hline TAMOCA\#1 & 3 & {$[0.229,0.235]$} & 0.223 & 0.2 & $\checkmark$ \\
TAMOCA\#2 & 4 & {$[0.224,0.235]$} & 0.218 & 0.2 & $\checkmark$ \\
TAMOCA\#3 & 4 & {$[0.285,0.294]$} & 0.277 & 0.2 & $\checkmark$ \\
\hline
\end{tabular}

Table 5.16: The solution set for a $D=800 \mathrm{~m}$ grid topology with four available channels and fairness requirements $(f(i)=0.8)$

\begin{tabular}{c|c|cccc|cccc}
\hline Index & $C h_{\text {need }}$ & $S R_{\text {FEA }}$ & $S R_{S}$ & $S R_{\text {Thre }}$ & Validation & $F I_{\text {FEA }}$ & $F I_{S}$ & $F I_{\text {Thre }}$ & Validation \\
\hline TAMOCA\#1 & 3 & 0.000 & 0.000 & 0.0 & $\checkmark$ & {$[0.897,0.911]$} & 0.894 & 0.7 & $\checkmark$ \\
TAMOCA\#2 & 4 & 0.000 & 0.000 & 0.0 & $\checkmark$ & {$[0.991,0.991]$} & 0.983 & 0.7 & $\checkmark$ \\
TAMOCA\#3 & 4 & 0.000 & 0.000 & 0.0 & $\checkmark$ & {$[0.975,0.980]$} & 0.977 & 0.7 & $\checkmark$ \\
\hline
\end{tabular}

TAMOCA yields three solutions using four channels. When the traffic demand is set as $f(i)=0.8$, this ineffective carrier sensing scenario $(D=800 \mathrm{~m})$ still has the potential risk of border effect and flow starvation. Simulation results show that all the solutions from the TAMOCA algorithm meet the pre-defined QoS requirement with accurate prediction from the fitness function. The same as TUMOCA, the TAMOCA algorithm also includes all the feasible solution in the solution set. In addition, the TAMOCA algorithm meets the desired outcome of finding multiple feasible solutions.

In the next set of results, a higher QoS requirement is set as $S R_{\text {Thre }}=$ $0.0, F I_{\text {Thre }}=0.8$, and $A G_{\text {Thre }}=0.3$ by using six channels. Tables 5.17 
Table 5.17: The solution set for a $D=800 \mathrm{~m}$ grid topology with six available channels and goodput requirements $(f(i)=0.8)$

\begin{tabular}{c|c|cccc}
\hline Index & $C h_{\text {need }}$ & $A G_{\text {FEA }}$ & $A G_{s}$ & $A G_{\text {Thre }}$ & validation \\
\hline TAMOCA\#1 & 5 & {$[0.341,0.353]$} & 0.336 & 0.3 & $\checkmark$ \\
TAMOCA\#2 & 6 & {$[0.335,0.353]$} & 0.355 & 0.3 & $\checkmark$ \\
TAMOCA\#3 & 6 & {$[0.397,0.412]$} & 0.410 & 0.3 & $\checkmark$ \\
\hline
\end{tabular}

Table 5.18: The solution set for a $D=800 \mathrm{~m}$ grid topology with six available channels and fairness requirements $(f(i)=0.8)$

\begin{tabular}{c|c|cccc|cccc}
\hline Index & $C h_{\text {need }}$ & $S R_{\text {FEA }}$ & $S R_{S}$ & $S R_{\text {Thre }}$ & Validation & $F I_{\text {FEA }}$ & $F I_{S}$ & $F I_{\text {Thre }}$ & Validation \\
\hline TAMOCA\#1 & 5 & 0.000 & 0.000 & 0.0 & $\checkmark$ & {$[0.977,0.979]$} & 0.973 & 0.8 & $\checkmark$ \\
TAMOCA\#2 & 6 & 0.000 & 0.000 & 0.0 & $\checkmark$ & {$[0.977,0.974]$} & 0.977 & 0.8 & $\checkmark$ \\
TAMOCA\#3 & 6 & 0.000 & 0.000 & 0.0 & $\checkmark$ & {$[0.961,0.967]$} & 0.957 & 0.8 & $\checkmark$ \\
\hline
\end{tabular}

to 5.18 provide the list of the channel allocation from TAMOCA with six channels. Simulation results show that all the solutions from TAMOCA meet the predefined QoS requirements and the prediction from the fitness function is accurate.

\subsubsection{Validation of Traffic-aware Multi-objective Channel Assignment in Medium Traffic Condition $(f(i)=0.4)$}

This subsection validates TAMOCA by selecting the normalised traffic demand with respect to the maximum net bandwidth as 0.4 for all links in a given IEEE 802.11 WBN.

First, the predefined QoS requirements as $S R_{\text {Thre }}=0.0, F I_{\text {Thre }}=0.7$, and $A G_{\text {Thre }}=0.2$. Tables 5.19 and 5.20 provides the list of the solution set from the TAMOCA algorithm and the three CA algorithms embedded in the TAMOCA algorithm with goodput and fairness requirements using 
four channels respectively.

Table 5.19: The solution set for a $D=800 \mathrm{~m}$ grid topology with four available channels and goodput requirements $(f(i)=0.4)$

\begin{tabular}{c|c|cccc}
\hline Index & $C h_{\text {need }}$ & $A G_{F E A}$ & $A G_{s}$ & $A G_{\text {Thre }}$ & Validation \\
\hline TAMOCA\#1 & 3 & {$[0.254,0.318]$} & 0.291 & 0.2 & $\checkmark$ \\
TAMOCA\#2 & 3 & {$[0.229,0.235]$} & 0.222 & 0.2 & $\checkmark$ \\
TAMOCA\#3 & 4 & {$[0.306,0.365]$} & 0.34 & 0.2 & $\checkmark$ \\
TAMOCA\#4 & 4 & {$[0.224,0.235]$} & 0.218 & 0.2 & $\checkmark$ \\
TAMOCA\#5 & 4 & {$[0.285,0.294]$} & 0.276 & 0.2 & $\checkmark$ \\
\hline
\end{tabular}

Table 5.20: The solution set for a $D=800 \mathrm{~m}$ grid topology with four available channels and fairness requirements $(f(i)=0.4)$

\begin{tabular}{c|c|cccc|cccc}
\hline Index & $C h_{\text {need }}$ & $S R_{\text {FEA }}$ & $S R_{S}$ & $S R_{\text {Thre }}$ & Validation & $F I_{\text {FEA }}$ & $F I_{S}$ & $F I_{\text {Thre }}$ & Validation \\
\hline TAMOCA\#1 & 3 & 0.000 & 0.000 & 0.0 & $\checkmark$ & {$[0.858,0.858]$} & 0.861 & 0.7 & $\checkmark$ \\
TAMOCA\#2 & 3 & 0.000 & 0.000 & 0.0 & $\checkmark$ & {$[0.897,0.912]$} & 0.894 & 0.7 & $\checkmark$ \\
TAMOCA\#3 & 4 & 0.000 & 0.000 & 0.0 & $\checkmark$ & {$[0.952,0.966]$} & 0.955 & 0.7 & $\checkmark$ \\
TAMOCA\#4 & 4 & 0.000 & 0.000 & 0.0 & $\checkmark$ & {$[0.991,0.991]$} & 0.983 & 0.7 & $\checkmark$ \\
TAMOCA\#5 & 4 & 0.000 & 0.000 & 0.0 & $\checkmark$ & {$[0.976,0.98]$} & 0.977 & 0.7 & $\checkmark$ \\
\hline
\end{tabular}

For this scenario $(D=800 \mathrm{~m})$ using four channels, the TAMOCA algorithm yields five solutions and simulation results show all solutions meet the desired performance. With the decrease of traffic demand, the capacity contention reduces so that the TAMOCA algorithm finds more suitable solutions.

Besides the examples listed here, we also validate TAMOCA with different sizes of grid topologies ranging from $200 \mathrm{~m}$ to $900 \mathrm{~m}$ and the maximum channel number ranging from three to twelve non-overlapped channels with different QoS requirements. The simulation results in Appendix F.2 show that TAMOCA algorithm performs well in random topologies with heavy and medium traffic demands. Overall, all the channel allocations from TAMOCA are feasible to meet the predefined QoS requirements. 


\subsection{Summary}

This chapter aims to answer the third research question: "How can a channel assignment algorithm be designed to achieve predefined QoS requirements including fairness and average goodput in IEEE 802.11 WBNs?".

Two new fitness functions are designed to evaluate CA algorithms with saturated and unsaturated traffic assumptions. Simulation results show that the proposed fitness functions provide accurate prediction of goodput distribution, average goodput, starvation link ratio, and fairness index with a given channel allocation in IEEE 802.11 WBNs. Compared with existing evaluation metrics of $\mathrm{CA}$ algorithms, the proposed fitness function is more suitable for evaluating the performance of CA algorithms.

A multi-objective joint framework combines the fitness functions and CA algorithms to achieve both fairness and goodput. This framework introduces a novel and flexible approach for optimising CA with multiple objectives.

Applying the multi-objective joint framework, two new multi-objective channel assignment algorithms, TUMOCA and TAMOCA, are designed to achieve desired outcome of fairness and goodput in IEEE 802.11 WBNs. Simulation results show that the proposed TUMOCA and TAMOCA algorithms provide a set of feasible channel allocations with an accurate prediction of performance to meet the predefined QoS requirements. Multiple feasible solutions from TUMOCA and TAMOCA provide useful and flexible information for network planners to plan IEEE 802.11 WBNs with better fairness and goodput. 


\section{Chapter 6}

\section{Conclusions}

This thesis focuses on improving the planning in IEEE 802.11 WBNs. The overall goal is to improve network performance (fairness and goodput) through goodput modelling and optimising channel assignment.

This research started from goodput modelling in Chapter 3 . A unified goodput distribution model is designed to provide link-level prediction of goodput. In Chapter 4. Based on the goodput model, trafficunaware and traffic-aware anti-starvation channel assignment algorithms are designed with a new traffic-aware interference model. These two antistarvation channel assignment algorithms effectively prevent flow starvation and improve fairness under saturated and unsaturated traffic assumptions. To improve fairness and goodput in IEEE $802.11 \mathrm{WBNs}$, in Chapter 5, we designed traffic-unaware and traffic-aware multi-objective channel assignment algorithms combined with new fitness functions that accurately evaluate the performance of a channel allocation. Both trafficunaware and traffic-aware channel assignment algorithms output a set of feasible solutions to achieve the desired fairness and goodput. Simulation results are shown to validate the proposed goodput model, fitness functions, and channel assignment algorithms throughout the whole thesis.

The new goodput model and proposed channel assignment algorithms help improve IEEE 802.11 WBN planning and guide network designers 
with better evaluation of performance and better channel management.

\subsection{Contributions}

This thesis investigates different aspects regarding goodput modelling and channel assignment in IEEE 802.11 WBNs. The contributions presented in this thesis are listed as follows.

1. A new goodput distribution model provides accurate prediction of the link-level goodput in IEEE 802.11 WBNs. Such a goodput model is useful for node placement at the network planning stage and also for optimising interference model, channel assignment, scheduling, and routing with the prediction of the potential performance.

2. A new traffic-aware interference model based on the new goodput model reflects global interference (border effect) and local interference with respect to traffic pattern in IEEE 802.11 WBNs.

3. Traffic-unaware and traffic-aware channel assignment algorithms using the new interference model effectively prevent flow starvation and improve system fairness in IEEE 802.11 WBNs.

4. Traffic-unaware and traffic-aware fitness functions based on the new goodput model predict the potential performance of the output from channel assignment algorithms in a given IEEE 802.11 WBN. These fitness functions are useful for optimising channel assignment algorithms with evaluation of channel allocation on network performance.

5. Traffic-unaware and traffic-aware multi-objective channel assignment algorithms help guide network designers with a set of feasible solutions. These feasible solutions guarantee to achieve the predefined QoS requirements including fairness and average goodput for a given IEEE 802.11 WBN.

The above major contributions are built up on each other towards addressing the research goal in Figure 6.1. The goodput distribution model is the fundamental contribution. The proposed interference model and 


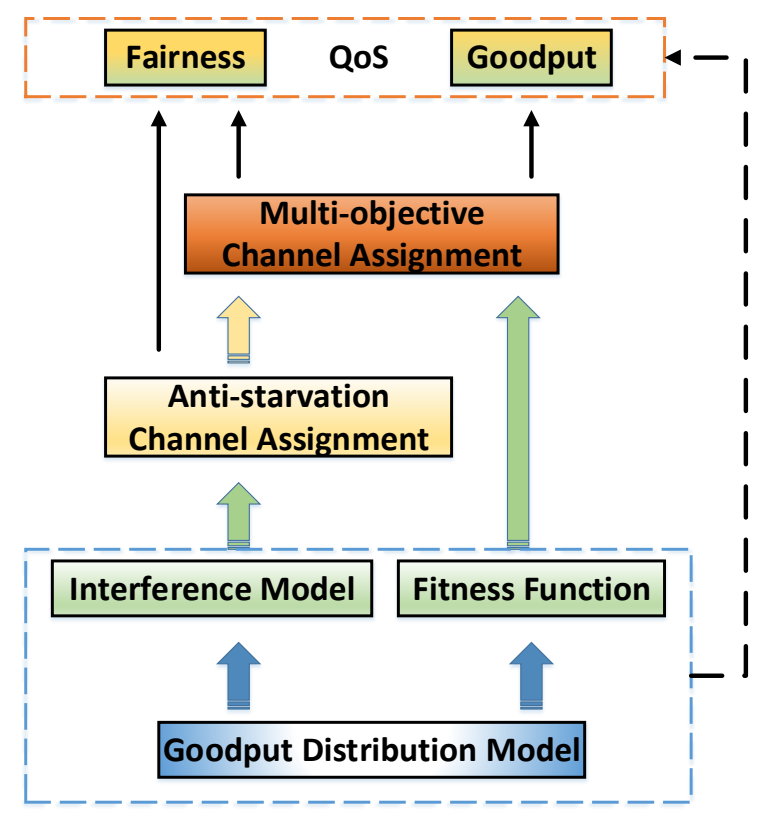

Figure 6.1: Illustration of thesis contributions

the fitness function are based on the goodput distribution model. The anti-starvation channel assignment algorithms based on the interference model improve fairness. Based on the anti-starvation channel assignment algorithms and the fitness function, multi-objective channel assignment algorithms optimise two factors in QoS: fairness and goodput. All the contributions help provide the better management of channel resources, and better fairness and goodput for planning IEEE 802.11 WBNs. Moreover, the contributions can be used to optimise other protocols such as routing and scheduling to meet higher requirements in the future.

\subsection{Future Work}

Due to the scope of this research, there are still some areas for potential extensions and future work. This section will briefly give some directions 
to readers for the study of each chapter.

\subsubsection{Goodput Modelling}

One of the limitations in this research is that the proposed goodput model is based on a linear single-hop topology and all nodes are configured with the identical traffic demand. This is the way to simplify the modelling and reduce the complexity. However, in real-world applications, the multi-hop topology with inequal traffic demands is more common. Therefore, future work could explore how the goodput model predicts the performance in multi-hop topology with different traffic demands in IEEE 802.11 WBNs. In addition, test-bed experiments could be used to further validate our model. With more results, the goodput model could be refined to provide more accurate prediction of link quality in IEEE 802.11 WBNs.

\subsubsection{Interference Model}

The interference model is based on the goodput model. With the improvement of the goodput model, the interference model will also be refined to be more accurate and flexible to multi-hop IEEE 802.11 WBNs with different traffic demands in different links. Moreover, the interference model will consider the accumulated interference from multiple interfering links instead of a pair-wise interference. With the improvement of accuracy, the interference model can help optimise channel assignment algorithms and other approaches with higher performance.

\subsubsection{Channel Assignment with Fairness}

One of the limitations in the anti-starvation channel assignment algorithms is that these two algorithms only considered the feature of multi-channel without considering multi-radio. Future work could add the feature of multi-radio to extend the anti-starvation channel assignment algorithms. 
By considering the features of multi-channel and multi-radio, the antistarvation channel assignment algorithms can be more effective for planning IEEE 802.11 WBNs with better performance. Moreover, with the improvement of the goodput model and the interference model, these antistarvation channel assignment algorithms can be optimised to be more realistic to further improve fairness in multi-hop IEEE 802.11 WBNs. Testbed experiments can be used to validate the anti-starvation channel assignment algorithms and provide feedback to enhance these two algorithms.

\subsubsection{Fitness Function}

The proposed fitness function is designed based on the goodput model. With the improvement of the goodput model, the fitness function will also be refined to be more accurate and flexible to multi-hop IEEE 802.11 WBNs with different traffic demands. Moreover, the fitness function will consider the feature of multi-radio. With the improvement of accuracy and usability, the fitness function can help optimise channel assignment algorithms and other approaches with better performance.

\subsubsection{Channel Assignment with Fairness and Goodput}

Future work could extend the multi-objective channel assignment algorithms to multi-channel and multi-radio IEEE 802.11 WBNs and thus the multi-objective channel assignment algorithms can be more adaptive for optimising IEEE 802.11 WBN planning. With the improvement of the fitness function, these multi-objective channel assignment algorithms can be improved with better accuracy. Since genetic algorithm has been widely used in multi-objective optimisation, Genetic algorithm may be used to solve the optimisation on fairness and goodput in IEEE 802.11 WBNs and validate this idea through simulation. In addition, test-bed experiments can further validate and optimise multi-objective channel assignment. 


\section{Appendix A}

\section{IEEE 802.11 WBN Goodput Patterns with Effective vs. Ineffective Carrier Sensing}

This chapter investigates goodput patterns through simulation in an IEEE 802.11 WBN and discusses the difference between simulation results and expectations from the disk-graph model under effective and ineffective carrier sensing scenarios. Among the goodput models discussed in Chapter 2, the disk-graph model [68] is the simplest model that has been widely used in performance modelling in IEEE 802.11 WBNs [37,75]. The investigation aims to identify how the disk-graph model reflects the behaviour of wireless links in IEEE 802.11 WBNs.

\section{A.1 Simulation Configurations}

To characterise the difference of goodput between disk-graph model and simulation, two typical carrier sensing scenarios are selected with structured linear topologies and two types of traffic demands. Two typical carrier sensing scenarios cover effective and ineffective carrier sensing scenarios. 


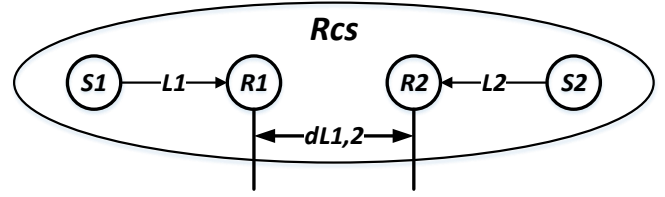

Figure A.1: The two-link scenario representing effective CSMA when two links are within each other's carrier sensing range

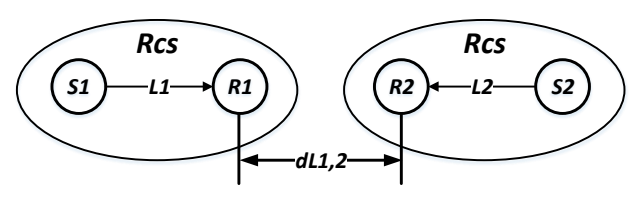

Figure A.2: The two-link scenario representing effective CSMA when two links are out of each other's carrier sensing range

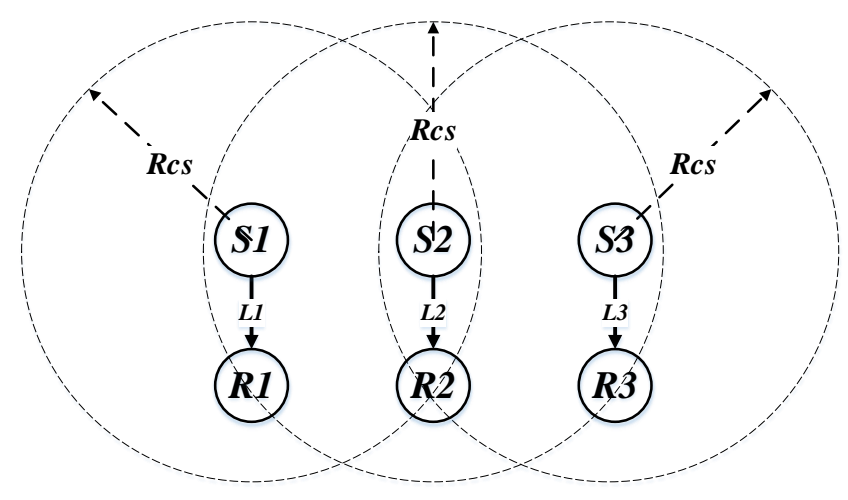

Figure A.3: The three-link scenario representing ineffective CSMA with border effect

The effective carrier sensing scenario is selected with a two-link scenario (see Figures A.1 and A.2), which is a building block of any WBNs. In Figure A.1. four nodes $S 1, R 1, S 2$, and $R 2$ are within each other's carrier sensing range. In Figure A.2, two links $L 1$ and $L 2$ are within each other's carrier sensing range. The ineffective carrier sensing scenario is selected with a three-link scenario (see Figure A.3) that can be regarded 
as the combination of three two-link scenarios and includes many typical issues in IEEE 802.11 WBNs such as border effect.

Two types of traffic demands considered include saturated and unsaturated traffic demands. Saturated traffic represents heavy traffic across the IEEE 802.11 WBN (e.g. dense urban area, providing Internet access via an IEEE 802.11 WBN) and unsaturated traffic represents the heavy, medium and light traffic across an IEEE 802.11 WBN (e.g. sensor network information exchange, machine-to-machine communication and traffic from the Internet of Things). These two scenarios and two traffic demands try to cover the characteristics of various application scenarios in IEEE 802.11 WBNs.

Table A.1: Simulation configuration parameters

\begin{tabular}{l|l}
\hline Parameter Name & Value \\
\hline Transmission Power & $18 \mathrm{dBm}$ \\
\hline Receiver Sensitivity & $-78 \mathrm{dBm}$ \\
\hline Path Loss Model & Two-Ray \\
\hline Shadowing and Fading Model & None \\
\hline Physical Layer & IEEE 802.11 a \\
\hline Data Rate & $24 \mathrm{Mbps}$ \\
\hline MAC Layer & PCS \\
\hline Routing & Static Routing \\
\hline Transportation Layer & UDP \\
\hline Packet Size & 1500 Bytes \\
\hline Inter-packet Interval for Saturated Traffic & $0.5 \mathrm{~ms}$ \\
\hline Inter-packet Interval for Unsaturated Traffic & $0.5-3.45 \mathrm{~ms}$ \\
\hline
\end{tabular}

All the simulations are conducted in QualNet 5.2. Table A.1 lists the main configuration parameters. Note that in this thesis, only physical carrier sensing (PCS) is used without using virtual carrier sensing. The theoretical maximum transmission range $D_{t r}^{\max }$ in this simulation is approximately $207 \mathrm{~m}$. This value is calculated by QualNet's radio range utility with the simulation scenario as input. Moreover, physical carrier sensing 
range $R_{c S}$ is defined by a triplet consisting of (i) the minimum receiver sensitivity of $-78 \mathrm{dBm}$, (ii) maximum transmission power of $18 \mathrm{dBm}$ (based on Alcatel Lucent WaveLAN card), and (iii) the two-ray propagation model, which yields the distance of $515 \mathrm{~m}$.

Part of the results in the following subsections appears in our previous work [114,116]. Simulation results shown in this section are averages from 100 randomly seeded simulation runs. All averages shown are reported with confidence interval of $95 \%$ with the range of $[2.746,34.359] \mathrm{kbps}$ under the assumption that the averages are normally distributed.

The next sections will discuss the simulation results and how the results match up with the predictions from the disk-graph model under effective and ineffective carrier sensing conditions separately.

\section{A.2 A Disk-graph Model Accurately Character- izes Goodput Distribution Under Effective Carrier Sensing in IEEE 802.11 WBNs}

This subsection shows that the disk-graph model accurately characterizes goodput distribution in effective carrier sensing by using the simulation results in a two-link scenario. The two-link scenario is a simple singleradio single-channel IEEE $802.11 \mathrm{WBN}$ scenario (see Figures A.1 and A.2. There are two pairs of nodes denoted by $(S 1, R 1)$ and $(S 2, R 2)$ in the network and communicating pairs are connected through wireless links $L 1$ and $L 2$ respectively. A constant bit rate (CBR) traffic generator sending packets at $24 \mathrm{Mbps}$ encapsulated with UDP is chosen as the application for the senders. All nodes are configured with identical parameters and wireless links $L 1$ and $L 2$ utilise the same channel.

For effective carrier sensing scenario, two links $L 1$ and $L 2$ are either within each other's carrier sensing range or out of each other's carrier sensing range. As links $L 1$ and $L 2$ are like "mirror" links in the two-link 
scenario, the aggregated goodput of links $L 1$ and $L 2$ is used in the analysis instead of individual goodput for both saturated and unsaturated traffic demands.

\section{A.2.1 Saturated Traffic Demand}

Our previous paper [114] studied the two-link scenario with saturated traffic demand and reported: (i) For distance $D_{t r}=50 m<0.5 D_{t r}^{\max }$, very few collisions occur; (ii) when two links are within each other's carrier sensing range, they share channel capacity equally; (iii) when two links are out of each other's carrier sensing range, they appear as two separate networks.

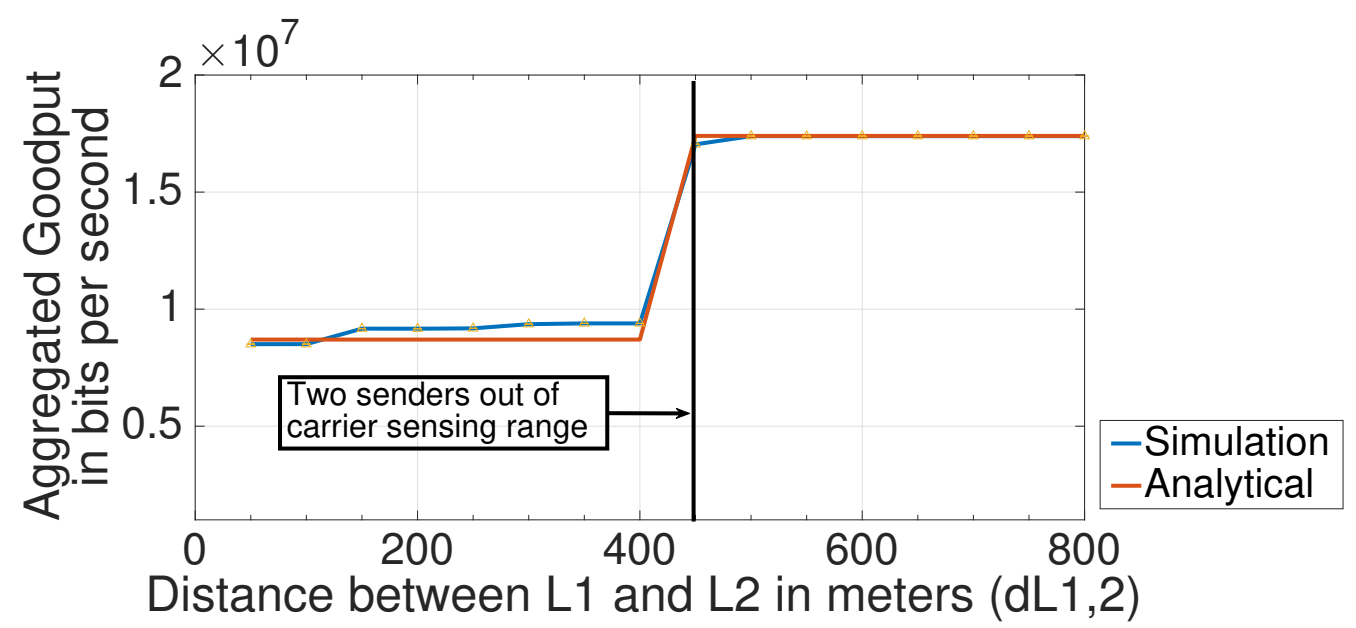

Figure A.4: Comparison of aggregated goodput between simulation and disk-graph model for $D_{t r}=50 \mathrm{~m}$ (PCS mechanism)

An example using PCS mechanism is used to help readers understand the above findings in Figure A.4. PCS mechanism refers to physical carrier sensing mechanism without using virtual carrier sensing mechanism. In Figure A.4, the $\mathrm{X}$ axis refers to the distance between two links $L 1$ and $L 2$ denoted by $d L 1,2$ (see Figure A.1) and $Y$ axis presents the aggregated 
goodput of links L1 and L2. The two lines in the figure refer to the simulation result and the expectation from the disk-graph analytical model.

The expectation from disk-graph analytical model is that within carrier sensing range, two links $L 1$ and $L 2$ fairly share the channel capacity. Out of each other's carrier sensing range, two links $L 1$ and $L 2$ occupy the channel capacity. The simulation results match with the expected goodput pattern form disk-graph model. The aggregated goodput over a range of $d L 1,2$ shows two distinct trends in Figure A.4, a step-like goodput response is observed with increasing $d L 1,2$. We notice that the simulation results are slightly higher than the prediction from the analytical model. It implies that even all nodes are within each other's carrier sensing range, carrier sensing mechanism may allow concurrent transmissions for a short term that leads to a slightly higher goodput in the simulation than the analytical prediction.

\section{A.2.2 Unsaturated Traffic Demand}

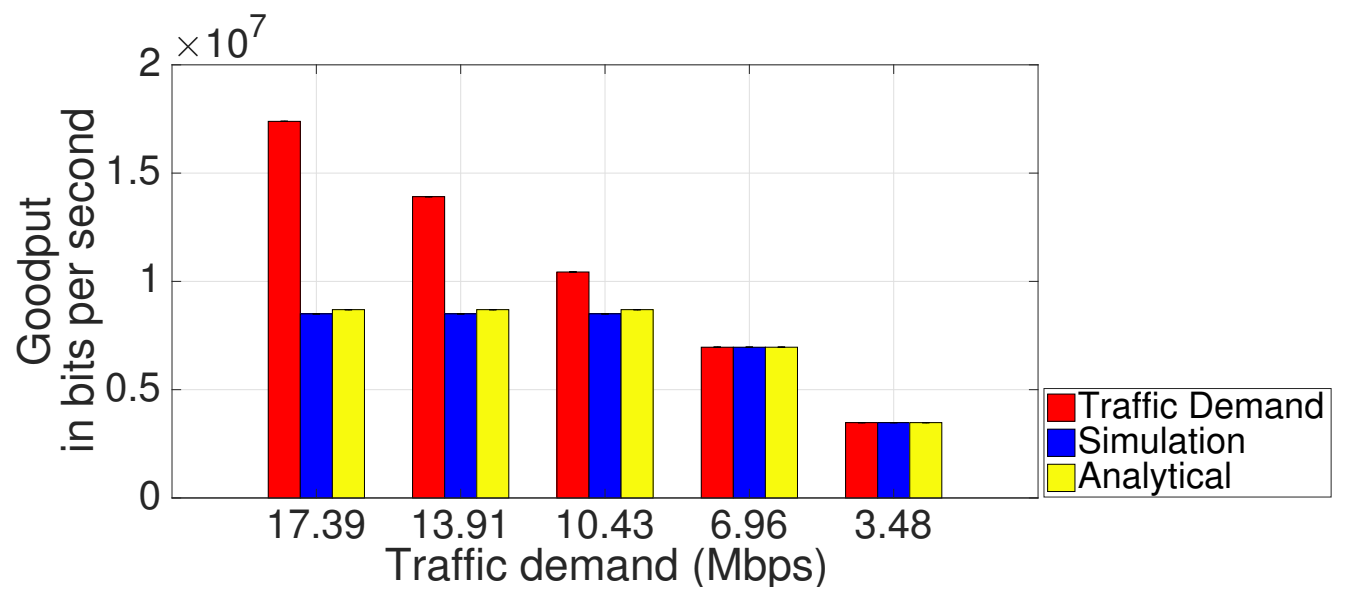

Figure A.5: Comparison of aggregated goodput between simulation and disk-graph model with unsaturated traffic demands for $d_{L 1,2}=100 \mathrm{~m}$ in the two-link scenario

For unsaturated traffic demand in two-link scenario, the focus is the 
goodput pattern when the two links are within each other's carrier sensing range. Hence, the topology in Figure A.1 is configured as where the distance between links $L 1$ and $L 2, d_{L 1,2}=100 \mathrm{~m}$. Five traffic demands are selected for links $L 1$ and $L 2,17.39 \mathrm{Mbps}, 13.91 \mathrm{Mbps}, 10.43 \mathrm{Mbps}, 6.96 \mathrm{Mbps}$, and $3.48 \mathrm{Mbps}$ calculated at application layer. These five traffic demands are not greater than $17.39 \mathrm{Mbps}$ that is the net capacity excluded overhead calculated from simulation results. The traffic demands (plotted as red bars) is compared with the actual aggregated goodput of links L1 and L2 (plotted as blue bars) and the prediction from the disk-graph analytical model (plotted as yellow bars) in Figure A.5.

The expectation of the disk-graph model is that when the sum of the traffic demands of two links exceeds the net capacity, they will share the capacity equally, while two links can achieve the desired goodput if the sum of the traffic demands on both of them are less than the net capacity. These simulation results match with the expectation from the disk-graph model.

In this figure, when the sum of the traffic demands of these two links L1 and $L 2$ exceeds the channel capacity, both links cannot achieve their traffic demands and have to share the channel capacity fairly (see $17.39 \mathrm{Mbps}$, 13.91 Mbps, $10.43 \mathrm{Mbps}$ in Figure A.5). For example, when the traffic demand rate of links $L 1$ and $L 2$ is $13.91 \mathrm{Mbps}$, the sum of the traffic demand of these two links is $27.82 \mathrm{Mbps}$ that exceeds the net capacity. Because links L1 and L2 are within each other's carrier sensing range, each link has to share the channel capacity and can only achieve about $8.5 \mathrm{Mbps}$ goodput.

When the sum of the traffic demands of these two links is less than the channel capacity 17.39 Mbps (see 6.96 Mbps, and 3.48 Mbps in Figure A.5), each link achieves their traffic demands. For example, when the traffic demand rate of links $L 1$ and $L 2$ is $6.96 \mathrm{Mbps}$, the sum of the traffic demand of these two links is $13.92 \mathrm{Mbps}$ that is less than the net capacity and both links can achieve their traffic demands. 


\section{A.3 A Disk-graph Model Fails to Characterize Good- put Distribution in Ineffective Carrier Sens- ing in WBNs}

This subsection will discuss the disk-graph model fails to characterize the goodput distribution in ineffective carrier sensing scenario. The effective carrier sensing scenario is changed into the ineffective carrier sensing scenario by adding a third link to the two-link scenario (see Figure A.3). This scenario is a typical example for border effect where links $L 1$ and $L 3$ are the two border links that are beyond each other's carrier sensing range. Link $L 2$ is in the middle and within the carrier sensing range of both links $L 1$ and $L 3$.

\section{A.3.1 Saturated Traffic Demand}

For saturated traffic demand in ineffective carrier sensing scenario, our previous paper [116] reported that when the distance between two border links exceeds each other's carrier sensing range, border effect exists and causes flow starvation in the middle link.

An exampl in Figure A.6 explains border effect and flow starvation in which the disk-graph model fails to characterize the goodput pattern. In the three-link scenario (see Figure A.3), as links L1 and L3 are out of each other's carrier sensing range, they do not interfere with each other. Two pairs of links $L 1$ and $L 2$, links $L 2$ and $L 3$ are still within each other's carrier sensing range. From the perspective of link L2, links L1 and L3 are with its carrier sensing range. According to the disk-graph model, the expectation is that three links will share the channel capacity equally (see Figure A.6). However, the simulation results show a different pattern in that two border links L1 and L3 utilise the spatial resource to achieve highest goodput based on the sacrifice of the goodput in link L2. These results do not match with the expection from the disk-graph model. 


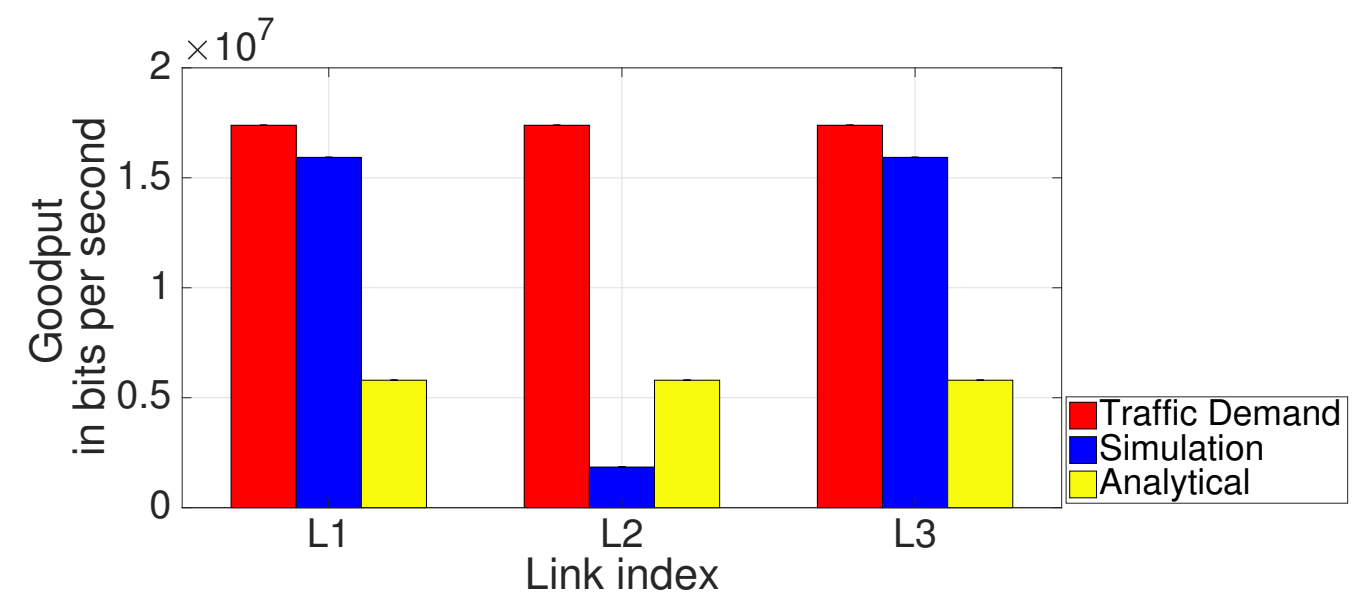

Figure A.6: Comparison of goodput between simulation and disk-graph model in three-link Scenario $D=800 \mathrm{~m}$ with saturated traffic demands

\section{A.3.2 Unsaturated Traffic Demand}

For unsaturated traffic demand in the three link scenario, five traffic demands are selected for the links $L 1, L 2$ and $L 3$, as $17.39 \mathrm{Mbps}, 13.91 \mathrm{Mbps}$, $10.43 \mathrm{Mbps}$, 6.96 Mbps, and $3.48 \mathrm{Mbps}$, which are lower than the net capacity $17.39 \mathrm{Mbps}$.

We list the comparison between traffic demands and the actual goodput of three links L1, L2 and L3 in Figure A.7. The $X$ axis denotes the traffic demand rate of each link, the red bar represents the traffic demand rate and the blue, brown, and pink bars represent the actual goodput of the links $L 1, L 2$ and $L 3$ respectively. The yellow bar refers to the prediction from the disk-graph analytical model.

The expectation of the disk-graph model is that three links will share the channel capacity from the perspective of the middle link L2. Therefore, when the sum of the traffic demands of the three links exceed the net capacity, these three links will share the capacity equally. If the sum of the traffic demands of these three links is less than the net capacity, these links achieve the desired goodput. 


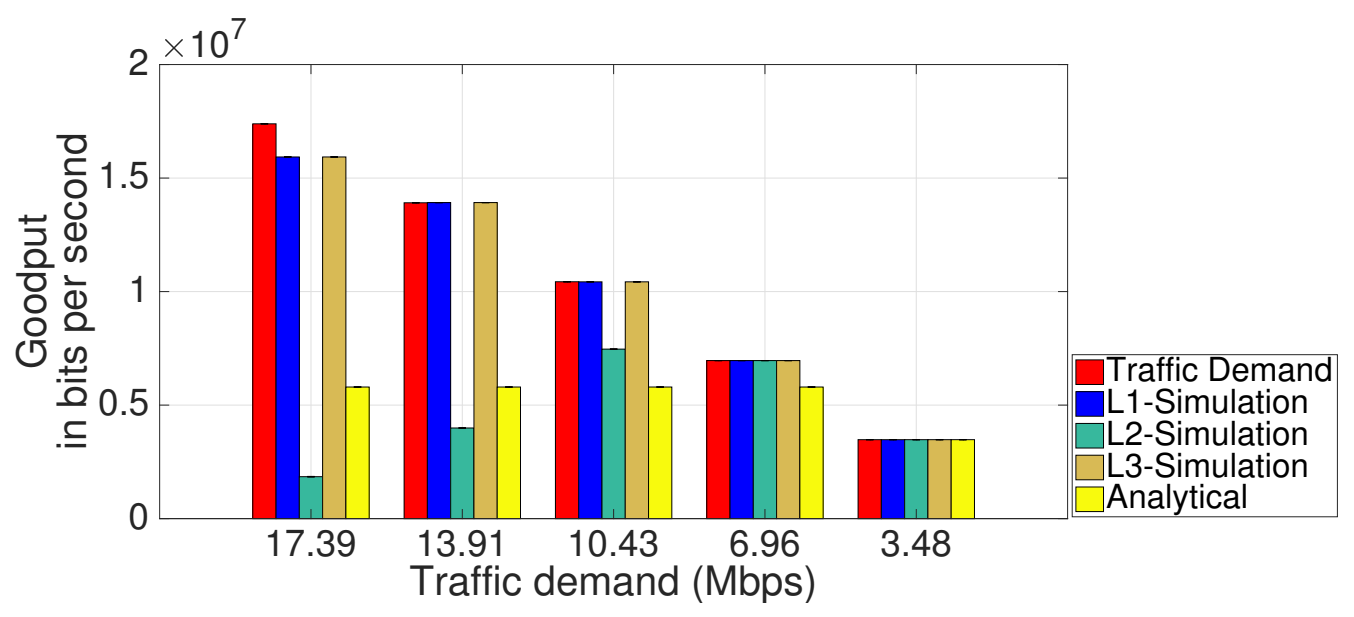

Figure A.7: Comparison of goodput between simulation and disk-graph model in three-link scenario $D=800 \mathrm{~m}$ with unsaturated traffic demands

In Figure A.7, simulation results do not totally match with the prediction from the disk-graph model. When the sum of the traffic demands of two border links $L 1$ and $L 3$ exceeds the channel capacity, starvation exists at link $L 2$ and two border links $L 1$ and $L 3$ occupy the channel capacity. For example, when the traffic demand rate of three links is $13.91 \mathrm{Mbps}$, the sum of the traffic demand of three links is $41.73 \mathrm{Mbps}$ that exceeds the net capacity. The two border links $L 1$ and $L 3$ achieve the desired goodput while the middle link $L 2$ achieves very little goodput. In addition, the sum of three links exceed the channel capacity that implies spatial reuse exists in ineffective carrier sensing scenarios. These goodput patterns are unexpected from the disk-graph model.

When the sum of the traffic demands of the two border links $L 1$ and L3 is less than the channel capacity (see $3.48 \mathrm{Mbps}$ in Figure A.7), starvation does not exist and these three links achieve the traffic demand. These simulation results match the expectation from the disk-graph model. 


\section{A.4 Summary}

Based on the above observations, the goodput patterns in effective carrier sensing scenarios match with the expectations from the disk-graph model. When all nodes are within each other's carrier sensing range, these nodes share the channel capacity fairly. When all nodes are out of each other's carrier sensing range, each node occupies the whole channel capacity.

However, some simulation results in ineffective carrier sensing scenarios show an unexpected goodput pattern from the disk-graph model. According to the disk-graph model, links within the carrier sensing range of a link should share channel capacity equally and the sum of goodput of the links in any interference set is below 1 . However, this conclusion does not hold in the scenarios with border effect and flow starvation. Hence, the disk-graph model cannot explain the flow starvation in the middle link and the interaction between border links and middle link.

In a larger network, the main reason for unfairness between border links and middle links is that the border links and the middle links sense the channel state differently due to the layout of IEEE 802.11 WBNs and CSMA mechanism. The border links have fewer neighbouring links than the links between borders and they are likely to transmit more packets. The transmission attempt of links in the middle have to contend with the border links and are more likely to back off until the channel is released by the border links. Therefore, this asymmetric back off leads to unfair sharing of channel among the links in IEEE 802.11 WBNs and some middle links may starve.

In all, the disk-graph model is a simple model that predicts the goodput pattern in effective carrier sensing scenarios but it fails to predict the goodput pattern in ineffective carrier sensing scenarios. 
180APPENDIX A. IEEE 802.11 WBN GOODPUT PATTERNS WITH CSMA 


\section{Appendix B}

\section{Proof of The Proposed Goodput Distribution Model}

\section{B.1 Goodput For Effective Carrier Sensing with Saturated Traffic Assumption}

Proof. Let $N$ denote the number of links in a given IEEE 802.11 WBN and $f(i)$ the normalised traffic demand of a given link $i$ with respect to the maximum net capacity.

For $N=1$, then there is one link $L_{1}$ in the IEEE 802.11 WBN. If the traffic demand of this link $f\left(L_{1}\right)=1$, then $L_{1}$ achieves the full capacity:

$$
G\left(L_{1}\right)=\frac{1}{N}=1 \text {. }
$$

For $N=2$, there are two links $L_{1}$ and $L_{2}$ in the IEEE 802.11 WBN. If the traffic demand of these two links is $f\left(L_{1}\right)=f\left(L_{2}\right)=1$, the sum of the traffic demands of these two links exceeds the capacity $f\left(L_{1}\right)+f\left(L_{2}\right)>1$, then these two links have to share the capacity and achieve goodput:

$$
G\left(L_{1}\right)=G\left(L_{2}\right) \text { and } G\left(L_{1}\right)+G\left(L_{2}\right)=1 \text {. }
$$

Therefore, $G\left(L_{i}\right)=\frac{1}{N}, \forall i \in N$.

For $N=k$, there are $k$ links $L_{1}, \ldots$ and $L_{k}$ in the IEEE 802.11 WBN. If the 
traffic demand of these $k$ links is $f\left(L_{1}\right)=\ldots=f\left(L_{k}\right)=1$, the sum of the traffic demands of these $k$ links exceeds the capacity $\sum_{j \in k} f(j)>1$, then these $k$ links have to share the capacity and achieve:

$$
G\left(L_{1}\right)=G\left(L_{1}\right) \ldots=G\left(L_{k}\right) \text { and } \sum_{j \in k} f(j)>1 .
$$

Therefore, we have $G\left(L_{i}\right)=\frac{1}{N}, \forall i \in N$.

By induction on $N$, the goodput for a given link for saturated traffic demand in effective carrier sensing scenario in Def. 1.

\section{B.2 Pessimistic and Optimistic Goodput Under Ineffective CSMA with Saturated Traffic As- sumption}

Proof. Let $N$ be the number of links in a given IEEE $802.11 \mathrm{WBN}, f(i)$ is the normalised traffic demand of a given link $i$ with respect to the maximum net capacity, IS $(i)$ and $\gamma(i)$ are the independent set and conflict set of a given link $i$ (see Definitions in Section 3.1.1.2 on page 46).

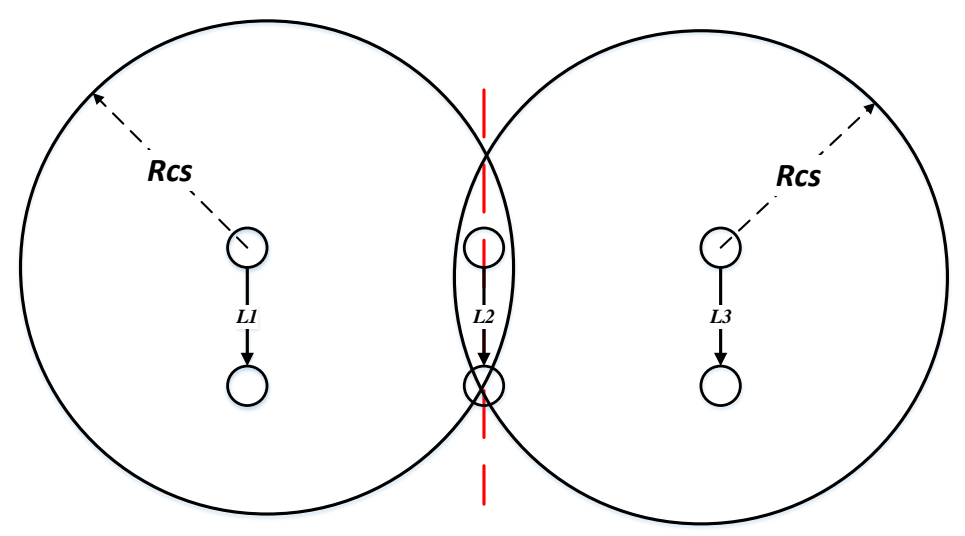

Figure B.1: A three-link IEEE 802.11 WBN

Let $N=3$, there are three links $L_{1}, L_{2}$, and $L_{3}$ in the IEEE 802.11 WBN (see Figure B.1). By Def. 3, the conflict set of each link $i, i \in\{1,2,3\}$ are: 
$\gamma\left(L_{1}\right)=\left\{L_{2}\right\}, \gamma\left(L_{2}\right)=\left\{L_{1}, L_{3}\right\}, \gamma\left(L_{3}\right)=\left\{L_{2}\right\}$, and by Def. 2 2 the independent set of each link $i$ is:

$$
I S\left(L_{1}\right)=\left\{L_{3}\right\}, I S\left(L_{3}\right)=\left\{L_{1}\right\}, I S\left(L_{2}\right)=\{\varnothing\} .
$$

Let the traffic demand of each link be the saturated traffic as $f(i)=1$. As link $L_{2}$ is conflict with links $L_{1}$ and $L_{3}$, we calculate the pessimistic goodput of link $L_{2}$ with the optimistic goodput of links $L_{1}$ and $L_{3}$. Because $L_{2}$ has no link in its independent set the goodput is zero i.e. $G_{P}\left(L_{2}\right)=0.0$, it follows that links $L_{1}$ and $L_{3}$ will occupy full capacity:

$$
\begin{aligned}
G_{O}\left(L_{1}\right) & =\frac{\left|I S\left(L_{1}\right)\right|}{\left|I S\left(L_{1}\right)\right|+\left|I S\left(L_{2}\right)\right|}, \\
G_{O}\left(L_{3}\right) & =\frac{\left|I S\left(L_{3}\right)\right|}{\left|I S\left(L_{3}\right)\right|+\left|I S\left(L_{2}\right)\right|}, \\
G_{O}\left(L_{1}\right)+G_{P}\left(L_{2}\right) & =1 \text { and } G_{O}\left(L_{2}\right)+G_{P}\left(L_{3}\right)=1 .
\end{aligned}
$$

By Def. 7. $\gamma^{G M C}=\left\{L_{1}, L_{3}\right\}$ and $\left|\gamma^{G M C}\right|=2$. By Def. 9 the optimistic goodput of starving link $L_{2}$ is:

$$
G_{O}\left(L_{2}\right)=\alpha \times \frac{\left|\gamma^{G M C}\right|}{N}=0.133 \text { where } \alpha \text { is selected as } 0.2 \text {, }
$$

which implies that starving link achieves a non-zero goodput. Since starving link $L_{2}$ may achieve non-zero goodput but very low goodput, links $L_{1}$ and $L_{3}$ cannot occupy the whole capacity. Thus, the pessimistic goodput of links $L_{1}$ and $L_{3}$ will be :

$$
\begin{aligned}
& G_{P}\left(L_{1}\right)=\frac{\left|I S\left(L_{1}\right)\right|}{\left|I S\left(L_{1}\right)\right|+\left|I S\left(L_{2}\right)\right|} \times\left(1-G_{O}\left(L_{2}\right)\right), \\
& G_{P}\left(L_{3}\right)=\frac{\left|I S\left(L_{3}\right)\right|}{\left|I S\left(L_{2}\right)\right|+\left|I S\left(L_{3}\right)\right|} \times\left(1-G_{O}\left(L_{2}\right)\right) .
\end{aligned}
$$

From the perspective of links $L_{1}$ and $L_{3}$, the sum of goodput in their conflict sets follows: $G_{O}\left(L_{2}\right)+G_{P}\left(L_{1}\right)=1$ and $G_{O}\left(L_{2}\right)+G_{P}\left(L_{3}\right)=1$.

Let $N=4$, there are four links in Figure B.2. By Def. 3, the conflict set of each link $i, i \in\{1,2,3,4\}$ are:

$$
\gamma\left(L_{1}\right)=\left\{L_{2}\right\}, \gamma\left(L_{2}\right)=\left\{L_{1}, L_{3}\right\}, \gamma\left(L_{3}\right)=\left\{L_{2}, L_{4}\right\}, \gamma\left(L_{4}\right)=\left\{L_{3}\right\}
$$

and by Def. 2 the independent set of each link $i$ is: 


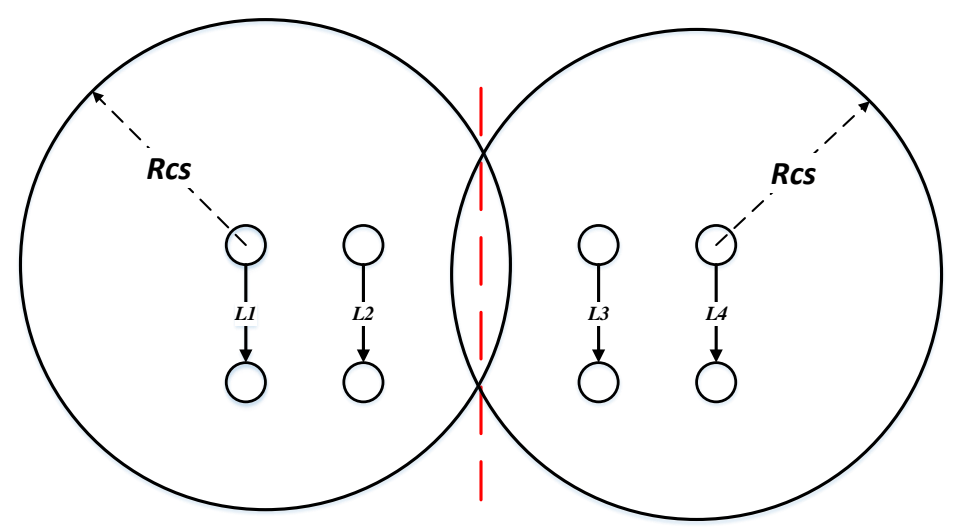

Figure B.2: A four-link IEEE 802.11 WBN

$$
\text { IS }\left(L_{1}\right)=\left\{L_{3}, L_{4}\right\}, I S\left(L_{3}\right)=\left\{L_{1}\right\}, I S\left(L_{3}\right)=\left\{L_{1}\right\}, I S\left(L_{4}\right)=\left\{L_{1}, L_{2}\right\} .
$$

Thus, by Def.(8) no link will be starved because no link is within the carrier sensing range of two border links. It follows that two subgroups $\left\{L_{1}, L_{2}\right\}$ and $\left\{L_{3}, L_{4}\right\}$ occupy full capacity for the optimistic situation because two border links are out of each other's carrier sensing range. In each subgroup, the optimistic goodput of a link will be proportional to the number of links in its independent set and the capacity contention is only considered within the conflict set of border link:

$$
\begin{aligned}
G_{O}\left(L_{1}\right) & =\frac{\left|I S\left(L_{1}\right)\right|}{\left|I S\left(L_{1}\right)\right|+\left|I S\left(L_{2}\right)\right|}, \\
G_{O}\left(L_{2}\right) & =\frac{\left|I S\left(L_{2}\right)\right|}{\left|I S\left(L_{1}\right)\right|+\left|I S\left(L_{2}\right)\right|}, \\
G_{O}\left(L_{1}\right)+G_{O}\left(L_{2}\right) & =1 .
\end{aligned}
$$

Similarly, the goodput for the other two links are:

$$
\begin{aligned}
G_{O}\left(L_{3}\right) & =\frac{\left|I S\left(L_{3}\right)\right|}{\left|I S\left(L_{3}\right)\right|+\left|I S\left(L_{4}\right)\right|}, \\
G_{O}\left(L_{4}\right) & =\frac{\left|I S\left(L_{4}\right)\right|}{\left|I S\left(L_{3}\right)\right|+\left|I S\left(L_{4}\right)\right|}, \\
G_{O}\left(L_{3}\right)+G_{O}\left(L_{4}\right) & =1 .
\end{aligned}
$$


By Def. 7. $\gamma^{G M C}=\left\{L_{1}, L_{4}\right\}$ and $\gamma^{G M C}=2$. Since in this four-link WBN, there is no starving link. So the pessimistic goodput of links are the same as their optimistic goodput following the same assumption that goodput is proportional to the number of links in the independent set.

For left border link $L_{1}$, the pessimistic goodput follows $G_{P}\left(L_{1}\right)+G_{P}\left(L_{2}\right)=$ 1 and is:

$$
G_{P}\left(L_{1}\right)=\frac{\left|I S\left(L_{1}\right)\right|}{\left|I S\left(L_{1}\right)\right|+\left|I S\left(L_{2}\right)\right|},
$$

For link $L_{2}, \gamma\left(L_{2}\right)=\left\{L_{1}, L_{3}\right\}$. The pessimistic goodput follows $G_{P}\left(L_{1}\right)+$ $G_{P}\left(L_{2}\right)+G_{P}\left(L_{3}\right)=1$ and is:

$$
G_{P}\left(L_{2}\right)=\frac{\left|I S\left(L_{2}\right)\right|}{\left|I S\left(L_{1}\right)\right|+\left|I S\left(L_{2}\right)+\right| I S\left(L_{3}\right) \mid} .
$$

Similarly, the pessimistic goodput of links $L_{3}$ and $L_{4}$ are:

$$
\begin{aligned}
G_{P}\left(L_{3}\right) & =\frac{\left|I S\left(L_{3}\right)\right|}{\left|I S\left(L_{3}\right)\right|+\left|I S\left(L_{2}\right)\right|+\left|I S\left(L_{4}\right)\right|}, \\
G_{P}\left(L_{4}\right) & =\frac{\left|I S\left(L_{4}\right)\right|}{\left|I S\left(L_{3}\right)\right|+\left|I S\left(L_{4}\right)\right|} .
\end{aligned}
$$

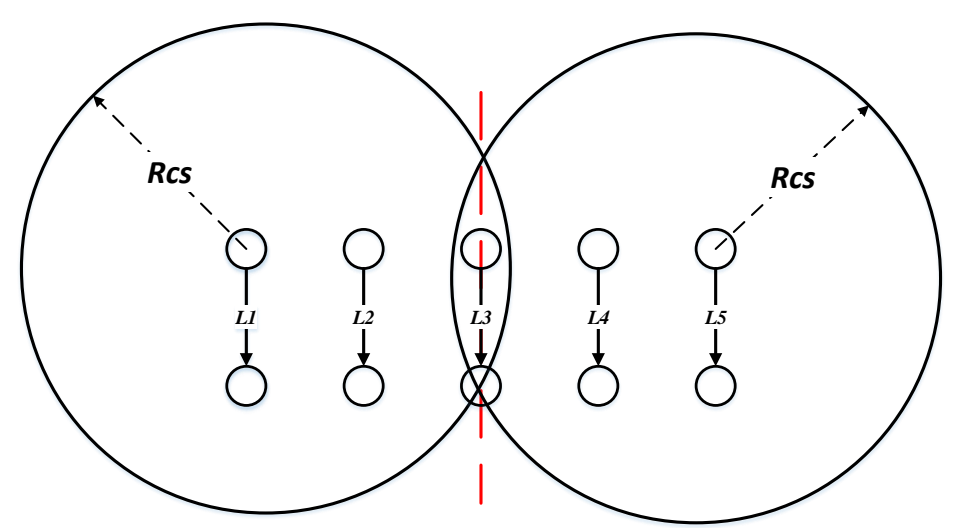

Figure B.3: A five-link IEEE 802.11 WBN

Let $N=5$, there are five links in Figure B.3. By Def. 3 , the conflict set of each link $i, i \in\{1,2,3,4,5\}$ are:

$$
\begin{aligned}
& \gamma\left(L_{1}\right)=\left\{L_{2}, L_{3}\right\}, \gamma\left(L_{2}\right)=\left\{L_{1}, L_{3}, L_{4}\right\}, \gamma\left(L_{3}\right)=\left\{L_{1}, L_{3}, L_{4}, L_{5}\right\}, \gamma\left(L_{4}\right)= \\
& \left\{L_{2}, L_{3}, L_{5}\right\}, \gamma\left(L_{5}\right)=\left\{L_{3}, L_{4}\right\} .
\end{aligned}
$$


and by Def. 2, the independent set of each link $i$ is:

$$
\begin{aligned}
& I S\left(L_{1}\right)=\left\{L_{4}, L_{5}\right\}, I S\left(L_{2}\right)=\left\{L_{5}\right\}, I S\left(L_{3}\right)=\{\varnothing\}, I S\left(L_{4}\right)=\left\{L_{1}\right\}, \\
& I S\left(L_{5}\right)=\left\{L_{1}, L_{2}\right\} .
\end{aligned}
$$

Assuming $f(i)=1$, by Def.(8), link $L 3$ will be starved because $L_{2}$ has no link in its independent set i.e. $G_{P}\left(L_{3}\right)=0.0$, It follows that two subgroup $\left\{L_{1}, L_{2}\right\}$ and $\left\{L_{3}, L_{4}\right\}$ occupy full capacity for the ideal case because two border links are out of each other's carrier sensing range. In each subgroup, the optimistic goodput of a link will be proportional to the number of links in its independent set, and the corresponding expressions for goodput are:

$$
\begin{aligned}
& G_{O}\left(L_{1}\right)=\frac{\left|I S\left(L_{1}\right)\right|}{\left|I S\left(L_{1}\right)\right|+\left|I S\left(L_{2}\right)\right|+\left|I S\left(L_{3}\right)\right|}, \\
& G_{O}\left(L_{2}\right)=\frac{\left|I S\left(L_{2}\right)\right|}{\left|I S\left(L_{1}\right)\right|+\left|I S\left(L_{2}\right)\right|+\left|I S\left(L_{3}\right)\right|}, \\
& G_{O}\left(L_{1}\right)+G_{O}\left(L_{2}\right)+G_{P}\left(L_{3}\right)=1 .
\end{aligned}
$$

Similary, for the remaining subgroup:

$$
\begin{aligned}
& G_{O}\left(L_{4}\right)=\frac{\left|I S\left(L_{4}\right)\right|}{\left|I S\left(L_{3}\right)\right|+\left|I S\left(L_{4}\right)\right|+\left|I S\left(L_{5}\right)\right|}, \\
& G_{O}\left(L_{5}\right)=\frac{\left|I S\left(L_{4}\right)\right|}{\left|I S\left(L_{3}\right)\right|+\left|I S\left(L_{4}\right)\right|+\left|I S\left(L_{5}\right)\right|}, \\
& G_{O}\left(L_{4}\right)+G_{O}\left(L_{5}\right)+G_{P}\left(L_{3}\right)=1 .
\end{aligned}
$$

and this yields the solution we call the optimistic goodput.

By Def. 7. $\gamma^{G M C}=\left\{L_{1}, L_{5}\right\}$ and $\left|\gamma^{G M C}\right|=2$. By Def. 9 , the optimistic goodput of starving link $L_{3}$ is:

$$
G_{O}\left(L_{3}\right)=\alpha \times \frac{\left|\gamma^{G M C}\right|}{N}=0.08 \text { where } \alpha \text { is selected as } 0.2 \text {. }
$$

Since the starving link achieves non-zero goodput, the pessimistic goodput of other links need to consider it. For left border link $L_{1}$, its pessimistic goodput follows $G_{P}\left(L_{1}\right)+G_{P}\left(L_{2}\right)+G_{P}\left(L_{3}\right)=1$ and is: 


$$
G_{P}\left(L_{1}\right)=\frac{\left|I S\left(L_{1}\right)\right|}{\left|I S\left(L_{1}\right)\right|+\left|I S\left(L_{2}\right)\right|+\left|I S\left(L_{3}\right)\right|} \times\left(1-G_{O}\left(L_{3}\right)\right)
$$

For link $L_{2}, \gamma\left(L_{2}\right)=\left\{L_{1}, L_{3}, L_{4}\right\}$. We need to calculate the pessimistic goodput of link $L 2$ with consideration of the effect of link $L_{4}$. $G_{P}\left(L_{1}\right)+$ $G_{P}\left(L_{2}\right)+G_{P}\left(L_{3}\right)+G_{P}\left(L_{4}\right)=1$.

$$
G_{P}\left(L_{2}\right)=\frac{\left|I S\left(L_{1}\right)\right|}{\left|I S\left(L_{1}\right)\right|+\left|I S\left(L_{2}\right)\right|+\left|I S\left(L_{3}\right)\right|+\left|I S\left(L_{4}\right)\right|} \times\left(1-G_{O}\left(L_{3}\right)\right) .
$$

With the same reasoning, the pessimistic goodput of links $L_{4}$ and $L_{5}$ are:

$$
G_{P}\left(L_{5}\right)=\frac{\left|I S\left(L_{5}\right)\right|}{\left|I S\left(L_{5}\right)\right|+\left|I S\left(L_{4}\right)\right|+\left|I S\left(L_{3}\right)\right|} \times\left(1-G_{O}\left(L_{3}\right)\right) .
$$

For link $L_{4}, \gamma\left(L_{5}\right)=\left\{L_{2}, L_{3}, L_{5}\right\}$. We need to calculate the pessimistic goodput of link $L_{2}$ with consideration of the effect of link $L_{4}$.

$$
G_{P}\left(L_{4}\right)=\frac{\left|I S\left(L_{4}\right)\right|}{\left|I S\left(L_{5}\right)\right|+\left|I S\left(L_{4}\right)\right|+\left|I S\left(L_{3}\right)\right|+\left|I S\left(L_{2}\right)\right|} \times\left(1-G_{O}\left(L_{3}\right)\right) .
$$

By induction on $N$, we define the pessimistic and optimistic estimate of the goodput $G_{P}(i)$ and $G_{O}(i)$ for a given link for saturated traffic demand in ineffective carrier sensing scenario in Def.8 and Def. 9

\section{B.3 Goodput For Effective Carrier Sensing with Unsaturated Traffic Assumption}

Proof. Let $N$ be the number of links in a given IEEE 802.11 WBN and $f(i)$ the normalised traffic demand of a given link $i$ with respect to the maximum net capacity. 
For $N=1$, then there is one link $L_{1}$ in the IEEE $802.11 \mathrm{WBN}$. Since the normalised traffic demand of this link $f\left(L_{1}\right) \leq 1$, then $L_{1}$ achieves the desired goodput:

$$
G\left(L_{1}\right)=f(i) .
$$

For $N=2$, there are two links $L_{1}$ and $L_{2}$ in the IEEE $802.11 \mathrm{WBN}$. If the sum of the normalised traffic demands of these two links exceeds the capacity $f\left(L_{1}\right)+f\left(L_{2}\right)>1$, then these two links have to share the capacity and achieve the goodput as :

$$
G\left(L_{1}\right)=G\left(L_{2}\right)=\frac{1}{N}=0.5 .
$$

Therefore, $G\left(L_{i}\right)=\frac{1}{N}, \forall i \in N$.

If the sum of the traffic demands of these two links does not exceed the capacity $f\left(L_{1}\right)+f\left(L_{2}\right) \leq 1$, then these two links achieve the desired goodput:

$$
G\left(L_{1}\right)=G\left(L_{2}\right)=f(i) \text { and } G\left(L_{1}\right)+G\left(L_{2}\right) \leq 1 .
$$

Therefore, $G\left(L_{i}\right)=f(i), \forall i \in N$.

For $N=k$, there are $k$ links $L_{1}, \ldots$ and $L_{k}$ in the IEEE $802.11 \mathrm{WBN}$. If the sum of the traffic demands of these $k$ links exceeds the capacity $\sum_{j \in E} f(j)=k>1$, then these $k$ links have to share the capacity and achieve the goodput as:

$$
G\left(L_{1}\right)=\ldots=G\left(L_{k}\right)=\frac{1}{k}=\frac{1}{N} \text { and } G\left(L_{1}\right)+\ldots+G\left(L_{k}\right)=1 .
$$

Therefore, $G\left(L_{i}\right)=\frac{1}{N^{\prime}}, \forall i \in N$.

If the sum of the traffic demands of these $k$ links does not exceed the capacity $\sum_{j \in E} f(j)=k \leq 1$, then these $k$ links achieve desired goodput as:

$$
G\left(L_{1}\right)=\ldots=G\left(L_{k}\right)=f(i) \text { and } G\left(L_{1}\right)+\ldots+G\left(L_{k}\right) \leq 1 \text {. }
$$

Therefore, $G\left(L_{i}\right)=f(i), \forall i \in N$.

By induction on $N$, the goodput for a given link for unsaturated traffic demand in effective carrier sensing scenario in Def. 10 . 


\section{Appendix C}

\section{Additional Validation of the Proposed Goodput Model Using IEEE 802.11a $48 \mathrm{Mbps}$}

The chapter discusses the validation of the proposed goodput distribution model defined in Chapter 3 on page 41 by using a different data rate of IEEE $802.11 \mathrm{a} 48 \mathrm{Mbps}$. The reason to choose IEEE $802.11 \mathrm{a} 48 \mathrm{Mbps}$ is to validate the accuracy of the proposed goodput model in a higher data rate.

The next sections are the simulation results with two-ray and two-ray shadowing propagation models.

\section{C.1 Simulation validation of the goodput model with two-ray propagation model using IEEE 802.11a 48 Mbps}

This section validates the accuracy of the proposed goodput model through simulation with the two-ray ground model [66].

The configuration parameters are listed in Tables C.1. Similar to the validation in Section 3.2 on page 59, (i) all nodes are configured with iden- 
tical parameters and choose constant bit rate (CBR) unicast transmission as the application with saturated and unsaturated traffic demands(see Table C.1); (ii) all nodes are configured with one radio interface and the same channel. Node placement is based on a grid topology (see Figure 3.3 on page 60); (iii) transmitter-receiver separation is chosen as $20 \mathrm{~m}$ to guarantee collision-free transmissions (based on the findings from [156]). Carrier sensing range $R_{C S}$ is calculated as $435 \mathrm{~m}$ with the configuration in Table C.1.

Table C.1: Simulation configuration parameters

\begin{tabular}{l|l}
\hline Parameter Name & Value \\
\hline Transmission Power & $16 \mathrm{dBm}$ \\
\hline Receiver Sensitivity & $-69 \mathrm{dBm}$ \\
\hline Path Loss Model & Two-Ray \\
\hline Shadowing and Fading Model & None \\
\hline Physical Layer & IEEE $802.11 \mathrm{a}$ \\
\hline Data Rate & $48 \mathrm{Mbps}$ \\
\hline MAC Layer & PCS \\
\hline Routing & Static Routing \\
\hline Transportation Layer & UDP \\
\hline Packet Size & 1500 Bytes \\
\hline Inter-packet Interval for Saturated Traffic & $0.25 \mathrm{~ms}$ \\
\hline Inter-packet Interval for Unsaturated Traffic & $0.25-4.26 \mathrm{~ms}$ \\
\hline
\end{tabular}

The results shown for the average goodput in this section are calculated from 100 randomly seeded simulation runs. All averages shown are reported with confidence interval of $95 \%$ given by the range of $[0.013,36.56]$ kbps under the assumption that the averages are normally distributed.

The next sections categorise the simulation results by using two-ray propagation model are into two parts, saturated and unsaturated traffic demands. 


\section{C.1.1 Saturated Traffic Demand}

Due to space, two simulation results are listed for effective and ineffective carrier sensing scenarios. For the effective carrier sensing scenario, the topology in Figure 3.3 on page 60 is selected with the border distance $D$ as $200 \mathrm{~m}$, where all nodes are within each other's $R_{c s}$. For ineffective carrier sensing scenarios, the topology in Figure 3.3 is selected with the border distance $D$ as $600 \mathrm{~m}$, where two border links are out of each other's carrier sensing range.

Table C.2: Goodput of five links in a $200 \times 20 \mathrm{~m}^{2}$ topology with two-ray propagation model

\begin{tabular}{cccc}
\hline Link $i$ & $G_{S}(i)$ & $G_{P}(i) / G_{O}(i)$ & $E R$ \\
\hline 1 & 0.161 & 0.200 & - \\
2 & $0.213^{*}$ & 0.200 & 0.059 \\
3 & $0.248^{*}$ & 0.200 & 0.195 \\
4 & $0.216^{*}$ & 0.200 & 0.073 \\
5 & 0.162 & 0.200 & - \\
\hline
\end{tabular}

Table C.3: Goodput of six links in a $600 \times 20 \mathrm{~m}^{2}$ topology with two-ray propagation model

\begin{tabular}{ccccc}
\hline Link $i$ & $G_{S}(i)$ & $G_{P}(i)$ & $G_{O}(i)$ & $E R$ \\
\hline 1 & 0.614 & 0.552 & 0.667 & - \\
2 & 0.328 & 0.250 & 0.333 & - \\
3 & 0.034 & 0.000 & 0.067 & - \\
4 & 0.034 & 0.000 & 0.067 & - \\
5 & 0.328 & 0.250 & 0.333 & - \\
6 & 0.616 & 0.552 & 0.667 & - \\
\hline
\end{tabular}

In effective carrier sensing scenarios with saturated traffic demand, the results in Table C.2 show that different links achieve different goodput. 
We mark the simulation results that slightly exceed the prediction from the new model with $\left(^{*}\right)$. In the new goodput model, we assume that all links share the channel capacity, which matches with the expectation from disk-graph model. However, in practice there is a small variation in goodput among all the links. In this case, middle links achieve higher goodput than the border links. It implies that even in effective carrier sensing scenarios, the carrier sensing scheme cannot control the media access among neighbouring links fairly. The overall different ratio is below $20 \%$.

For the ineffective scenario, the topology is chosen with a fixed border distance, $D=600 \mathrm{~m}$ to ensure that the two border links are always out of each other's $R_{c s}$ and border effect should occur. Table C.3 displays the comparison between the results from simulations and those from the new goodput model. The grey cells in these two tables denote the links identified as starving links.

The data in Table C.3 shows the new model predicts starving links correctly. For the non-starving links, all the simulation mean values fall between the range of the predictions from the new model. In ineffective carrier sensing scenarios with saturated traffic demands, the proposed goodput model provides accurate prediction of starvation and goodput.

\section{C.1.2 Unsaturated Traffic Demand}

To validate the new model with unsaturated traffic demand, three unsaturated traffic demands are selected by using different inter-packet intervals. The traffic demands $f(i)$ at the application layer can be normalised with respect of the maximum net bandwidth as $0.8,0.6,0.4$, and 0.1 with the above inter-packet intervals and the configuration parameters in Table C.1.

The results of the effective carrier sensing scenario $(D=200 \mathrm{~m})$ are list the results in Tables C.4 and C.5. The function $f(i)$ refers to the traffic demand while goodput $G_{S}(i)$ and $G_{P}(i) / G_{O}(i)$ denote the simulation goodput mean values and the predicted goodput from the new model.

The results show the same goodput pattern as that in saturated traffic 
Table C.4: Goodput of five links in a $200 \times 20 \mathrm{~m}^{2}$ topology with two-ray propagation model

\begin{tabular}{c|cccc|cccc}
\hline Link $i$ & $f(i)$ & $G_{S}(i)$ & $G_{P}(i) / G_{O}(i)$ & $E R$ & $f(i)$ & $G_{S}(i)$ & $G_{P}(i) / G_{O}(i)$ & $E R$ \\
\hline 1 & 0.8 & 0.161 & 0.200 & - & 0.6 & 0.161 & 0.200 & - \\
2 & 0.8 & $0.214^{*}$ & 0.200 & 0.065 & 0.6 & $0.214^{*}$ & 0.200 & 0.065 \\
3 & 0.8 & $0.249^{*}$ & 0.200 & 0.197 & 0.6 & $0.251^{*}$ & 0.200 & 0.203 \\
4 & 0.8 & $0.214^{*}$ & 0.200 & 0.065 & 0.6 & $0.214^{*}$ & 0.200 & 0.065 \\
5 & 0.8 & 0.162 & 0.200 & - & 0.6 & 0.161 & 0.200 & - \\
\hline
\end{tabular}

Table C.5: Goodput of five links in a $200 \times 20 \mathrm{~m}^{2}$ topology with two-ray propagation model-continued

\begin{tabular}{c|cccc|cccc}
\hline Link $i$ & $f(i)$ & $G_{S}(i)$ & $G_{P}(i) / G_{O}(i)$ & $E R$ & $f(i)$ & $G_{S}(i)$ & $G_{P}(i) / G_{O}(i)$ & $E R$ \\
\hline 1 & 0.4 & 0.162 & 0.200 & - & 0.1 & 0.100 & 0.100 & - \\
2 & 0.4 & $0.214^{*}$ & 0.200 & 0.065 & 0.1 & 0.100 & 0.100 & - \\
3 & 0.4 & $0.253^{*}$ & 0.200 & 0.209 & 0.1 & 0.100 & 0.100 & - \\
4 & 0.4 & $0.212^{*}$ & 0.200 & 0.057 & 0.1 & 0.100 & 0.100 & - \\
5 & 0.4 & 0.160 & 0.200 & - & 0.1 & 0.100 & 0.100 & - \\
\hline
\end{tabular}

demand. Except the case $f(i)=0.1$ that all links achieve the same goodput, for other traffic demands, small variation exists among all the links. Along the same lines of explanation in Section 3.2.1 on page 61. in effective carrier sensing scenarios, the randomised binary exponential back-off scheme used in IEEE 802.11 carrier sensing may result in a slight difference among the goodput of neighbouring links rather than achieving extreme equal goodput for each link [10]. The overall error is below $21 \%$.

Tables C.6 and C.7 list the results with unsaturated traffic demands in the ineffective carrier sensing scenarios where $D$ is selected as $600 \mathrm{~m}$ and $d$ as $120 \mathrm{~m}$. Results in Tables C.6 and C.7 show that most of the 
Table C.6: Goodput of six links in a $600 \times 20 \mathrm{~m}^{2}$ topology with two-ray propagation model

\begin{tabular}{c|cccc|cccc}
\hline & \multicolumn{5}{|c|}{$f(i)=0.8$} & \multicolumn{4}{c}{$f(i)=0.6$} \\
Link $i$ & $G_{S}(i)$ & $G_{O}(i)$ & $G_{P}(i)$ & $E R$ & $G_{S}(i)$ & $G_{O}(i)$ & $G_{P}(i)$ & $E R$ \\
\hline 1 & 0.641 & 0.552 & 0.667 & - & 0.5997 & 0.486 & 0.600 & - \\
2 & 0.328 & 0.250 & 0.333 & - & $0.339^{*}$ & 0.250 & 0.333 & 0.018 \\
3 & 0.016 & 0.000 & 0.067 & - & 0.026 & 0.000 & 0.067 & - \\
4 & 0.016 & 0.000 & 0.067 & - & 0.027 & 0.000 & 0.067 & - \\
5 & 0.327 & 0.250 & 0.333 & - & $0.339^{*}$ & 0.250 & 0.333 & 0.018 \\
6 & 0.641 & 0.552 & 0.667 & - & 0.5997 & 0.486 & 0.600 & - \\
\hline
\end{tabular}

Table C.7: Goodput of six links in a $600 \times 20 \mathrm{~m}^{2}$ topology with two-ray propagation model-continued

\begin{tabular}{c|cccc|cccc}
\hline & \multicolumn{5}{|c|}{$f(i)=0.4$} & \multicolumn{4}{c}{$f(i)=0.1$} \\
Link $i$ & $G_{S}(i)$ & $G_{P}(i)$ & $G_{O}(i)$ & $E R$ & $G_{S}(i)$ & $G_{P}(i)$ & $G_{O}(i)$ & $E R$ \\
\hline 1 & 0.400 & 0.360 & 0.400 & - & 0.1 & 0.100 & 0.100 & - \\
2 & 0.356 & 0.300 & 0.400 & - & 0.1 & 0.100 & 0.100 & - \\
3 & 0.094 & 0.090 & 0.170 & - & 0.1 & 0.100 & 0.100 & - \\
4 & 0.093 & 0.090 & 0.170 & - & 0.1 & 0.100 & 0.100 & - \\
5 & 0.355 & 0.300 & 0.400 & - & 0.1 & 0.100 & 0.100 & - \\
6 & 0.400 & 0.360 & 0.400 & - & 0.1 & 0.100 & 0.100 & - \\
\hline
\end{tabular}

simulation mean values fall between the prediction range, proving the proposed model predicts starvation and goodput distribution accurately. When $f(i)=0.6$, in links 2 and 5, a small difference between the simulation results and the prediction from the new model. The overall error is below $2 \%$. 


\section{C.2 Simulation validation with two-ray shadow- ing propagation model using IEEE 802.11a $48 \mathrm{Mbps}$}

This section uses the two-ray shadowing propagation model [66] in the simulations to demonstrate the generalizability of the new model. The simulation results are presented in two parts: saturated and unsaturated traffic demands.

For two-ray ground shadowing model, three shadowing attenuations $\delta$ are selected as 2,4 , and 6 . The greater number of shadowing attenuation, the more obstacles exist in the propagation path. The higher the value of shadowing attenuation $\delta$ is, the greater the number of obstructions along the propagation path is assumed to be. The carrier sensing range $R_{C S}$ for $\delta$ as 2,4 ,and 6 is $340 \mathrm{~m}, 270 \mathrm{~m}$, and $215 \mathrm{~m}$ respectively.

\section{C.2.1 Saturated Traffic Demand}

Table C.8: Goodput of five links in a $200 \times 50 \mathrm{~m}^{2}$ topology with two-ray shadowing propagation model

\begin{tabular}{c|c|cc|cc|cc}
\hline \multirow{2}{*}{ Link $i$} & \multicolumn{2}{|c|}{ Propagation Model } & \multicolumn{2}{|c|}{ Shadowing $(\delta=2)$} & \multicolumn{2}{|c}{ Shadowing $(\delta=4)$} & \multicolumn{2}{|c}{ Shadowing $(\delta=6)$} \\
$G_{P}(i) / G_{O}(i)$ & $G_{S}(i)$ & $E R$ & $G_{S}(i)$ & $E R$ & $G_{S}(i)$ & $E R$ \\
\hline 1 & 0.200 & 0.157 & - & 0.156 & - & 0.166 & - \\
2 & 0.200 & $0.223^{*}$ & 0.103 & $0.226^{*}$ & 0.115 & $0.219^{*}$ & 0.087 \\
3 & 0.200 & $0.238^{*}$ & 0.160 & $0.236^{*}$ & 0.153 & $0.230^{*}$ & 0.130 \\
4 & 0.200 & $0.226^{*}$ & 0.115 & $0.224^{*}$ & 0.103 & $0.219^{*}$ & 0.087 \\
5 & 0.200 & 0.156 & - & 0.116 & - & 0.166 & - \\
\hline
\end{tabular}

For saturated traffic demand, three topologies are selected representing effective and ineffective carrier sensing scenarios. Table C.8 shows the results of effective carrier sensing scenario $(D=200 \mathrm{~m})$. The results show 
Table C.9: Goodput of six links in a $500 \times 50 \mathrm{~m}^{2}$ topology with two-ray shadowing propagation model

\begin{tabular}{c|cccc|ccccc|cccc}
\hline & \multicolumn{3}{|c|}{ Shadowing $(\delta=2)$} & \multicolumn{5}{c|}{ Shadowing $(\delta=4)$} & \multicolumn{5}{c}{ Shadowing $(\delta=6)$} \\
Link $i$ & $G_{S}(i)$ & $G_{P}(i)$ & $G_{O}(i)$ & $E R$ & $G_{S}(i)$ & $G_{P}(i)$ & $G_{O}(i)$ & $E R$ & $G_{S}(i)$ & $G_{P}(i)$ & $G_{O}(i)$ & $E R$ \\
\hline 1 & 0.616 & 0.552 & 0.667 & - & 0.647 & 0.552 & 0.667 & - & 0.515 & 0.450 & 0.500 & - \\
2 & 0.328 & 0.250 & 0.333 & - & 0.330 & 0.250 & 0.333 & - & 0.314 & 0.286 & 0.333 & - \\
3 & 0.034 & 0.000 & 0.067 & - & 0.012 & 0.000 & 0.067 & - & $0.171^{*}$ & 0.111 & 0.167 & 0.023 \\
4 & 0.034 & 0.000 & 0.067 & - & 0.012 & 0.000 & 0.067 & - & $0.170^{*}$ & 0.111 & 0.167 & 0.018 \\
5 & 0.328 & 0.250 & 0.333 & - & 0.329 & 0.250 & 0.333 & - & 0.314 & 0.286 & 0.333 & - \\
6 & 0.616 & 0.552 & 0.667 & - & 0.648 & 0.552 & 0.667 & - & 0.515 & 0.450 & 0.500 & - \\
\hline
\end{tabular}

that adding a shadowing factor in the propagation model does not change the goodput pattern observed in two-ray propagation model. The links in the effective carrier sensing scenario do not share the channel capacity equally. Middle links achieve higher goodput than the border links. But in Table C.8, the overall error is below $16 \%$, which is lower than that in the two-ray propagation model. It could be the interaction between border links and middle links decreases with shadowing effect.

For ineffective scenarios, the topology is chosen with the fixed border distance, $500 \mathrm{~m}$ and $d$ as $100 \mathrm{~m}$. Tables C.9 shows that the proposed model predicts starvation accurately and few errors happen in the scenarios where shadowing attenuation is configured as 6 . The overall error is below $2.5 \%$.

\section{C.2.2 Unsaturated Traffic Demand}

For unsaturated traffic demand, only the results with shadowing attenuation $\delta$ as 4 is listed due to space. Shadowing attenuation $\delta=4$ is the default value in QualNet 5.2 that refers to the common indoor environments with four walls.

Tables C.10 to C.11 list the results from effective carrier sensing scenario $(D=200 \mathrm{~m})$. When the sum of traffic demands of all links exceed 
Table C.10: Goodput of five links in a $200 \times 20 \mathrm{~m}^{2}$ topology with two-ray shadowing propagation model $(\delta=4)$

\begin{tabular}{c|cccc|cccc}
\hline Link $i$ & $f(i)$ & $G_{S}(i)$ & $G_{P}(i) / G_{O}(i)$ & $E R$ & $f(i)$ & $G_{S}(i)$ & $G_{P}(i) / G_{O}(i)$ & $E R$ \\
\hline 1 & 0.8 & 0.154 & 0.200 & - & 0.6 & 0.156 & 0.200 & - \\
2 & 0.8 & $0.225^{*}$ & 0.200 & 0.111 & 0.6 & $0.224^{*}$ & 0.200 & 0.107 \\
3 & 0.8 & $0.239^{*}$ & 0.200 & 0.163 & 0.6 & $0.237^{*}$ & 0.200 & 0.156 \\
4 & 0.8 & $0.224^{*}$ & 0.200 & 0.107 & 0.6 & $0.228^{*}$ & 0.200 & 0.123 \\
5 & 0.8 & 0.157 & 0.200 & - & 0.6 & 0.156 & 0.200 & - \\
\hline
\end{tabular}

Table C.11: Goodput of five links in a $200 \times 20 \mathrm{~m}^{2}$ topology with two-ray shadowing propagation model $(\delta=4)$-continued

\begin{tabular}{c|cccc|cccc}
\hline Link $i$ & $f(i)$ & $G_{S}(i)$ & $G_{P}(i) / G_{O}(i)$ & $E R$ & $f(i)$ & $G_{S}(i)$ & $G_{P}(i) / G_{O}(i)$ & $E R$ \\
\hline 1 & 0.4 & 0.154 & 0.200 & - & 0.1 & 0.100 & 0.100 & - \\
2 & 0.4 & $0.226^{*}$ & 0.200 & 0.115 & 0.1 & 0.100 & 0.100 & - \\
3 & 0.4 & $0.239^{*}$ & 0.200 & 0.163 & 0.1 & 0.100 & 0.100 & - \\
4 & 0.4 & $0.226^{*}$ & 0.200 & 0.115 & 0.1 & 0.100 & 0.100 & - \\
5 & 0.4 & 0.155 & 0.200 & - & 0.1 & 0.100 & 0.100 & - \\
\hline
\end{tabular}

Table C.12: Goodput of six links in a $425 \times 20 \mathrm{~m}^{2}$ topology with two-ray shadowing propagation model $(\delta=4)$

\begin{tabular}{c|cccc|cccc}
\hline & \multicolumn{5}{|c|}{$f(i)=0.8$} & \multicolumn{4}{c}{$f(i)=0.6$} \\
Link $i$ & $G_{S}(i)$ & $G_{O}(i)$ & $G_{P}(i)$ & $E R$ & $G_{S}(i)$ & $G_{O}(i)$ & $G_{P}(i)$ & $E R$ \\
\hline 1 & 0.648 & 0.552 & 0.667 & - & 0.600 & 0.486 & 0.600 & - \\
2 & 0.329 & 0.250 & 0.333 & - & $0.344^{*}$ & 0.250 & 0.333 & 0.032 \\
3 & 0.012 & 0.000 & 0.067 & - & 0.023 & 0.000 & 0.067 & - \\
4 & 0.012 & 0.000 & 0.067 & - & 0.023 & 0.000 & 0.067 & - \\
5 & 0.330 & 0.250 & 0.333 & - & $0.344^{*}$ & 0.250 & 0.333 & 0.032 \\
6 & 0.647 & 0.552 & 0.667 & - & 0.600 & 0.486 & 0.600 & - \\
\hline
\end{tabular}


Table C.13: Goodput of six links in a $425 \times 20 \mathrm{~m}^{2}$ topology with two-ray shadowing propagation model $(\delta=4)$-continued

\begin{tabular}{c|cccc|cccc}
\hline & \multicolumn{5}{|c|}{$f(i)=0.4$} & \multicolumn{4}{c}{$f(i)=0.1$} \\
Link $i$ & $G_{S}(i)$ & $G_{P}(i)$ & $G_{O}(i)$ & $E R$ & $G_{S}(i)$ & $G_{P}(i)$ & $G_{O}(i)$ & $E R$ \\
\hline 1 & 0.400 & 0.360 & 0.400 & - & 0.1 & 0.100 & 0.100 & - \\
2 & 0.366 & 0.300 & 0.400 & - & 0.1 & 0.100 & 0.100 & - \\
3 & $0.084^{*}$ & 0.090 & 0.17 & 0.071 & 0.1 & 0.100 & 0.100 & - \\
4 & $0.084^{*}$ & 0.090 & 0.17 & 0.071 & 0.1 & 0.100 & 0.100 & - \\
5 & 0.366 & 0.300 & 0.400 & - & 0.1 & 0.100 & 0.100 & - \\
6 & 0.400 & 0.360 & 0.400 & - & 0.1 & 0.100 & 0.100 & - \\
\hline
\end{tabular}

the channel capacity, the difference of goodput between middle links and border links exists. The overall error is below $20 \%$.

For the ineffective carrier sensing scenarios $(D=425 \mathrm{~m}$ and $d=85 \mathrm{~m})$, the proposed model can predict starving links correctly in Tables C.12 to C.13. Few simulation results of non-starving links are out of the range of the prediction from the new model. The overall error is below $7.5 \%$. 


\section{Appendix D}

\section{Investigation Flow Starvation with Interference Models and Channel Assignment in IEEE 802.11 WBNs}

This chapter investigates fairness with three different interference models used in channel assignment (CA) algorithms. By analysing fairness with different interference models, we discuss the relationship between flow starvation and interference models. The discussion aims to identify the shortcomings of these interference models to guide CA algorithms to prevent flow starvation. The findings in this chapter inform the design of the proposed interference model in Chapter 4 .

Here, a clique-based channel assignment INSTC algorithm [140] is selected and defined in Section 4.2.2.4 on page 95. The INSTC algorithm integrates three interference set selections based on three common interference models defined in Section 4.2.2 on page 92. The inputs of the INSTC algorithm and simulation configurations are the same as those in Section 4.2.3.1 on page 97 .

Simulation results shown in this section are averages from 100 ran- 
domly seeded simulation runs. All averages shown are reported with confidence interval of $95 \%$ with the range of $[5.2,15.66]$ kbps under the assumption that the averages are normally distributed.

The next sections will investigate how different interference set selections influence network fairness from the view of different fairness measures, Jain's index [33], starvation link ratio, and highest-to-lowest goodput ratio [131] defined in Section 4.2.3.1 on page 97.

\section{D.1 Comparison of Jain's Index}

Figure D.1 show the results of Jain's index from the clique-based CA algorithm using interference set selections $\gamma_{A}, \gamma_{B}$, and $\gamma_{C}$ in grid topologies. In Figure D.1, the $X$ axis denotes the border distance $D$ from $200 \mathrm{~m}$ to $800 \mathrm{~m}$ while the $Y$ axis refers to the achieved Jain's index of the channel allocations from the clique-based CA algorithm using different interference set selections.

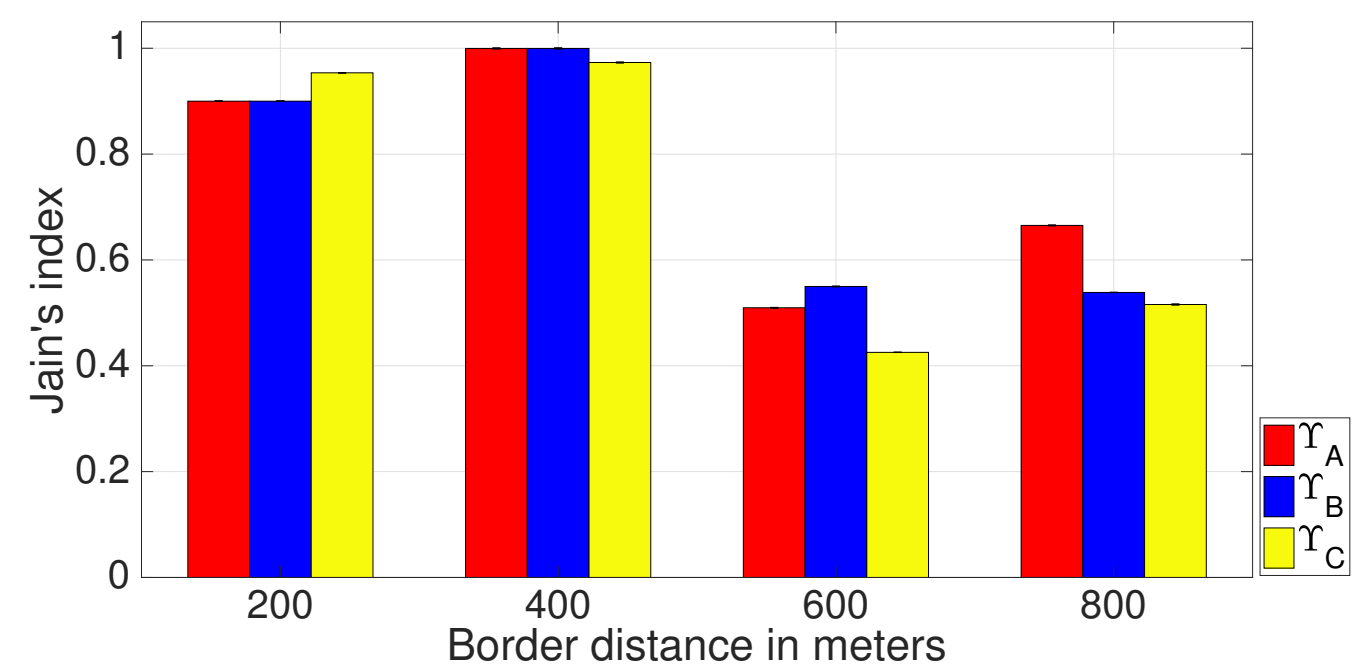

Figure D.1: The comparison of Jain's index with different interference sets in grid topologies 
In the figure, when border distances $D$ is less than $R_{C S}$ ( i.e. $200 \mathrm{~m}$ and $400 \mathrm{~m}$, all nodes are within each other's carrier sensing range, so called effective carrier sensing scenarios), the Jain's indexes of the clique-based CA algorithm using three interference set selections $\gamma_{A}, \gamma_{B}$, and $\gamma_{C}$ are similar that are between 0.9 and 1.0 .

When border distances $D$ is greater than $R_{c s}$ (i.e. $600 \mathrm{~m}$ and $800 \mathrm{~m}$, not all nodes are within each other's carrier sensing range, so called ineffective carrier sensing scenarios), the Jain's indexes from the clique-based CA algorithm using three interference set selections $\gamma_{A}, \gamma_{B}$, and $\gamma_{C}$ decrease to between 0.4 and 0.7 .

Overall, in effective carrier sensing scenarios, fairness index is satisfactory because carrier sensing mechanism manages the medium access among links well and border effect does exist. With the increase of border distance, the Jain's index of three interference set selections decreases significantly with the increase of border distance in grid topologies. In ineffective carrier sensing scenarios, exposed nodes, hidden nodes, and border effect bring more influences in fairness.

\section{D.2 Comparison of Starvation Link Ratio}

Figure D.2 shows the results of starvation link ratio from the clique-based CA algorithm using interference set selections $\gamma_{A}, \gamma_{B}$, and $\gamma_{C}$ in grid topologies. In Figure D.2, the $\mathrm{X}$ axis denotes the border distance while the $\mathrm{Y}$ axis refers to the starvation link ratio from the clique-based CA algorithm using different interference set selections.

In the figure, when border distance $D$ is less than $R_{c s}$ (i.e. $200 \mathrm{~m}$ and $400 \mathrm{~m}$, the effective carrier sensing scenarios), the starvation link ratios of three interference set selections $\gamma_{A}, \gamma_{B}$, and $\gamma_{C}$ are zero, which means flow starvation does not exist.

When border distance $D$ is greater than $R_{c S}$ (i.e. $600 \mathrm{~m}$ and $800 \mathrm{~m}$, the ineffective carrier sensing scenarios), the starvation link ratio from $\gamma_{A}, \gamma_{B}$, 


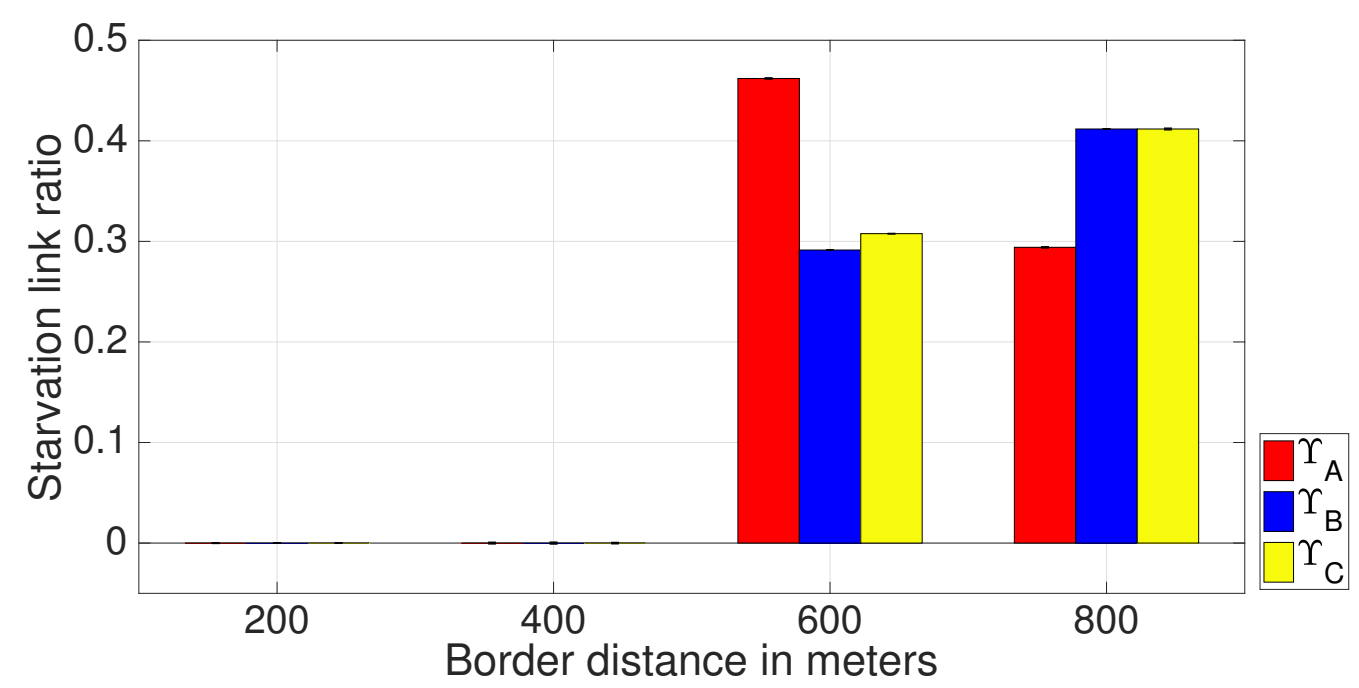

Figure D.2: Starvation link ratio with different interference sets in grid topologies

and $\gamma_{C}$ increases up to 0.45 . The trend of starvation link ratio is opposite to that of Jain's index. The higher starvation link ratio is, the lower Jain's index is. It implies flow starvation contributes toward poor fairness.

\section{D.3 Comparison of Highest-to-lowest Goodput Ratio}

Figure D.3 shows the results of highest-to-lowest goodput ratio from the clique-based CA algorithm using interference set selections $\gamma_{A}, \gamma_{B}$, and $\gamma_{C}$ in grid topologies. In Figure D.2, the $X$ axis denotes the border distance while the $\mathrm{Y}$ axis refers to the highest-to-lowest goodput ratio from the clique-based CA algorithm using different interference set selections.

In the figure, when the border distance $D$ is less than $R_{c S}$ (i.e. $200 \mathrm{~m}$ and $400 \mathrm{~m}$, the effective carrier sensing scenarios), the highest-to-lowest goodput ratios of three interference set selections $\gamma_{A}, \gamma_{B}$, and $\gamma_{C}$ are very small. 


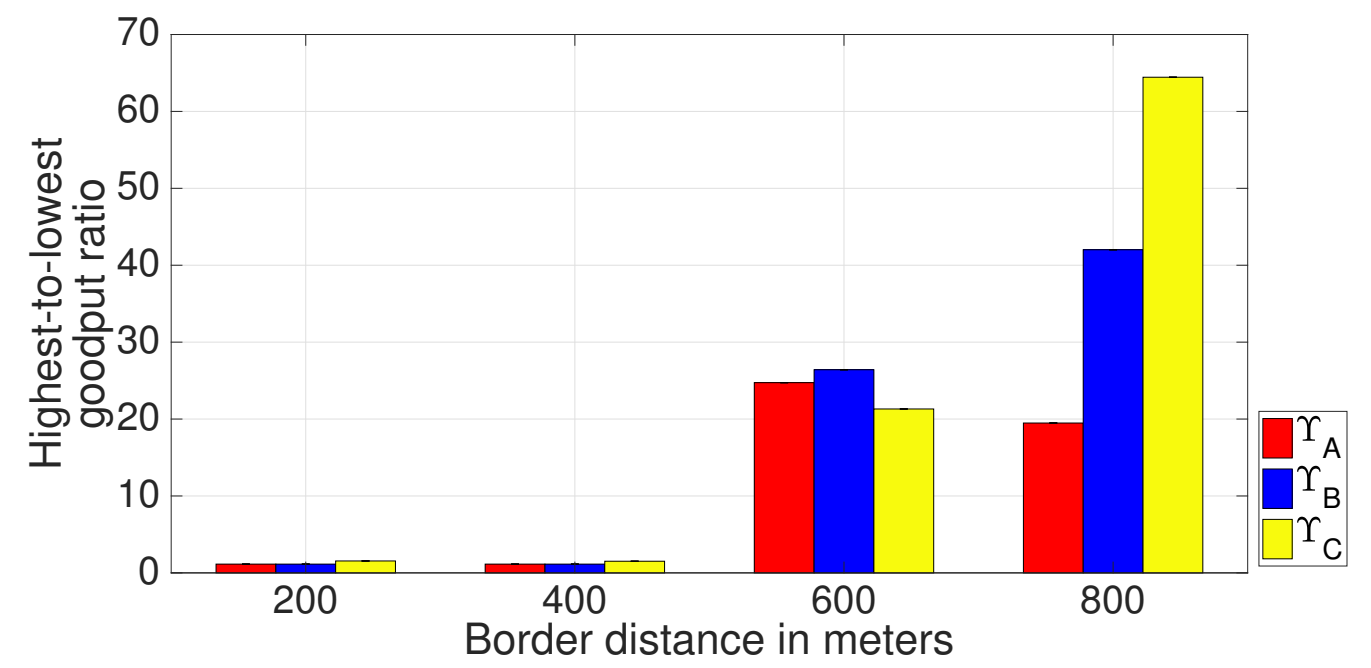

Figure D.3: Highest-to-lowest goodput ratio with different interference sets in grid topologies

When the border distance $D$ is greater than $R_{c s}$ (i.e. $600 \mathrm{~m}$ and $800 \mathrm{~m}$, the ineffective carrier sensing scenarios), the highest-to-lowest goodput ratio from $\gamma_{A}, \gamma_{B}$, and $\gamma_{C}$ increases up to over 60 . The high highest-tolowest goodput ratio in Figure D.3 shows a different trend compared with the Jain's index in Figure D.1. It matches with the expectation that high highest-to-lowest goodput ratio indicates a low Jain's index.

\section{D.4 Jain's Index vs. Starvation Link Ratio}

To explore the relation between Jain's index and starvation link ratio, we use a scatter plot in Figure D.4. In Figure D.4 the $\mathrm{X}$ axis denotes the Jain's index while the $\mathrm{Y}$ axis refers to the starvation link ratio.

In the figure, the Jain's index is not proportional to the starvation link ratio. Overall, the greater starvation link ratio, the smaller Jain's index. when starvation ratio is 0 , Jain's index is between 0.9 and 1.0. The points have starvation link ratios around 0.3 but their Jain's indexes are quite 
different. The points have Jain's index around 0.54 and their starvation link ratios are much different. It implies that Jain's index is not sensitive to flow starvation and evaluating fairness needs both metrics.

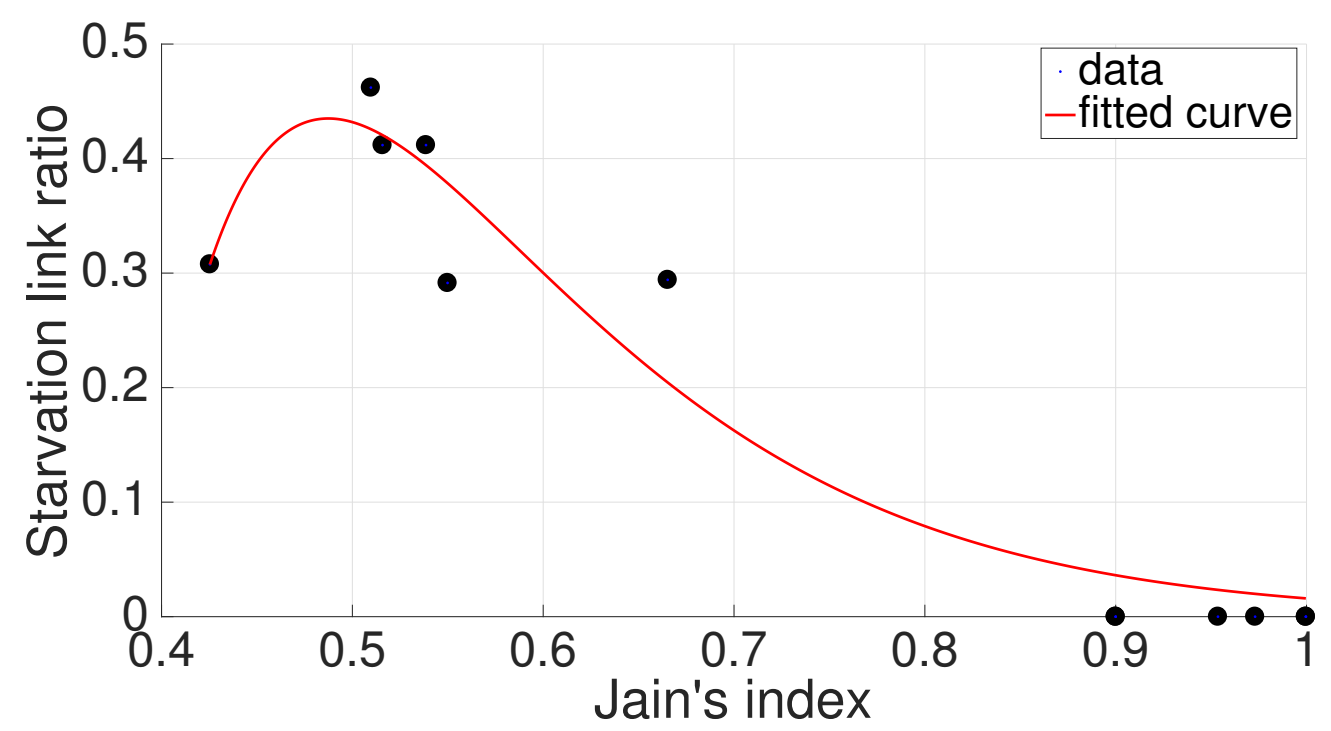

Figure D.4: Jain's index vs starvation ratio with different interference sets in grid topologies

\section{D.5 Discussing Flow Starvation with Interference Set Selections}

Based on the above analysis under saturated traffic assumption, we will discuss flow starvation with the three interference set selections used in the clique-based CA algorithm from two aspects. The first aspect is the fairness pattern in IEEE 802.11 WBNs and the second aspect is the root cause of unfairness (flow starvation) with respect to the interference set selections.

In terms of fairness, three interference set selections yield similar pat- 
terns in both effective and ineffective carrier sensing scenarios. In effective carrier sensing scenarios, all interference set selections yield high Jain's index, "zero" starvation link ratio, and low highest-to-lowest goodput ratio. Because flow starvation does not exit in effective carrier sensing scenarios, fairness is satisfactory. However, in ineffective carrier sensing scenarios, all interference set selections yield poor Jain's index, high starvation link ratio, and high highest-to-lowest goodput ratio compared with those in effective carrier sensing scenarios.

To analyse the root cause of unfairness with interference set selections, we need to look into flow starvation. In effective carrier sensing scenarios, flow starvation does exist and the border effect (we call it as global interference) between border links and middle links does exit. The interference exists locally among neighbouring links. Hence, the clique-based CA algorithm effectively manages the network fairness by using three interference set selections reflecting local interference among neighbouring links.

In ineffective carrier sensing scenarios, three interference set selections in the clique-based CA algorithm failed to prevent flow starvation that leads to poor fairness. It is because these interference set selections used in the CA algorithm only reflect local interference but not the global interference.

Next, we further explain why the existing interference set selections fail to prevent flow starvation and yield poor network fairness in ineffective carrier sensing scenario.

We use an example to explore the reason. Figures D.5 to D.8 show how the clique-based CA algorithm using interference set $\gamma_{A}$ allocates channels to links $L 1$ to $L 11$ in the $1000 \times 100 \mathrm{~m}^{2}$ topology (See Figure 4.5). Assume that carrier sensing range $R_{C S}$ is $710 \mathrm{~m}$. In Figure D.5, the CA algorithm starts from link $L 1$ and selects the least-used channel 1 in red within link L1's conflict set as none of the channel has been used before.

In Figure D.6, the CA algorithm checks link L2's interference set and 


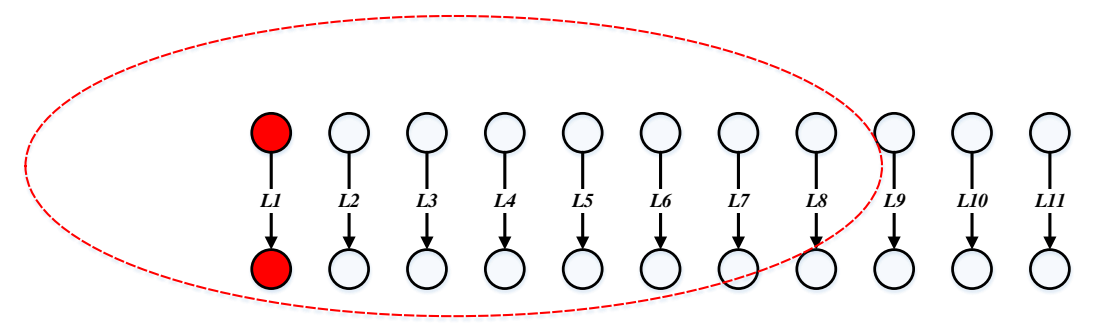

Figure D.5: Step 1: allocate a channel to link $L 1$

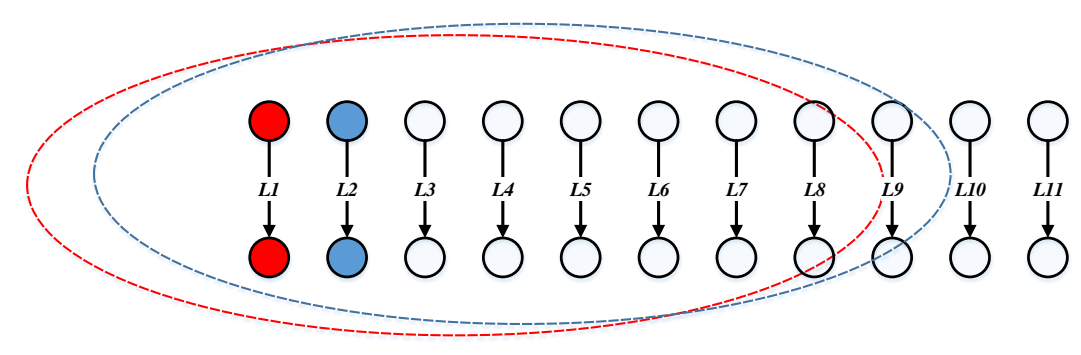

Figure D.6: Step 2: allocate a channel to link L2

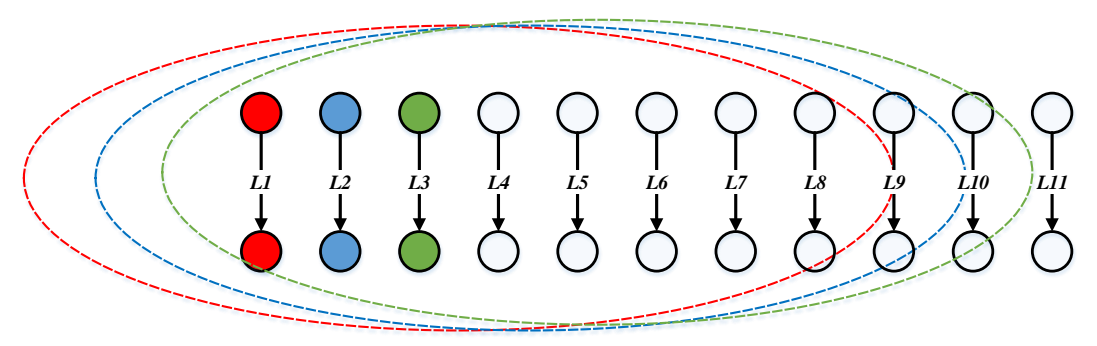

Figure D.7: Step 3: allocate a channel to link $L 3$

selects channel 2 in blue because channel 1 has been used. As same as links $L 1$ and $L 2$, link $L 3$ has been allocated with the least used channel 3 in green (see Figure D.7) In the end, all the links have been allocated with the least used channel within its interference set (see Figure D.8).

In Figure D.9, we only list the links allocated with channel 1 from Fig- 


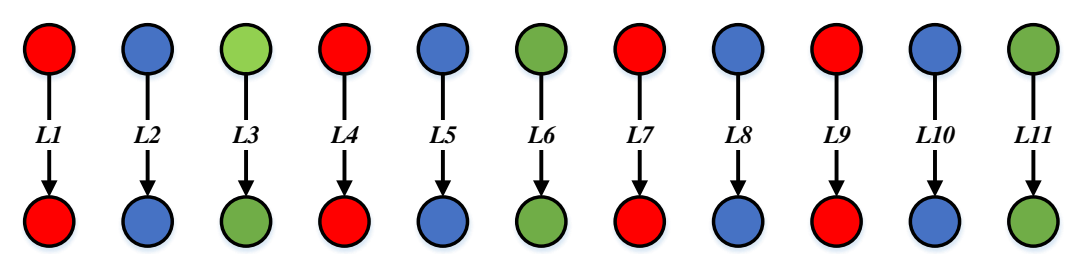

Figure D.8: Final step: Channels allocated to all links

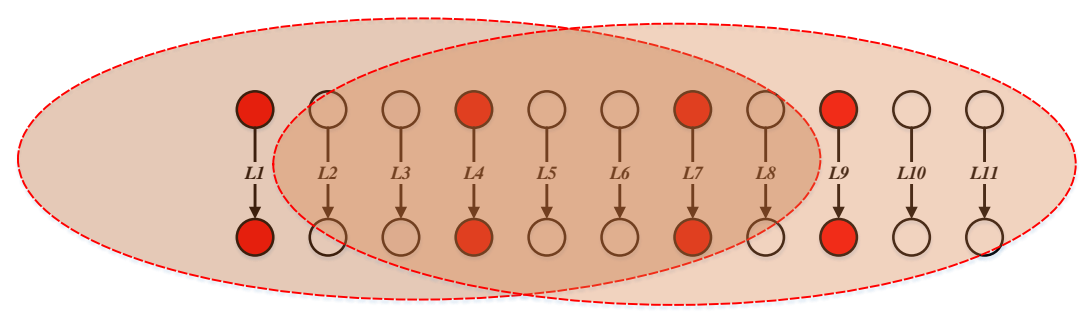

Figure D.9: The starvation example in channel 1

ure D.8. Among all the links using channel 1, links $L 1$ and $L 9$ are the two border links that are out of each other's carrier sensing range. Hence, border effect exists and causes flow starvation on the middle links L4 and L7 between two border links. Once starvation exists, the fairness of this WBN degrades significantly.

In all, we find that: (i) flow starvation leads to severe unfairness that is caused by global interference between border links and middle links, and (ii) CA algorithms cannot prevent flow starvation in ineffective carrier sensing scenarios if the interference model used to select interference sets only consider local interference between a link and its neighbouring links. Hence, CA algorithms need a new interference model reflecting global interference to prevent flow starvation and improve fairness. 
208APPENDIX D. INVESTIGATING FAIRNESS WITH CA ALGORITHM 


\section{Appendix E}

\section{Investigation Goodput and Fairness with Channel}

\section{Assignment Algorithms in IEEE 802.11 WBNs}

This chapter investigates the trade-off between goodput and fairness with limited channel resources in IEEE 802.11 WBNs. By analysing goodput and fairness through simulation with different CA algorithms, we discuss the trade-off between goodput and fairness and identify the strengths of these three CA algorithms in achieving goodput and fairness. The findings in this section inform the design of the proposed multi-objective CA algorithm in Chapter 5 .

\section{E.1 Simulation Environments}

Three classes of CA algorithms are selected to represent different strategies of channel allocation: (i) a standard clique-based CA algorithm INSTC [140] (more details in Section 4.2.2.4 on page 95), (ii) network partitioning CA (NPCA) algorithm [32,130] (more details in Section4.2.1), 
and (iii) the traffic-unaware anti-starvation CA (TUASCA) algorithm [117] (more details in Section 4.2.1).

The inputs of the three $\mathrm{CA}$ algorithms and simulation configurations are the same as those in Section 4.2.3.1. The measurement metrics of network goodput and fairness include normalised average goodput and total goodput, Jain's index [33], and starvation link ratio. Their definitions are listed in Section 4.2.3.1 on page 97

The results shown for the average goodput in this section are calculated from 50 randomly seeded simulation runs. All averages shown are reported with confidence interval of $95 \%$ given by the range of $[5.2,15.66]$ kbps under the assumption that the averages are normally distributed.

The next sections analyse fairness and goodput with the three selected CA algorithms through simulation.

\section{E.2 Analysis of Fairness}

Figure E.1 are the results of Jain's index from three CA algorithms with $95 \%$ confidence interval in grid topologies. When the border distance is less than the carrier sensing range (e.g. $200 \mathrm{~m}$ and $400 \mathrm{~m}$ ), three CA algorithms achieve similar fairness that is between 0.9 and 1.0. It shows that in effective carrier sensing scenarios, fairness remains a high level because the carrier sensing mechanism provides a fair sharing of channel resource among all links.

However, in ineffective carrier sensing scenarios, the NPCA and TUASCA algorithms achieve high Jain's index while INSTC achieves lowest Jain's index.

Figure E.2 is the result of link starvation ratio of three CA algorithms to further study fairness. NPCA and TUASCA do not have any flow starvation and thus explain their high fairness in Figure E.1. However, flow starvation exists in INSTC algorithm in ineffective carrier sensing scenarios that leads to low Jain's index. 


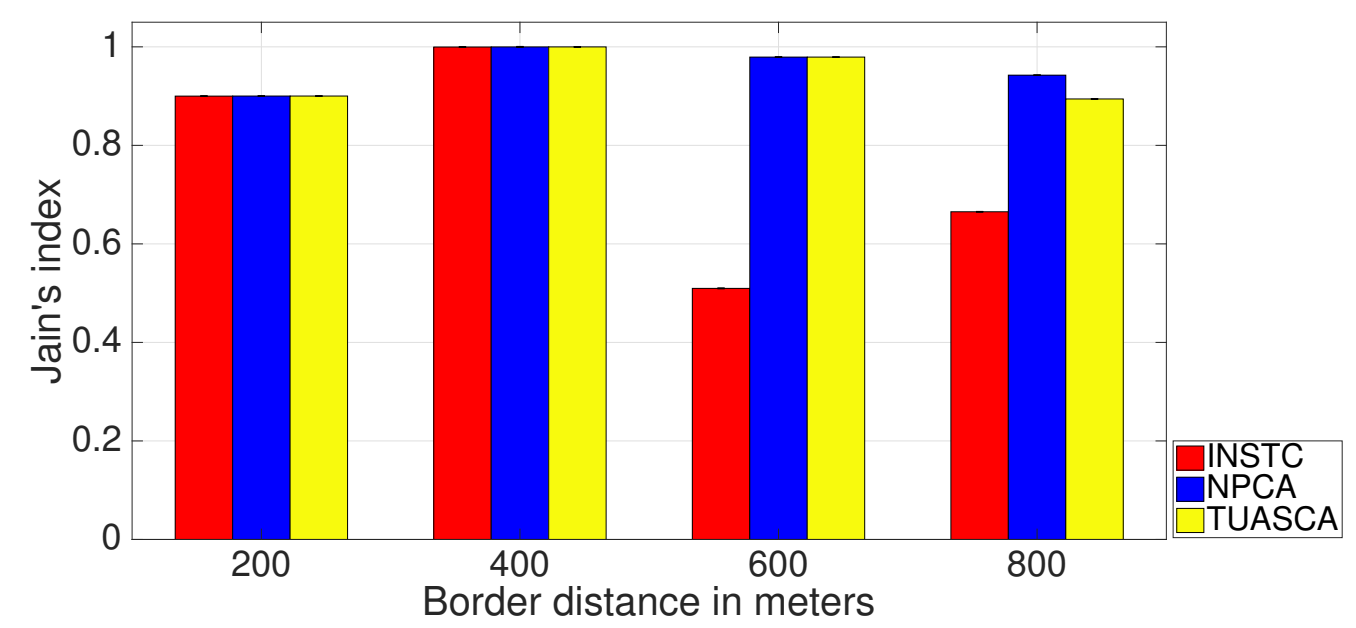

Figure E.1: The comparison of fairness index in grid topologies

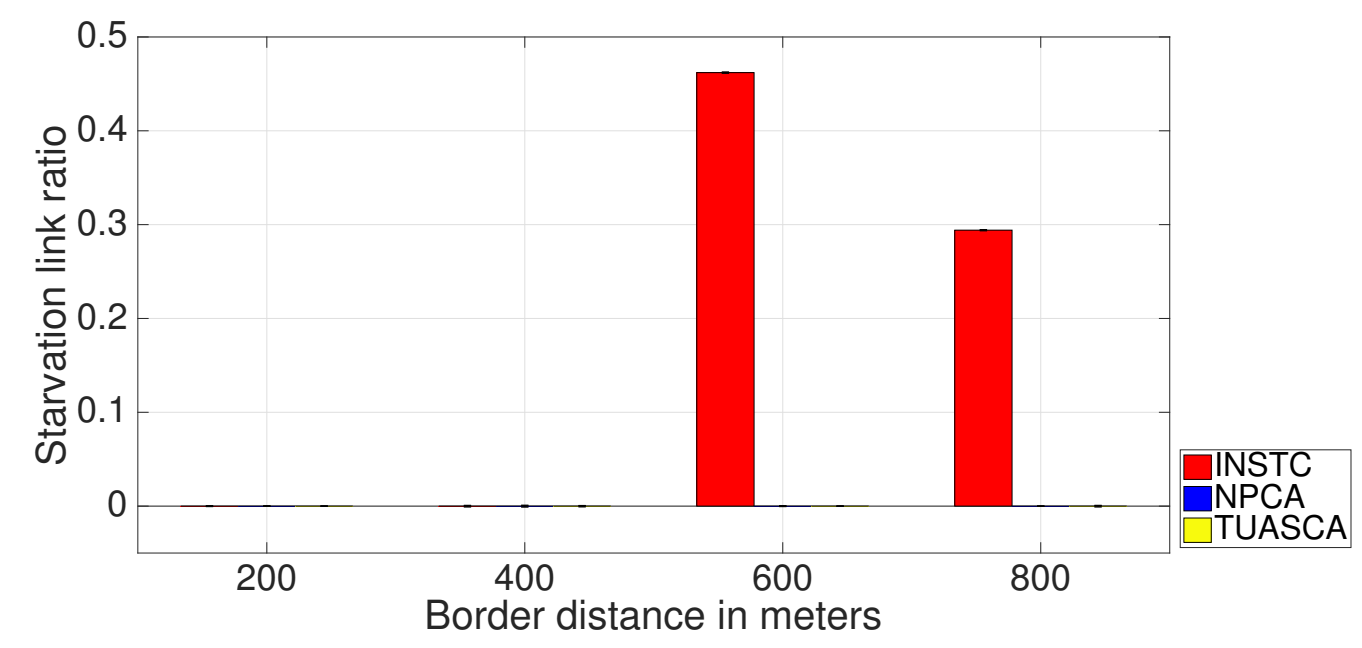

Figure E.2: The comparison of starvation link ratio

\section{E.3 Analysis of Goodput}

In this section, we compare two goodput metrics of three CA algorithms. Figure E.3 is the results of average goodput and total goodput from three CA algorithms with corresponding 95\% confidence interval. In the fig- 


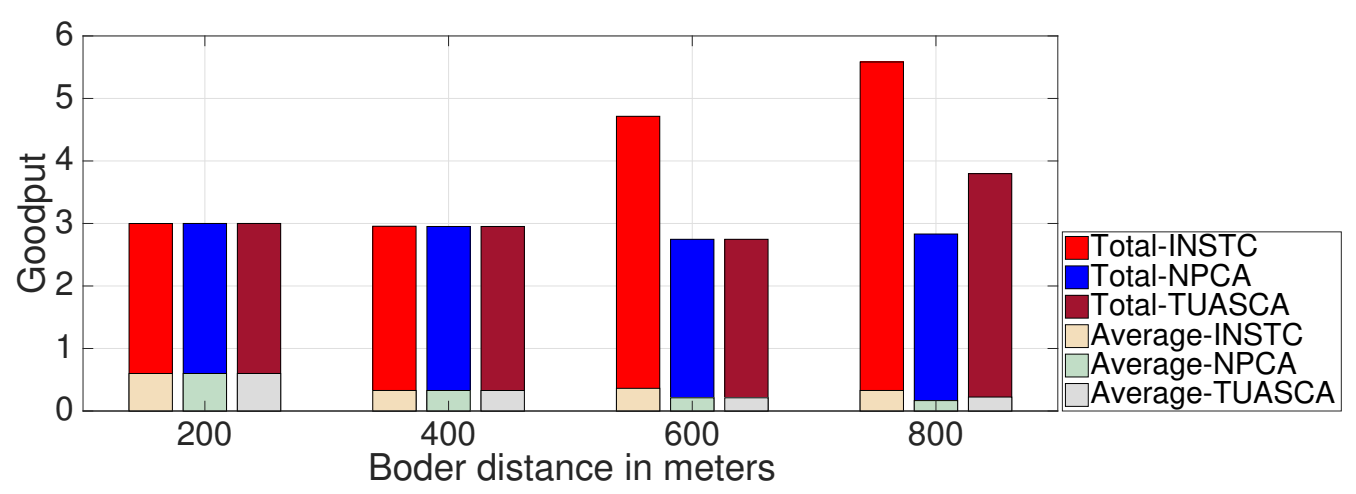

Figure E.3: The comparison of average and total goodput under effective carrier sensing

ure, the $\mathrm{X}$ axis refers to the network size while the $\mathrm{Y}$ axis represents the normalised average goodput and total goodput with respect of the maximum net capacity. In the effective carrier sensing scenarios (e.g. $200 \mathrm{~m}$ and $400 \mathrm{~m}$ ), the total goodput remains constant and three CA algorithms achieve the same average goodput for four network sizes. The results meets the expectation that all nodes share the channel capacity without channel reuse in effective carrier sensing scenarios. Also, the average goodput reduces with the increase of link number.

For ineffective carrier sensing scenarios (e.g. $600 \mathrm{~m}$ and $800 \mathrm{~m}$ ), Figure E.3 shows a different pattern from effective carrier sensing scenarios. The total goodput of INSTC algorithm gradually increases while NPCA algorithm remains a constant total goodput and TUASCA is the middle between INSTC and NPCA in the $800 \mathrm{~m}$ scenario. The corresponding average goodput has the same pattern, INSTC is the highest, and TUASCA is medium and NPCA is the lowest. The INSTC algorithm achieves the highest goodput because it reuses three channels and the total goodput reaches to the two times of three channel capacity. TUASCA partially reuses channel resources and achieves higher goodput than NPCA that does not reuse any channel resource. 


\section{E.4 Discussion}

From the simulation results, we find that the trade-off exists between goodput and fairness. High goodput is a function of channel reuse while fairness is governed by the presence of flow starvation. The analysis herein indicates that high goodput and high fairness are a dichotomy with two seemingly opposite goals under limited channel condition.

Different CA algorithms have different strengths and weakness in achieving goodput and fairness. In effective carrier sensing scenarios, all three CA algorithms achieve identical goodput and fairness. However, in the ineffective carrier sensing scenario $(D=800 \mathrm{~m})$, INSTC achieves the highest goodput but poor fairness, NPCA achieves highest fairness but poor goodput while TUASCA's performance is somewhere in the middle ( $20 \%$ higher goodput compared to NPCA and 32\% higher fairness compared to INSTC). Moreover, these three CA algorithms lack a fitness function to evaluate the potential performance of the output of channel allocation and cannot justify whether the output can meet the desired QoS requirement or not.

Ideally, we can evaluate the performance of the three different CA algorithms and tune them for fairness and goodput that is superior to each CA algorithm individually. 
214 APPENDIX E. INVESTIGATING GOODPUT AND FAIRNESS 


\section{Appendix F}

\section{Additional Validation of Multi-objective Channel Assignment Algorithms in Random Topologies}

This chapter discusses the validation of the traffic-unaware and trafficaware multi-objective channel assignment algorithms using random topologies. Random topologies we use are generated by a R script. With a given area and given link number, the node position and link length are generated randomly according to uniform distribution. The network size of a random topology is set as $D=800 \mathrm{~m}$ with 17 links and the transmitterreceiver separation is less than $50 \mathrm{~m}$.

The inputs of the three CA algorithms and simulation configurations are the same as those in Section 5.2.2 on page 150. Simulation results shown in this section are averages from 100 randomly seeded simulation runs. All averages of simulation results shown are reported with confidence interval of $95 \%$ with the range of $[7.5,10.24] \mathrm{kbps}$ under the assumption that the averages are normally distributed.

The next sections discuss the validation of TUMOCA and TAMOCA in 
random topologies separately.

\section{F.1 Validation of Traffic-unaware Multi-objective Channel Assignment in Random Topologies}

First, the predefined QoS requirement is set as $S R_{\text {Thre }}=0.0, F I_{\text {Thre }}=$ 0.7 , and $A G_{\text {Thre }}=0.2$. The results from the TUMOCA algorithm using three and four channels are listed in Tables F.1 to F.4. These four tables list the solution set from the TUMOCA algorithm with goodput and fairness requirements respectively. The $C h_{\text {need }}$ column represent the channel count needed in the corresponding allocation. The "Validation" column shows whether the solution in the row meets the corresponding requirement.

For this scenario $(D=800 \mathrm{~m})$ with three channels, the TUMOCA yields one solution that the number of used channels is three. When the count of available channels is increased from three to four with the same QoS requirement and this time TUMOCA algorithm yields three channel allocations in Tables F.3 and F.4.

The simulation results in this section show that TUMOCA algorithm performs well in random topologies. First, simulation results show that all the solutions from the TUMOCA algorithm meet the pre-defined QoS requirements. When using four channels, the TUMOCA also selects the solution using three channels into the solution set. The TUMOCA finds a set of feasible solutions instead of one feasible solution. Third, the prediction of goodput and fairness from the fitness function embedded in the TUMOCA algorithm is very close to the simulation results. 
Table F.1: The solution set for a $D=800 \mathrm{~m}$ random topology with three available channels and goodput requirement

\begin{tabular}{c|c|cccc}
\hline Index & $C h_{\text {need }}$ & $A G_{F E A}$ & $A G_{S}$ & $A G_{\text {Thre }}$ & Validation \\
\hline TUMOCA\#1 & 3 & {$[0.226,0.236]$} & 0.246 & 0.2 & $\checkmark$ \\
\hline
\end{tabular}

Table F.2: The solution set for a $D=800 \mathrm{~m}$ random topology with three available channels and fairness requirements

\begin{tabular}{c|c|cccc|cccc}
\hline Index & $C h_{\text {need }}$ & $S R_{F E A}$ & $S R_{S}$ & $S R_{\text {Thre }}$ & Validation & $F I_{\text {FEA }}$ & $F I_{S}$ & $F I_{\text {Thre }}$ & Validation \\
\hline TUMOCA\#1 & 3 & 0.00 & 0.00 & 0.0 & $\checkmark$ & {$[0.826,0.835]$} & 0.824 & 0.7 & $\checkmark$ \\
\hline
\end{tabular}

Table F.3: The solution set for a $D=800 \mathrm{~m}$ random topology with four available channels and goodput requirements

\begin{tabular}{c|c|cccc}
\hline Index & $C h_{\text {need }}$ & $A G_{\text {FEA }}$ & $A G_{s}$ & $A G_{\text {Thre }}$ & Validation \\
\hline TUMOCA\#1 & 3 & {$[0.229,0.235]$} & 0.223 & 0.2 & $\checkmark$ \\
TUMOCA\#2 & 4 & {$[0.224,0.235]$} & 0.218 & 0.2 & $\checkmark$ \\
TUMOCA\#3 & 4 & {$[0.285,0.294]$} & 0.276 & 0.2 & $\checkmark$ \\
\hline
\end{tabular}

Table F.4: The solution set for a $D=800 \mathrm{~m}$ random topology with four available channels and fairness requirements

\begin{tabular}{c|c|cccc|cccc}
\hline Index & $C h_{\text {need }}$ & $S R_{\text {FEA }}$ & $S R_{S}$ & $S R_{\text {Thre }}$ & Validation & $F I_{\text {FEA }}$ & $F I_{S}$ & $F I_{\text {Thre }}$ & Validation \\
\hline TUMOCA\#1 & 3 & 0.000 & 0.000 & 0.0 & $\checkmark$ & {$[0.897,0.911]$} & 0.895 & 0.7 & $\checkmark$ \\
TUMOCA\#2 & 4 & 0.000 & 0.000 & 0.0 & $\checkmark$ & {$[0.991,0.991]$} & 0.983 & 0.7 & $\checkmark$ \\
TUMOCA\#3 & 4 & 0.000 & 0.000 & 0.0 & $\checkmark$ & {$[0.975,0.980]$} & 0.977 & 0.7 & $\checkmark$ \\
\hline
\end{tabular}




\section{F.2 Validation of Traffic-aware Multi-objective Chan- nel Assignment in Random Topologies}

This section shows the validation of the TAMOCA algorithm in random topologies through simulation. Similar to the validation of TUMOCA algorithm, the examples of the TAMOCA algorithm only show the validation in ineffective carrier sensing scenarios $(D=800 \mathrm{~m}$ with 17 links). The simulation runs with Qualnet 5.2 by using the parameters in Table 5.8 .

As TAMOCA algorithm is a traffic-aware $C A$ algorithm, the validation will be conducted in heavy traffic condition $(f(i)=0.8)$ and medium traffic condition $(f(i)=0.4)$ separately.

\section{F.2.1 Validation of Traffic-aware Multi-objective Channel Assignment in random topologies under heavy traffic condition $(f(i)=0.8)$}

Table F.5: The solution set for a $D=800 \mathrm{~m}$ random topology with four available channels and goodput requirements $(f(i)=0.8)$

\begin{tabular}{c|c|cccc}
\hline Index & $C h_{\text {need }}$ & $A G_{F E A}$ & $A G_{s}$ & $A G_{\text {Thre }}$ & Validation \\
\hline TAMOCA\#1 & 3 & {$[0.226,0.235]$} & 0.223 & 0.2 & $\checkmark$ \\
TAMOCA\#2 & 4 & {$[0.224,0.235]$} & 0.218 & 0.2 & $\checkmark$ \\
TAMOCA\#3 & 4 & {$[0.285,0.294]$} & 0.277 & 0.2 & $\checkmark$ \\
\hline
\end{tabular}

This subsection validates the TAMOCA algorithm by selecting the traffic demand as 0.8 that is normalised traffic demand with respect of maximum net bandwidth.

First, the predefined QoS requirements is set as $S R_{\text {Thre }}=0.0, F I_{\text {Thre }}=$ 0.70 , and $A G_{\text {Thre }}=0.2$. Tables F.5 to F.6 provides the list of the solution set from the TAMOCA algorithm with goodput and fairness requirements 
Table F.6: The solution set for a $D=800 \mathrm{~m}$ random topology with four available channels and fairness requirements $(f(i)=0.8)$

\begin{tabular}{c|c|cccc|cccc}
\hline Index & $C h_{\text {need }}$ & $S R_{\text {FEA }}$ & $S R_{S}$ & $S R_{\text {Thre }}$ & Validation & $F I_{\text {FEA }}$ & $F I_{s}$ & $F I_{\text {Thre }}$ & Validation \\
\hline TAMOCA\#1 & 3 & 0.000 & 0.000 & 0.0 & $\checkmark$ & {$[0.826,0.835]$} & 0.894 & 0.7 & $\checkmark$ \\
TAMOCA\#2 & 4 & 0.000 & 0.000 & 0.0 & $\checkmark$ & {$[0.991,0.991]$} & 0.983 & 0.7 & $\checkmark$ \\
TAMOCA\#3 & 4 & 0.000 & 0.000 & 0.0 & $\checkmark$ & {$[0.975,0.980]$} & 0.977 & 0.7 & $\checkmark$ \\
\hline
\end{tabular}

using four channels respectively. The $C h_{\text {need }}$ column represent the channel count needed in the corresponding allocation. The "validation" column shows whether the solution in the row meets the corresponding requirement.

The TAMOCA yields three solutions using four channels. When the traffic demand is set as $f(i)=0.8$, this ineffective carrier sensing scenario $(D=800 \mathrm{~m})$ still has the potential risk of border effect and flow starvation. Simulation results show that all the solutions from the TAMOCA meet the pre-defined QoS requirement with accurate prediction from the fitness function. The same as TUMOCA algorithm, the TAMOCA algorithm also includes all the feasible solution in the solution set. In addition, the TAMOCA algorithm meets the desired outcome of finding multiple feasible solutions.

\section{F.2.2 Validation of Traffic-aware Multi-objective Channel Assignment in random topologies under medium traf- fic condition $(f(i)=0.4)$}

This subsection validates the TAMOCA by selecting the normalised traffic demand with respect to the maximum net bandwidth as 0.4 for all links in a given $W B N$.

First, the predefined QoS requirements as $S R_{\text {Thre }}=0.0, F I_{\text {Thre }}=0.7$, and $A G_{\text {Thre }}=0.2$. Tables F.7 and F.8 provides the list of the solution set from the TAMOCA algorithm with goodput and fairness requirements us- 
ing four channels respectively.

Table F.7: The solution set for a $D=800 \mathrm{~m}$ grid topology with four available channels and goodput requirements $(f(i)=0.4)$

\begin{tabular}{c|c|cccc}
\hline Index & $C h_{\text {need }}$ & $A G_{F E A}$ & $A G_{S}$ & $A G_{\text {Thre }}$ & Validation \\
\hline TAMOCA\#1 & 3 & {$[0.207,0.224]$} & 0.232 & 0.2 & $\checkmark$ \\
TAMOCA\#2 & 4 & {$[0.306,0.365]$} & 0.340 & 0.2 & $\checkmark$ \\
TAMOCA\#3 & 4 & {$[0.224,0.235]$} & 0.218 & 0.2 & $\checkmark$ \\
TAMOCA\#4 & 4 & {$[0.285,0.294]$} & 0.283 & 0.2 & $\checkmark$ \\
\hline
\end{tabular}

Table F.8: The solution set for a $D=800 \mathrm{~m}$ grid topology with four available channels and fairness requirements $(f(i)=0.4)$

\begin{tabular}{c|c|cccc|cccc}
\hline Index & $C h_{\text {need }}$ & $S R_{\text {FEA }}$ & $S R_{S}$ & $S R_{\text {Thre }}$ & Validation & $F I_{\text {FEA }}$ & $F I_{S}$ & $F I_{\text {Thre }}$ & Validation \\
\hline TAMOCA\#1 & 3 & 0.000 & 0.000 & 0.0 & $\checkmark$ & {$[0.900,0.930]$} & 0.895 & 0.7 & $\checkmark$ \\
TAMOCA\#2 & 4 & 0.000 & 0.000 & 0.0 & $\checkmark$ & {$[0.958,0.965]$} & 0.957 & 0.7 & $\checkmark$ \\
TAMOCA\#3 & 4 & 0.000 & 0.000 & 0.0 & $\checkmark$ & {$[0.991,0.991]$} & 0.964 & 0.7 & $\checkmark$ \\
TAMOCA\#4 & 4 & 0.000 & 0.000 & 0.0 & $\checkmark$ & {$[0.907,0.917]$} & 0.896 & 0.7 & $\checkmark$ \\
\hline
\end{tabular}

Using four channels, the TAMOCA algorithm yields four solutions and simulation results show all solutions meet the desired performance for planning IEEE 802.11 WBNs. With the decrease of the traffic demand, the capacity contention reduces so that the TAMOCA algorithm finds more suitable solutions for IEEE 802.11 WBN planning.

The simulation results in this section show that TAMOCA algorithm performs well in random topologies with heavy and medium traffic demands. All the solutions meet the pre-defined requirements. 


\section{Bibliography}

[1] Cisco wireless mesh access points, design and deployment guide, release 7.5. Retrieved from https://www.cisco.com/c/en/ us/td/docs/wireless/technology/mesh/7-5/design/ guide/mesh75/mesh74_chapter_011.html. Accessed: 201708-07.

[2] IEEE standard for Wireless LAN Medium Access Control (MAC) and Physical Layer (PHY) specifications. IEEE Std 802.11-1997 (Nov 1997), 1-445.

[3] IEEE standard for the functional architecture of next generation service overlay networks. IEEE Std 1903-2011 (Oct 2011), 1-147.

[4] Backhaul technologies for small cells user cases, requirements and solutions. Small Cell Forum, UK Report version: 049.01.01.19 (2013).

[5] Akyildiz, I. F., Lee, W.-Y., Vuran, M. C., And Mohanty, S. $\mathrm{NeXt}$ generation/dynamic spectrum access/cognitive radio wireless networks: a survey. Computer Networks, Elsevier 50, 13 (2006), 2127-2159.

[6] AKyILdiZ, I. F., WANG, X., AND WANG, W. Wireless mesh networks: a survey. Computer networks, Elsevier 47, 4 (2005), 445-487.

[7] Al Islam, A. A., Islam, M. J., Nurain, N., and RaghuNATHAN, V. Channel assignment techniques for multi-radio wire- 
less mesh networks: A survey. IEEE Communications Surveys Tutorials 18, 2 (2016), 988-1017.

[8] Alicherry, M., BhatiA, R., AND Li, L. E. Joint channel assignment and routing for throughput optimization in multi-radio wireless mesh networks. In Proceedings of the 11th Annual International Conference on Mobile Computing and Networking (Cologne, Germany, 2005), MobiCom '05, ACM, pp. 58-72.

[9] Amaldi, E., Capone, A., And Malucelli, F. Planning UMTS base station location: Optimization models with power control and algorithms. IEEE Transactions on Wireless Communications 2, 5 (2003), 939-952.

[10] Anderton, W. C., AND YounG, M. Is our model for contention resolution wrong?: Confronting the cost of collisions. In Proceedings of the 29th ACM Symposium on Parallelism in Algorithms and Architectures (Washington, DC, USA, 2017), SPAA '17, ACM, pp. 183-194.

[11] Athota, K., AND NeGI, A. A topology-aware contributory load based channel assignment algorithm for WMN. In Proceedings of the 11th International Conference on Wireless and Optical Communications Networks (Andhra Pradesh,India, 2014), WOCN'14, IEEE, pp. 1-5.

[12] Audhya, G. K., Sinha, K., Ghosh, S. C., And Sinha, B. P. A survey on the channel assignment problem in wireless networks. Wireless Communications and Mobile Computing, Wiley Online Library 11, 5 (2011), 583-609.

[13] Avallone, S., AND Akyildiz, I. F. A channel assignment algorithm for multi-radio wireless mesh networks. Computer Communications, Elsevier 31, 7 (2008), 1343-1353.

[14] Avallone, S., AND Di Stasi, G. Design and implementation of WiMesh: A tool for the performance evaluation of multi-radio wire- 
less mesh networks. Journal of Network and Computer Applications, Elsevier 63 (2016), 98-109.

[15] Avallone, S., Stasi, G., AND Kassler, A. A traffic-aware channel and rate reassignment algorithm for wireless mesh networks. IEEE Transactions on Mobile Computing 12, 7 (2013), 1335-1348.

[16] Avonts, J., AND Blondia, C. A framework to compare topology algorithms in multi-channel multi-radio wireless mesh networks. Computer Networks, Elsevier 98 (2016), 89-108.

[17] B̈̈CK, T., Fogel, D. B., AND MiChalewiCZ, Z. Handbook of evolutionary computation. New York: Oxford (1997).

[18] BAILEY, D. Practical radio engineering and telemetry for industry. Elsevier, 2003.

[19] BAKHSHI, B., AND KHORSANDI, S. A maximum fair bandwidth approach for channel assignment in wireless mesh networks. In Proceedings of the Wireless Communications and Networking Conference (Los Angeles, CA, USA, 2008), WCNC'08, IEEE, pp. 2176-2181.

[20] BAKHSHI, B., AND KHORSANDI, S. A maximum fair bandwidth approach for channel assignment in wireless mesh networks. In proceedings of Wireless Communications and Networking Conference (Las Vegas, NV, USA, March 2008), WCNC'08, IEEE, pp. 2176-2181.

[21] BAKHSHI, B., AND KHORSANDI, S. On the performance and fairness of dynamic channel allocation in wireless mesh networks. International Journal of Communication Systems, Wiley Online Library 26, 3 (2013), 293-314.

[22] BaKhshi, B., Khorsandi, S., And CAPOnE, A. On-line joint QoS routing and channel assignment in multi-channel multi-radio wireless mesh networks. Computer Communications, Elsevier 34, 11 (2011), 1342-1360. 
[23] Barolli, A., XhafA, F., And TAKizawa, M. Optimization problems and resolution methods for node placement in wireless mesh networks. In Proceedings of the 14th International Conference onNetwork-Based Information Systems (Tirana, Albania, 2011), NBiS'11, IEEE, pp. 126-134.

[24] Bellalta, B., Bononi, L., Bruno, R., And Kassler, A. Next generation IEEE 802.11 wireless local area networks: Current status, future directions and open challenges. Computer Communications, Elsevier 75 (2016), 1 - 25.

[25] Benyamina, D., Hafid, A., And Gendreau, M. Wireless mesh networks design- a survey. IEEE Communications Surveys Tutorials 14, 2 (Second 2012), 299-310.

[26] Benyamina, D., Hafid, A., Gendreau, M., Maureira, J., ET AL. On the design of reliable wireless mesh network infrastructure with QOS constraints. Computer Networks, Elsevier 55, 8 (2011), 1631-1647.

[27] BIANCHI, G. Performance analysis of the IEEE 802.11 distributed coordination function. IEEE Journal on Selected Areas in Communications 18, 3 (2000), 535-547.

[28] Bianchi, G., And TinniRello, I. Remarks on IEEE 802.11 DCF performance analysis. IEEE Communications Letters 9, 8 (2005), 765767.

[29] Bicket, J., Aguayo, D., Biswas, S., And Morris, R. Architecture and evaluation of an unplanned $802.11 \mathrm{~b}$ mesh network. In Proceedings of the 11th Annual International Conference on Mobile Computing and Networking (Cologne, Germany, 2005), MobiCom '05, ACM, pp. 31-42. 
[30] Boorstyn, R. R., Kershenbaum, A., Maglaris, B., AND SAhin, $\mathrm{V}$. Throughput analysis in multihop CSMA packet radio networks. IEEE Transactions on Communications 35, 3 (1987), 267-274.

[31] Bosio, S., CAPONE, A., AND CeSANA, M. Radio planning of wireless local area networks. IEEE/ACM Transactions on Networking 15, 6 (2007), 1414-1427.

[32] Brzezinski, A., Zussman, G., AND Modiano, E. Enabling distributed throughput maximization in wireless mesh networks: A partitioning approach. In Proceedings of the 12th Annual International Conference on Mobile Computing and Networking (Los Angeles, CA, USA, 2006), MobiCom '06, ACM, pp. 26-37.

[33] BUKH, P. N. D., AND JAIN, R. The art of computer systems performance analysis, techniques for experimental design, measurement, simulation and modeling. JSTOR, 1992.

[34] Calì, F., Conti, M., And Gregori, E. Dynamic tuning of the IEEE 802.11 protocol to achieve a theoretical throughput limit. IEEE/ACM Transactions on Networking (ToN) 8, 6 (2000), 785-799.

[35] Chandrasekhar, V., Andrews, J. G., And Gatherer, A. Femtocell networks: a survey. IEEE Communications Magazine 46, 9 (September 2008), 59-67.

[36] Chaudhry, A. U., Ahmad, N., And Hafez, R. H. M. Improving throughput and fairness by improved channel assignment using topology control based on power control for multi-radio multichannel wireless mesh networks. EURASIP Journal on Wireless Communications and Networking, Springer 2012, 1 (Apr 2012), 155.

[37] Chaudhry, A. U., Hafez, R. H., and Chinneck, J. W. On the impact of interference models on channel assignment in multi-radio 
multi-channel wireless mesh networks. Ad Hoc Networks, Elsevier 27 (2015), 68-80.

[38] Chavez, K., Goratti, L., Rasheed, T., Oljira, D. B., Fedrizzi, R., AND Riggio, R. The Evolutionary Role of Communication Technologies in Public Safety Networks 1: Overview and Challenges. Elsevier, 2015.

[39] Chen, X., XU, J., YuAN, W., LiU, W., And ChenG, W. Channel assignment in heterogeneous multi-radio multi-channel wireless networks: A game theoretic approach . Computer Networks, Elsevier 57, 17 (2013), 3291-3299.

[40] Cheng, H., XiOng, N., YAnG, L. T., Chen, G., Zhuang, X., AND LEE, C. Links organization for channel assignment in multi-radio wireless mesh networks. Multimedia tools and applications, Springer 65, 2 (2013), 239-258.

[41] Chlebus, B. S., Kowalski, D. R., And RadzIK, T. Many-toMany Communication in Radio Networks. Algorithmica, Springer 54, 1 (2009), 118-139.

[42] Clark, B. N., Colbourn, C. J., And Johnson, D. S. Unit disk graphs. Annals of Discrete Mathematics, Elsevier 48 (1991), 165-177.

[43] Coldrey, M., Engström, U., Helmersson, K. W., Hashemi, M., Manholm, L., And Wallentin, P. Wireless backhaul in future heterogeneous networks. Ericsson Review, Ericsson 91 (2014).

[44] Damnjanovic, A., Montojo, J., Wei, Y., Ji, T., Luo, T., VAJAPEYAM, M., YoO, T., SONG, O., AND MALladi, D. A survey on 3GPP heterogeneous networks. IEEE Wireless Communications 18, 3 (June 2011), 10-21. 
[45] Daneshgaran, F., Laddomada, M., Mesiti, F., And Mondin, M. Unsaturated throughput analysis of IEEE 802.11 in presence of non ideal transmission channel and capture effects. IEEE Transactions on Wireless Communications 7, 4 (2008), 1276-1286.

[46] DAS, B., AND RoY, S. Load Balancing Techniques for Wireless Mesh Networks: A Survey. In Proceedings of the International Symposium on Computational and Business Intelligence (New Delhi, India, 2013), ISCBI'13, IEEE, pp. 247-253.

[47] Das, S. M., Pucha, H., Koutsonikolas, D., Hu, Y. C., AND PEROULIS, D. DMesh: incorporating practical directional antennas in multichannel wireless mesh networks. IEEE Journal on Selected Areas in Communications 24, 11 (2006), 2028-2039.

[48] DenG, J., Liang, B., And VARShney, P. K. Tuning the carrier sensing range of IEEE 802.11 MAC. In Proceedings of the Global Telecommunications Conference (Dallas, Texas, USA, June 2004), GLOBECOM '04, IEEE, pp. 1-8.

[49] Dhillon, H. S., AND CAIRE, G. Wireless backhaul networks: Capacity bound, scalability analysis and design guidelines. IEEE Transactions on Wireless Communications 14, 11 (2015), 6043-6056.

[50] Dhillon, H. S., AND CAIRE, G. Wireless backhaul networks: Capacity bound, scalability analysis and design guidelines. IEEE Transactions on Wireless Communications 14, 11 (Nov 2015), 6043-6056.

[51] Ding, R., XUE, K., HONG, P., AND Du, Z. A novel cluster-based channel assignment scheme for wireless mesh networks. In Proceedings of the Consumer Communications and Networking Conference (Las Vegas, NV, USA, 2012), CCNC'12, IEEE, pp. 921-925.

[52] Doraghinejad, M., Nezamabadi-Pour, H., and Mahani, A. Channel assignment in multi-radio wireless mesh networks using 
an improved gravitational search algorithm. Journal of Network and Computer Applications, Elsevier 38 (2014), 163-171.

[53] Draves, R., PADhye, J., AND Zill, B. Routing in multi-radio, multi-hop wireless mesh networks. In Proceedings of the 10th Annual International Conference on Mobile Computing and Networking (Philadelphia, PA, USA, 2004), MobiCom '04, ACM, pp. 114-128.

[54] Durvy, M., Dousse, O., AND Thiran, P. Border effects, fairness, and phase transition in large wireless networks. In Proceedings of the 27th Conference on Computer Communications (Phoenix, AZ, USA, 2008), INFOCOM'08, IEEE, pp. 1274-1282.

[55] ERgen, M., AND VARAIYA, P. Throughput analysis and admission control for IEEE 802.11a. Mobile Networks and Applications, Springer 10,5 (2005), 705-716.

[56] FAnG, X., MisRa, S., Xue, G., AND YAnG, D. The new and improved power grid: A survey. IEEE Communications Surveys Tutorials 14, 4 (Fourth 2012), 944-980.

[57] FELEMBAN, E., AND EKICI, E. Single hop IEEE 802.11 DCF analysis revisited: Accurate modeling of channel access delay and throughput for saturated and unsaturated traffic cases. IEEE Transactions on Wireless Communications 10, 10 (2011), 3256-3266.

[58] Franklin, A. A., Bukkapatanam, V., And Murthy, C. On the end-to-end flow allocation and channel assignment in multi-channel multi-radio wireless mesh networks with partially overlapped channels. Computer Communications, Elsevier 34, 15 (2011), 1858-1869.

[59] FU, L., LIEW, S. C., AND HUANG, J. Effective carrier sensing in CSMA networks under cumulative interference. IEEE Transactions on Mobile Computing 12, 4 (2013), 748-760. 
[60] Gabale, V., Raman, B., Dutta, P., And Kalyanraman, S. A classification framework for scheduling algorithms in wireless mesh networks. IEEE Communications Surveys Tutorials 15, 1 (2013), 199222.

[61] Gambiroza, V., Sadeghi, B., AND Knightly, E. W. End-to-end performance and fairness in multihop wireless backhaul networks. In Proceedings of the 10th Annual International Conference on Mobile Computing and Networking (Philadelphia, PA, USA, 2004), MobiCom '04, ACM, pp. 287-301.

[62] GaO, Y., ChIU, D.-M., AND LuI, J. C. Determining the end-toend throughput capacity in multi-hop networks: Methodology and applications. In Proceedings of the Joint International Conference on Measurement and Modeling of Computer Systems (New York, NY, USA, 2006), SIGMETRICS '06/Performance '06, ACM, pp. 39-50.

[63] Gardellin, V., Das, S. K., Lenzini, L., Cicconetti, C., AND MingOzZI, E. G-PAMELA: A divide-and-conquer approach for joint channel assignment and routing in multi-radio multi-channel wireless mesh networks. Journal of parallel and distributed computing, Elsevier 71, 3 (2011), 381-396.

[64] Garetto, M., Salonidis, T., And Knightly, E. W. Modeling per-flow throughput and capturing starvation in CSMA multi-hop wireless networks. IEEE/ACM Transactions on Networking (ToN) 16, 4 (2008), 864-877.

[65] Ghosh, A., Mangalvedhe, N., Ratasuk, R., Mondal, B., CUDAK, M., Visotsky, E., ThOMAs, T. A., ANDreWs, J. G., XIA, P., Jo, H. S., Dhillon, H. S., AND Novlan, T. D. Heterogeneous cellular networks: From theory to practice. IEEE Communications Magazine 50, 6 (June 2012), 54-64. 
[66] Goldsmith, A. Wireless communications. Cambridge university press, 2005.

[67] GONG, D., ZHAO, M., AND YANG, Y. Channel assignment in multirate 802.11n WLANs. In Proceedings of the Wireless Communications and Networking Conference (Shanghai, China, 2013), WCNC'13, IEEE, pp. 392-397.

[68] Goussevskaia, O., Oswald, Y. A., And Wattenhofer, R. Complexity in geometric SINR. In Proceedings of the 8th ACM International Symposium on Mobile Ad Hoc Networking and Computing (New York, NY, USA, 2007), MobiHoc '07, ACM, pp. 100-109.

[69] Hei, X., Liang, C., Liang, J., LiU, Y., And Ross, K. W. A measurement study of a Large-Scale P2P IPTV system. IEEE Transactions on Multimedia 9, 8 (Dec 2007), 1672-1687.

[70] Ho, I. W.-H., LAM, P. P., CHONG, P. H. J., AND LiEW, S. C. Harnessing the high bandwidth of multiradio multichannel $802.11 \mathrm{n}$ mesh networks. IEEE Transactions on Mobile Computing 13, 2 (2014), 448-456.

[71] HossAIn, E., AND LEUNG, K. K. Wireless mesh networks: architectures and protocols. Springer, 2007.

[72] HuA, C., AND ZHENG, R. Starvation modeling and identification in dense 802.11 wireless community networks. In Proceedings of the 27 th Conference on Computer Communications (Phoenix, AZ, USA, 2008), INFOCOM'08, IEEE, pp. 1696-1704.

[73] HuA, C., AND ZHENG, R. On link-level starvation in dense 802.11 wireless community networks. Computer Networks, Elsevier 54, 17 (2010), 3159-3172. 
[74] HuANG, R., Kim, S., ZHANG, C., AND FANG, Y. Exploiting the capacity of multichannel multiradio wireless mesh networks. IEEE Transactions on Vehicular Technology 58, 9 (2009), 5037-5047.

[75] IYer, A., RosenberG, C., AND KARNIK, A. What is the right model for wireless channel interference? IEEE Transactions on Wireless Communications 8, 5 (2009), 2662-2671.

[76] Jahanshahi, M., Dehghan, M., And Meybodi, M. R. A mathematical formulation for joint channel assignment and multicast routing in multi-channel multi-radio wireless mesh networks. Journal of Network and Computer Applications, Elsevier 34, 6 (2011), 1869-1882.

[77] Jain, K., Padhye, J., Padmanabhan, V. N., AND QiU, L. Impact of interference on multi-hop wireless network performance. Wireless networks, Springer 11, 4 (2005), 471-487.

[78] JAMIL, I., CARIOU, L., AND HElaRD, J. F. Improving the capacity of future IEEE 802.11 high efficiency WLANs. In Proceedings of the 21st International Conference on Telecommunications (Lisbon, Portugal, May 2014), ICT'14, IEEE, pp. 303-307.

[79] Jamshaid, K., Ward, P., Karsten, M., AND Shihada, B. The efficacy of centralized flow rate control in 802.11-based wireless mesh networks. EURASIP Journal on Wireless Communications and Networking, Springer 2013, 1 (Jun 2013), 163.

[80] Jamshaid, K., Ward, P. A., AND Karsten, M. Mechanisms for centralized flow rate control in 802.11-based wireless mesh networks. Computer Networks, Elsevier 56, 2 (2012), 884-901.

[81] Kala, S. M., Musham, R., Reddy, M. P. K., and Tamma, B. R. Reliable prediction of channel assignment performance in wireless mesh networks. In Proceedings of International Conference on Advances 
in Computing, Communications and Informatics (Kerala,India, 2015), ICACCI'15, IEEE, pp. 13-19.

[82] KAlA, S. M., RedDy, M. P. K., AND TAMmA, B. R. Predicting performance of channel assignments in wireless mesh networks through statistical interference estimation. In Proceedings of IEEE International Conference on Electronics, Computing and Communication Technologies (Bangalore, India, 2015), CONECCT'15, IEEE, pp. 1-6.

[83] KHAN, R. H., AND KHAN, J. Y. A comprehensive review of the application characteristics and traffic requirements of a smart grid communications network. Computer Networks, Elsevier 57, 3 (2013), 825-845.

[84] KIM, J., KIM, D., LIM, K.-W., KO, Y.-B., AND LEE, S.-Y. Improving the reliability of IEEE $802.11 \mathrm{~s}$ based wireless mesh networks for smart grid systems. Journal of Communications and Networks, IEEE 14, 6 (2012), 629-639.

[85] KIM, S., CHA, J., AND MA, J. Interference-aware channel assignment with seamless multi-channel monitoring on wireless mesh network. In Proceedings of the 6th Consumer Communications and Networking Conference (Las Vegas, NV, USA, 2009), CCNC'09, IEEE, pp. 1-6.

[86] Ko, B. J., Misra, V., Padhye, J., And Rubenstein, D. Distributed channel assignment in multi-radio 802.11 mesh networks. In Proceedings of the Wireless Communications and Networking Conference (Kowloon, China, 2007), WCNC'07, IEEE, pp. 3978-3983.

[87] Kodialam, M., AND NANDAgOpal, T. Characterizing the capacity region in multi-radio multi-channel wireless mesh networks. In Proceedings of the 11th Annual International Conference on Mobile Computing and Networking (New York, NY, USA, 2005), MobiCom '05, ACM, pp. 73-87. 
[88] Kyasanur, P., And VAidya, N. H. Routing and link-layer protocols for multi-channel multi-interface ad hoc wireless networks. ACM SIGMOBILE Mobile Computing and Communications Review 10, 1 (2006), 31-43.

[89] LEE, J.-F., AND KUO, W.-H. Fairness provisioning in multi-hop wireless backhaul networks: Challenges and solutions. Computer Communications, Elsevier 33, 15 (2010), 1767 - 1772.

[90] Lee, M. W., HWang, G., And Roy, S. Performance modeling and analysis of IEEE 802.11 wireless networks with hidden nodes. In Proceedings of the 16th International Conference on Modeling, Analysis, Simulation of Wireless and Mobile Systems (Barcelona, Spain, 2013), MSWiM '13, ACM, pp. 135-142.

[91] LeNSTRA JR, H. W. Integer programming with a fixed number of variables. Mathematics of operations research, INFORMS 8, 4 (1983), 538-548.

[92] LI, M., AND FENG, Y. Design and implementation of a hybrid channel-assignment protocol for a multi-interface wireless mesh network. IEEE Transactions on Vehicular Technology 59, 6 (July 2010), 2986-2997.

[93] LI, X., WU, J., LIN, S., AND DU, X. Channel switching control policy for wireless mesh networks. Journal of Parallel and Distributed Computing, Elsevier 72, 10 (2012), 1295-1305.

[94] Liaw, Y. S., Dadej, A., And Jayasuriya, A. Performance analysis of IEEE 802.11 DCF under limited load. In Proceedings of the AsiaPacific Conference on Communications (Perth, WA, Australia, 2005), APCC'05, IEEE, pp. 759-763.

[95] LiN, X., AND RASOOL, S. B. Distributed and provably efficient algorithms for joint channel-assignment, scheduling, and routing in 
multichannel ad hoc wireless networks. IEEE/ACM Transactions on Networking (TON) 17, 6 (2009), 1874-1887.

[96] Liu, W., Sirisena, H., PaWlikowski, K., And Willig, A. A new topological index for capacity allocation problem in survivable networks. In Proceedings of the Australasian Telecommunication Networks and Applications Conference (Auckland, New Zealand, 2010), ATNAC'10, IEEE, pp. 25-30.

[97] MA, R., CHEN, H. H., HuAnG, Y. R., AND MENG, W. Smart grid communication: Its challenges and opportunities. IEEE Transactions on Smart Grid 4, 1 (March 2013), 36-46.

[98] Malanchini, I., VAlentin, S., And Aydin, O. Wireless resource sharing for multiple operators: Generalization, fairness, and the value of prediction. Computer Networks, Elsevier 100 (2016), 110-123.

[99] MaliK, A., QAdir, J., AhMad, B., YAU, K.-L. A., And Ullah, U. QoS in IEEE 802.11-based wireless networks: A contemporary review. Journal of Network and Computer Applications, Elsevier 55 (2015), 24-46.

[100] Malnar, M., Neskovic, N., And Neskovic, A. A new quality of service aware multi-channel multi-interface link layer protocol for wireless mesh networks. Wireless Networks, Springer 21, 3 (2015), 727-738.

[101] Marchese, M. QoS Over Heterogeneous Networks. Wiley Publishing, 2007.

[102] Marina, M. K., DAS, S. R., And Subramanian, A. P. A topology control approach for utilizing multiple channels in multi-radio wireless mesh networks. Computer networks, Elsevier 54, 2 (2010), 241-256. 
[103] Martignon, F., Paris, S., Filippini, I., Chen, L., And Capone, A. Efficient and truthful bandwidth allocation in wireless mesh community networks. IEEE/ACM Transactions on Networking, 23, 1 (2015), 161-174.

[104] Medepalli, K., And Tobagi, F. A. Towards performance modeling of IEEE 802.11 based wireless networks: A unified framework and its applications. In Proceedings of the 25th International Conference on Computer Communications (Barcelona, Catalunya, Spain, 2006), INFOCOM'06, IEEE, pp. 1-12.

[105] Methley, S. Essentials of wireless mesh networks. Cambridge University Press, 2009.

[106] Mishra, A. R. Fundamentals of cellular network planning and optimisation: $2 G / 2.5 G / 3 G \ldots$ evolution to $4 G$. Wiley, 2004.

[107] NAVeED, A., KANHERE, S. S., AND JHA, S. K. Topology control and channel assignment in multi-radio multi-channel wireless mesh networks. In Proceedings of the International Conference on Mobile Adhoc and Sensor Systems (Pisa, Italy, 2007), MASS'07, IEEE, pp. 1-9.

[108] NaWAB, F., Jamshaid, K., Shihada, B., AND Ho, P.-H. MAClayer protocol for TCP fairness in Wireless Mesh Networks. In proceedings of the 1st International Conference on Communications in China (Beijing, China, 2012), ICCC'12, IEEE, pp. 448-453.

[109] NG, P. C., AND LIEW, S. C. Throughput analysis of IEEE 802. 11 multi-hop ad hoc networks. IEEE/ACM Transactions on Networking (TON) 15, 2 (2007), 309-322.

[110] Nguyen, S. H., Vu, H. L., AND Andrew, L. L. Performance analysis of IEEE 802.11 WLANs with saturated and unsaturated sources. IEEE Transactions on Vehicular Technology 61, 1 (2012), 333-345. 
[111] OleXA, R. Implementing 802.11, 802.16, and 802.20 Wireless Networks: Planning, Troubleshooting, and Operations. Elsevier, 2004.

[112] PathaK, P. H., AND DutTA, R. A survey of network design problems and joint design approaches in wireless mesh networks. IEEE Communications Surveys Tutorials 13, 3 (Third 2011), 396-428.

[113] Peng, Y., Yu, Y., Guo, L., Jiang, D., And Gai, Q. An efficient joint channel assignment and QOS routing protocol for IEEE 802.11 multi-radio multi-channel wireless mesh networks. Journal of Network and Computer Applications, Elsevier 36, 2 (2013), 843-857.

[114] QU, Y., AND NG, B. The effect of carrier sensing mechanisms on wireless mesh network goodput. In Proceedings of the International Telecommunication Networks and Applications Conference (Sydney, Australia, 2015), ITNAC'15, IEEE, pp. 106-113.

[115] QU, Y., NG, B., AND SEAH, W. A survey of routing and channel assignment in multi-channel multi-radio WMNs. Journal of Network and Computer Applications, Elsevier 65, C (Apr. 2016), 120-130.

[116] QU, Y., NG, B., AND SEAH, W. K. G. A goodput distribution model for IEEE 802.11 wireless mesh networks. In Proceedings of the 34th International Performance Computing and Communications Conference (Nanjing, China, Dec 2015), IPCCC'15, IEEE, pp. 1-8.

[117] QU, Y., NG, B., AND YU, H. Anti-starvation channel assignment with global conflict set selection in IEEE 802.11 wmns. In Proceedings of the 12th Symposium on QoS and Security for Wireless and Mobile Networks (Malta, Malta, 2016), Q2SWinet'16, ACM, pp. 103-110.

[118] Qu, Y., NG, B., YU, H., Andreae, P., AND SeaH, W. K. G. Fitness evaluation for channel assignment algorithms in ieee 802.11 WMNs. 
In Proceedings of the 14th Annual Consumer Communications Networking Conference (Las Vegas, NV, USA, 2017), CCNC'17, IEEE, pp. 361364 .

[119] QueK, T. Q., DE LA Roche, G., GÜVEnç, İ., AND Kountouris, M. Small cell networks: Deployment, $P H Y$ techniques, and resource management. Cambridge University Press, 2013.

[120] RACKLEY, S. A. Wireless networking technology: From principles to successful implementation. Elsevier, 2011.

[121] RAD, A. H. M., AND S. WONG, V. W. Joint optimal channel assignment and congestion control for multi-channel wireless mesh networks. In Proceedings of the International Conference on Communications (Istanbul, Turkey, 2006), ICC'06, IEEE, pp. 1984-1989.

[122] Ramachandran, K. N., Belding, E. M., Almeroth, K. C., AND BUDDHIKOT, M. M. Interference-aware channel assignment in multi-radio wireless mesh networks. In Proceedings of the 25th International Conference on Computer Communications (Barcelona, Spain, 2006), INFOCOM'06, IEEE, pp. 1-12.

[123] Ramamurthi, V., Reaz, A. S., Ghosal, D., Dixit, S., And MUKHERJEE, B. Channel, capacity, and flow assignment in wireless mesh networks . Computer Networks, Elsevier 55, 9 (2011), 2241-2258.

[124] Rangwala, S., Gummadi, R., Govindan, R., and Psounis, $\mathrm{K}$. Interference-aware fair rate control in wireless sensor networks. In ACM SIGCOMM Computer Communication Review (2006), vol. 36, pp. 63-74.

[125] Raniwala, A., AND CKer Chiueh, T. Architecture and algorithms for an IEEE 802.11-based multi-channel wireless mesh network. In Proceedings of the 24th Annual Joint Conference of the Computer 
and Communications Societies (Miami, FL, USA, 2005), INFOCOM'05, IEEE, pp. 2223-2234 vol. 3.

[126] Raniwala, A., Gopalan, K., And Chiueh, T.-C. Centralized channel assignment and routing algorithms for multi-channel wireless mesh networks. ACM SIGMOBILE Mobile Computing and Communications Review 8, 2 (2004), 50-65.

[127] Ray, S., Starobinski, D., And Carruthers, J. B. Performance of wireless networks with hidden nodes: a queuing-theoretic analysis. Computer Communications, Elsevier 28, 10 (2005), 1179-1192.

[128] Riggio, R., Rasheed, T., Testi, S., Granelli, F., And Chlam$\mathrm{TAC}, \mathrm{I}$. Interference and traffic aware channel assignment in WiFibased wireless mesh networks. Ad Hoc Networks, Elsevier 9, 5 (2011), 864-875.

[129] Robinson, J., AND KNightly, E. W. A performance study of deployment factors in wireless mesh networks. In proceedings of the 26th International Conference on Computer Communications (Barcelona, Spain, 2007), INFOCOM'07, IEEE, pp. 2054-2062.

[130] SANCHIS, L. A. Multiple-way network partitioning. IEEE Transactions on Computers 38, 1 (Jan 1989), 62-81.

[131] SHI, H., Prasad, R. V., ONUR, E., AND Niemegeers, I. G. M. M. Fairness in wireless networks:issues, measures and challenges. IEEE Communications Surveys Tutorials 16, 1 (First 2014), 5-24.

[132] SHI, J., SAlONIDIS, T., AND KNIGHTLY, E. W. Starvation mitigation through multi-channel coordination in CSMA multi-hop wireless networks. In Proceedings of the 7th International Symposium on Mobile Ad Hoc Networking and Computing (New York, NY, USA, 2006), MobiHoc '06, ACM, pp. 214-225. 
[133] SI, W., Selvakennedy, S., AND Zomaya, A. Y. An overview of channel assignment methods for multi-radio multi-channel wireless mesh networks. Journal of Parallel and Distributed Computing, Elsevier 70, 5 (2010), 505-524.

[134] Simo Reigadas, J., Martinez-Fernandez, A., Ramos-LopeZ, J., AND SeOAne-Pascual, J. Modeling and optimizing IEEE 802.11 DCF for long-distance links. IEEE Transactions on Mobile Computing 9, 6 (2010), 881-896.

[135] SRIDHAR, S., GuO, J., AND JHA, S. Channel assignment in multiradio wireless mesh networks : A graph-theoretic approach. In Proceedings of the 1st International Communication Systems and Networks and Workshops (Bangalore, India, 2009), COMSNETS'09, IEEE, pp. 110.

[136] SRInivas, M., AND PATNAiK, L. M. Genetic algorithms: a survey. Computer, IEEE 27, 6 (June 1994), 17-26.

[137] Stuedi, P., AND AlONSO, G. Modeling and computing throughput capacity of wireless multihop networks. Computer Networks, Elsevier 52, 1 (2008), 116-129.

[138] Subbaiah, K. V., And Naidu, M. M. An efficient interference aware channel allocation algorithm for wireless mesh networks. In proceedings of the International Conference on Signal Processing and Communication Engineering Systems (Guntur, India, 2015), SPACES'15, IEEE, pp. 416-420.

[139] Subramanian, A. P., Gupta, H., Das, S. R., and CaO, J. Minimum interference channel assignment in multiradio wireless mesh networks. IEEE Transactions on Mobile Computing, 7, 12 (2008), 14591473. 
[140] TANG, J., XUE, G., AND ZHANG, W. Interference-aware topology control and QoS routing in multi-channel wireless mesh networks. In Proceedings of the 6th International Symposium on Mobile Ad Hoc Networking and Computing (Urbana-Champaign, IL, USA, 2005), MobiHoc '05, ACM, pp. 68-77.

[141] TinniRello, I., BIANCHI, G., AND XiaO, Y. Refinements on IEEE 802.11 distributed coordination function modeling approaches. IEEE Transactions on Vehicular Technology 59, 3 (2010), 1055-1067.

[142] Tipmongkolsilp, O., Zaghloul, S., And Jukan, A. The evolution of cellular backhaul technologies: Current issues and future trends. IEEE Communications Surveys Tutorials 13, 1 (2011), 97-113.

[143] Tsertou, A., And Laurenson, D. I. Revisiting the hidden terminal problem in a CSMA/CA wireless network. IEEE Transactions on Mobile Computing 7, 7 (July 2008), 817-831.

[144] Van de Ven, P., Janssen, A. J., And van LeeuwaArden, J. Balancing exposed and hidden nodes in linear wireless networks. IEEE/ACM Transactions on Networking (TON) 22, 5 (2014), 1429-1443.

[145] VAnhatupa, T., HÄNNIKÄINEN, M., AND HÄMÄLÄINEN, T. D. Performance model for ieee $802.11 \mathrm{~s}$ wireless mesh network deployment design. Journal of Parallel and Distributed Computing, Elsevier 68, 3 (2008), 291-305.

[146] Vo, N. S., NGuyen, M. H., HA, D. B., AND Huynh, D. T. Joint distortion aware opportunistic routing and transmission rate assignment for video streaming over wireless mesh networks. In Proceedings of the International Conference on Computing, Management and Telecommunications (Ho Chi Minh City, Vietnam, 2013), ComManTel'13, IEEE, pp. 230-234. 
[147] Vural, S., WeI, D., AND MoEssner, K. Survey of experimental evaluation studies for wireless mesh network deployments in urban areas towards ubiquitous Internet. IEEE Communications Surveys Tutorials 15, 1 (First 2013), 223-239.

[148] WANG, J., WANG, Z., XIA, Y., AND WANG, H. A practical approach for channel assignment in multi-channel multi-radio wireless mesh networks. In Proceedings of the 4th International Conference on Broadband Communications, Networks and Systems, (Raleigh, NC, USA, 2007), BROADNETS'07, IEEE, pp. 317-319.

[149] WANG, X., AND KAR, K. Throughput modelling and fairness issues in CSMA/CA based ad-hoc networks. In Proceedings of the 24th Annual Joint Conference of the Computer and Communications Societies. (Miami, FL, USA, March 2005), INFOCOM'05, IEEE, pp. 23-34 vol. 1.

[150] Wellons, J., AND XUE, Y. The robust joint solution for channel assignment and routing for wireless mesh networks with time partitioning. Ad Hoc Networks, Elsevier 13 (2014), 210-221.

[151] Willig, A., AND UhlemanN, E. On relaying for wireless industrial communications: Is careful placement of relayers strictly necessary? In Proceedings of the 9th International Workshop on Factory Communication Systems (Lemgo, NRW, Germany, May 2012), WFCS'18, IEEE, pp. 191-200.

[152] WU, H., AND PAN, Y. Medium access control in wireless networks, vol. 8. Nova Publishers, 2008.

[153] WU, Z., AND HU, Y. H. How many wireless resources are needed to resolve the hidden terminal problem? . Computer Networks, Elsevier 57, 18 (2013), 3987-3996. 
[154] Xhafa, F., SÁnchez, C., Barolli, A., And TakizaWA, M. Solving mesh router nodes placement problem in wireless mesh networks by Tabu search algorithm. Journal of Computer and System Sciences, Elsevier 81, 8 (2015), 1417-1428.

[155] Xing, K., Cheng, X., MA, L., And LiAnG, Q. Superimposed code based channel assignment in multi-radio multi-channel wireless mesh networks. In Proceedings of the 13th Annual International Conference on Mobile Computing and Networking (Montral, Qubec, Canada, 2007), MobiCom '07, ACM, pp. 15-26.

[156] XU, K., Gerla, M., AND BAE, S. How effective is the IEEE 802.11 RTS / CTS handshake in ad hoc networks? In Proceedings of the Global Telecommunications Conference, (Taipei, Taiwan, Nov 2002), vol. 1 of GLOBECOM'02, IEEE, pp. 72-76 vol.1.

[157] XU, X., SAAD, W., ZHANG, X., XU, X., AND ZHOU, S. Joint deployment of small cells and wireless backhaul links in next-generation networks. IEEE Communications Letters 19, 12 (Dec 2015), 2250-2253.

[158] YANG, J., WANG, Y., HuA, K., AND WANG, W. Fairness based dynamic channel allocation in wireless mesh networks. In Proceedings of the International Conference on Computing, Networking and Communications (Honolulu, HI, USA, 2014), ICNC'14, IEEE, pp. 556-560.

[159] YANG, X., AND VAIDYA, N. On physical carrier sensing in wireless ad hoc networks. In Proceedings of 24th Annual Joint Conference of the IEEE Computer and Communications Societies (Miami,Florida, USA, 2005), INFOCOM'05, IEEE, pp. 2525-2535.

[160] ZENG, Z., YANG, Y., AND HOU, J. C. How physical carrier sense affects system throughput in IEEE 802.11 wireless networks? In proceedings of the 27th Conference on Computer Communications (Phoenix, AZ, USA, 2008), INFOCOM'08, IEEE, pp. 2119-2127. 
[161] ZhaO, H., Garcia-Palacios, E., WAnG, S., Wei, J., And Ma, D. Evaluating the impact of network density, hidden nodes and capture effect for throughput guarantee in multi-hop wireless networks. Ad Hoc Networks, Elsevier 11, 1 (2013), 54-69.

[162] Zhou, G., He, T., Krishnamurthy, S., and Stankovic, J. A. Impact of radio irregularity on wireless sensor networks. In Proceedings of the 2nd International Conference on Mobile Systems, Applications, and Services (New York, NY, USA, 2004), MobiSys '04, ACM, pp. 125138. 